


\section{NIST Technical Note 1548}

\section{Electromagnetic Airframe Penetration Measurements of a Beechcraft Premier IA}

Chriss Grosvenor, Dennis Camell, Galen Koepke. and David Novotny

Electromagnetics Division

Electromagnetics and Electrical Engineering Laboratory

National Institute of Standards and Technology

Boulder, CO 80305-3328

Robert T. Johnk

National Telecommunication and Information Agency

Institute for Telecommunications Sciences

Boulder, CO 80305-3328

August 2008

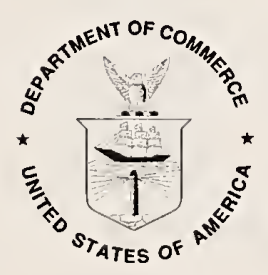

U.S. Department of Commerce

Carlos M. Gutierrez, Secretary

National Institute of Standards and Technology

James M. Turner, Deputy Director 
Certain commercial entities, equipment, or materials may be identified in this document in order to describe an experimental procedure or concept adequately. Such identification is not intended to imply recommendation or endorsement by the National Institute of Standards and Technology, nor is it intended to imply that the entities, materials, or equipment are necessarily the best available for the purpose.

National Institute of Standards and Technology

Technical Note 1548

Natl. Inst. Stand. Technol.

Tech. Note 1548

110 pages (August 2008)

CODEN: NTNOEF
U.S. Government Printing Office Washington: 2008
For Sale by the Superintendent of Documents U.S. Government Printing Office Stop SSOP, Washington, DC 20402-0001 Phone: (202) 512-1800 Fax: (202) 512-2250 Internet: bookstore.gpo.gov 


\section{Contents}

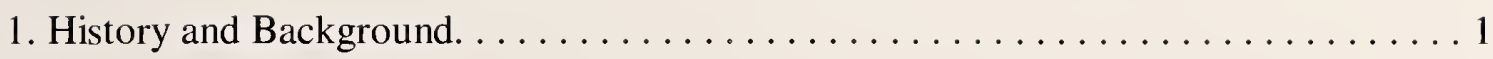

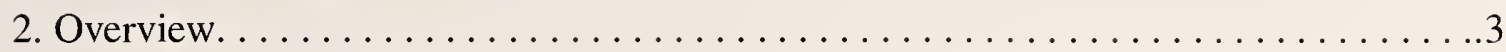

3. Measurement System ............................... 5

4. Measurement of Electromagnetic Airframe Penetration ................ 7

5. Reference Measurements . ............................. 10

6. Airframe Penetration Measurements - Overview. . . . . . . . . . . . . . . . 15

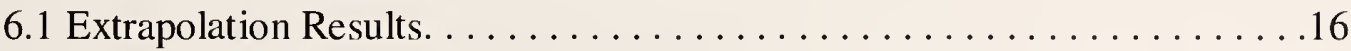

6.2 Main Passenger Cabin Penetration Results. ............... 18

6.3 Flight Deck Penetration Results. . . . . . . . . . . . . . . . . . 40

6.4 Rear Cargo Hatch Penetration Results. . . . . . . . . . . . . . .55

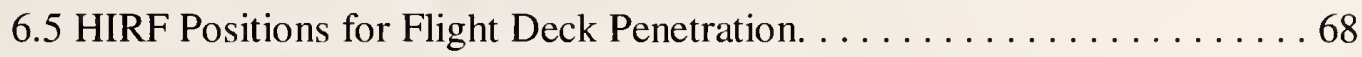

6.6 Internal Coupling Measurements. . . . . . . . . . . . . . 73

7. Uncertainty Analysis. . . . . . . . . . . . . . . . . . . . . 81

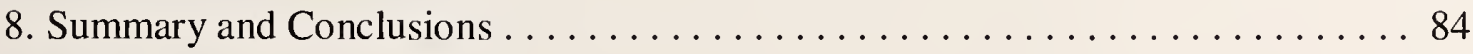

9. References. . . . . . . . . . . . . . . . . . . . . . . . . . 87

Appendix A. Time-Domain Waveform Analysis. . . . . . . . . . . . . . 89

Appendix B. Signal and Noise Characteristics ................. 93

Appendix C. TEM and DRG Horn Boresight Gain Characteristics. ........95

Appendix D. Carbon Fiber Composites. . . . . . . . . . . . . . . .97

D.1 Dielectric Properties . . . . . . . . . . . . . . . . . 98

D.2 Shielding Effectiveness .................... 99

Appendix E. Penetration Data for HIRF Frequency Bands . . . . . . . . . . 101

Appendix F. Beechcraft Premier IA Measurement Equipment List . . . . . . 105 



\title{
Electromagnetic Airframe Penetration Measurements of a Beechcraft Premier IA
}

\author{
Chriss Grosvenor, Dennis Camell, Galen Koepke, and David Novotny \\ Electromagnetics Division \\ National Institute of Standards and Technology \\ 325 Broadway \\ Boulder, CO 80305 \\ and \\ Robert T. Johnk \\ National Telecommunication and Information Agency \\ Institute for Telecommunication Sciences \\ 325 Broadway \\ Boulder, CO 80305
}

The National Institute of Standards and Technology has recently completed shielding effectiveness/penetration studies on three different aircraft types for the Federal Aviation Administration. The studies are used to understand the cavity coupling characteristics between antennas placed in various compartments inside the aircraft and antennas placed at various angular positions around an aircraft. This document will show how penetration varies as a function of frequency, antenna type, antenna polarization, and cavity susceptibility. Internal coupling between two antennas placed in the aircraft at different locations to determine the quality factor and the time decay of fields is also presented. This technical note gives results for the Beechcraft Premier IA.

Key words: aircraft; cavity coupling; digital signal processing; HIRF; penetration; shielding effectiveness; synthetic time-domain measurements.

\section{History and Background}

The Field Parameters and EMC Applications project team of the National Institute of Standards and Technology (NIST) has recently completed shielding effectiveness studies of three representative aircraft for the Federal Aviation Administration (FAA). These studies will be used to provide the FAA with the procedures and data reduction techniques for typical low-level airframe high intensity radiated fields (HIRF) attenuation/shielding tests. This technical note addresses aircraft shielding tests conducted on a Beechcraft Premier IA carbon-fiber composite business jet. The first study was a technical note reporting the results from the FAA's Bombardier Global 5000 business jet [1], and the final report will be on a Boeing 737-200, owned and operated by the FAA. The Bombardier Global 5000 represents a typical business jet and the 737-200 represents a typical commercial aircraft. By measuring all three aircraft and 
comparing the results we will have the potential to provide a "design roadmap" for the optimization of HIRF testing standards for the EMC aircraft manufacturing community.

The FAA currently has specific certification policies that address HIRF testing on an aircraft's electrical and electronic systems. These certification tests have been applied for the past 19 years [2]. In the FAA document, the regulatory authorities and industry have defined HIRF environments, requirements for aircraft HIRF protection, and methods for testing and verifying the level of HIRF protection. Most of reference [2] discusses HIRF testing for electronic and electrical subsystems, which this technical note does not address. This technical note specifically addresses both high- and low-level airframe shielding compliance testing. The HIRF procedures are found in sections 6.6.2.6 and section 6.6.4 of reference [2].

The basic problem is to evaluate the coupling of an external source into a large, leaky cavity [36]. The cavity could represent the whole aircraft or a cavity (cargo bay, flight deck, etc.), depending on the expected vulnerability. In a typical HIRF measurement, the measurement system is calibrated and a reference measurement is taken without the aircraft embedded in the test setup. The aircraft is then placed into the test setup and various measurements are taken in susceptible cavities. These attenuation tests are typically conducted using either mechanical stirring or frequency stirring methods [1-3]. The airframe attenuation is calculated by use of the following equation,

$$
A_{\text {atten }}=S . E .=\frac{\text { reference measurement }}{\text { cavity measurement }},
$$

where $\mathrm{A}_{\text {atten }}$ is the airframe attenuation and S.E. is defined as the shielding effectiveness. These numbers, expressed in decibels, are typically positive so that the more positive the number, the greater the protection the aircraft provides to electromagnetic fields, and as the number approaches zero we expect almost no shielding protection. The aircraft HIRF attenuation data vary as a function of frequency, physical position of the measurement antenna, characteristics of the aircraft, and configuration of the test site. After a company tests and processes the data, the information is given to the FAA, which uses it, in conjunction with the subsystem susceptibility tests, to determine an aircraft's HIRF vulnerability.

The detailed method used to process aircraft HIRF attenuation data is not well documented and is closely held by the handful of test service providers. Therefore, the interpretation of the data is not immediately obvious to the certification engineer. This technical note, the Bombardier technical note [1], and the forthcoming Boeing 737-200 technical note, will consider attenuation measurements as a function of frequency, antenna position, antenna type, and cavity selection. Our data-processing methodology will be shown in detail. Using this information, we hope to provide guidelines for both the FAA and test engineers to develop a uniform and transparent test method for data processing and documentation. We know that the confidence levels are expected to be dependent on the number of sample points (measurement geometries) but we 
should be able to assess the trade-offs between measurement effort (sample number) and risk (confidence level).

\section{Overview}

This report summarizes results of a measurement effort conducted by the Field Parameters and EMC Applications Project team of NIST. This effort consisted of an extensive series of penetration measurements performed on a Beechcraft Premier IA designed and owned by the Hawker Beechcraft Corporation. The aircraft was tested at the Hawker Beechcraft facility in Wichita, Kansas during the period from April 16, 2007 to April 20, 2007. The purpose of this effort is to develop a test methodology that allows us to understand the differences between various types of aircraft construction.

The effort utilized a NIST-developed measurement system consisting of ultra-wideband transverse electromagnetic (TEM) horn antennas, 3117 dual-ridged guided (DRG) horn antennas, a commercially available vector network analyzer (VNA), and interconnecting transmission media, and an amplifier. Interconnections between transmitting and receiving antennas were affected using broadband $11 \mathrm{GHz}$ and $18 \mathrm{GHz}$ precision, analog, electro-optic links in conjunction with a configurable VNA and amplifier for airframe shielding studies up to $18 \mathrm{GHz}$. Shielding data were obtained from a direct comparison of two measurements: (1) transmission measurements between boresighted antennas located outside the aircraft to obtain a reference, and (2) transmission measurements with one antenna located inside a selected compartment of the aircraft and a pair of horizontally and vertically polarized antennas for both low-frequencyband and high-frequency-band antennas located outside the aircraft at specified locations. Shielding data were obtained by comparing processed reference and aircraft transmission data. This is achieved using an efficient sequence of Fourier and inverse Fourier transformations, frequency domain convolutions, and filtering combined with time gating and frequency averaging. Shielding values were obtained by taking the ratio of the gated amplitude spectra of the aircraft measurement and the gated amplitude spectra of the references. Data smoothing is performed using the frequency-averaging of signal power over a specified bandwidth as described in reference [3],

$$
\left\langle S E\left(f_{n}\right)\right\rangle=\frac{1}{2 N+1} \sum_{i=n-N}^{n+N}\left|S E\left(f_{i}\right)\right|^{2} .
$$

Shielding data were obtained with a receiving antenna located in one of three internal compartments: (1) the main passenger cabin, (2) the flight deck, and (3) the external, rear cargo hatch. A pair of transmitting antennas were positioned outside the aircraft at a fixed height of $3 \mathrm{~m}$, which is the approximate height of the windows, at a fixed distance of $23 \mathrm{~m}$ from the center of the aircraft. The transmitting antennas were boresighted at the center of the aircraft, and data were obtained for both horizontal and vertical polarizations. We based our tests on HIRF standard test procedures and previous experience with aircraft shielding measurements. These standards are concerned with detecting possible leakage into the aircraft. Key areas include the flight deck, the avionics bay, any windows/doors/joints, and the wing/tail section of the fuselage. 
We defined a test setup at various angular positions around the aircraft, around the flight deck of the aircraft at five positions at a constant distance from the front landing gear of the aircraft, and internal coupling measurements. In a recent paper [4], questions were raised as to how many positions need to be measured around an electrically large object to fully characterize the fields penetrating into the aircraft for a particular high-frequency limit. For this reason, we designed the test plan as follows: For orbit 1 , receiving antennas were placed in the main passenger cabin and (1) measurements were taken from $0^{\circ}$ to $120^{\circ}$ every $10^{\circ}$; (2) measurements were taken every $5^{\circ}$ from $120^{\circ}$ to $150^{\circ}$; and (3) measurements were taken at $160^{\circ}, 170^{\circ}, 180^{\circ}, 190^{\circ}, 270^{\circ}$, and $330^{\circ}$. For orbits 2 and 3 , receiving antennas were placed in the flight deck and in the rear cargo hatch, and measurements were taken every $10^{\circ}$, which was shown to be sufficient from the first orbit and were taken around the entire aircraft. By traversing around the aircraft we could look at the symmetry behavior of the carbon-fiber composite structure. After these three orbits, we concluded with a set of internal coupling measurements to understand the reverberant environment internal to the aircraft. An aircraft's level of protection, referred to as shielding, depends on the level of treatment, the compartment, the frequency, the transmitting antenna polarization, and the angle of illumination.

In this document, we will display penetration values, which are the reciprocal of shielding values. On the graphs that follow, a penetration near $0 \mathrm{~dB}$ means electromagnetic fields can easily penetrate into that part of the aircraft. Penetration levels for the Premier IA range from approximately $0 \mathrm{~dB}$ to $-40 \mathrm{~dB}$. The estimated dynamic range of the measurement system is 50 $\mathrm{dB}$ at $4000 \mathrm{MHz}$ and $30 \mathrm{~dB}$ at $18,000 \mathrm{MHz}$. Maximum penetration was observed with the receiving antenna in the main passenger cabin, while lower values were obtained in the flight deck and the rear cargo hatch. Penetration in the main passenger cabin was fairly consistent around the aircraft at values between $-10 \mathrm{~dB}$ and $-15 \mathrm{~dB}$ across the frequency band. Minimum shielding in the main passenger cabin was observed around the tail of the aircraft at a value of around $-20 \mathrm{~dB}$ for the antennas in the vertical polarization. Minimum penetration, for the aircraft, was seen in the rear cargo hatch at positions between $10^{\circ}$ and $90^{\circ}$.

This report is divided into five main sections: (1) a description of the measurement system, (2) an overview of the measurement technique, (3) an overview of the signal processing, (4) a summary of results obtained for the three different aircraft compartments and various internal measurements, and (5) an uncertainty analysis. Six appendices discuss the impact of time gating, signal and noise, an equipment list, maximum penetration as a function of the HIRF specified frequency bands, the gain characteristics of the ultra wideband antennas that were used, and a discussion of the physical properties, fabrication, and electrical properties of carbon-fiber composite materials. 


\section{Measurement System}

The configuration of the NIST measurement system for these aircraft measurements is shown in Figures 1 and 2. The system consists of: (1) a VNA, (2) two transmitting TEM horn antennas and two DRG horn antennas configured for horizontal and vertical polarizations, respectively, (3) a receiving TEM horn antenna and DRG antenna, (4) two coaxial microwave switches to select the polarization of the transmit antennas, and (5) two precision analog electro-optic links to cover both frequency bands and to provide an interconnection between the transmitting antennas outside the aircraft and the receiving antenna inside. These links provide a significant improvement in performance over conventional microwave cables. The optical links result in low transmission losses, low noise, and they do not pick up common-mode noise from environmental ambient signals. The optical links also provide an improvement in dynamic range and have better immunity from electromagnetic interference.

The heart of this system is a commercially available four-port VNA that has been configured to acquire complex transmission data $\left(S_{31}\right.$ for the TEM antennas and $S_{42}$ for the DRG antennas, inphase and quadrature signals). Due to internal hardware limitations, the VNA was limited to a maximum of 16001 frequencies, but we developed software that extends the number of frequencies to any desired value. If we need more than 16001 points, the software subdivides a given frequency range into a user-selected number of 16001 point sub-bands. The DRG horn antennas operate from $1 \mathrm{GHz}$ to $18 \mathrm{GHz}$ and were configured using two bands resulting in 32002

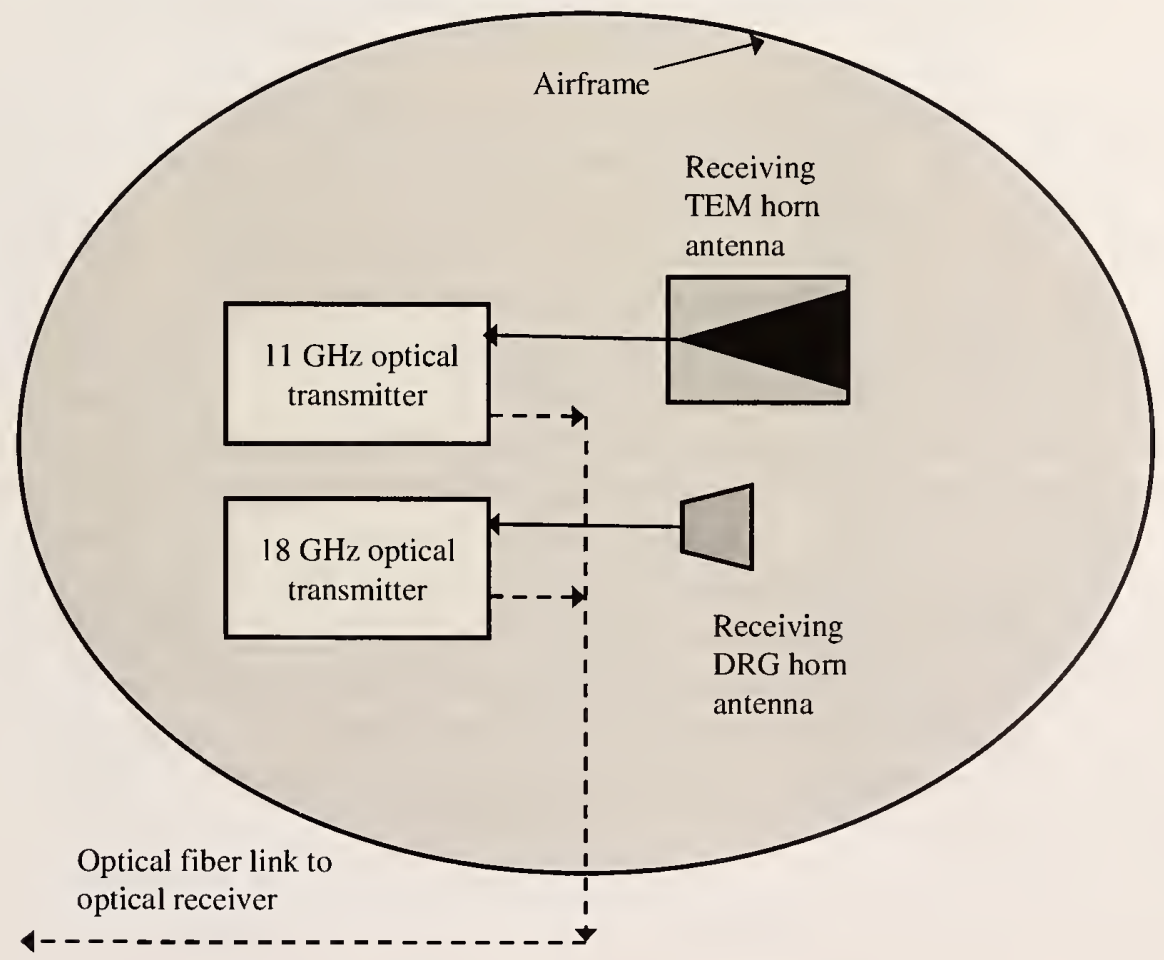

Figure 1. Airframe shielding measurement system (receiving side). The reverberant nature of the airframe permits the reception of horizontal and vertical polarized signals. 


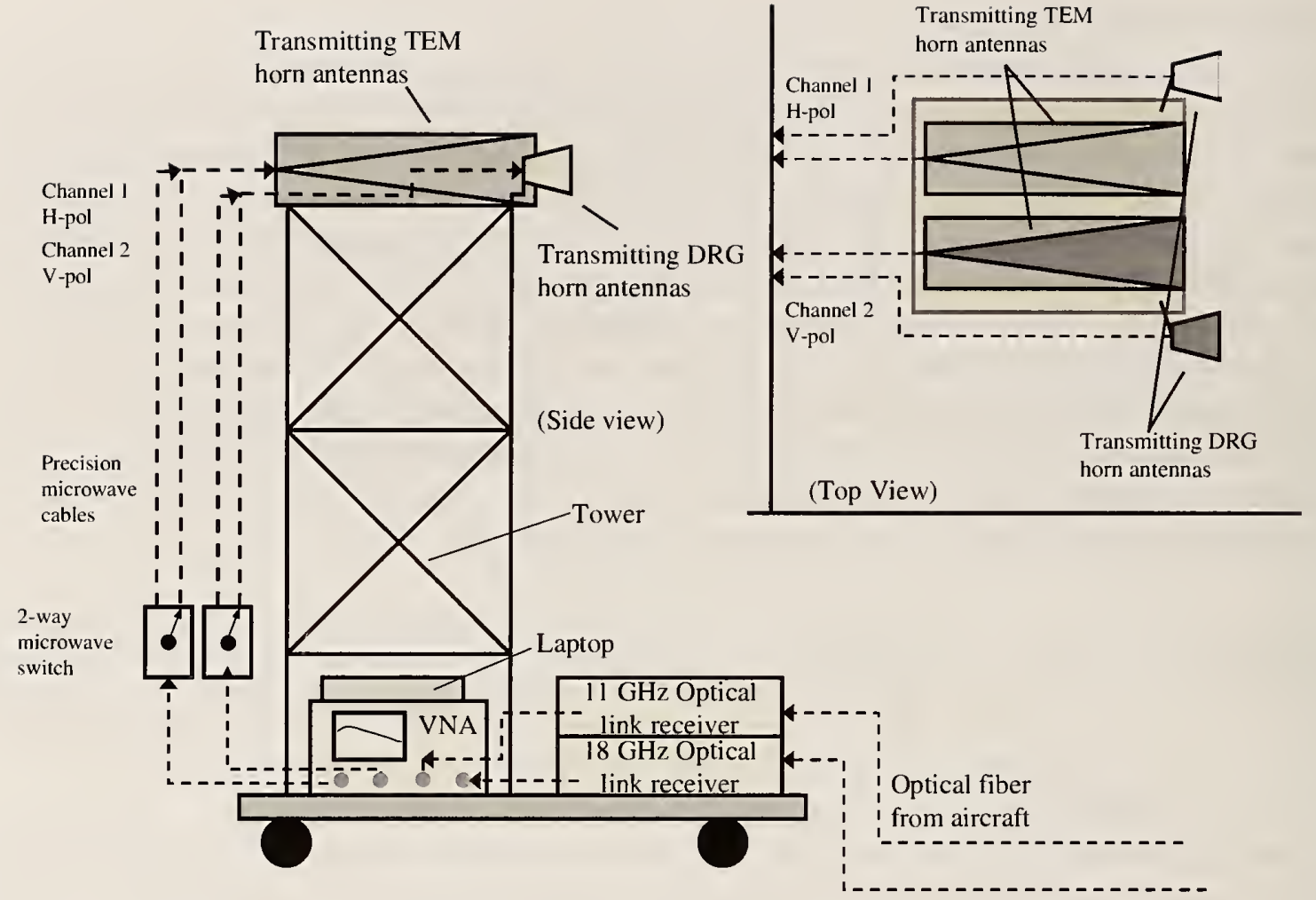

Figure 2. Transmitting side of the airframe shielding measurement system.

equally spaced measurement points from approximately $0.5 \mathrm{MHz}$ to $18 \mathrm{GHz}$. The TEM horn antennas operate from $0.1 \mathrm{GHz}$ to $4 \mathrm{GHz}$ and were configured using one band, resulting in 6401 equally spaced measurement points from approximately $0.5 \mathrm{MHz}$ to $4 \mathrm{GHz}$. A reduced frequency spacing is necessary to avoid problems due to aliasing. Prior to connecting the cables to the antennas, a calibration of all channels was performed to remove systematic transmission effects due to cabling, switches, and the frequency and phase variations of the optical transmission link. This procedure calibrates the system to the input of the transmitting and receiving antennas. Three NIST-developed ultra-wideband TEM horn antennas were used: two $1.2 \mathrm{~m}$ antennas on a tower located outside of the aircraft, either to transmit horizontally and vertically polarized fields, and a more compact $36 \mathrm{~cm}$ TEM horn located in a preselected aircraft compartment to detect energy coupled into the airframe. For the higher frequency bands three commercially available DRG antennas were used: two on the same transmitting tower located outside of the aircraft to transmit horizontally and vertically polarized fields, and a DRG antenna located in the same preselected aircraft compartment to detect energy coupled into the airframe. The frequency data were digitized and transferred to a laptop computer for subsequent data analysis and signal processing. 


\section{Measurement of Electromagnetic Airframe Penetration}

The extraction of airframe penetration characteristics required the two-step measurement process, shown in Figure 3. In the first step, a reference transmission measurement was performed by boresighting the transmitting and receiving antennas at a fixed distance. The reference measurements were performed at a location away from the aircraft in order to minimize reflections from the aircraft. This measurement quantifies the energy incident on the aircraft, and enables us to calibrate and remove the frequency-dependent effects of the antennas and interconnecting hardware. In the second step, the receiving antenna was placed in a selected aircraft compartment, the transmitting antennas were placed at fixed locations, external to the aircraft, and stepped-frequency transmission data were acquired.

The reference and airframe data were processed using a sequence of filtering, Fourier transforms, and time gating to obtain the penetration values. The basic process through which the data were obtained is shown in Figure 4. The raw frequency data were first filtered to maximize signal-tonoise ratio and to minimize effects from the low-frequency cut-off of our electro-optic link. Next, a tapered frequency-domain window was applied to both sets of data to reduce Gibbs ringing in subsequent processing. An inverse Fourier transform (IFT) was then applied to the processed data to obtain time-domain waveforms. Time gates were then applied to isolate the desired portions of waveforms and remove undesired portions. In the case of the reference, the time gate was applied to isolate the direct antenna-to-antenna coupling and remove the effects of
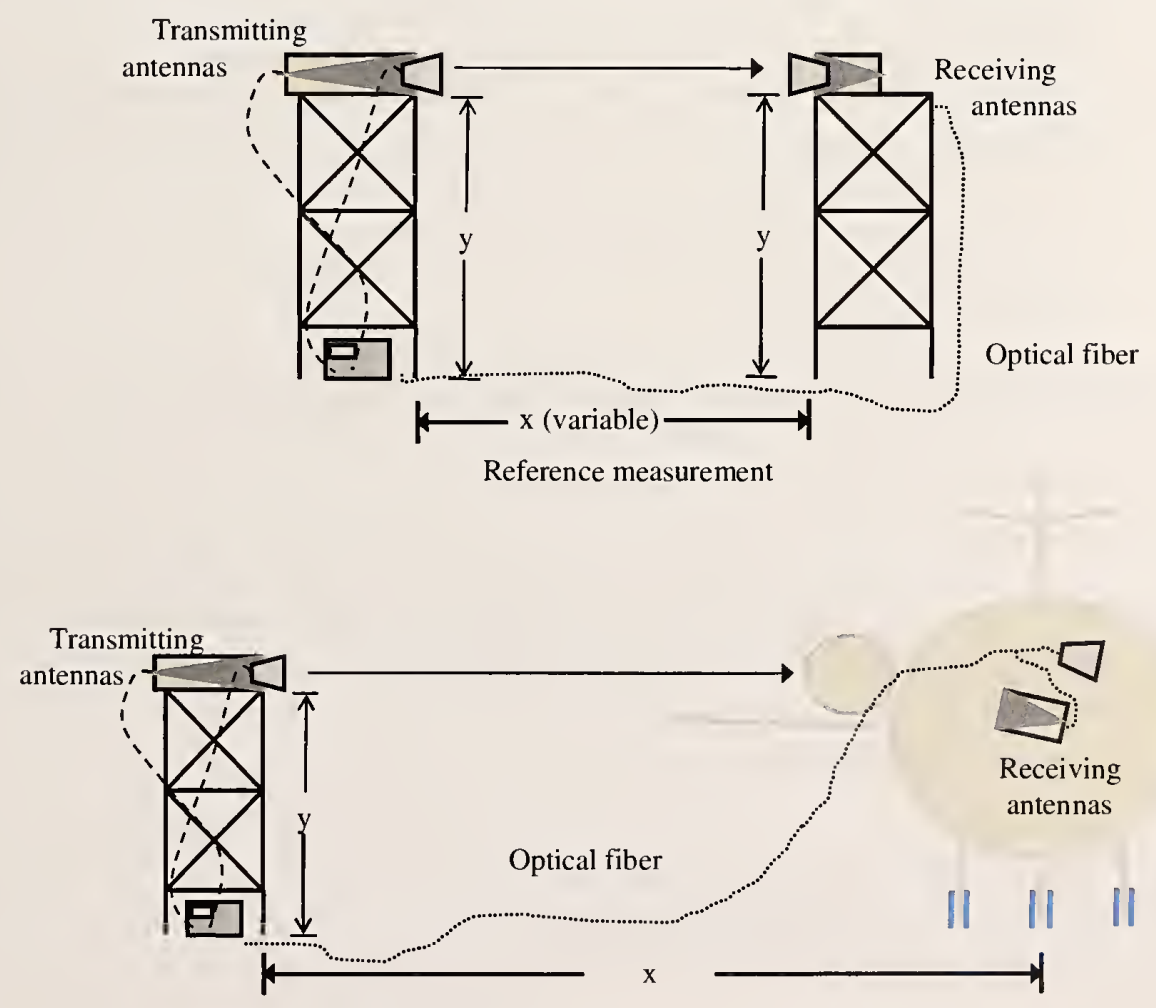

Figure 3. Two-step airframe shielding measurement procedure used in the Beechcraft Premier IA evaluation. 
Transmission measurement through airframe

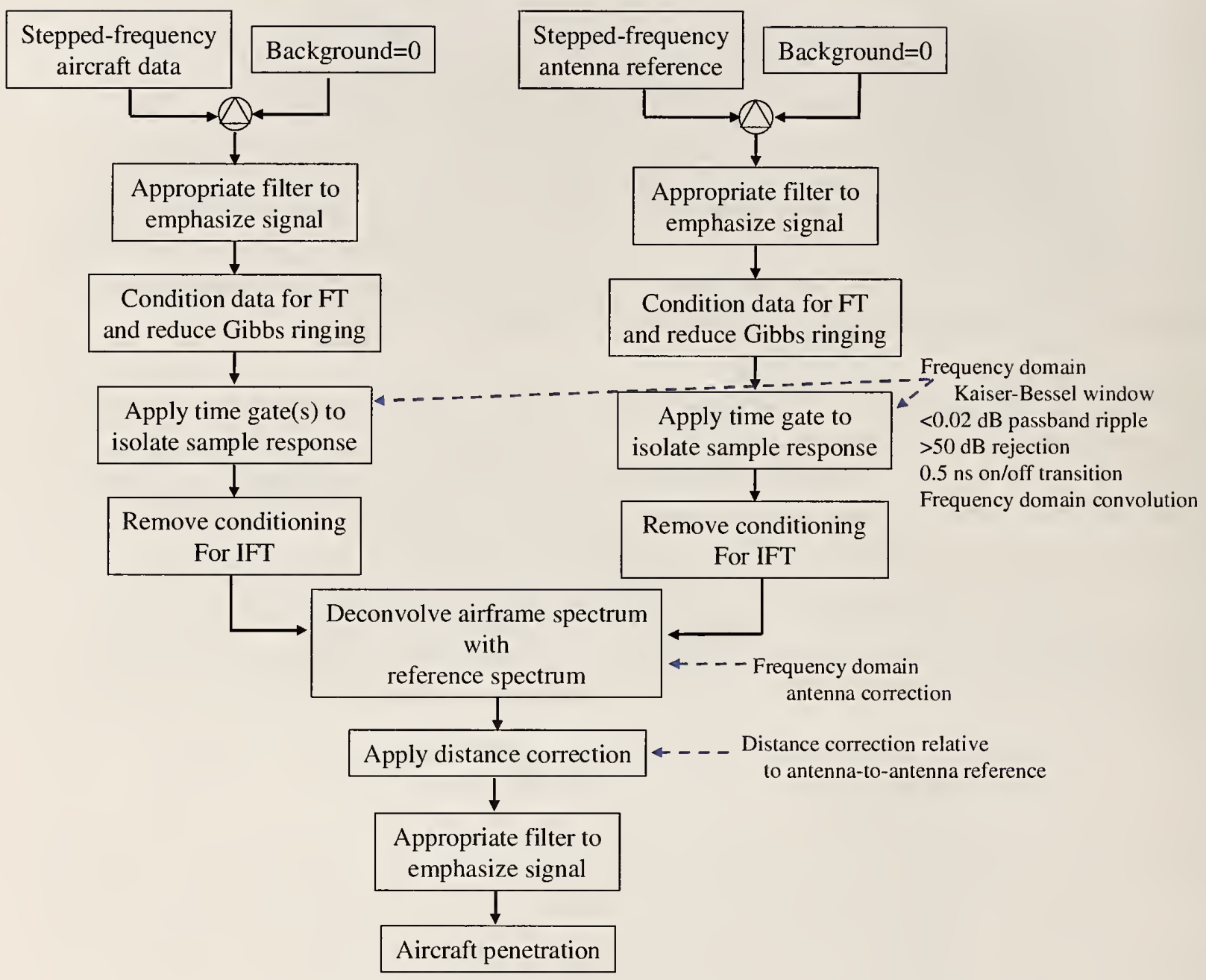

Figure 4. Signal processing sequence to obtain airframe penetration.

ground bounce and late-time reflections. Time gating was applied to the airframe data to isolate either the direct illumination or reverberant fields of the aircraft. Effects from ground bounce were embedded in the waveforms at this distance and therefore cannot be removed by timegating. The time-gated data sets were then transformed back into the frequency domain, and the penetration $(P E)$, was computed from the deconvolution:

$$
P E=D C \cdot\left|\frac{S_{42}(\text { gated } \text { airframe })}{S_{42}(\text { gated } \text { reference })}\right|^{2},
$$

where $S_{42}$ (gated reference) is the antenna-to-antenna coupling with environmental effects gated out, and $S_{42}$ (gated airframe) is the airframe transmission with gating applied to minimize noise. 


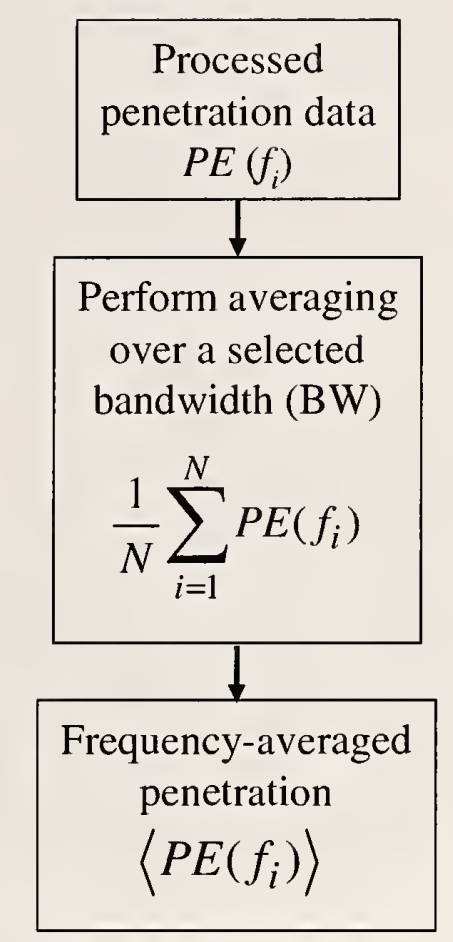

Figure 5. The frequency averaging definition.

Since the reference and airframe measurements were typically carried out at different distances, a distance correction $(D C)$ was applied:

$$
D C(d B)=20 \log _{10}\left(\frac{D}{D_{r e f}}\right),
$$

where $D$ is the distance in meters from the aperture of the transmitting antenna to the geometric center of the aircraft and $D_{\text {ref }}$ is the distance between the reference antennas. A detailed description of this procedure is given in reference [3]. Thus, our definition of penetration is based on a comparison of a direct antenna-to-antenna coupling and signal transmission through the airframe. The penetration, as we define it here, is the reciprocal of shielding (in decibels, penetration is the negative of shielding). We prefer to display results in terms of penetration because of a better graphical display and so that we normalize by our reference measurement.

Single aspect penetration characteristics for aircraft exhibit rapid variations with frequency, due to the large, complex cavity behavior of the aircraft. In order to obtain the volumetric averaged penetration for a compartment, the processed penetration data are frequency averaged over a specified bandwidth, by use of the process shown in Figure 5. Averaging the penetration data reduces the complex cavity variations and highlights systematic coupling effects. These effects are discussed in detail in references [1-3]. 


\section{Reference Measurements}

The test setup for the reference measurements is shown in Figure 6. The transmitting antennas are shown mounted on one tower with aperture centers located $3 \mathrm{~m}$ above the ground. The receiving antennas were mounted on the other tower. The towers were constructed from PVC pipes and plywood platforms to produce a low-density support structure with minimal scattering. The antennas were then boresighted and a distance extrapolation was performed to determine an optimal reference distance to minimize near-field effects and maximize the period between the signal and ground bounce. We performed multiple reference measurements with antenna-toantenna separations ranging from $2 \mathrm{~m}$ to $35 \mathrm{~m}$. We found that a separation of $6 \mathrm{~m}$ was optimal in terms of accuracy and time separation. Two transmission measurements were performed, namely, one each for the vertical and horizontal channels. This process required a manual reorientation of the receiving antenna to be co-polarized with each of the transmitting antennas. Once the reference data were taken, the receiving antenna was dismounted from the support tower and placed at selected locations inside the aircraft. The external antenna tower and aircraft are shown in Figure 7. The reference waveforms for the DRG antenna are shown in Figures 8, 9, and 10. The reference waveforms for the TEM horn antenna are shown in Figures 11, 12, and 13. The ungated time-domain reference waveform for the DRG antenna is shown in Figures 8 and 11. The doublet that occurs at $15 \mathrm{~ns}$ in Figure 8 and $25 \mathrm{~ns}$ in Figure 11 correspond to the direct antenna-to-antenna coupling. The subsequent waveform activity is due to a combination of internal antenna reflections and environmental scattering due to ground bounce and reflections from nearby objects. Figures 9 and 12 show the ungated frequency-domain response for the DRG and TEM horn references, respectively. The antenna-to-antenna coupling response occurs at $25 \mathrm{~ns}$ for these antennas due to the offset of the apertures. The noise-like hash on the waveform is due to ground bounce and spurious reflections, which were time-gated out of the reference measurement. After isolating the antenna response for both antennas, we obtained the gated frequency-domain response of the DRG and TEM horn antennas in Figures 10 and 13, respectively. These are the waveforms we use as our reference to determine penetration. 


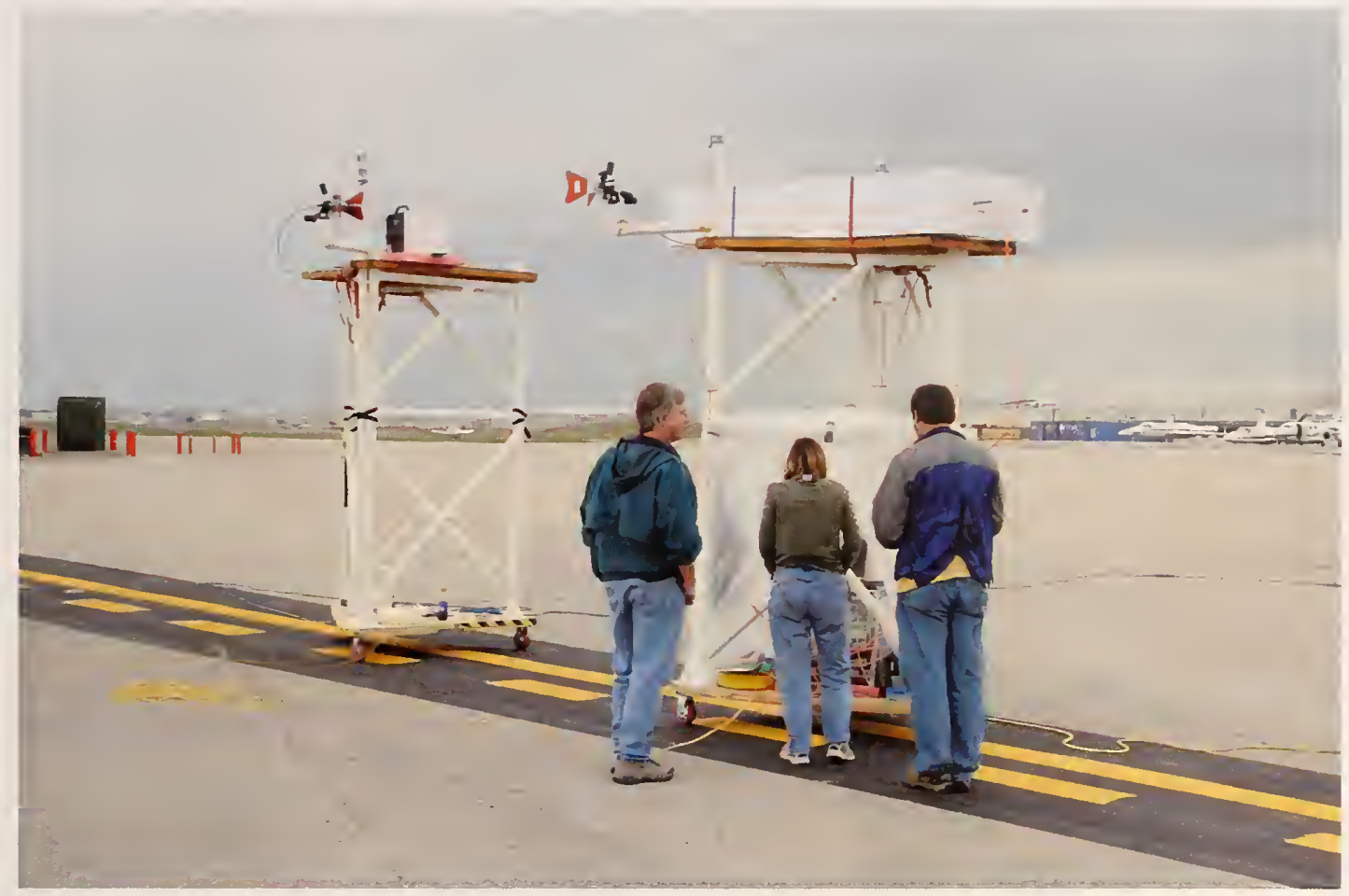

Figure 6. Reference measurement setup. The transmitting tower is configured and the receiving antennas will be mounted on top of the second tower.

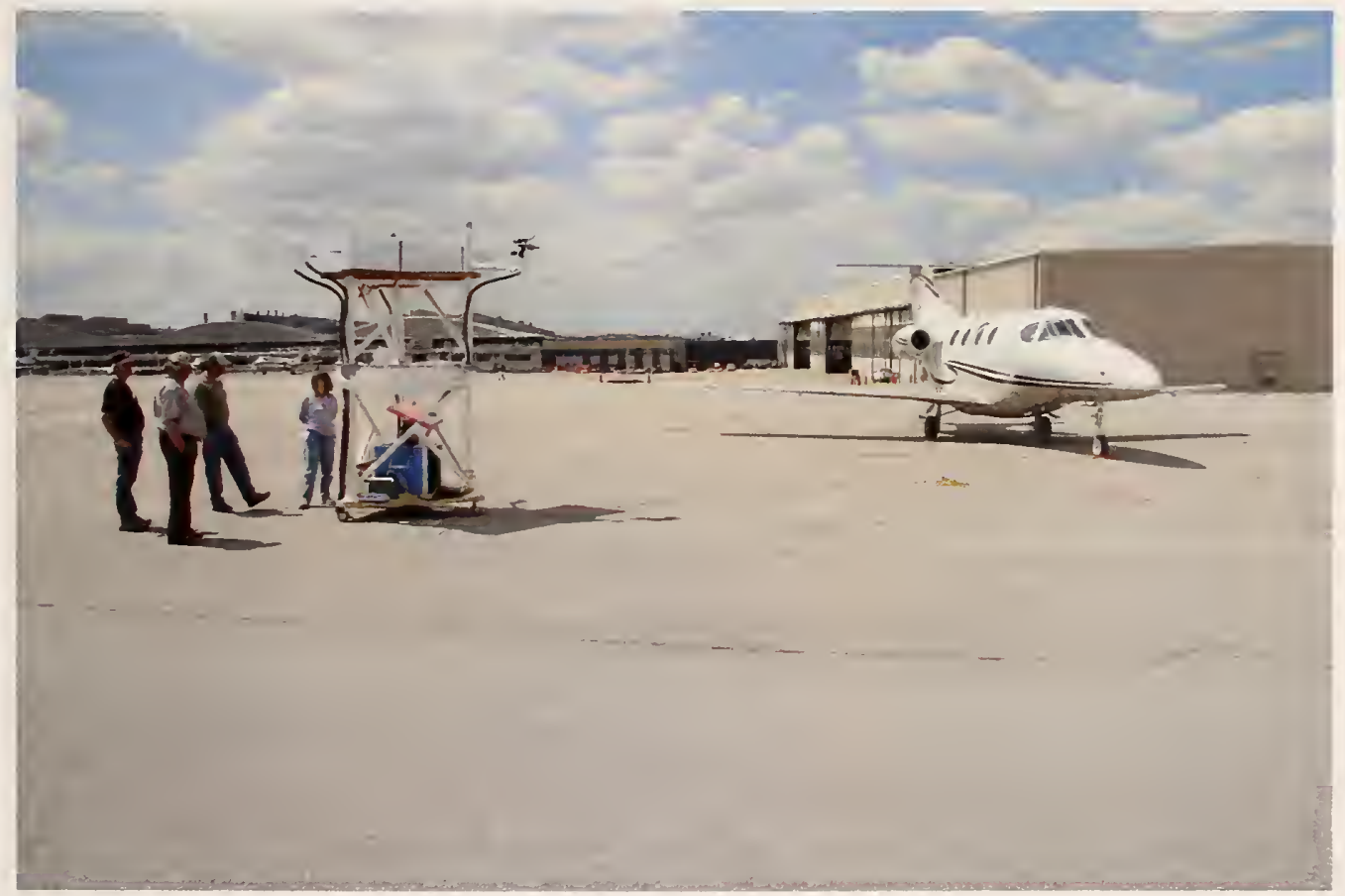

Figure 7. The transmitting antennas placed on top of a support tower and boresighted at the center of the aircraft. 


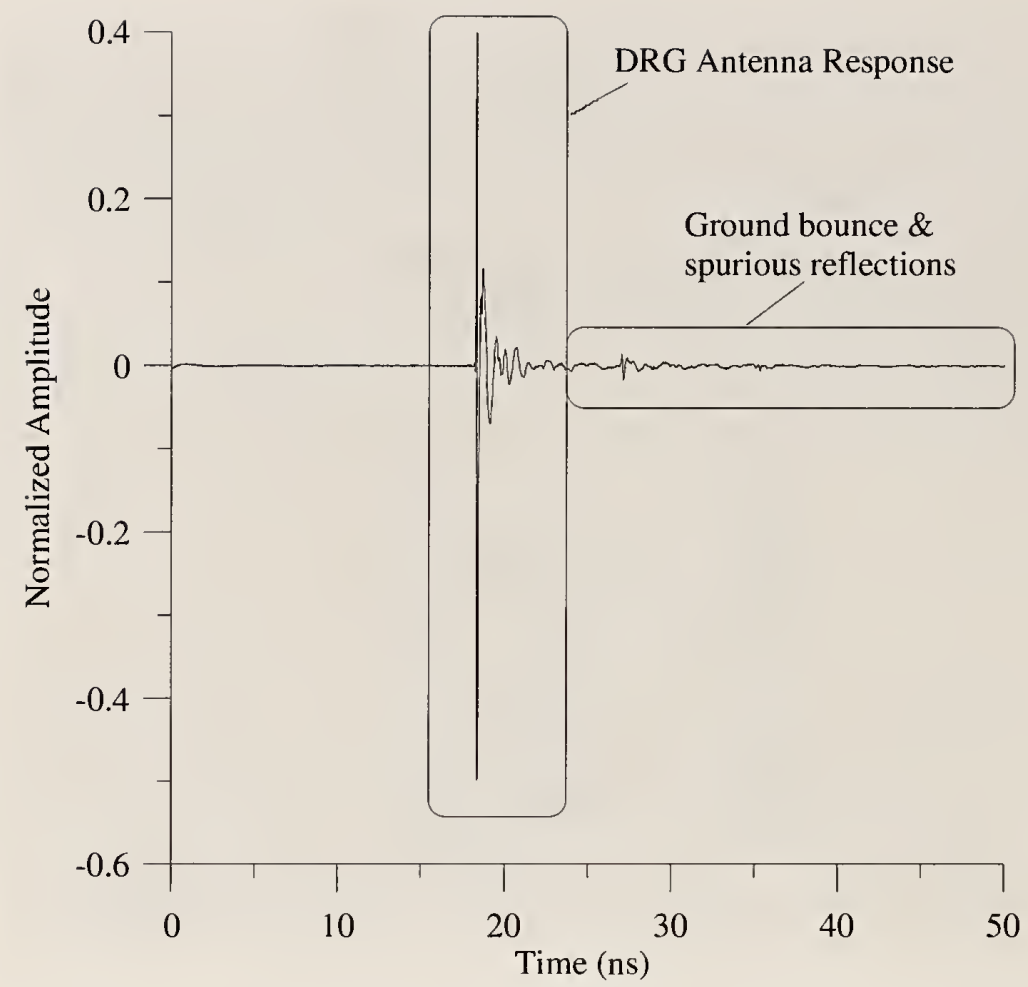

Figure 8. Ungated time-domain transmission waveform for a DRG antenna obtained at a separation of $6.0 \mathrm{~m}$ and vertical polarization.

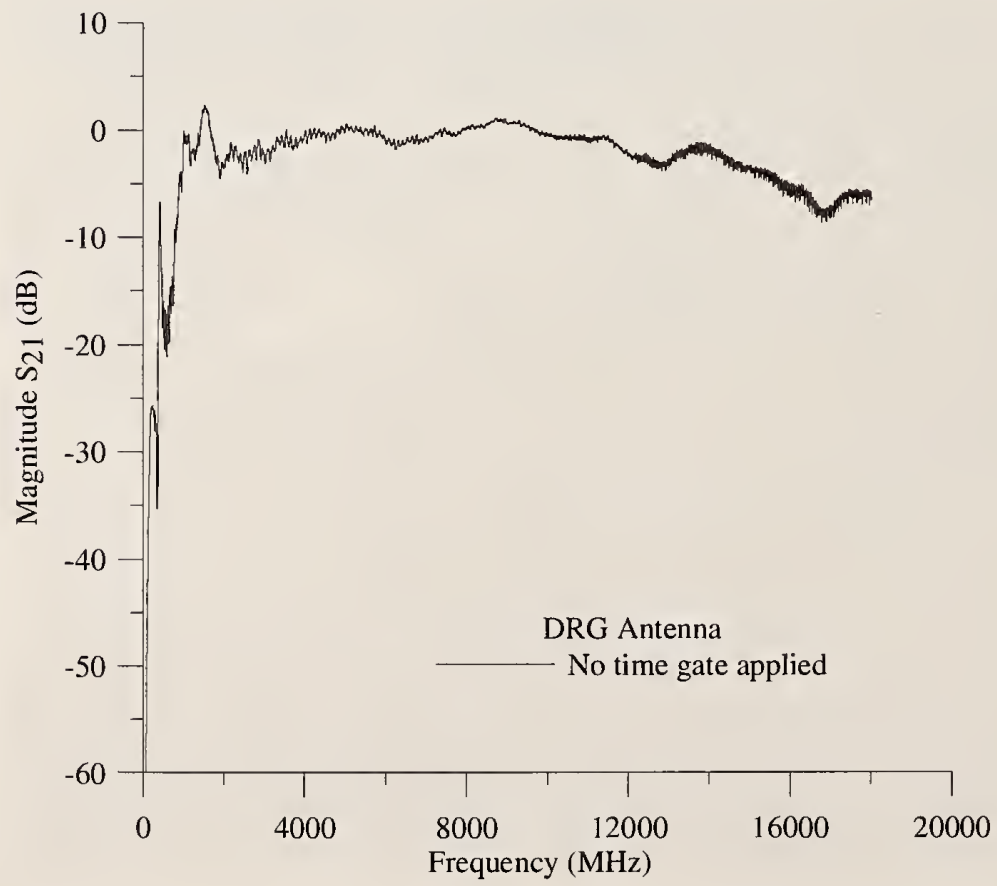

Figure 9. Ungated frequency-domain amplitude spectrum for a DRG horn antenna at a separation of $6 \mathrm{~m}$ and vertical polarization. Note the hashy behavior due to the spurious reflections shown in Figure 8. 


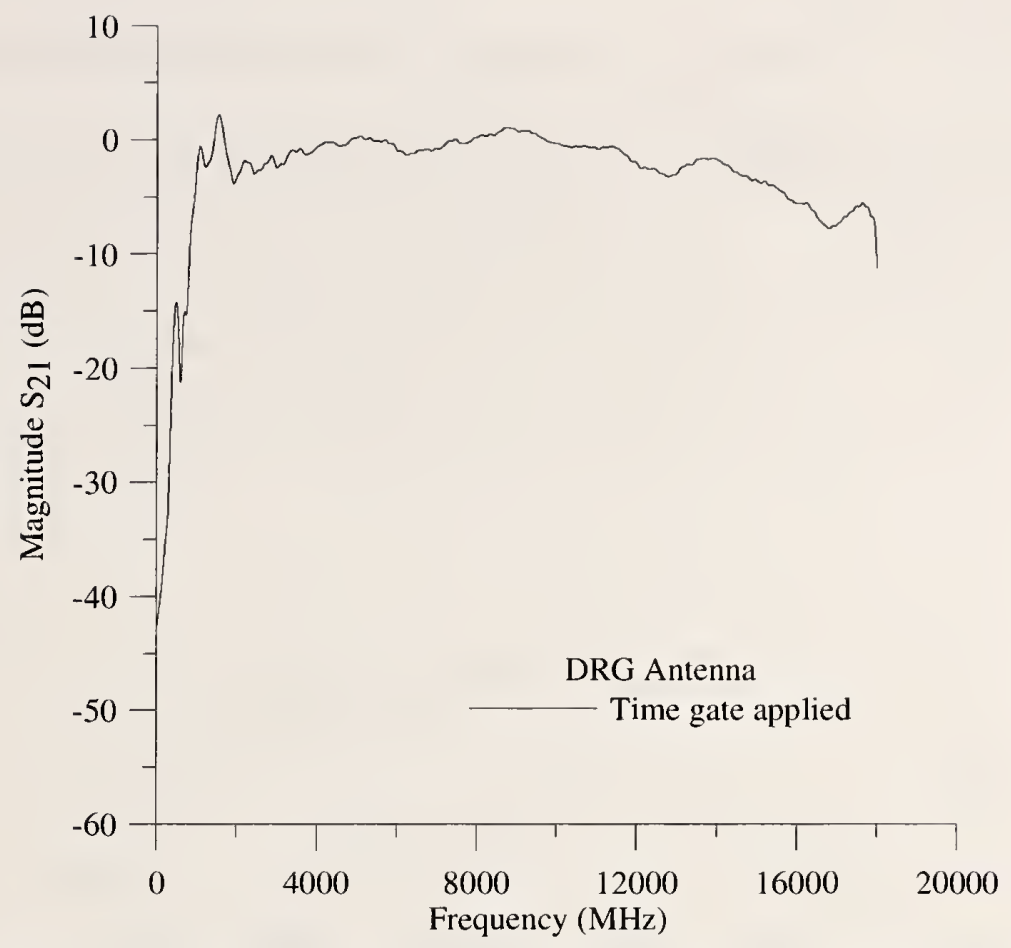

Figure 10. Gated frequency-domain amplitude spectrum corresponding to the waveform of Figure 9.

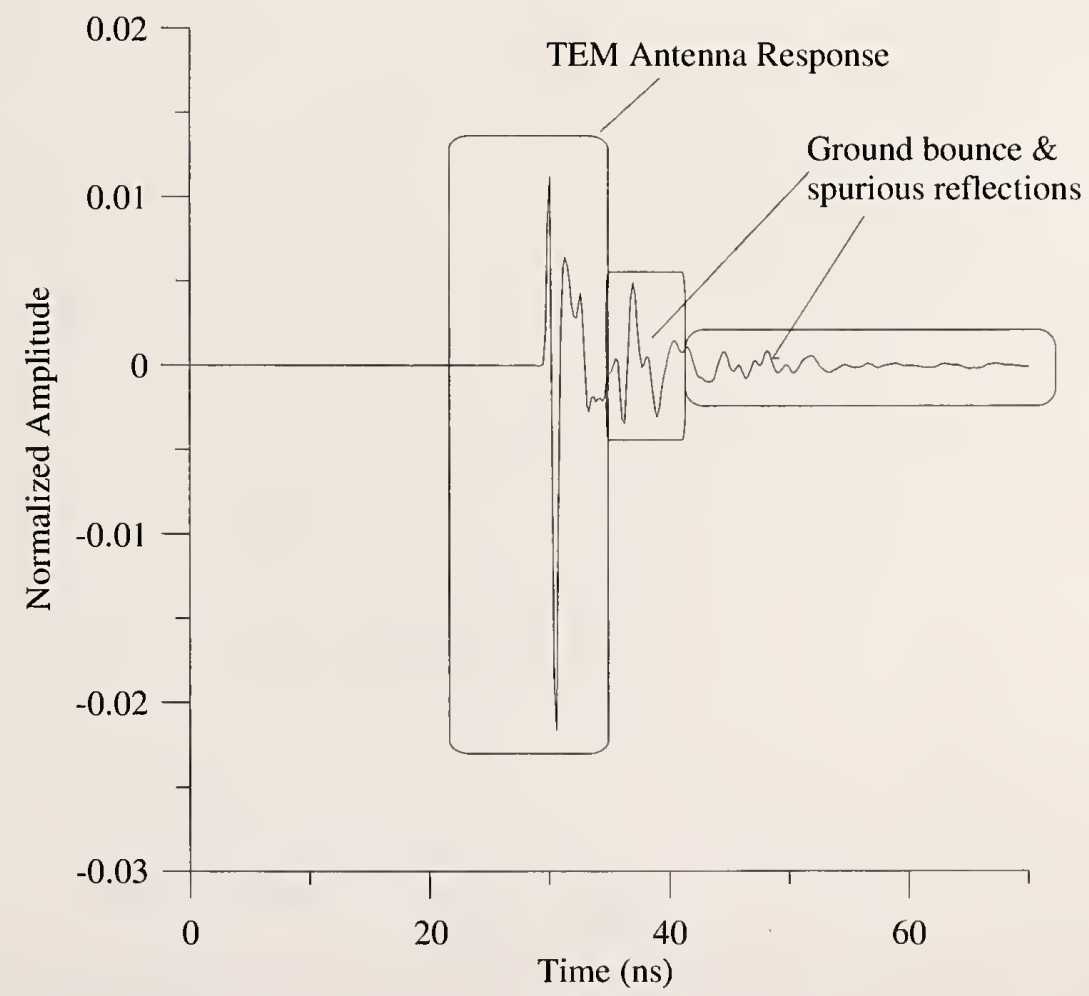

Figure 11. Ungated time-domain transmission waveform for a TEM horn antenna obtained at a separation of $6 \mathrm{~m}$ and vertical polarization. 


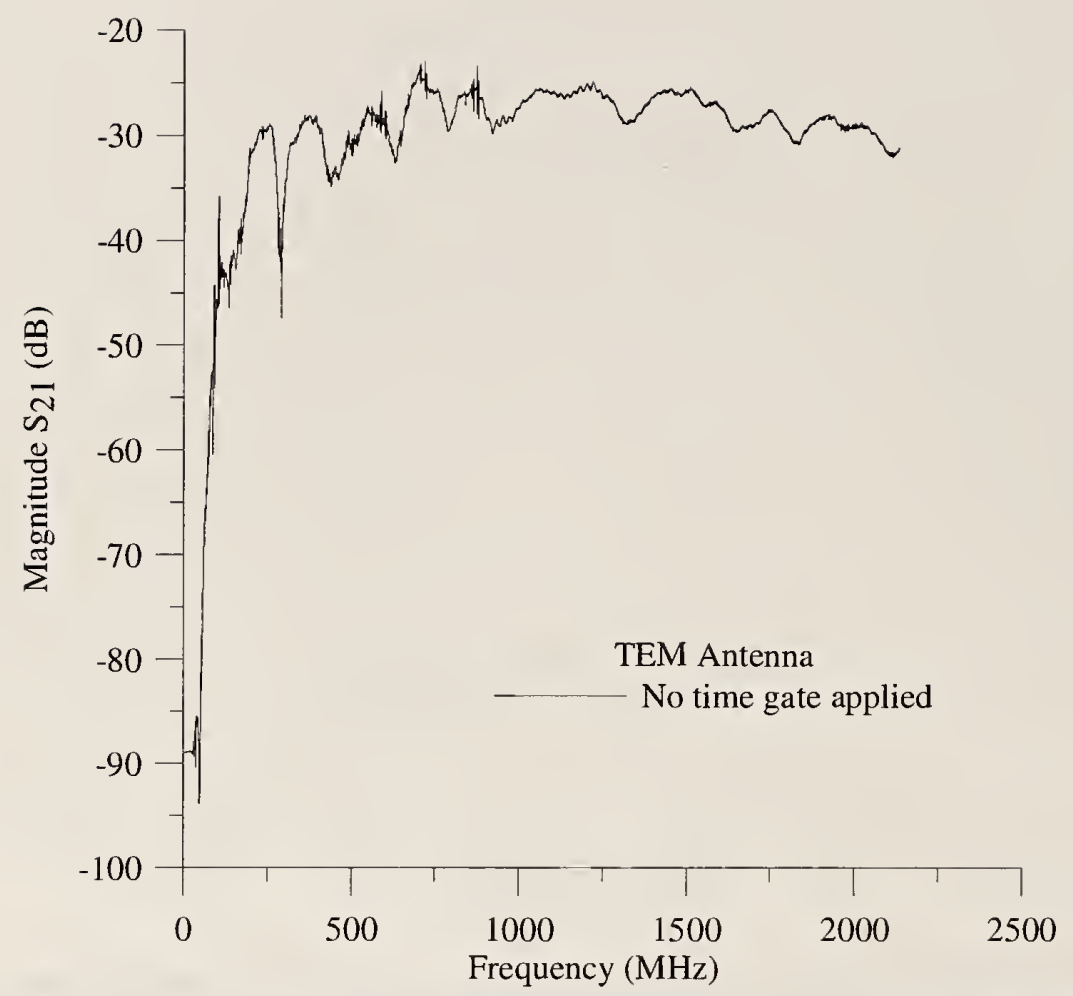

Figure 12. Ungated frequency-domain amplitude spectrum for a TEM horn antenna obtained at a separation of $6 \mathrm{~m}$ and vertical polarization. Note the scalloping due to the large ground bounce reflection shown in Figure 11.

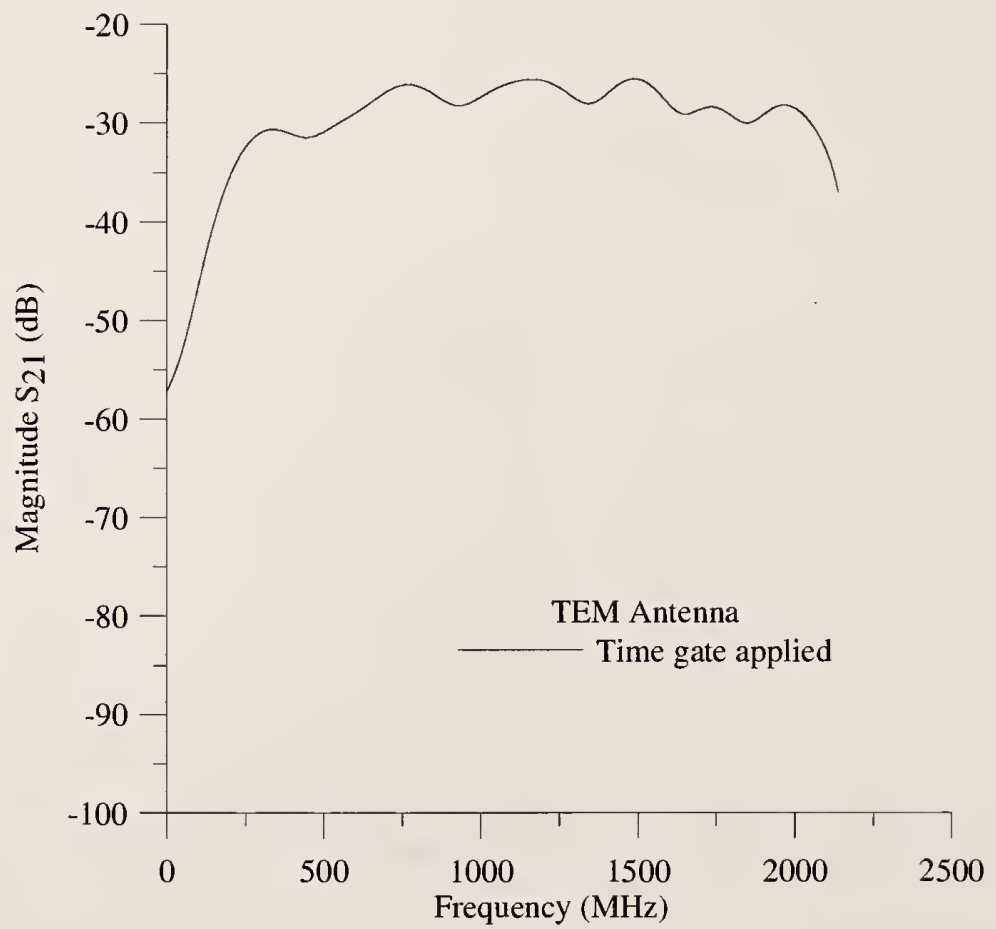

Figure 13. Gated frequency-domain amplitude spectrum corresponding to the waveform of Figure 12. 


\section{Airframe Penetration Measurements-Overview}

We began by measuring the penetration at several extrapolation points on the $45^{\circ}$ line as shown by the black X's in Figure 14. For penetration measurements, the external transmitting antennas were boresighted at the geometric center of the aircraft and moved to specific clock positions at a radial distance of $23 \mathrm{~m} \mathrm{(75} \mathrm{ft)} \mathrm{as} \mathrm{shown} \mathrm{by} \mathrm{the} \mathrm{blue,} \mathrm{dashed} \mathrm{line} \mathrm{in} \mathrm{Figure} \mathrm{14.} \mathrm{We} \mathrm{applied}$ antenna gain and distance correction factors to maintain consistency in the results. The receiving antenna was placed in three compartments of the aircraft: (1) the flight deck, (2) the main cabin, and (3) the rear cargo hatch. These locations are shown by the red +'s.

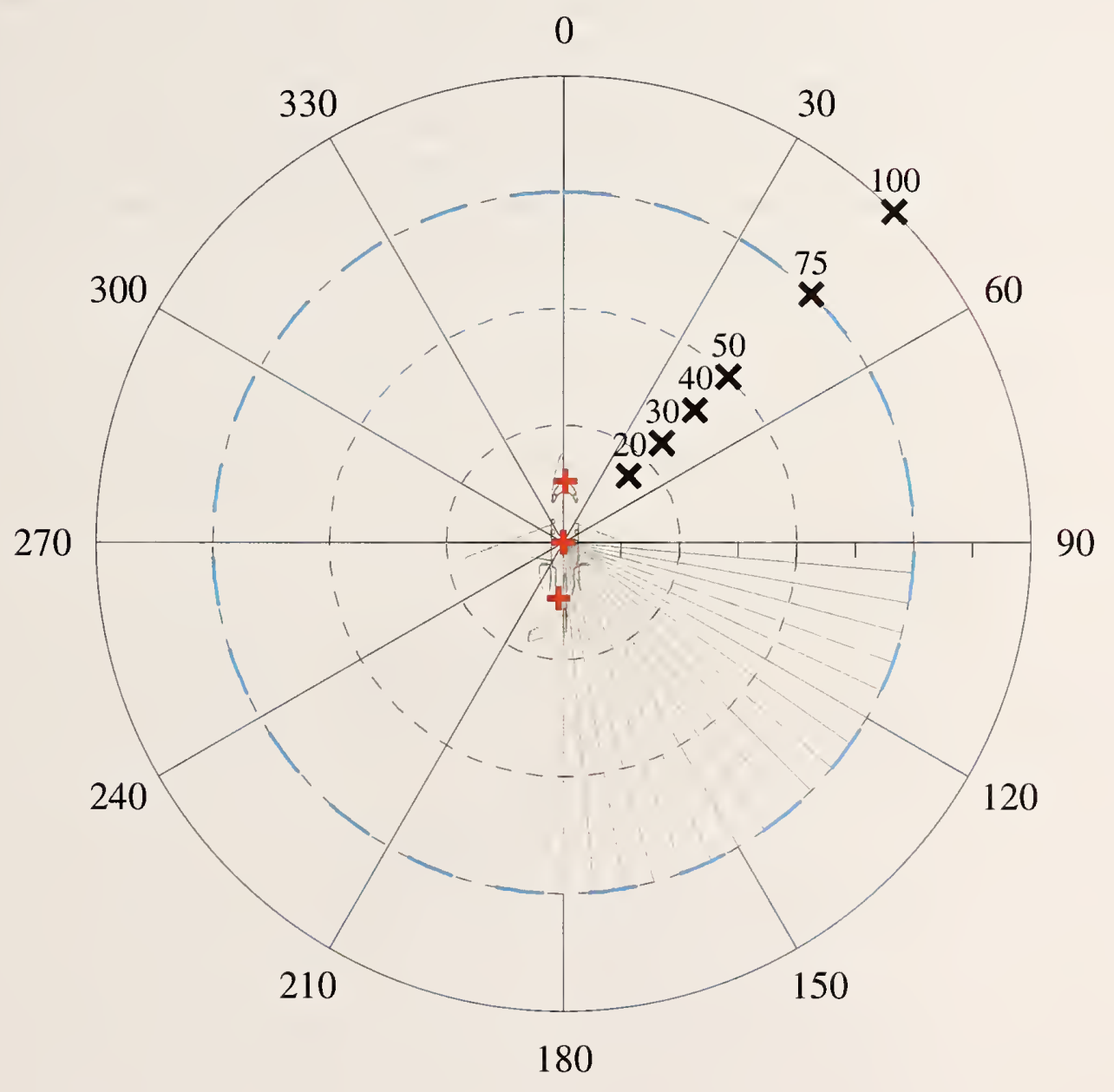

Figure 14. Test master graphic showing internal measurement positions (red +'s), Extrapolation distances in feet (black X's), measurements around aircraft (blue, dashed line) at a distance of $23 \mathrm{~m}(75 \mathrm{ft})$. 


\subsection{Extrapolation Results}

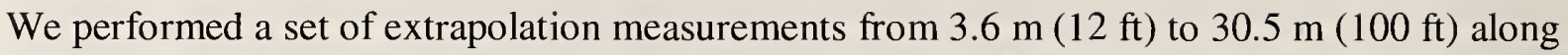
the $45^{\circ}$ line to investigate the far-field behavior of the aircraft and to understand how penetration changes as a function of distance from the aircraft. Distance correction was applied to the data by use of a reference distance of $6.1 \mathrm{~m}$. These measurements are shown in Figures 15 to 18 . The data show a spread of around $10 \mathrm{~dB}$ across the frequency spectrum for both the DRG and TEM horn antennas. We believe this is because, at the closer distances, our antennas see more of the aircraft, but as we move further from the aircraft the antennas see less of the aircraft and more of the surrounding environment. In Figure 16, if we remove the $3.6 \mathrm{~m}$ data, the $6.1 \mathrm{~m}$ data, and the $9.1 \mathrm{~m}$ data, then the spread in the data is between $2 \mathrm{~dB}$ and $3 \mathrm{~dB}$ for the DRG horn antennas. If we remove the $3.6 \mathrm{~m}$ and $6.1 \mathrm{~m}$ data from Figures 17 and 18 , then the TEM horn extrapolation results show spreads in the data on the order of $2 \mathrm{~dB}$ to $5 \mathrm{~dB}$. We note here that the DRG antennas have higher directivity than that of the TEM horn antennas, which corresponds to the greater spread in penetration data for the TEM horn antenna. These results have been corrected for both distance and antenna gain. The gains for the DRG horn antenna and the TEM horn antenna are discussed in Appendix C.

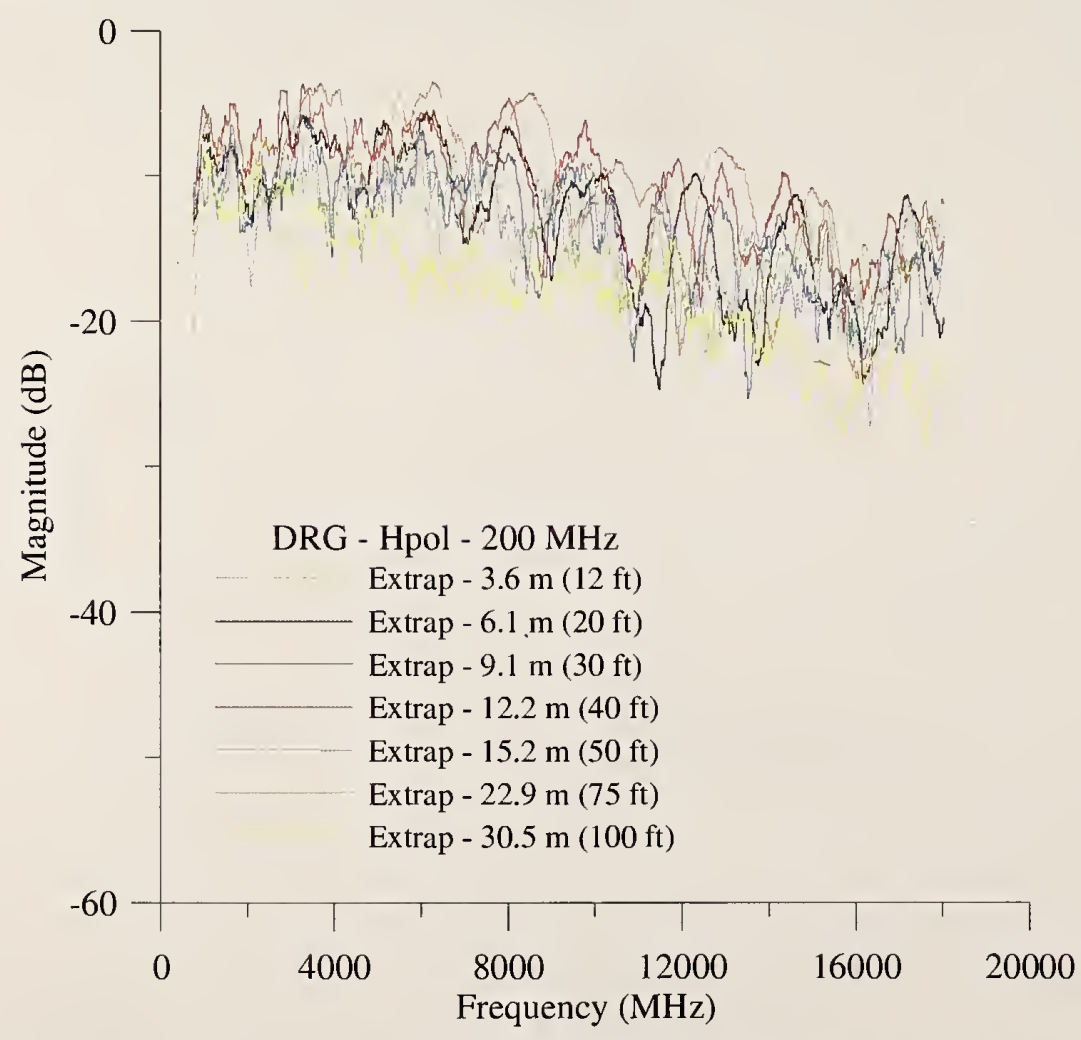

Figure 15. Extrapolation measurements for the DRG antenna, horizontal polarization. 


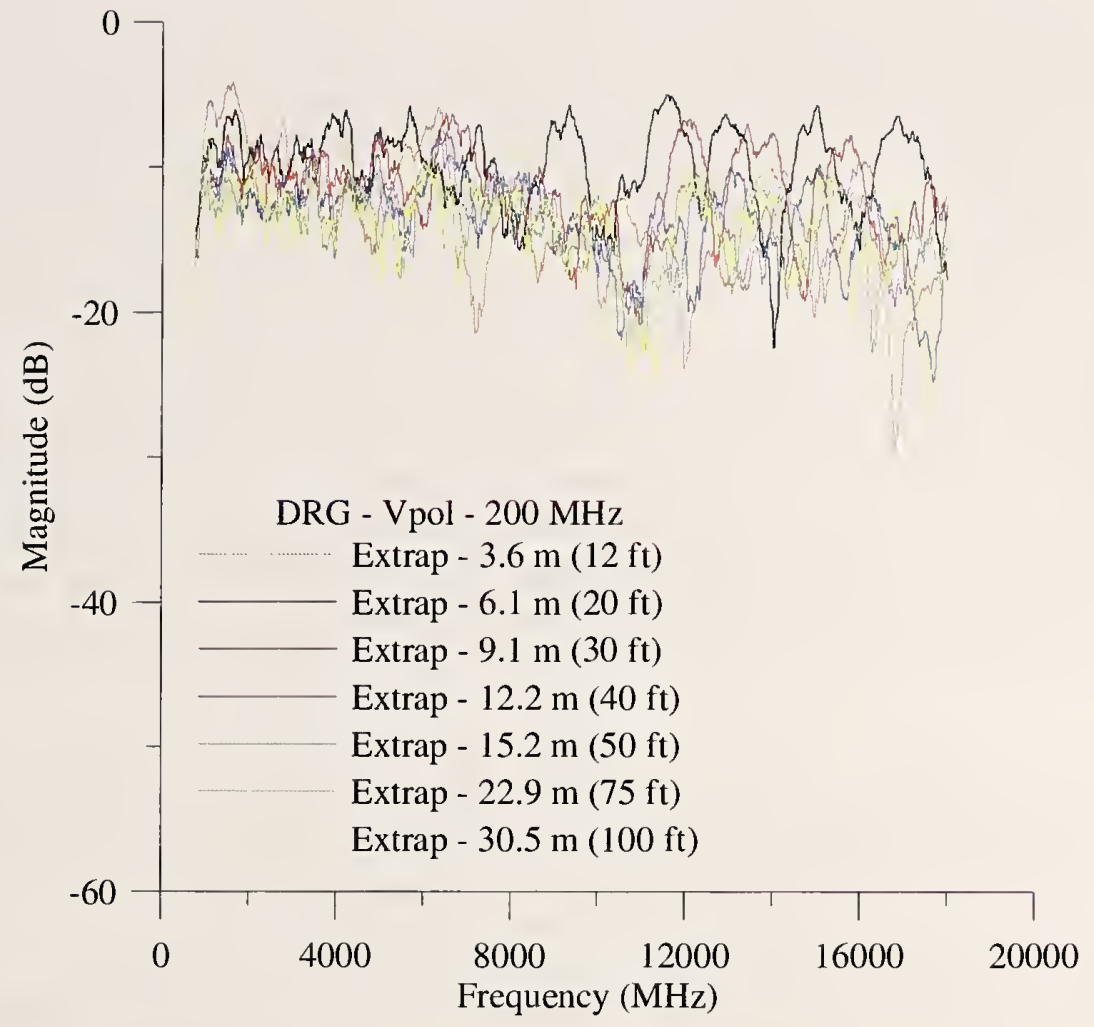

Figure 16. Extrapolation measurements for the DRG antenna, vertical polarization.

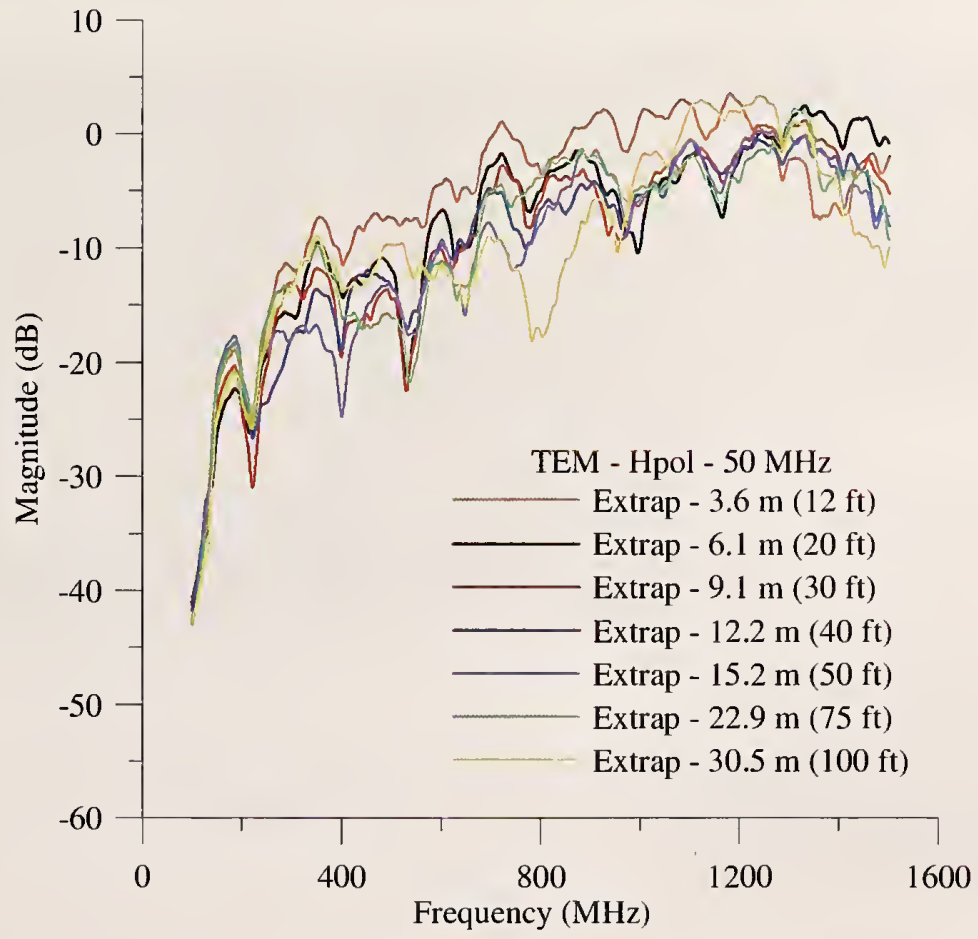

Figure 17. Extrapolation measurements for the TEM antenna, horizontal polarization. 


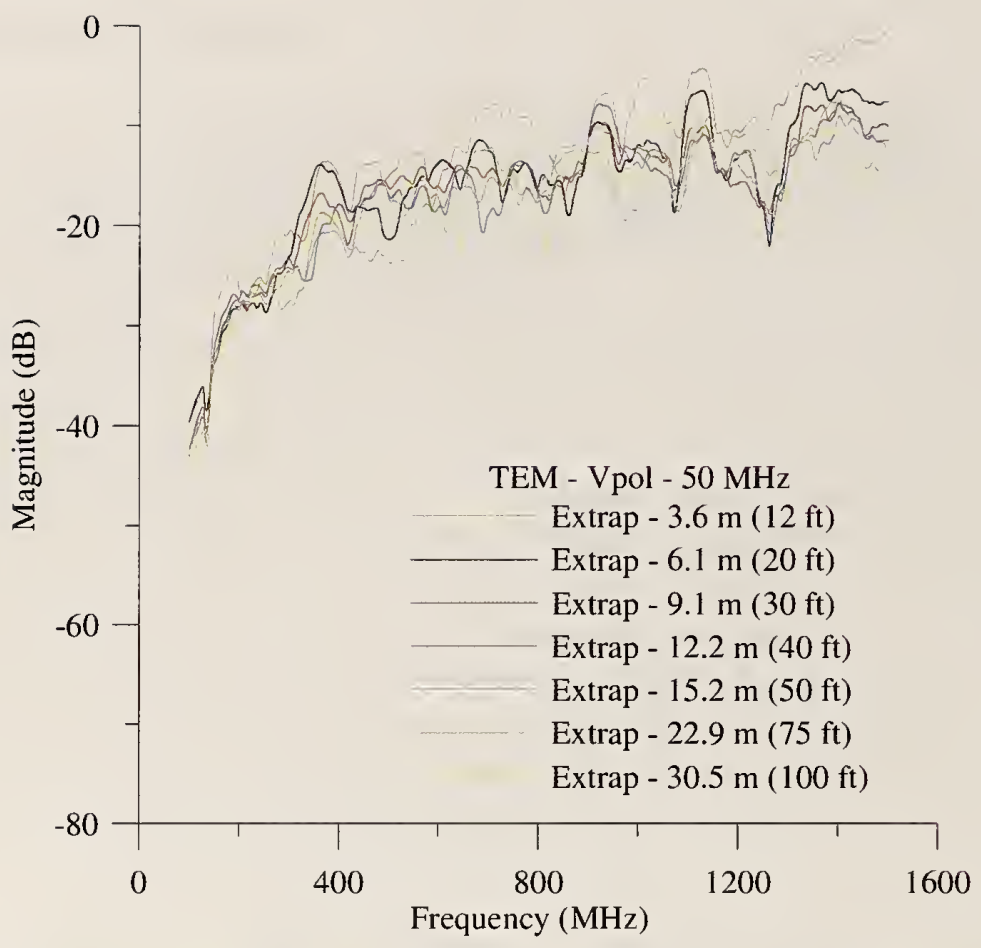

Figure 18. Extrapolation measurements for the TEM antenna, vertical polarization.

\subsection{Main Passenger Cabin Penetration Results}

The receiving antenna was located near the geometric center of the main cabin, as shown in Figure 19. The antennas were placed near the center of the aircraft and were mounted on tripods to provide support and separation. The antenna position was selected to provide robust excitation of available cavity modes. The optical fiber was fed through one of the passenger windows on the left side of the aircraft, which remained partially propped open for the main cabin and flight deck measurements. We are assuming the effects from this small aperture are minimal. The transmitting antennas were boresighted to the geometric center of the aircraft to avoid assumptions about signal penetration apertures.

Time-domain plots are used as an aid to understand penetration into airframes. We begin by transforming the measured frequency domain data into the time-domain to look at the signal in time. Time-domain plots for the DRG antenna in the horizontal polarization are shown in Figure 20 at various angular positions around the aircraft. At an angular position of $0^{\circ}$, we have a moderate, initial sharp impulse response (the direct antenna-to-antenna coupling component), which decays rapidly within $150 \mathrm{~ns}$. This is indicative of a rapidly decaying reverberant field. Another, much smaller, signal appears at around $400 \mathrm{~ns}$, which we believe is a signal coming from the surrounding environment. As we travel around the aircraft we obtain a maximum 
signal at an angular position of $45^{\circ}$ decreasing in level until we get to the tail position at $180^{\circ}$. In typical aircraft, we see very low signal amplitudes toward the tail of the aircraft. For this aircraft, we see similar levels as we traverse around the aircraft, so we believe that the signal is able to penetrate somewhat equally into the main passenger cabin from all angular positions. The larger the direct coupling component, the more energy there is that initially gets coupled into the main passenger cabin and the less energy available in the reverberant field. A more in-depth discussion of the characteristics of these waveforms is provided in Appendix A. This timedomain analysis provides valuable insight into possible maximum coupling paths. Time-domain plots for the DRG antenna in the vertical polarization are shown in Figure 21 and time-domain plots for the TEM horn antennas in both polarizations are shown in Figures 22 and 23. The TEM horn antennas receive lower signal levels, but there is still time-domain evidence of direct coupling. Although the TEM horn antennas have low directive gain (broad radiation pattern) the available penetration from any part of the aircraft allows the antenna to pick up the direct component.

Frequency-domain plots for various positions around the aircraft are summarized in Figure 24. The purpose of this plot is to show a snapshot of the variation in penetration level at selected positions around the aircraft. In a typical aircraft we would expect the signal to drop at the front and back of the aircraft due to lack of direct signal paths at these positions; however, this aircraft does not show this behavior. We have also plotted some penetration results at various symmetrical positions around the aircraft to determine whether there may be leakage points on one side that are not on the other. This is shown in Figure 25. We would expect a possible leakage point at the door into the aircraft. In Figure 24, we see that the maximum penetration of about $0 \mathrm{~dB}$ occurs around $900 \mathrm{MHz}$ for the DRG antennas, with the transmitting antenna in the vertical and horizontal polarizations, at an angle of $30^{\circ}$, and the receiving antennas in the main

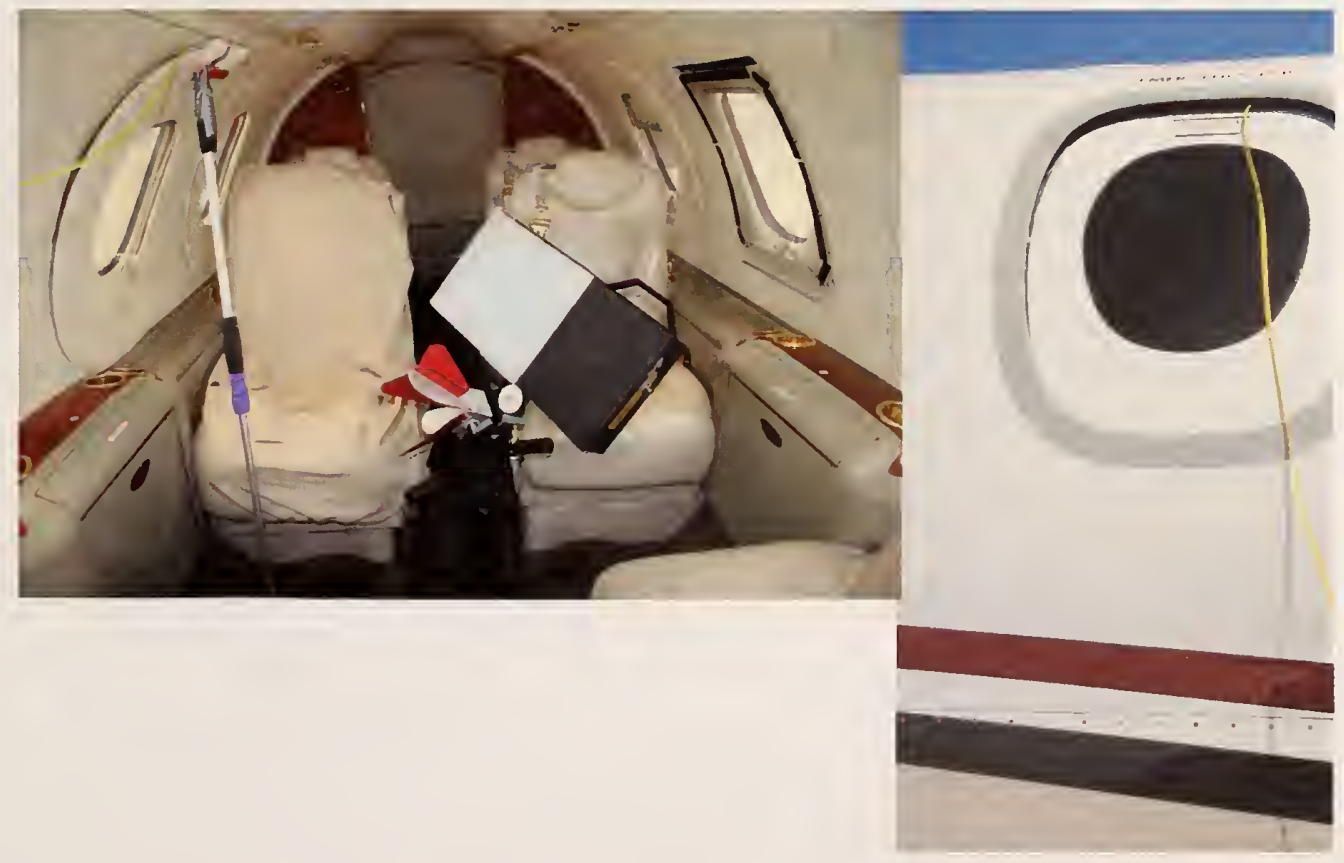

Figure 19. Receiving antenna placement in main passenger cabin. 
passenger cabin. Minimum penetration of approximately $-30 \mathrm{~dB}$ occurs at an angle of $180^{\circ}$ for the DRG antenna in the horizontal and vertical polarizations. For TEM horn antennas with signals below $100 \mathrm{MHz}$, we find that the penetration artificially increases, which is an artifact of the measurement system rapidly losing dynamic range. This reduction in dynamic range is due to combination of increasing airframe shielding performance and reduced antenna efficiency.

Detailed penetration results at each azimuthal position for all antennas and all polarizations are summarized in Figures 26 to 50. The DRG antenna data are frequency averaged over a bandwidth of $200 \mathrm{MHz}$, and the TEM horn antenna data are frequency averaged over a bandwidth of $50 \mathrm{MHz}$. We have concluded that these bandwidths work well at suppressing the "hashy" behavior due to the complex cavity modes while emphasizing the deterministic coupling effects. Frequency averaging is described in detail in references [1-3]. We see that the highest penetration occurs at the $30^{\circ}$ position for frequencies around $900 \mathrm{MHz}$. The penetration increases as we proceed to the tail of the aircraft, with maximum values of around $-30 \mathrm{~dB}$. This directly correlates to the time domain data discussed previously. The horizontally polarized antennas tend to have higher penetration values than the vertically polarized antennas, which could be due to the horizontally polarized array of windows around the aircraft. Our purpose in moving every $10^{\circ}$ until we reach the second quadrant and then changing to $5^{\circ}$ increments is to investigate the possibility that we may be missing important leakage points by only traversing every $10^{\circ}[4]$. What we see is that the angular variation in penetration does not change greatly from position to position, with typical variations less than $5 \mathrm{~dB}$. This can be attributed to a combination of complex cavity behavior and the possible coupling paths into the main passenger cabin. Due to the large number of coupling apertures, we do not take into account the antenna pattern effects for either the antennas or the aircraft. If we knew exactly where the fields were penetrating into the cavity, then these types of corrections would be feasible. These coupling paths are both vertically and horizontally polarized, and therefore we believe the fields inside the aircraft are depolarized to a large extent.

The main conclusions for an antenna placed in the main passenger cabin are: (1) main coupling apertures are the windows on the flight deck, (2) penetration is slightly lower at the tail of the aircraft, (3) penetration into the airframe is fairly symmetric and of the same magnitude around the aircraft, (4) typical penetration values are $0 \mathrm{~dB}$ at $30^{\circ}$ and $-30 \mathrm{~dB}$ for the $180^{\circ}$ positions, and (5) the horizontally polarized energy more easily penetrates the airframe than the vertically polarized energy. We believe this is because of the horizontal "slot apertures" that the array of windows provides. 


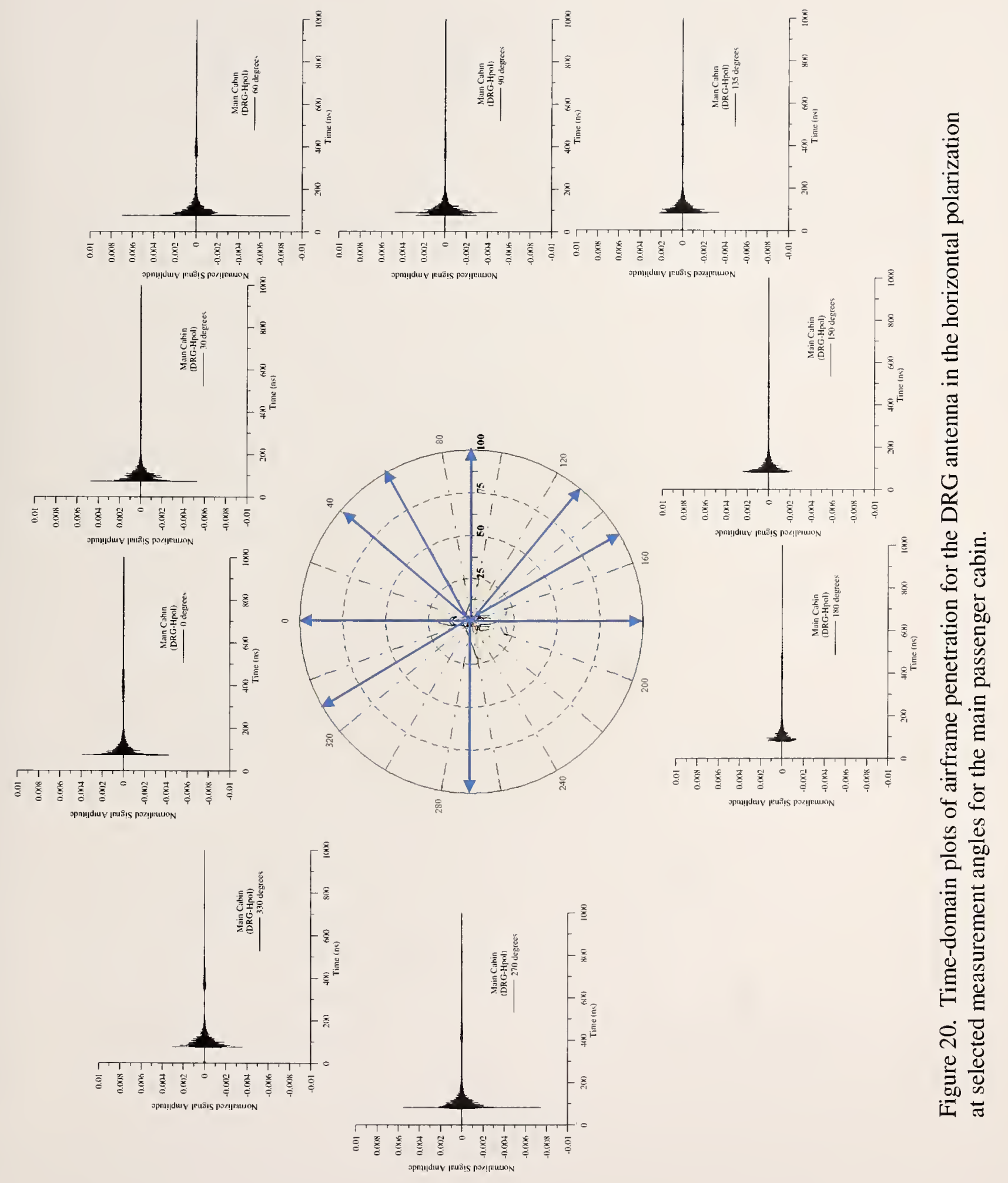




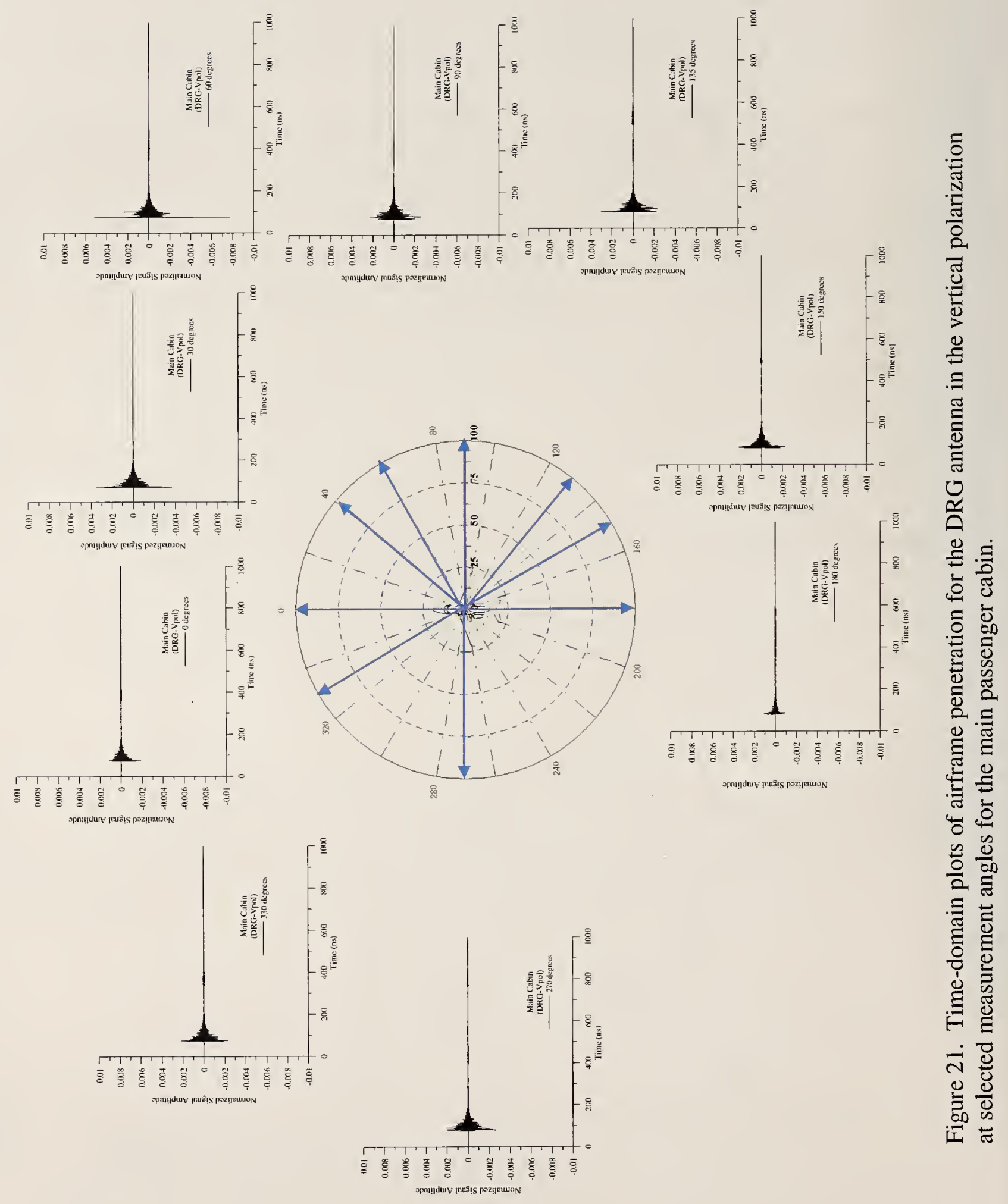




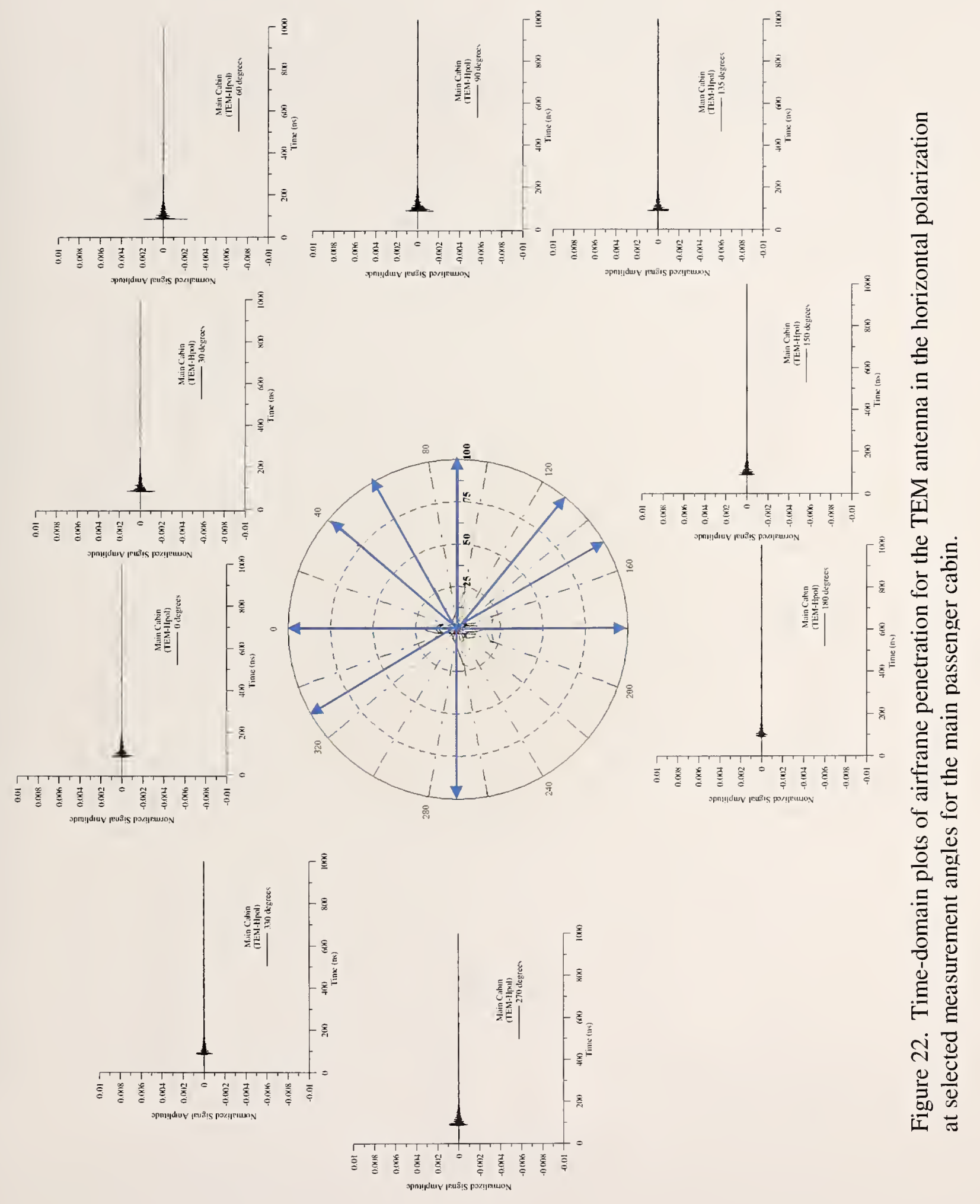




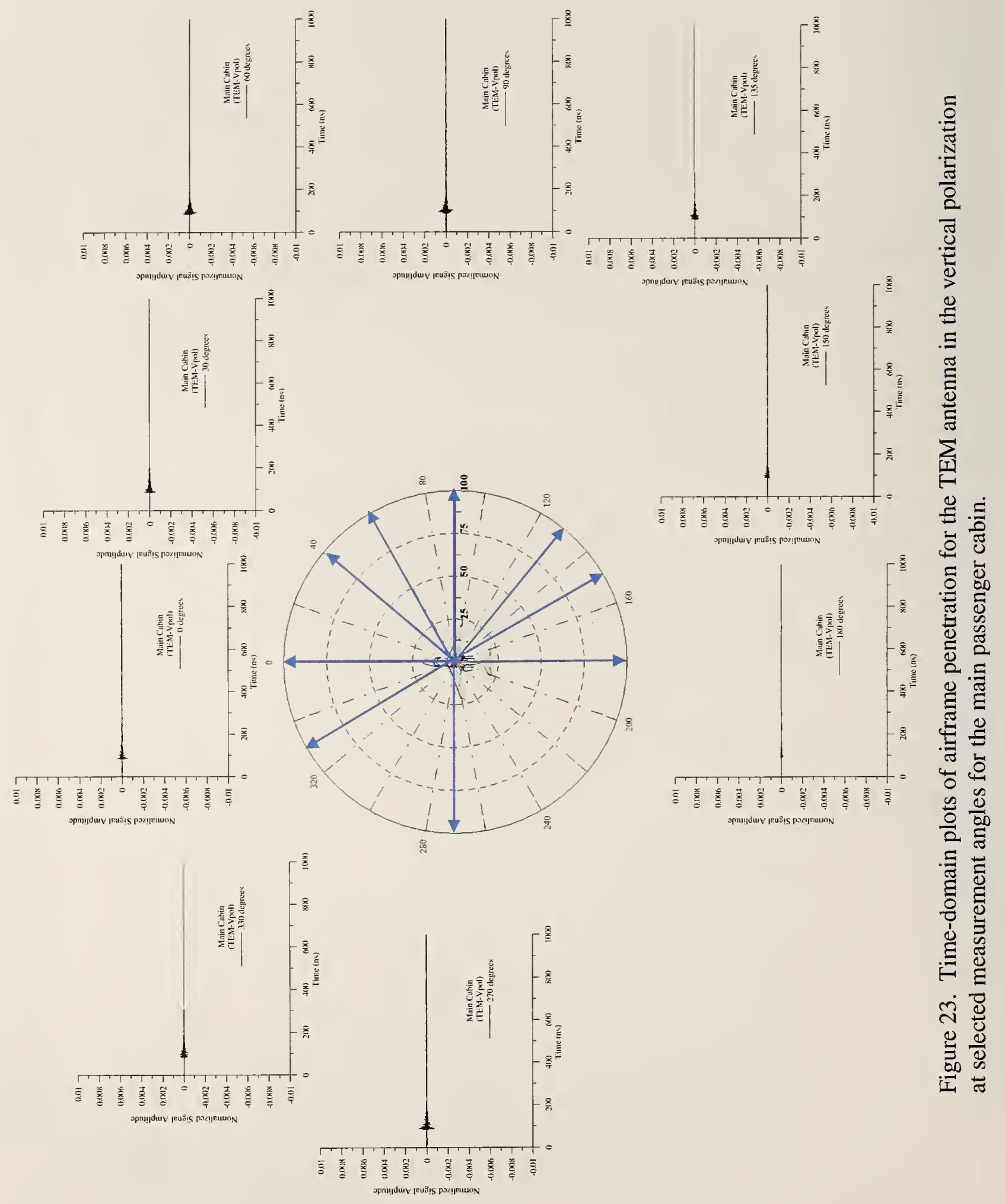




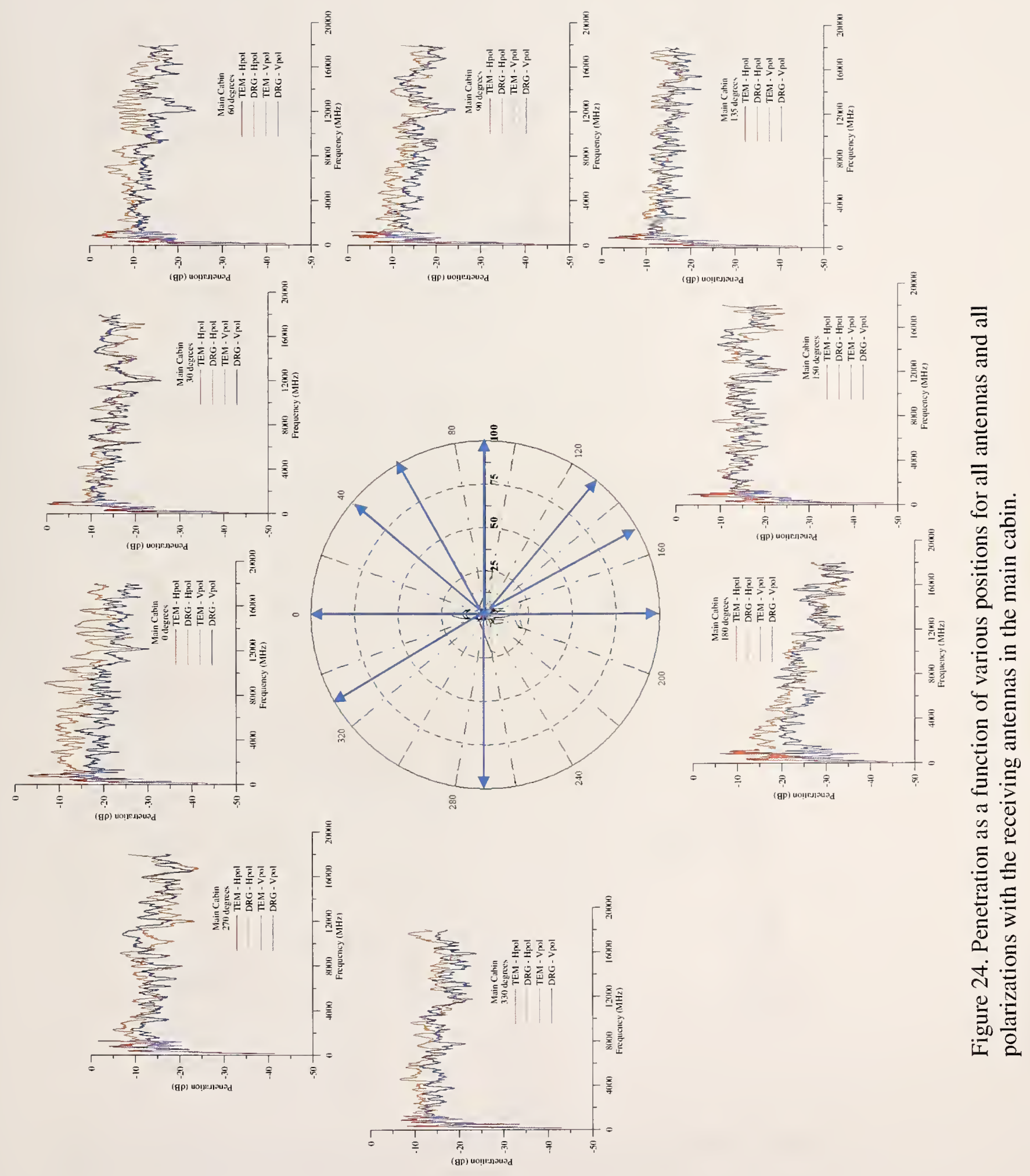




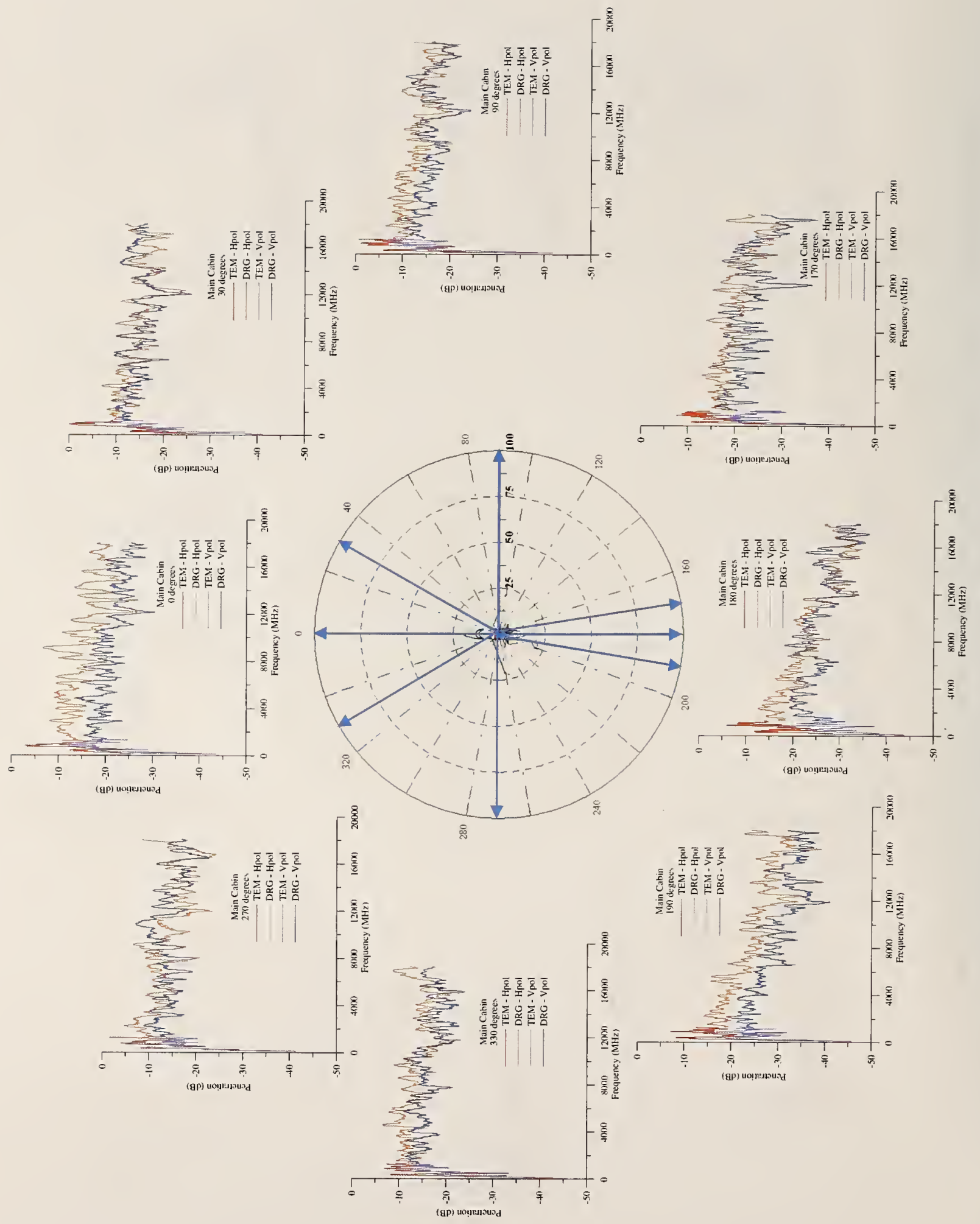



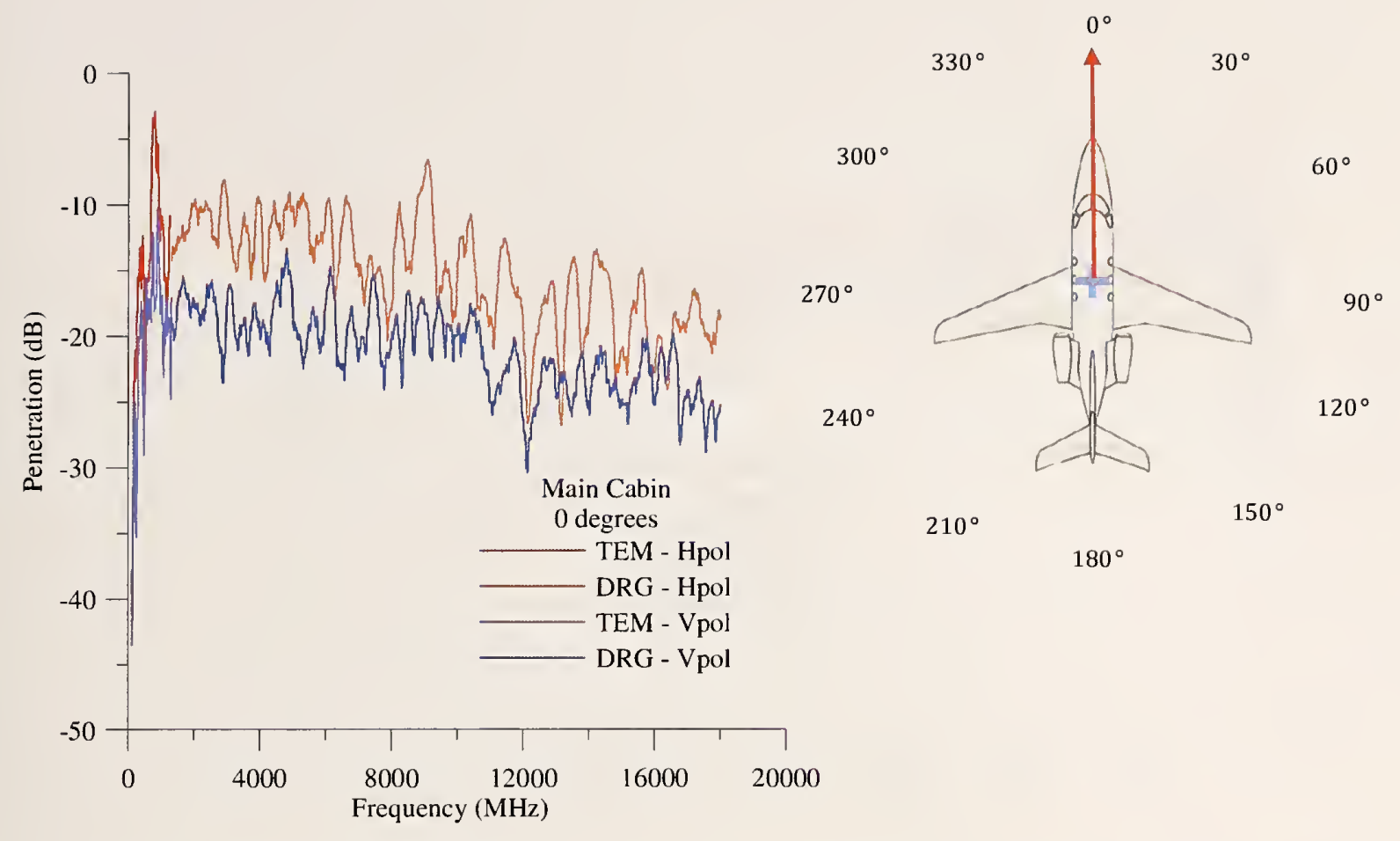

Figure 26. Penetration for all antennas and polarizations for the transmitting tower placed at the $0^{\circ}$ position and the receiving antennas placed in the main cabin.

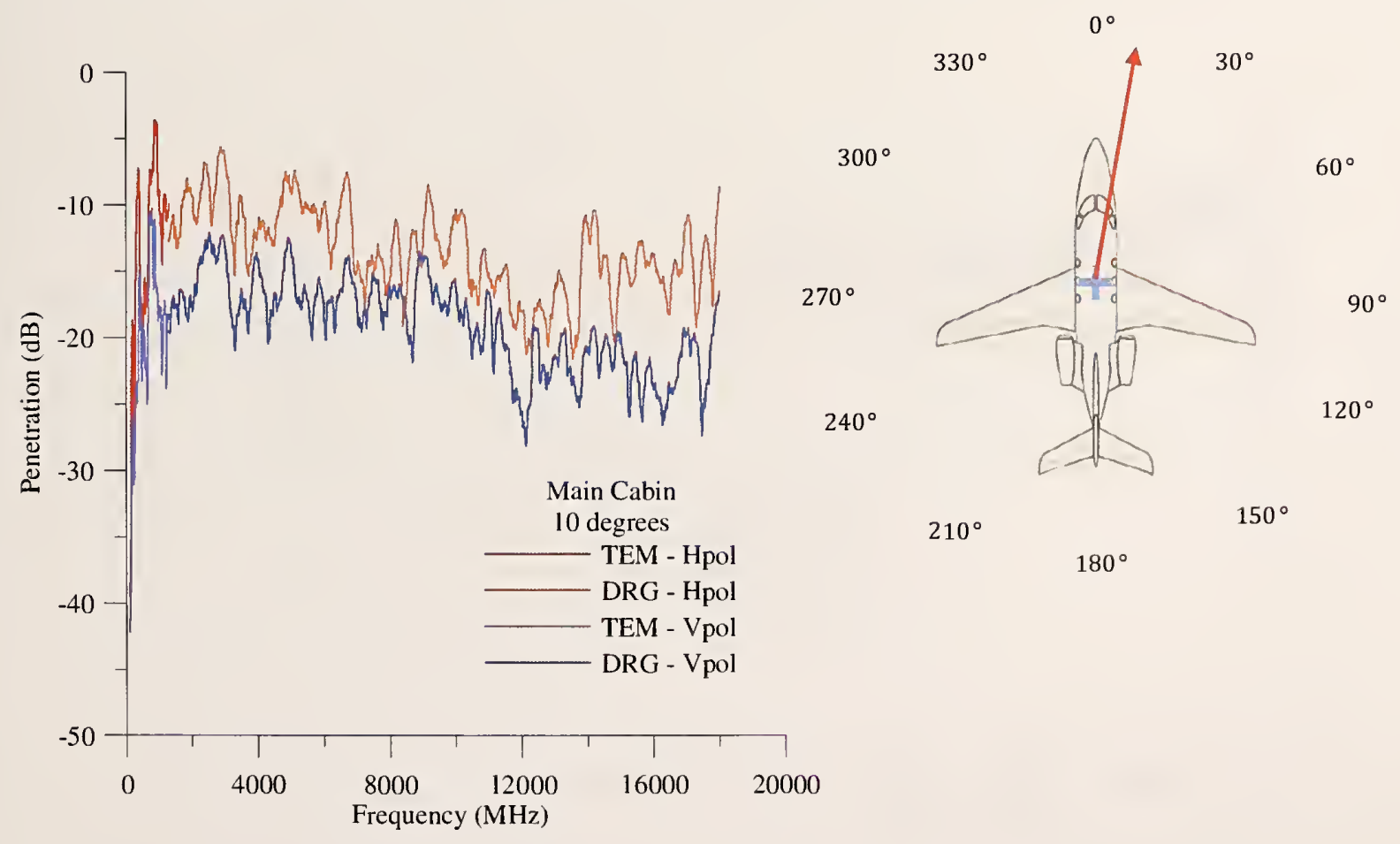

Figure 27. Penetration for all antennas and polarizations for the transmitting tower placed at the $10^{\circ}$ position and the receiving antennas placed in the main cabin. 


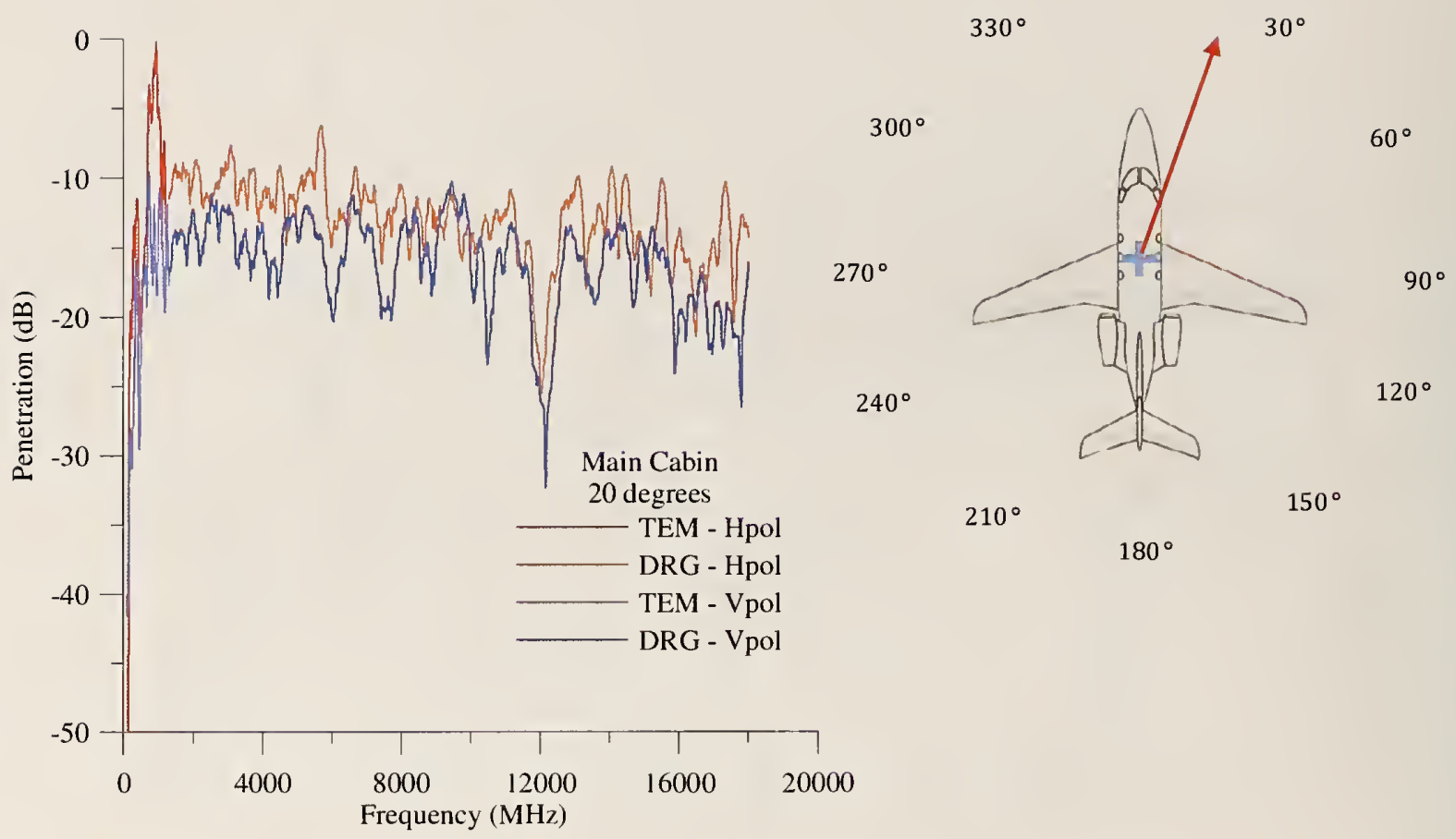

Figure 28. Penetration for all antennas and polarizations for the transmitting tower placed at the $20^{\circ}$ position and the receiving antennas placed in the main cabin.

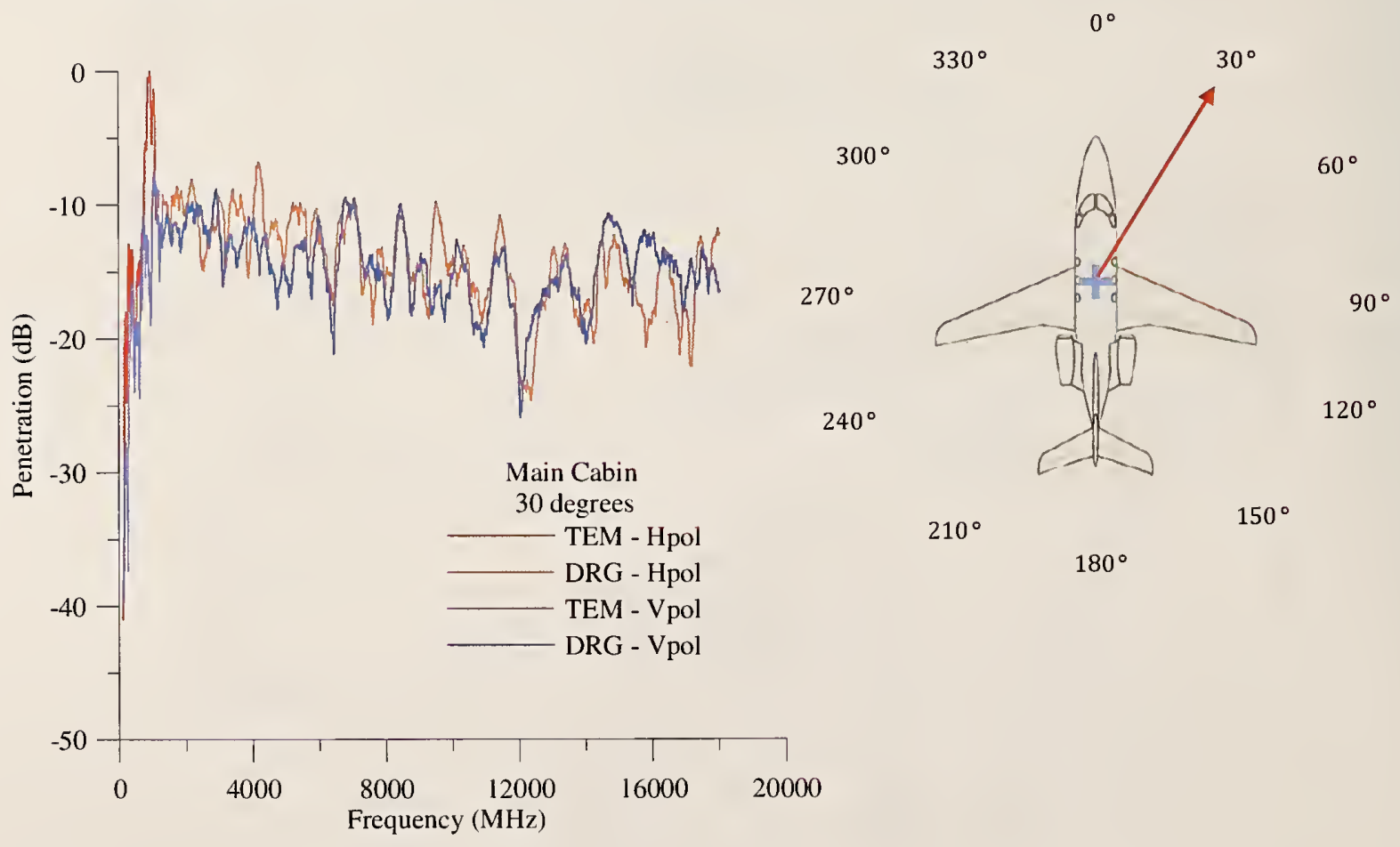

Figure 29. Penetration for all antennas and polarizations for the transmitting tower placed at the $30^{\circ}$ position and the receiving antennas placed in the main cabin. 


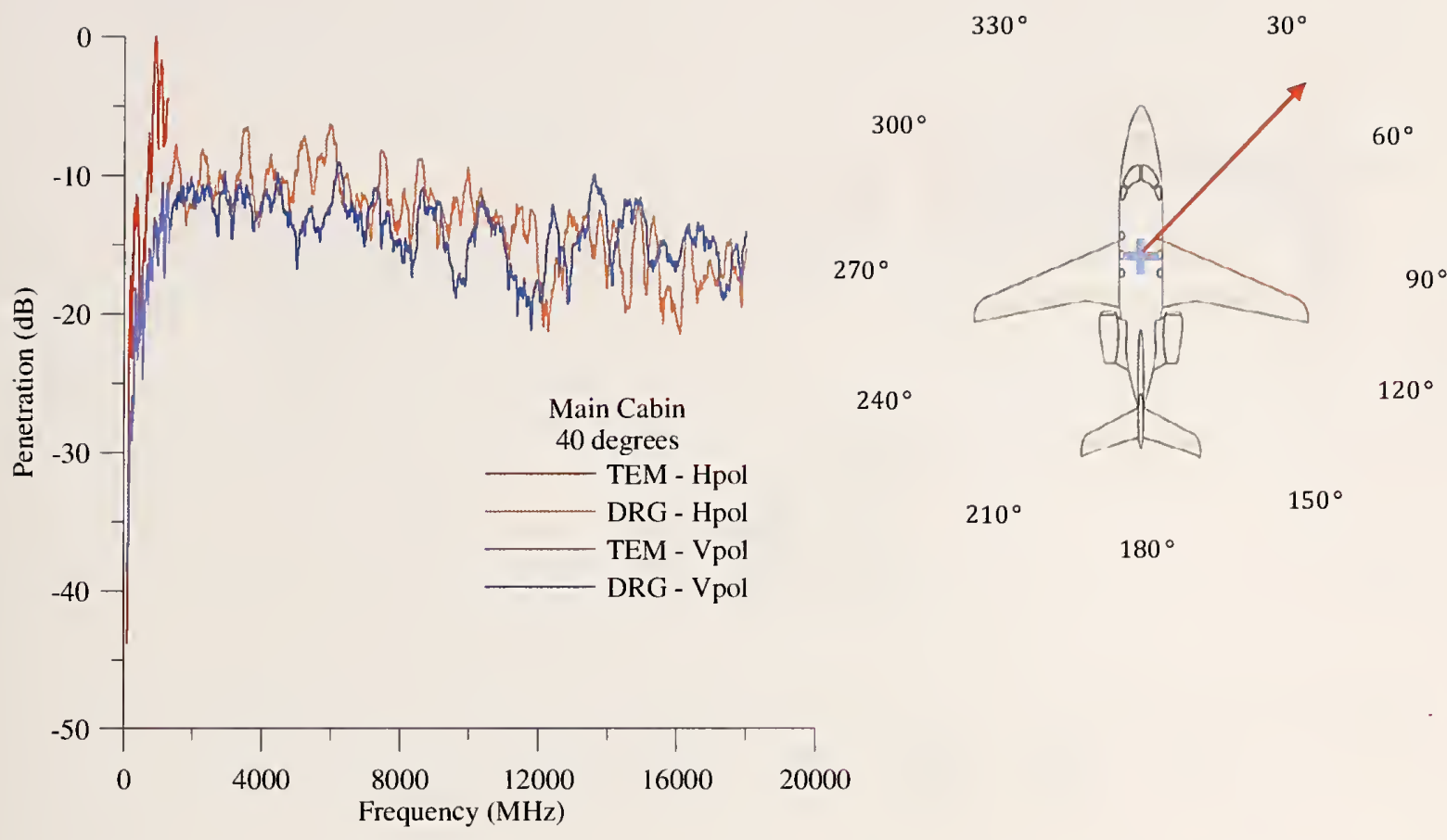

Figure 30. Penetration for all antennas and polarizations for the transmitting tower placed at the $40^{\circ}$ position and the receiving antennas placed in the main cabin.

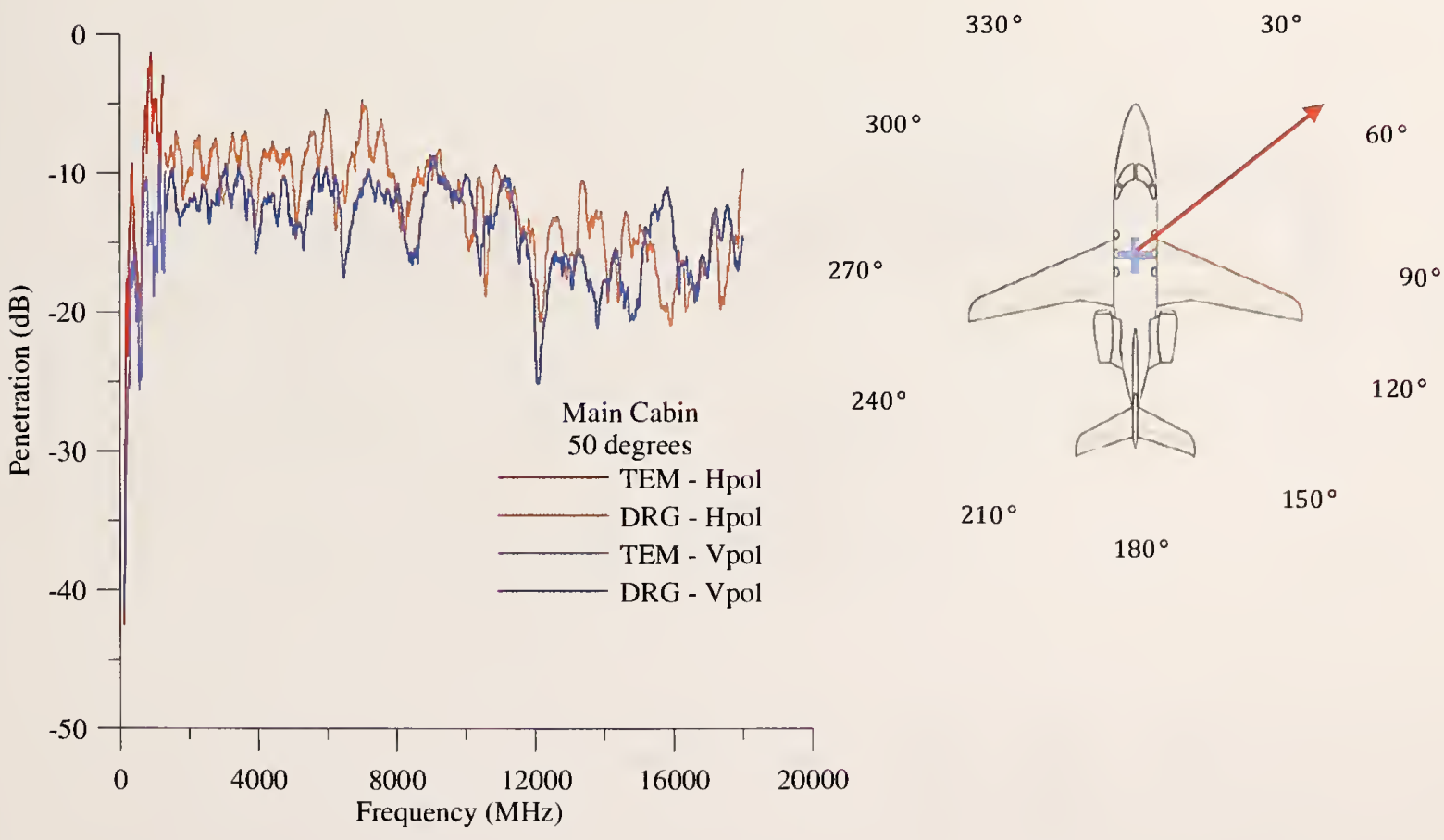

Figure 31 . Penetration for all antennas and polarizations for the transmitting tower placed at the $50^{\circ}$ position and the receiving antennas placed in the main cabin. 


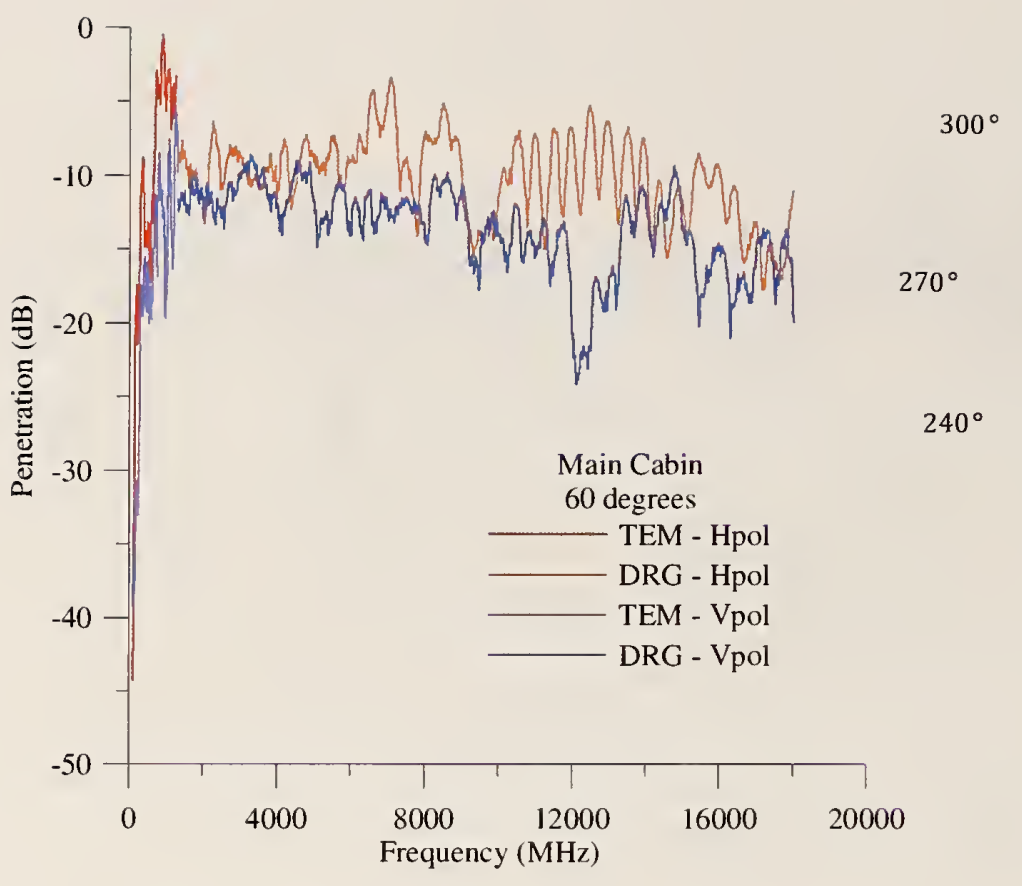

$330^{\circ} \quad 30^{\circ}$

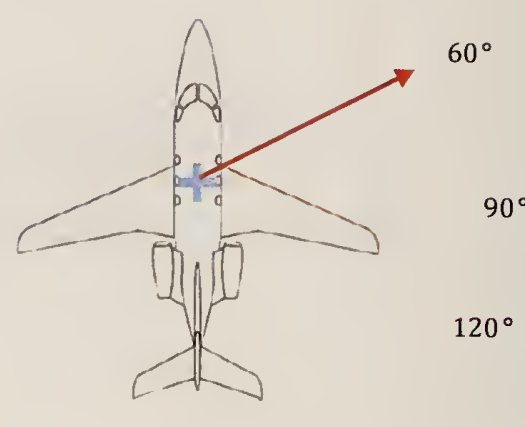

$210^{\circ}$

$150^{\circ}$

$180^{\circ}$

Figure 32. Penetration for all antennas and polarizations for the transmitting tower placed at the $60^{\circ}$ position and the receiving antennas placed in the main cabin.

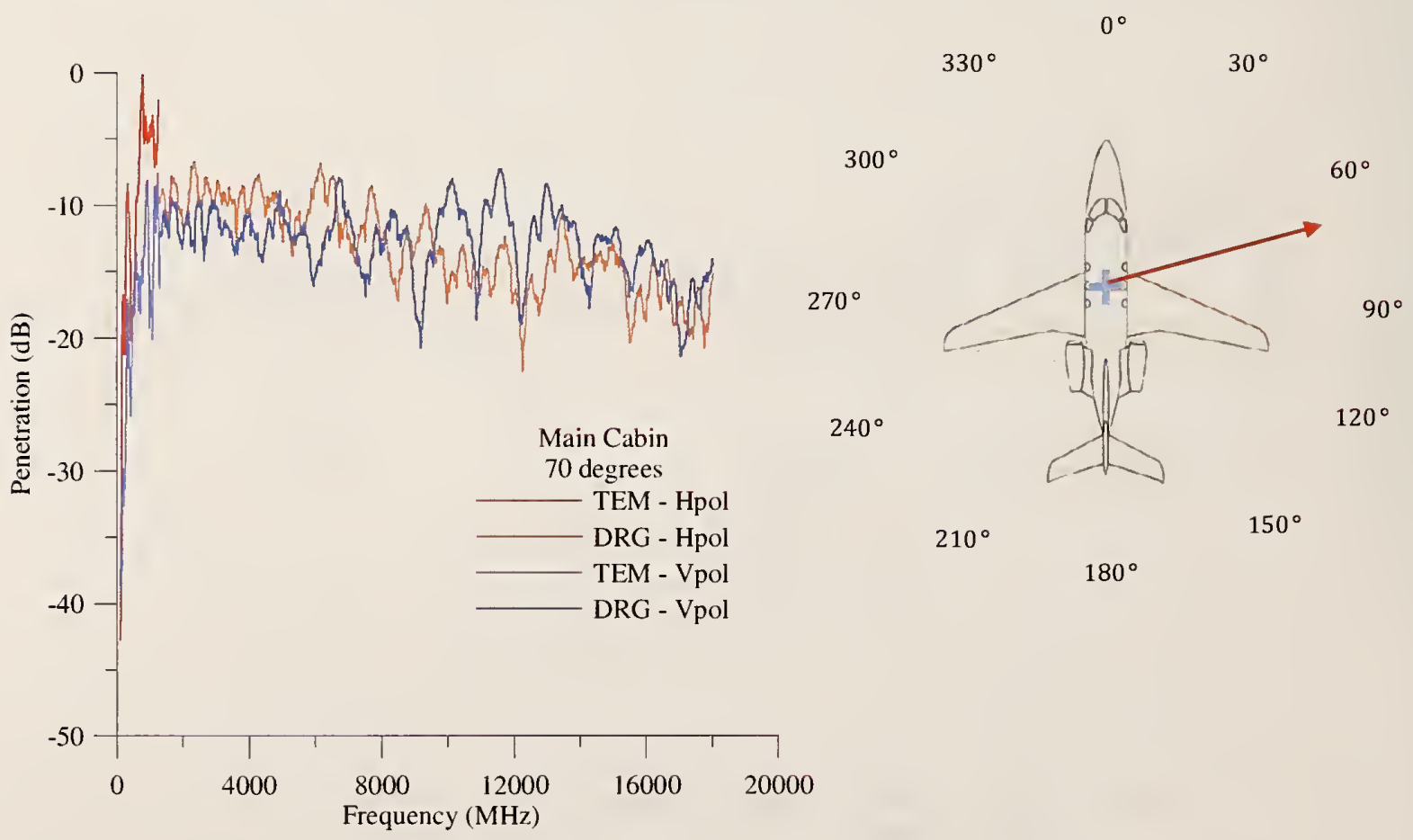

Figure 33. Penetration for all antennas and polarizations for the transmitting tower placed at the $70^{\circ}$ position and the receiving antennas placed in the main cabin. 


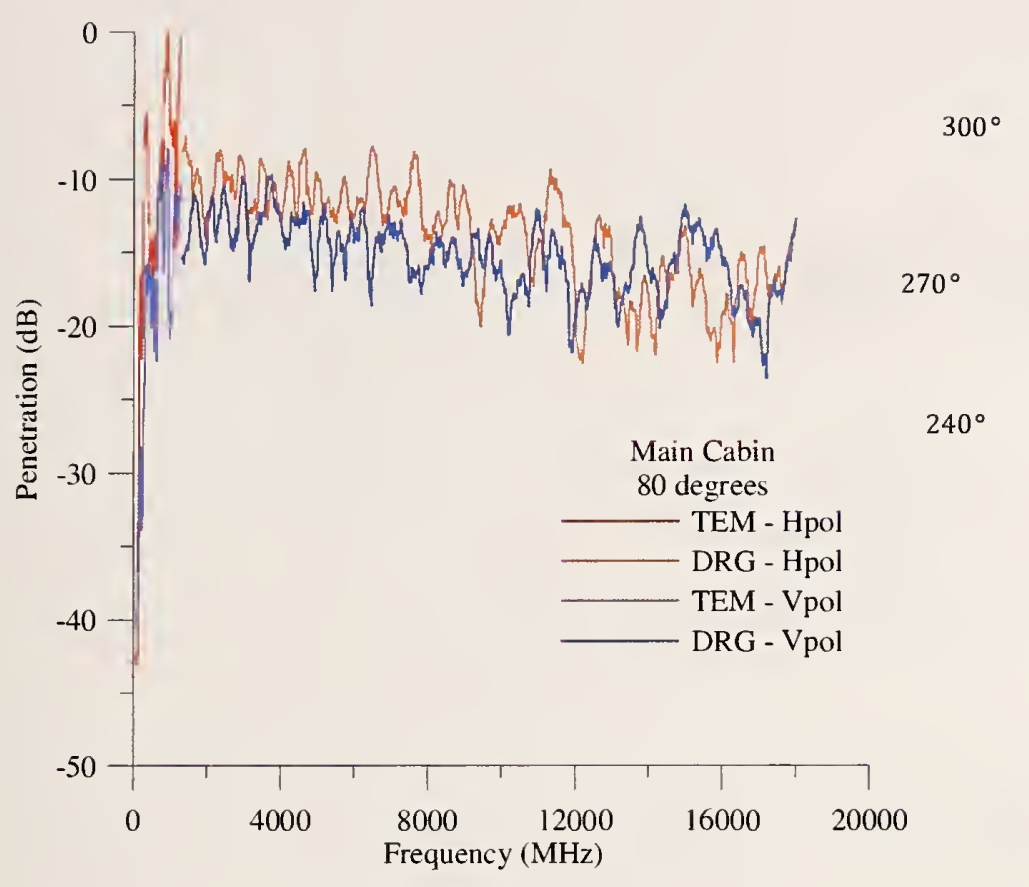

$330^{\circ} 30^{\circ}$

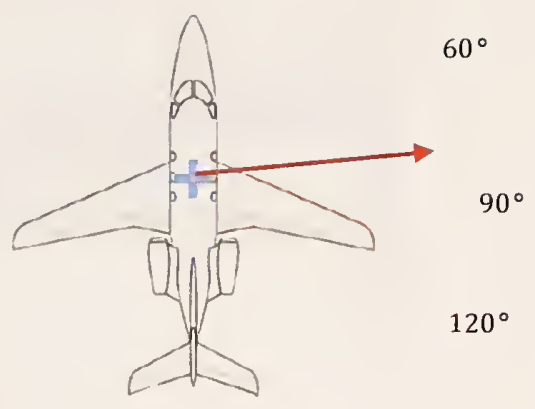

$210^{\circ}$

$150^{\circ}$

$180^{\circ}$

Figure 34. Penetration for all antennas and polarizations for the transmitting tower placed at the $80^{\circ}$ position and the receiving antennas placed in the main cabin.

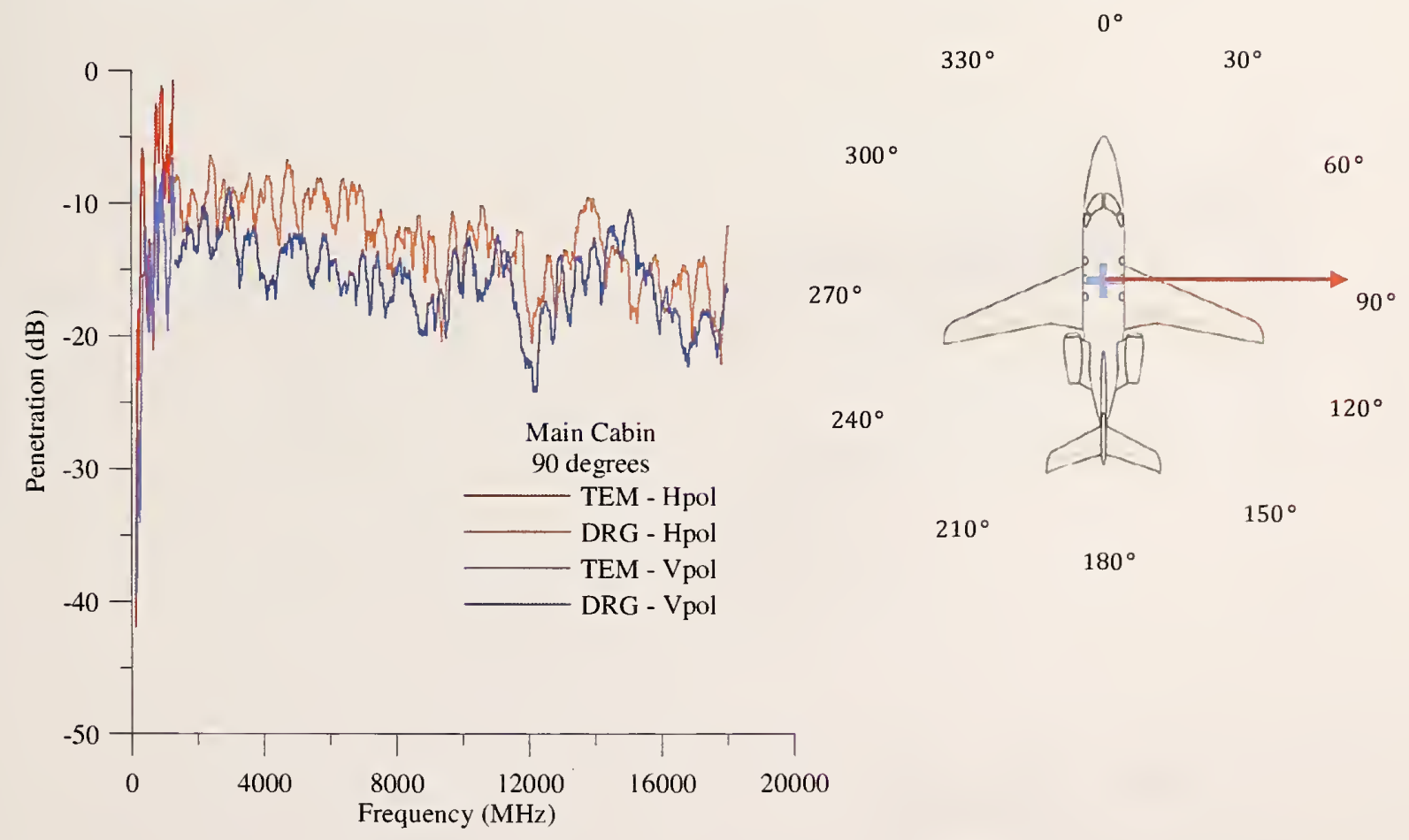

Figure 35. Penetration for all antennas and polarizations for the transmitting tower placed at the $90^{\circ}$ position and the receiving antennas placed in the main cabin. 


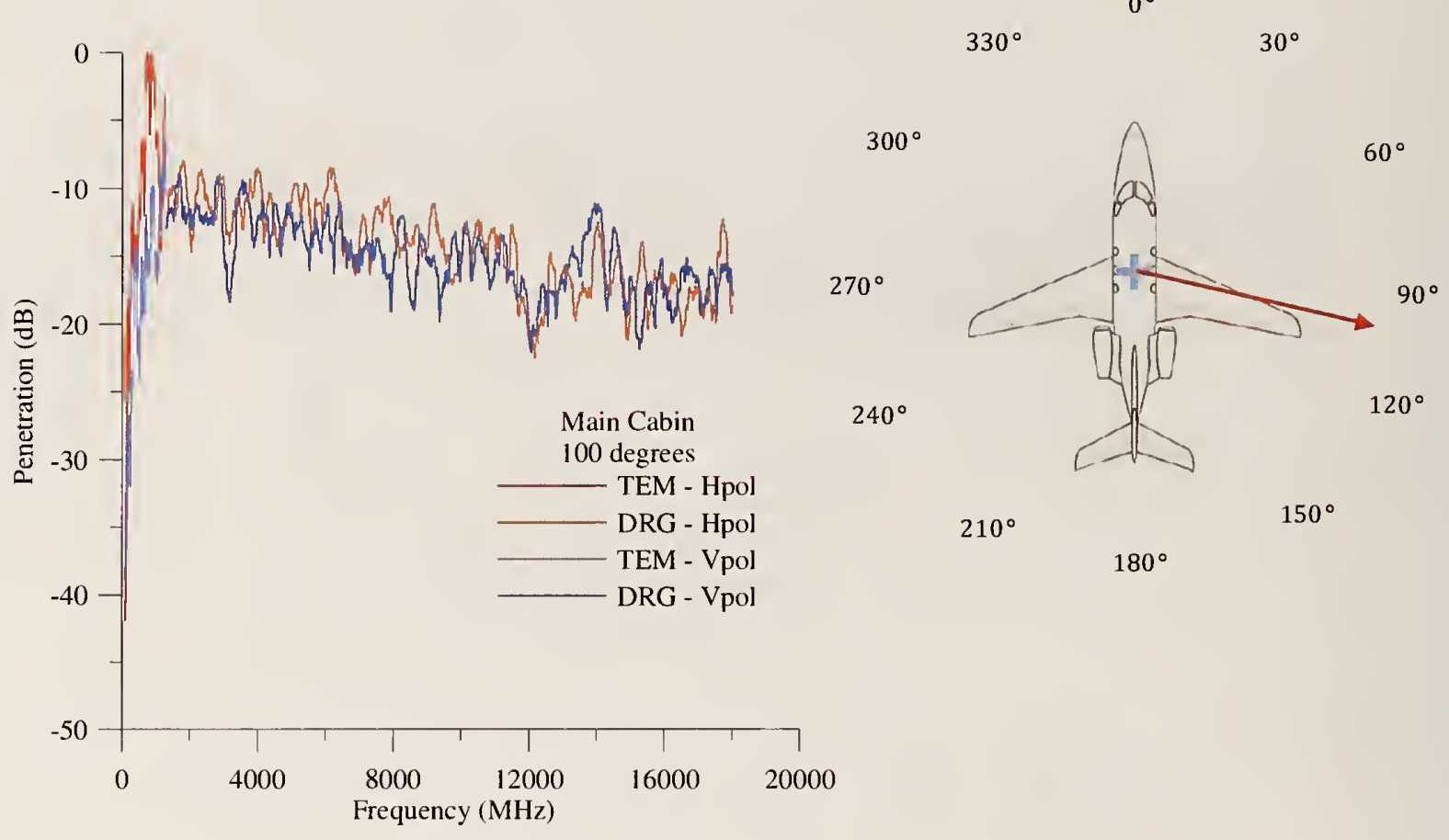

Figure 36. Penetration for all antennas and polarizations for the transmitting tower placed at the $100^{\circ}$ position and the receiving antennas placed in the main cabin.

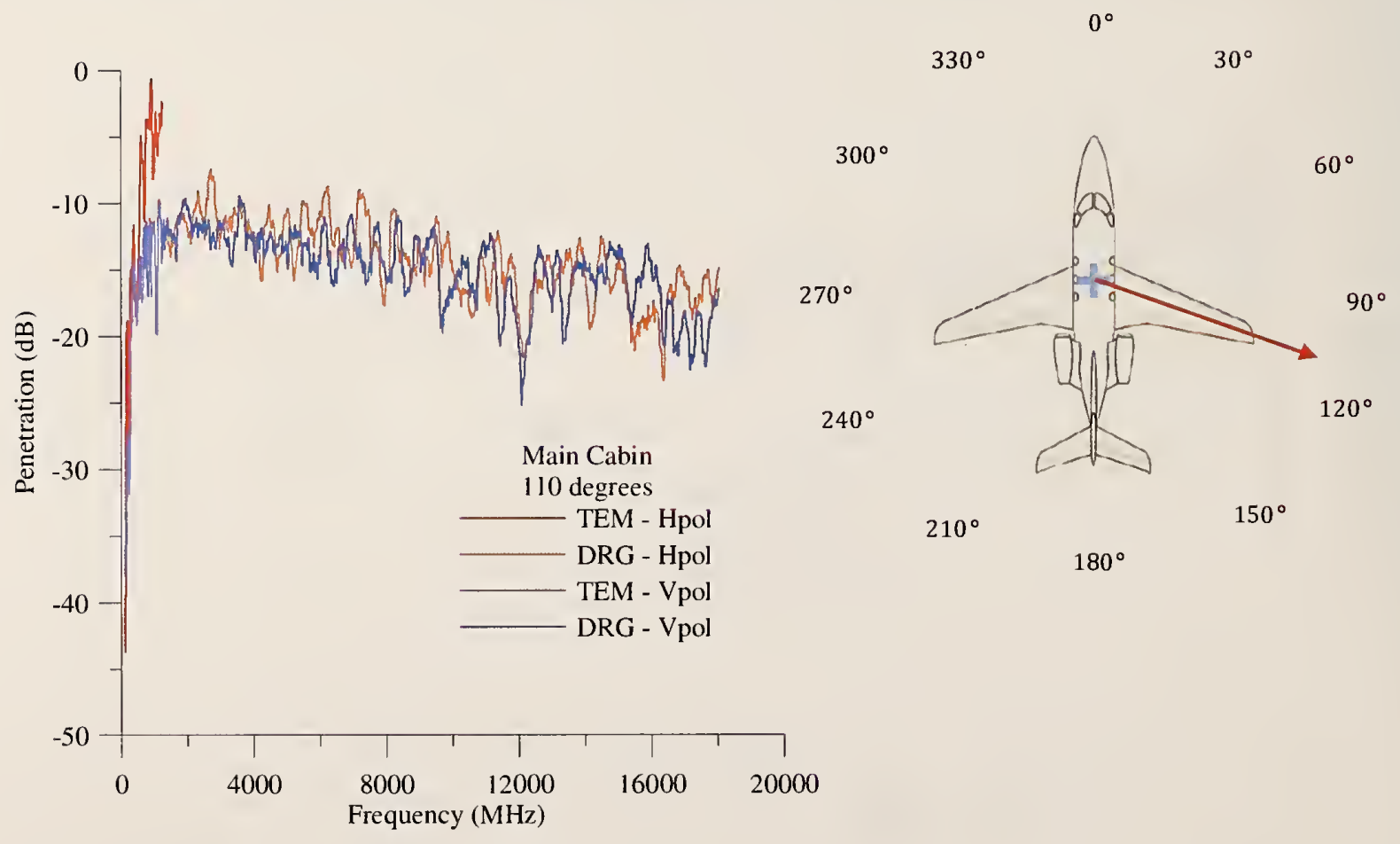

Figure 37. Penetration for all antennas and polarizations for the transmitting tower placed at the $110^{\circ}$ position and the receiving antennas placed in the main cabin. 


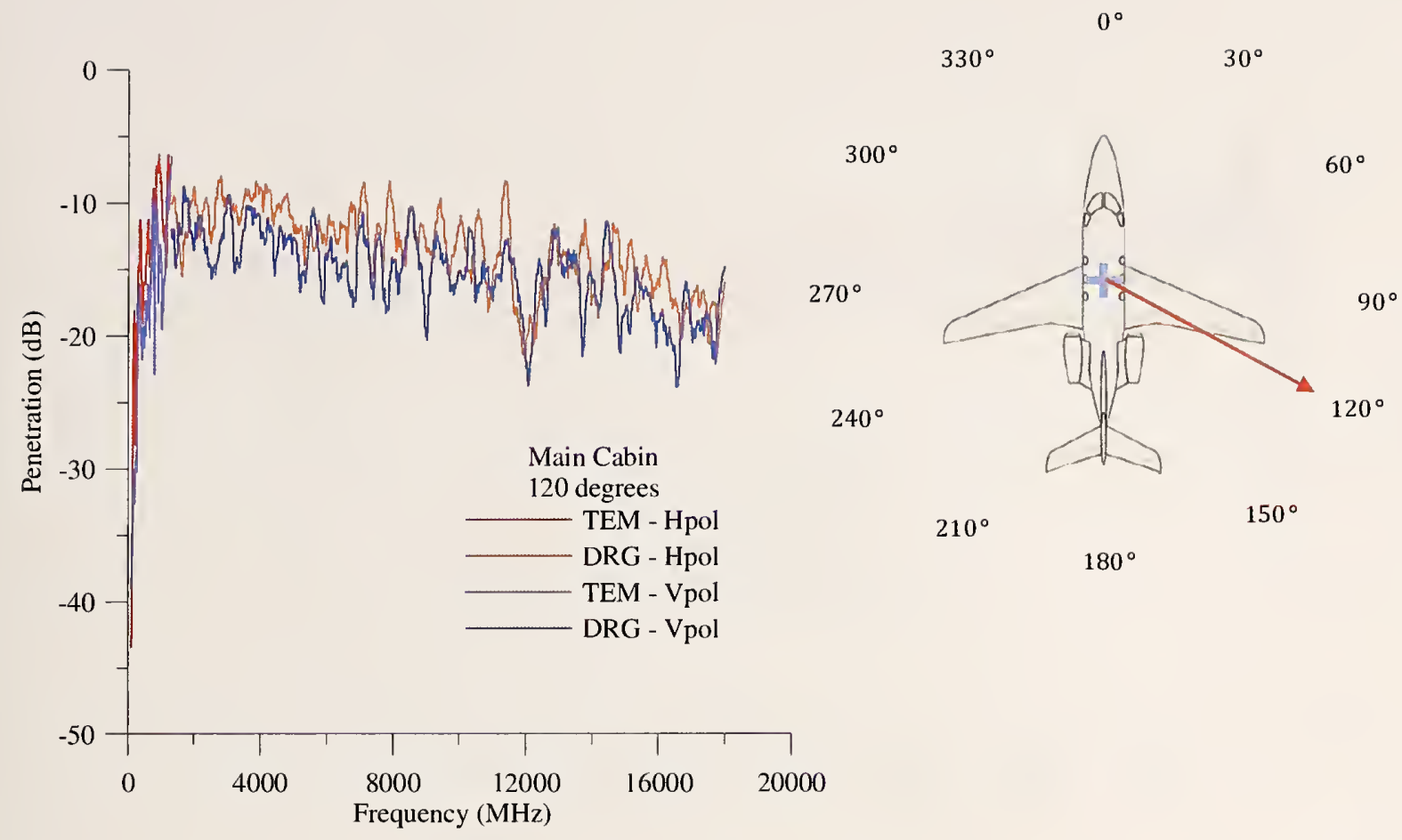

Figure 38. Penetration for all antennas and polarizations for the transmitting tower placed at the $120^{\circ}$ position and the receiving antennas placed in the main cabin.

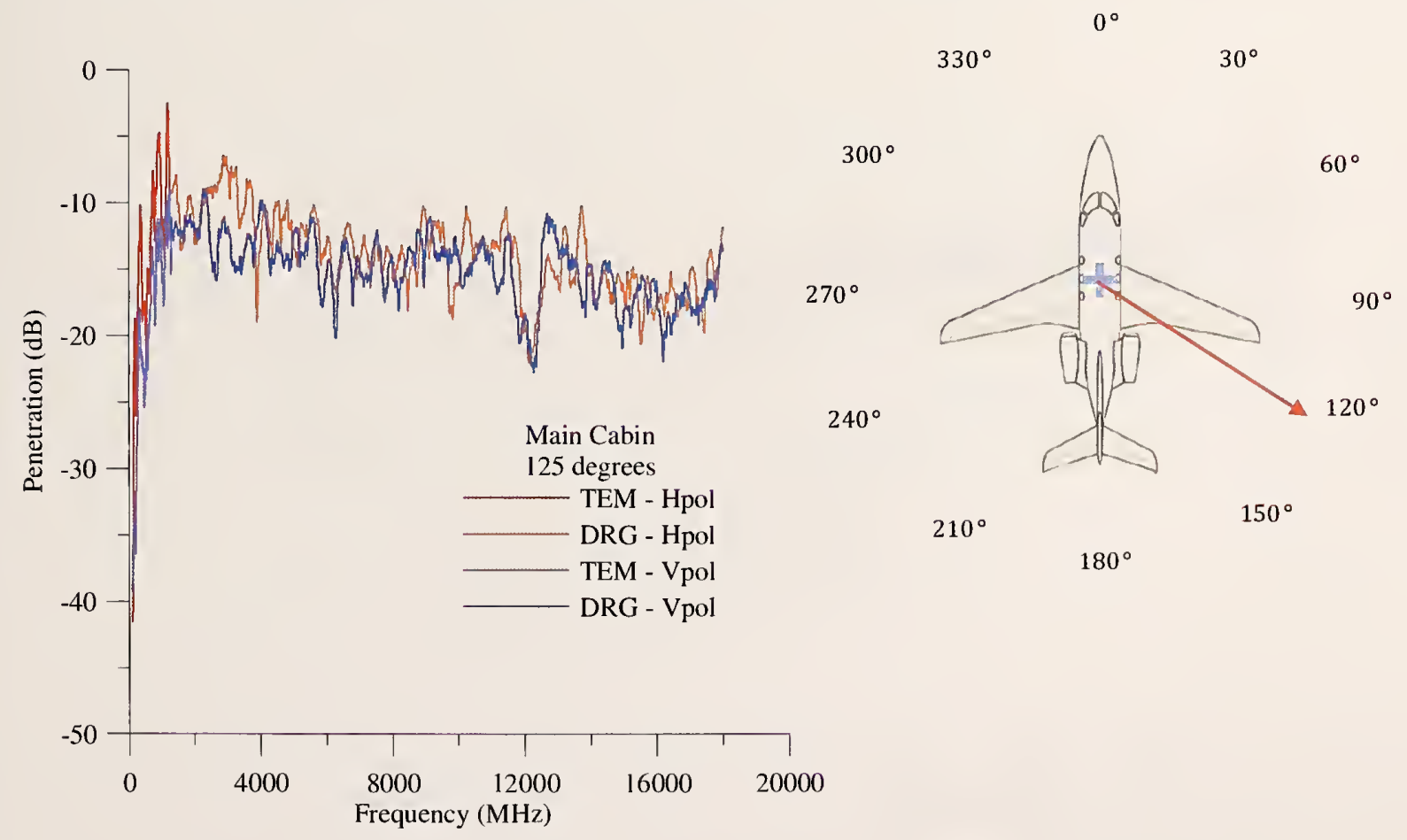

Figure 39. Penetration for all antennas and polarizations for the transmitting tower placed at the $125^{\circ}$ position and the receiving antennas placed in the main cabin. 


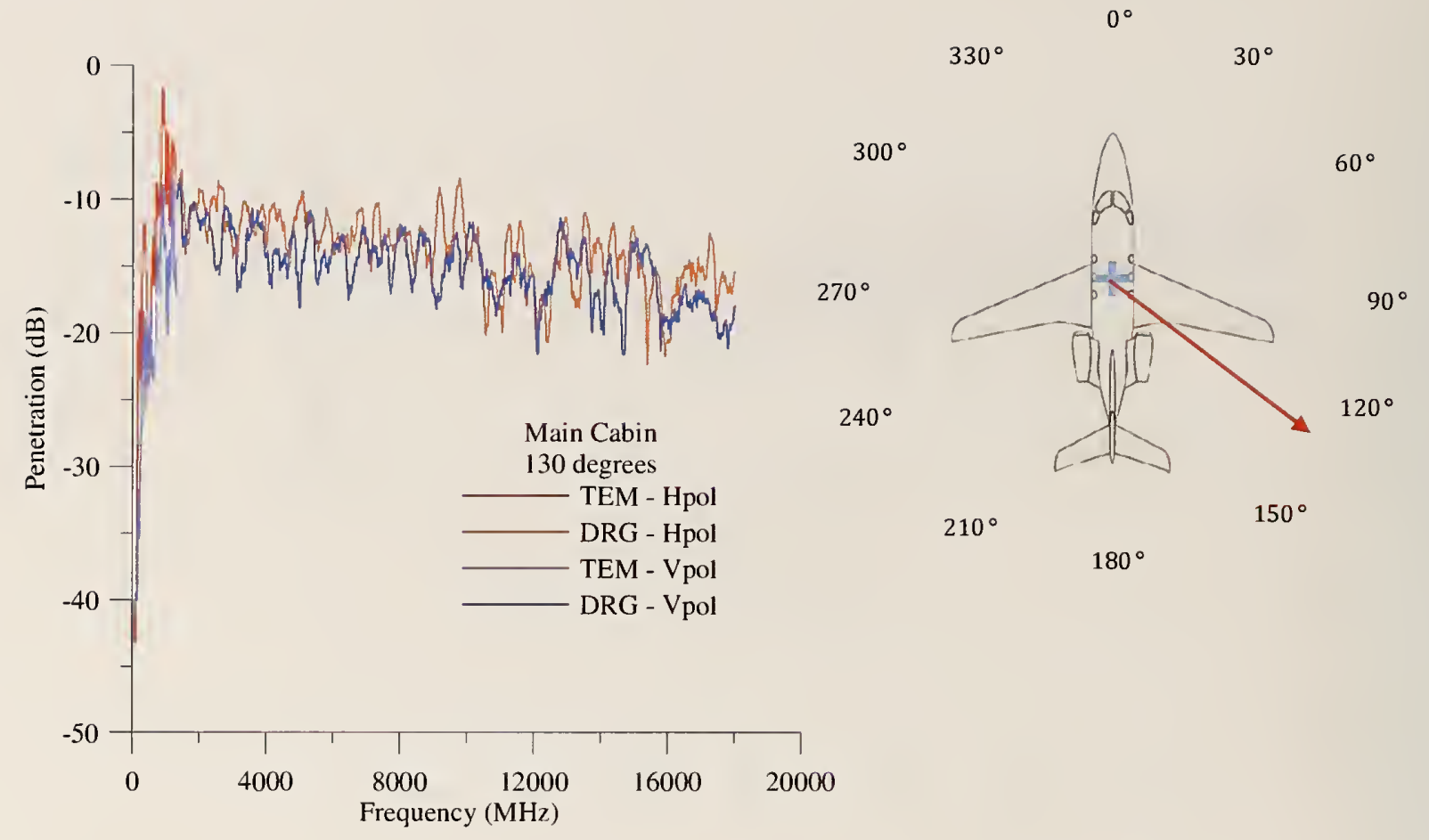

Figure 40. Penetration for all antennas and polarizations for the transmitting tower placed at the $130^{\circ}$ position and the receiving antennas placed in the main cabin.

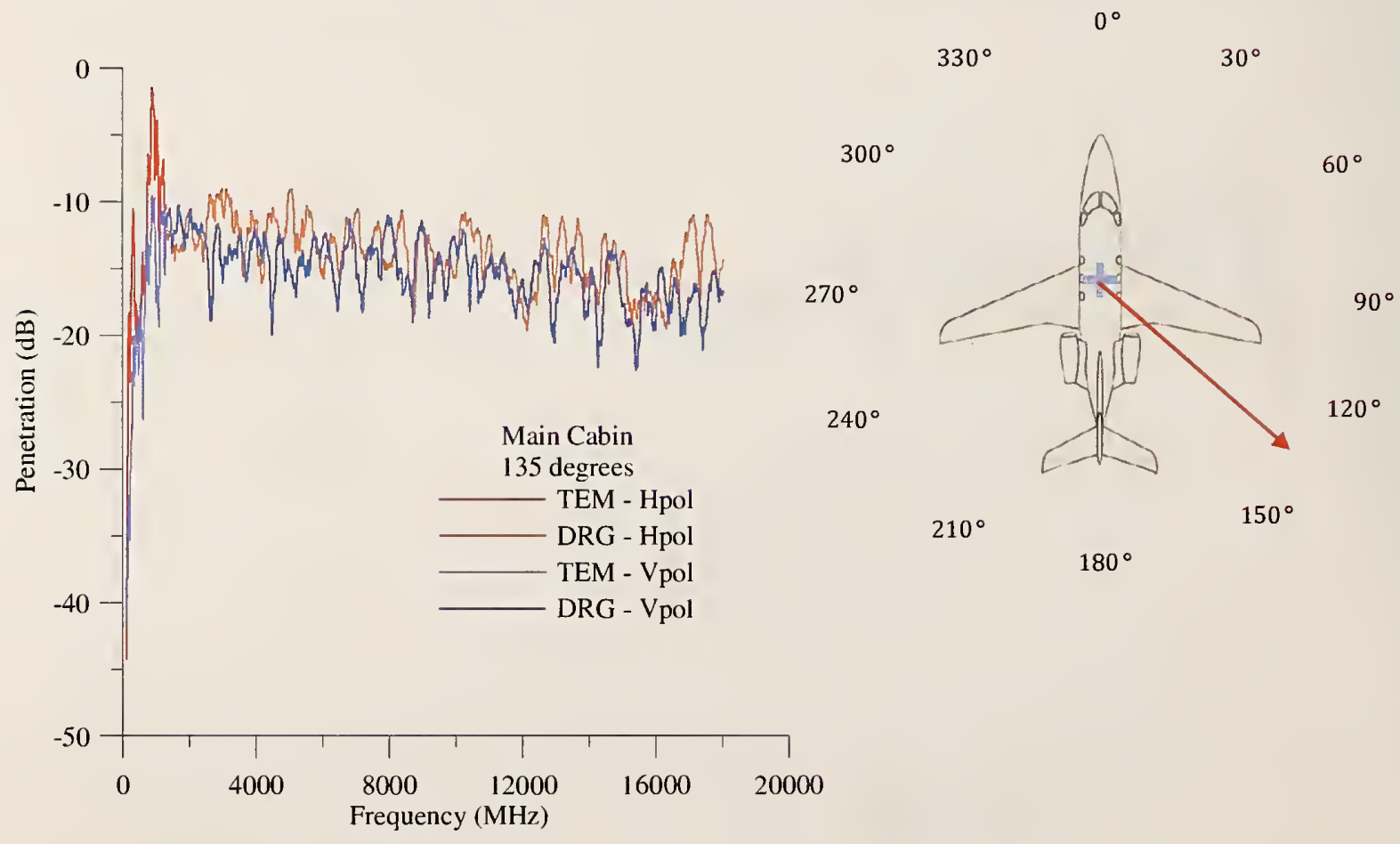

Figure 41. Penetration for all antennas and polarizations for the transmitting tower placed at the $135^{\circ}$ position and the receiving antennas placed in the main cabin. 


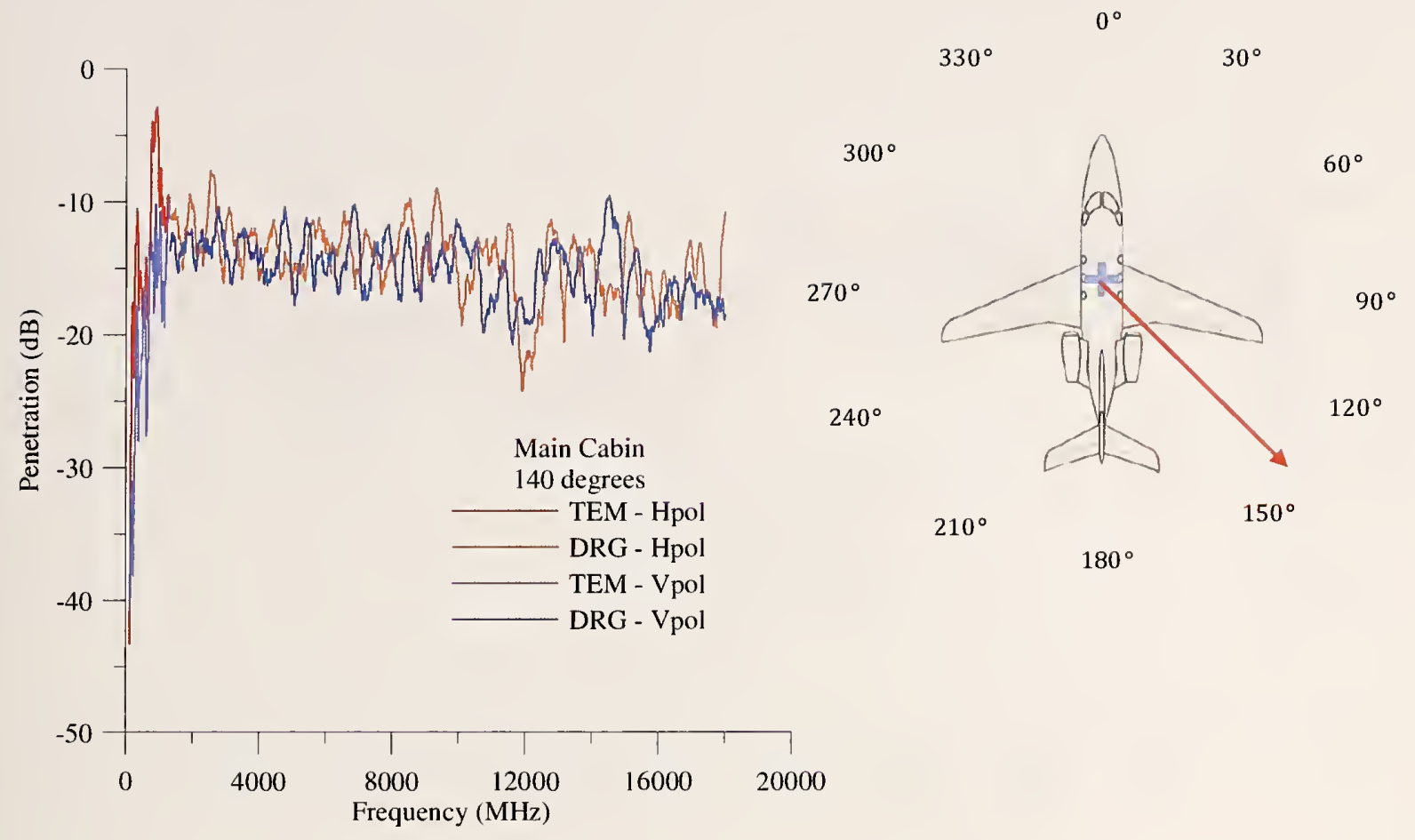

Figure 42. Penetration for all antennas and polarizations for the transmitting tower placed at the $140^{\circ}$ position and the receiving antennas placed in the main cabin.

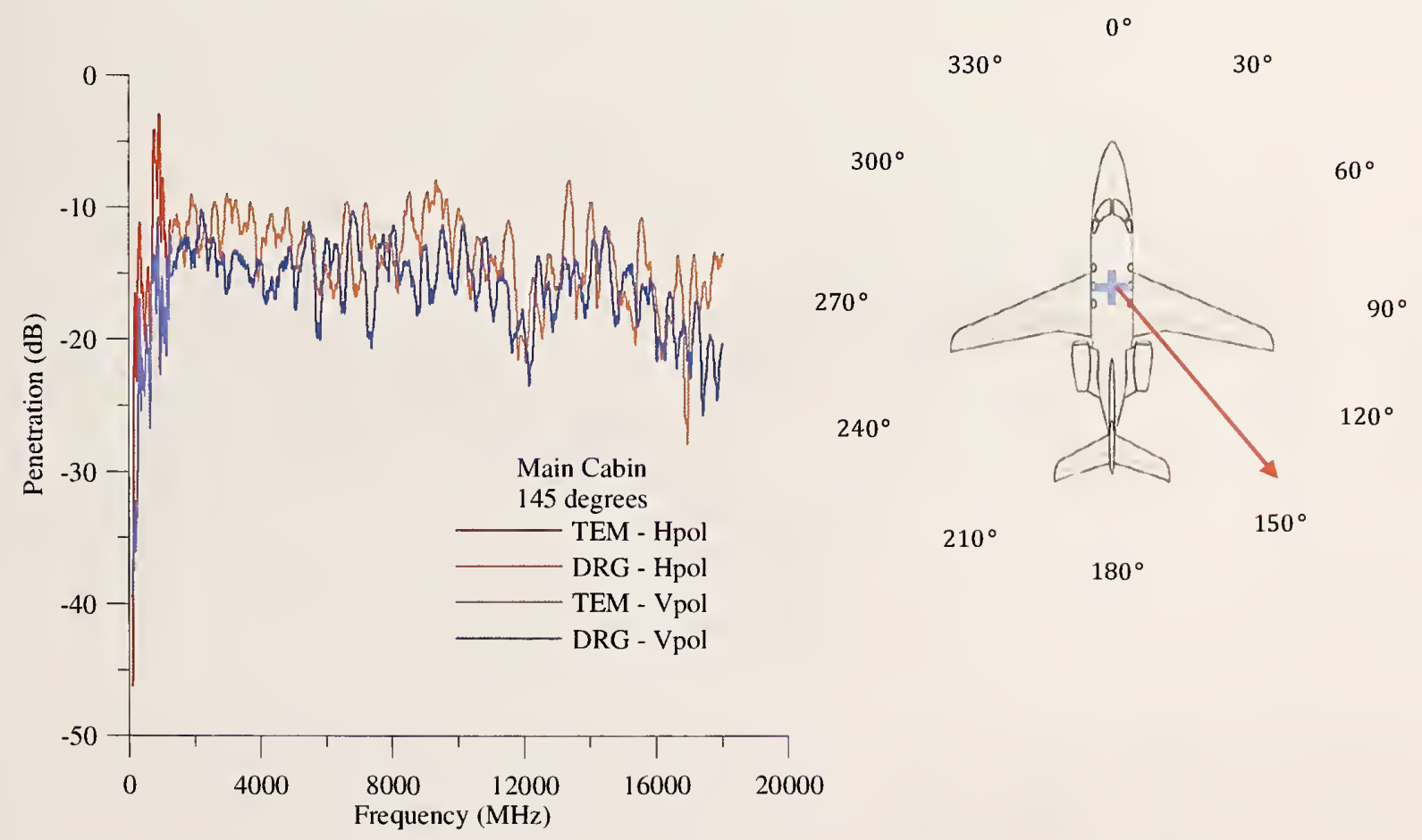

Figure 43. Penetration for all antennas and polarizations for the transmitting tower placed at the $145^{\circ}$ position and the receiving antennas placed in the main cabin. 


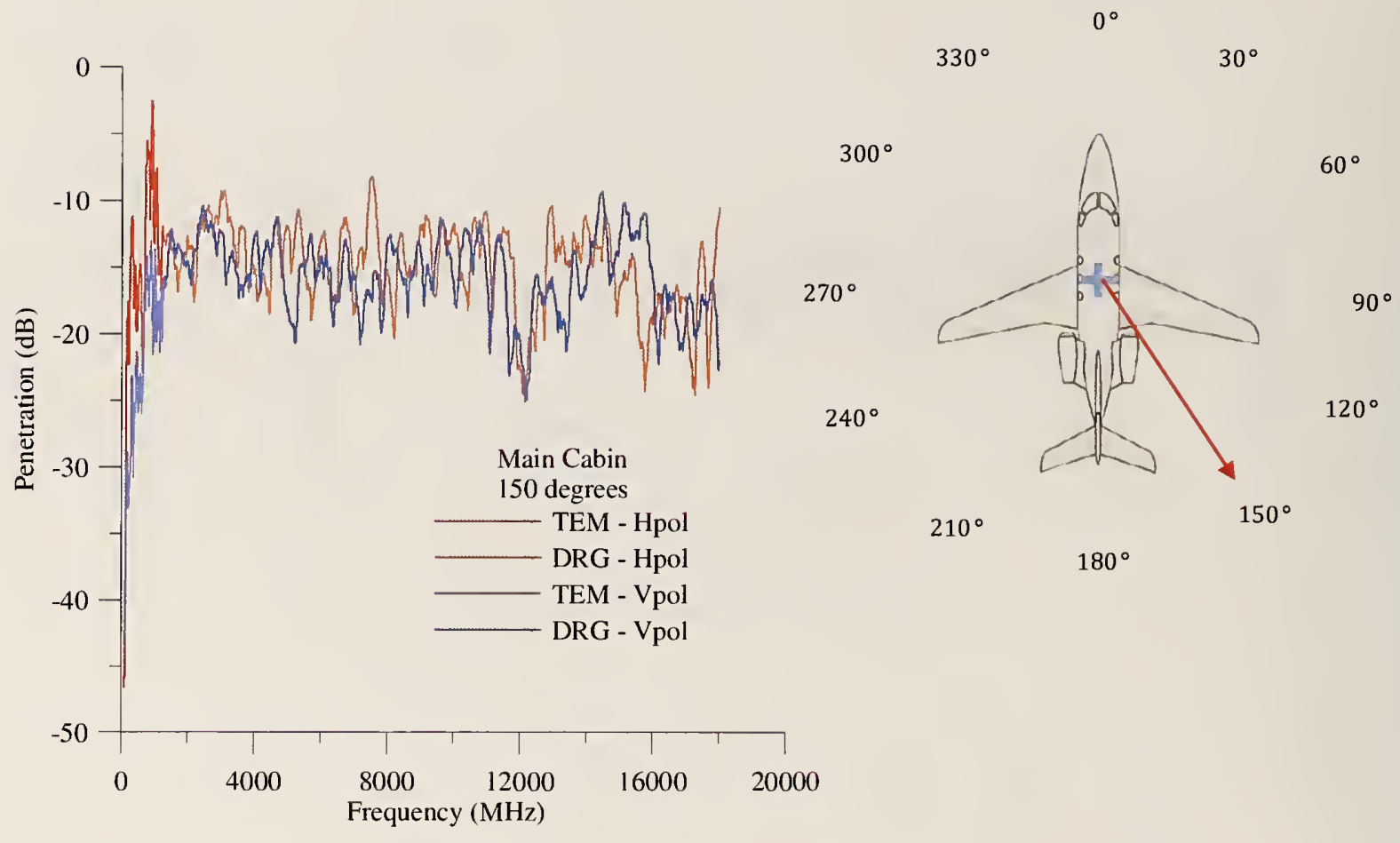

Figure 44. Penetration for all antennas and polarizations for the transmitting tower placed at the $150^{\circ}$ position and the receiving antennas placed in the main cabin.

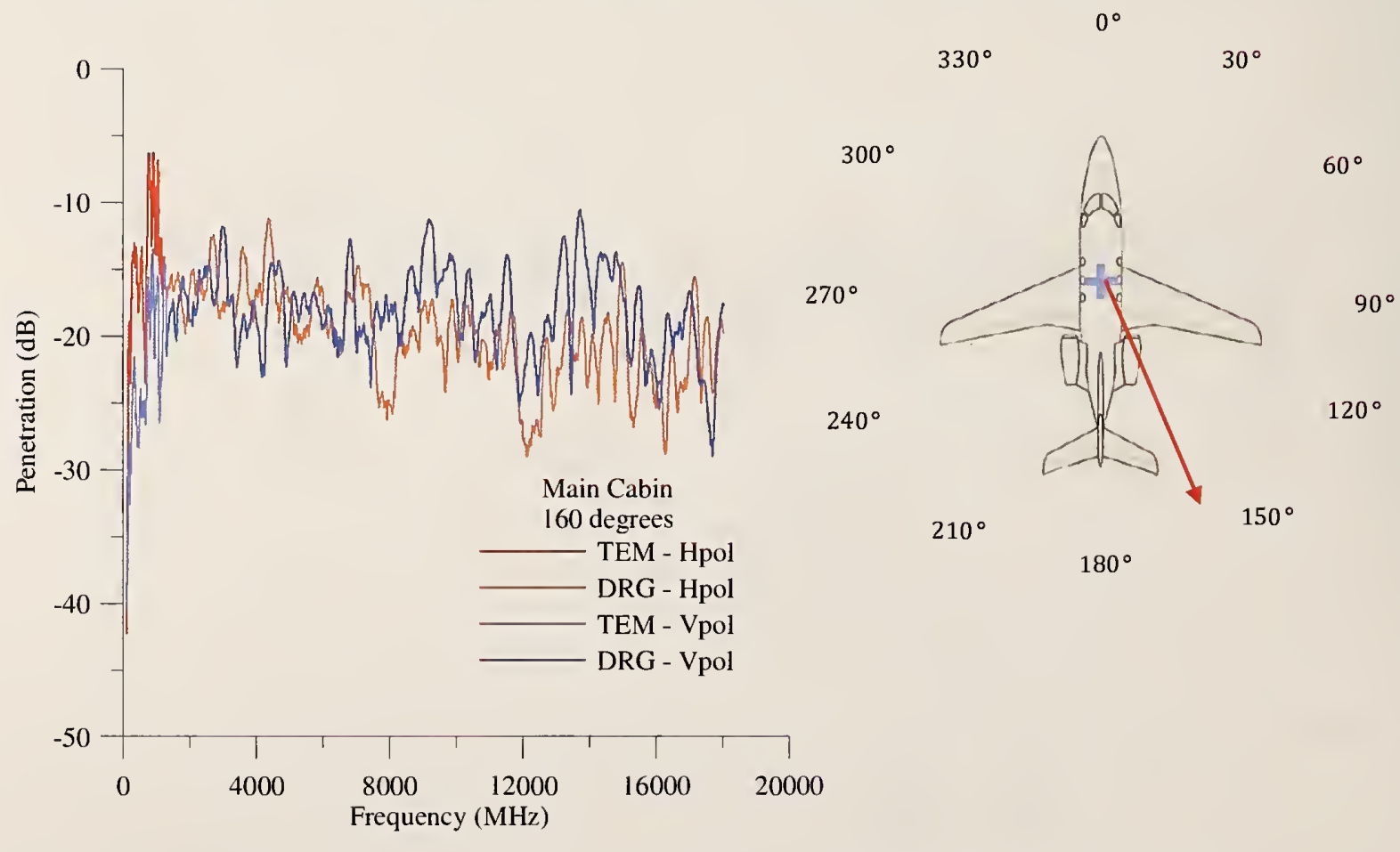

Figure 45. Penetration for all antennas and polarizations for the transmitting tower placed at the $160^{\circ}$ position and the receiving antennas placed in the main cabin. 


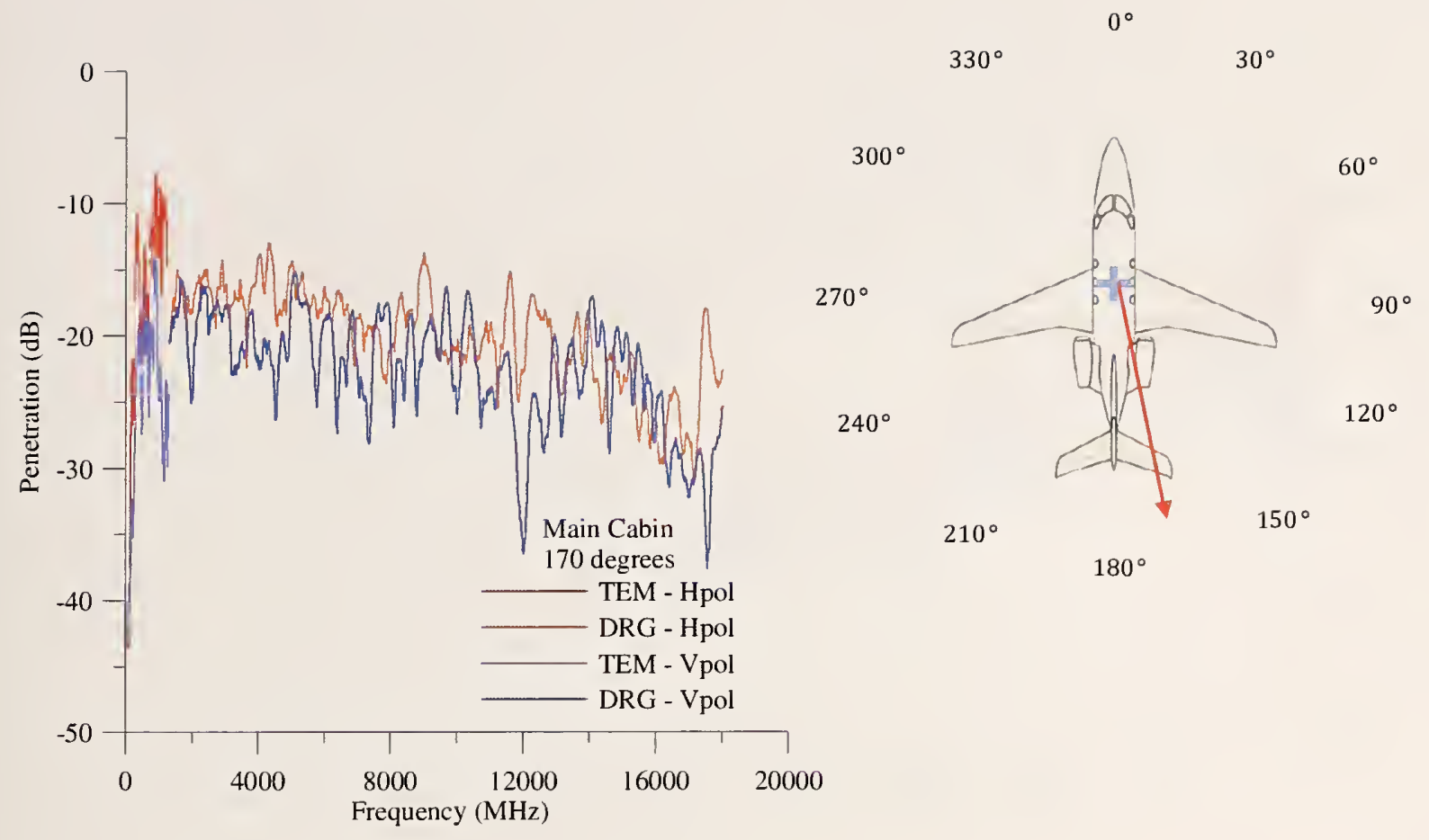

Figure 46. Penetration for all antennas and polarizations for the transmitting tower placed at the $170^{\circ}$ position and the receiving antennas placed in the main cabin.

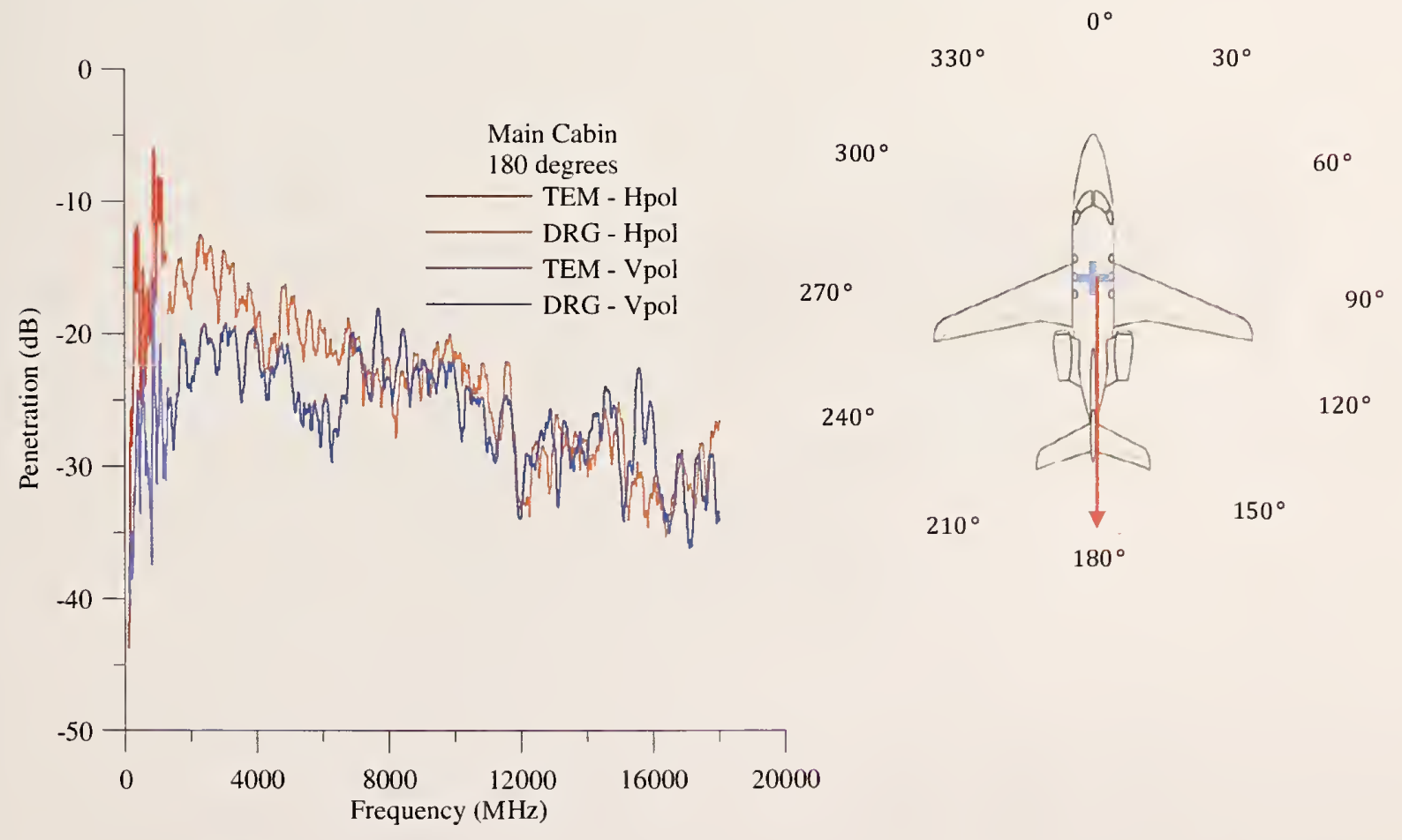

Figure 47. Penetration for all antennas and polarizations for the transmitting tower placed at the $180^{\circ}$ position and the receiving antennas placed in the main cabin. 


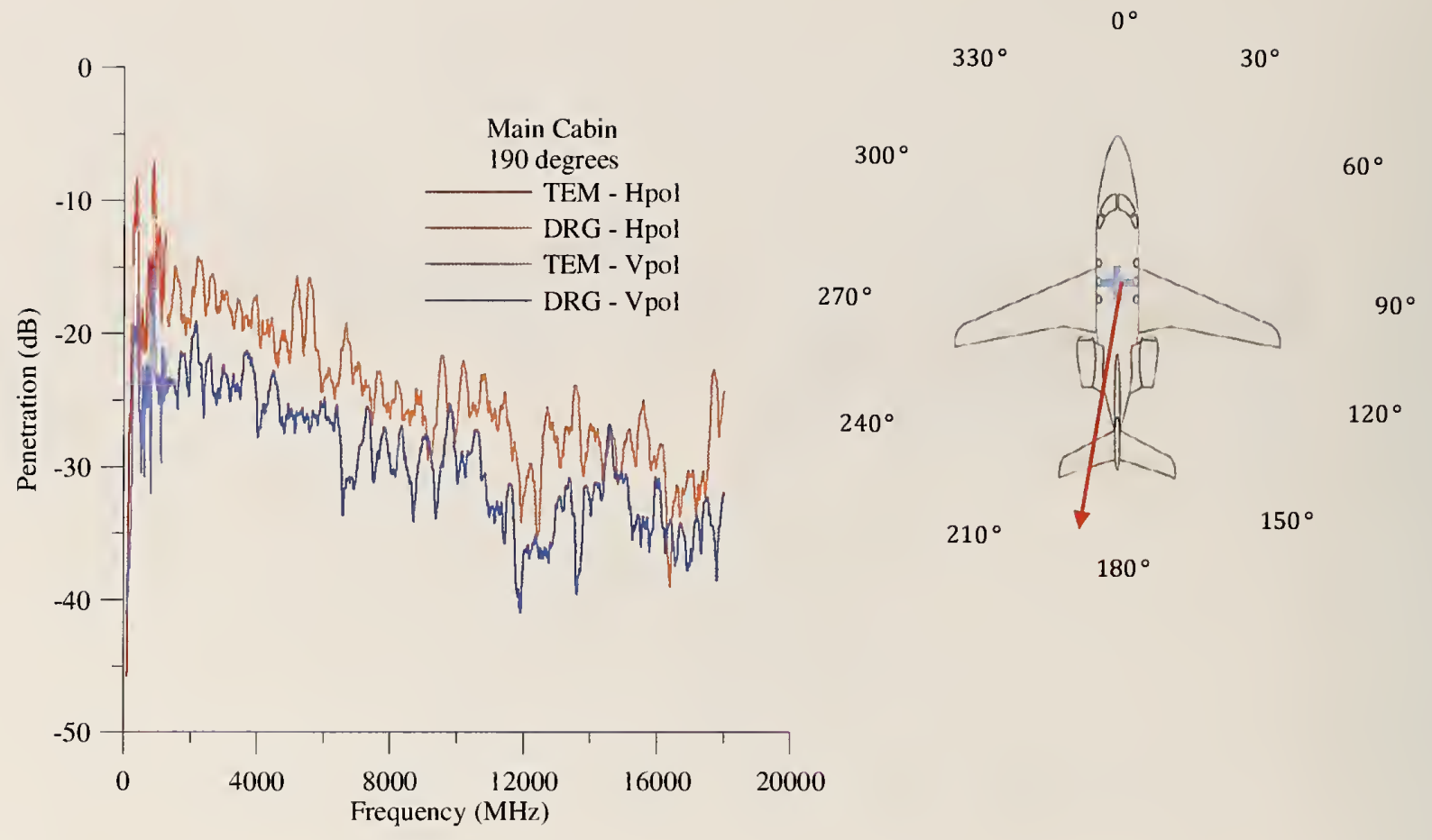

Figure 48. Penetration for all antennas and polarizations for the transmitting tower placed at the $190^{\circ}$ position and the receiving antennas placed in the main cabin.

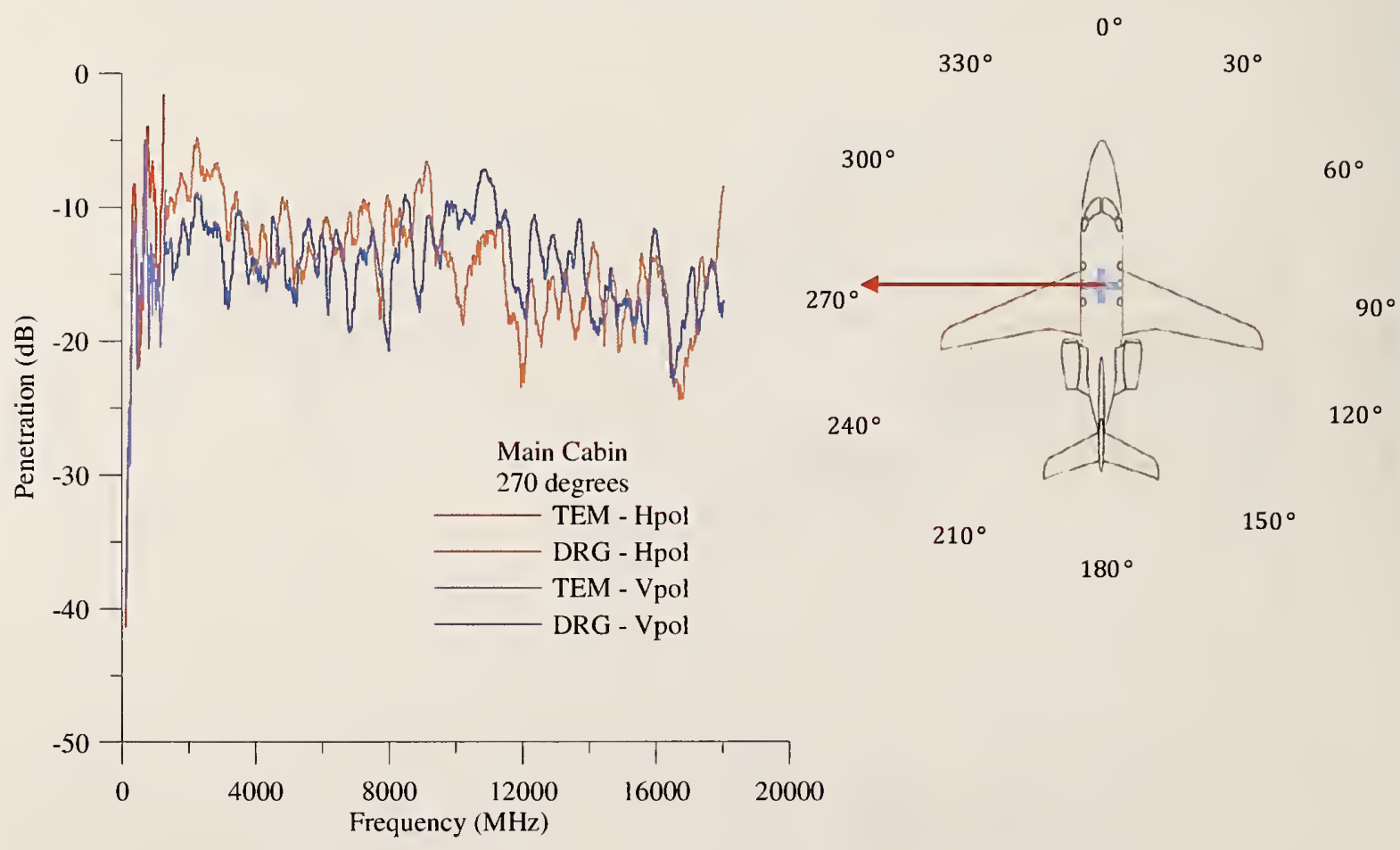

Figure 49. Penetration for all antennas and polarizations for the transmitting tower placed at the $270^{\circ}$ position and the receiving antennas placed in the main cabin. 


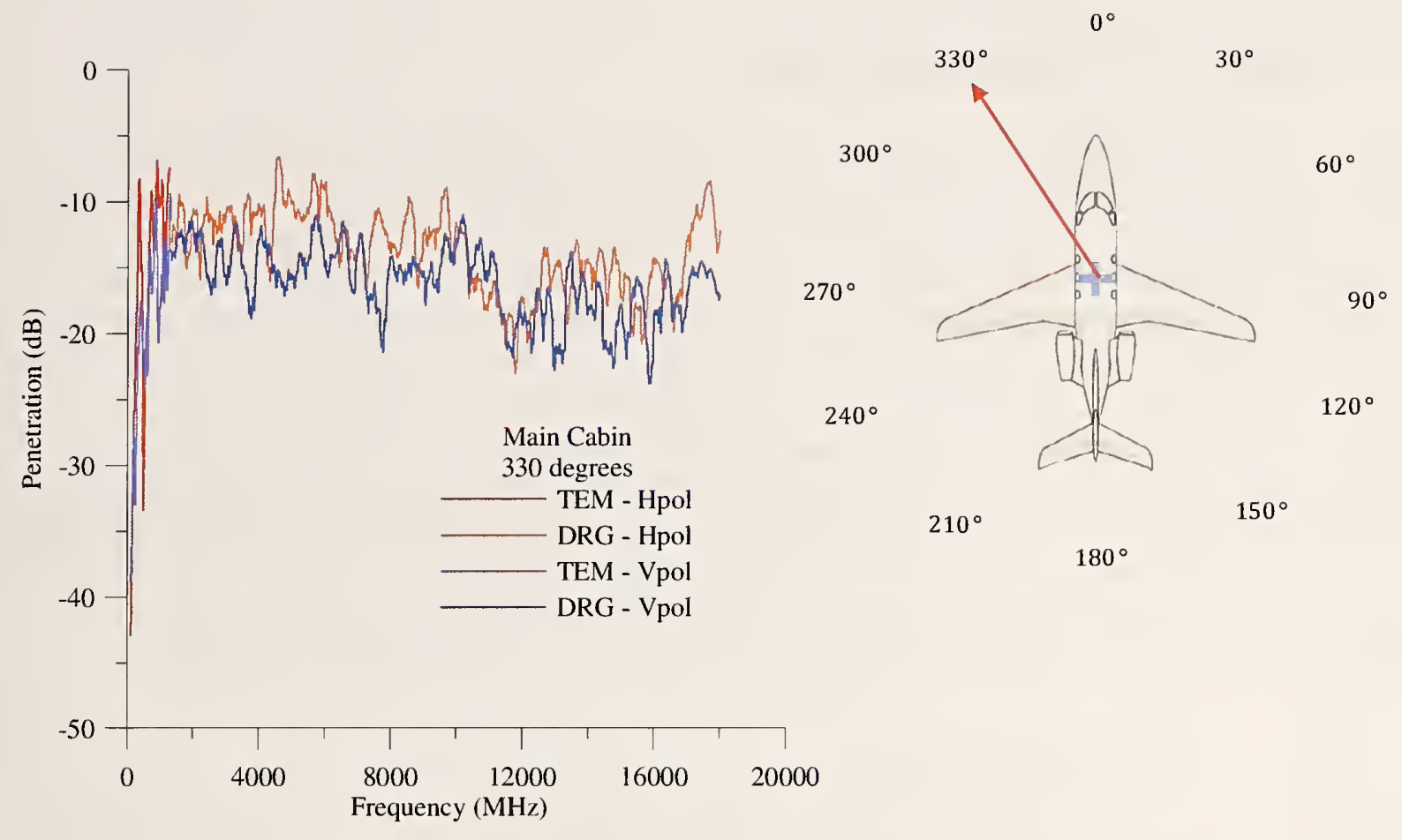

Figure 50. Penetration for all antennas and polarizations for the transmitting tower placed at the $330^{\circ}$ position and the receiving antennas placed in the main cabin. 


\subsection{Flight Deck Penetration Results}

The second penetration study was performed with the receiving antennas placed in the flight deck between the pilot's and the co-pilot's seat, as shown in Figure 51. The time-domain plots for the DRG antennas in the horizontal polarization are shown in Figure 52 for various angular positions around the aircraft. There are direct coupling components visible for almost every position around the aircraft. Figure 53 shows various penetration positions around the aircraft. Maximum penetration of approximately $0 \mathrm{~dB}$ occurs at the nose position for frequencies around $650 \mathrm{MHz}, 770 \mathrm{MHz}, 1000 \mathrm{MHz}, 1525 \mathrm{MHz}$, and $3100 \mathrm{MHz}$. It then drops to $-20 \mathrm{~dB}$ at frequencies above $2000 \mathrm{MHz}$. Minimum penetration occurs at the $180^{\circ}$ position. For frequencies below $2000 \mathrm{MHz}$, horizontal polarization has penetration values around $-20 \mathrm{~dB}$, and for vertical polarization, penetration values are around $-25 \mathrm{~dB}$. Penetration values of $-40 \mathrm{~dB}$ for the vertical polarization and $-30 \mathrm{~dB}$ for the horizontal polarization are shown at frequencies close to $18,000 \mathrm{MHz}$. Figures 54 to 76 show each individual external position around the aircraft. Penetration decreases from the front to the back of the aircraft by about $20 \mathrm{~dB}$.

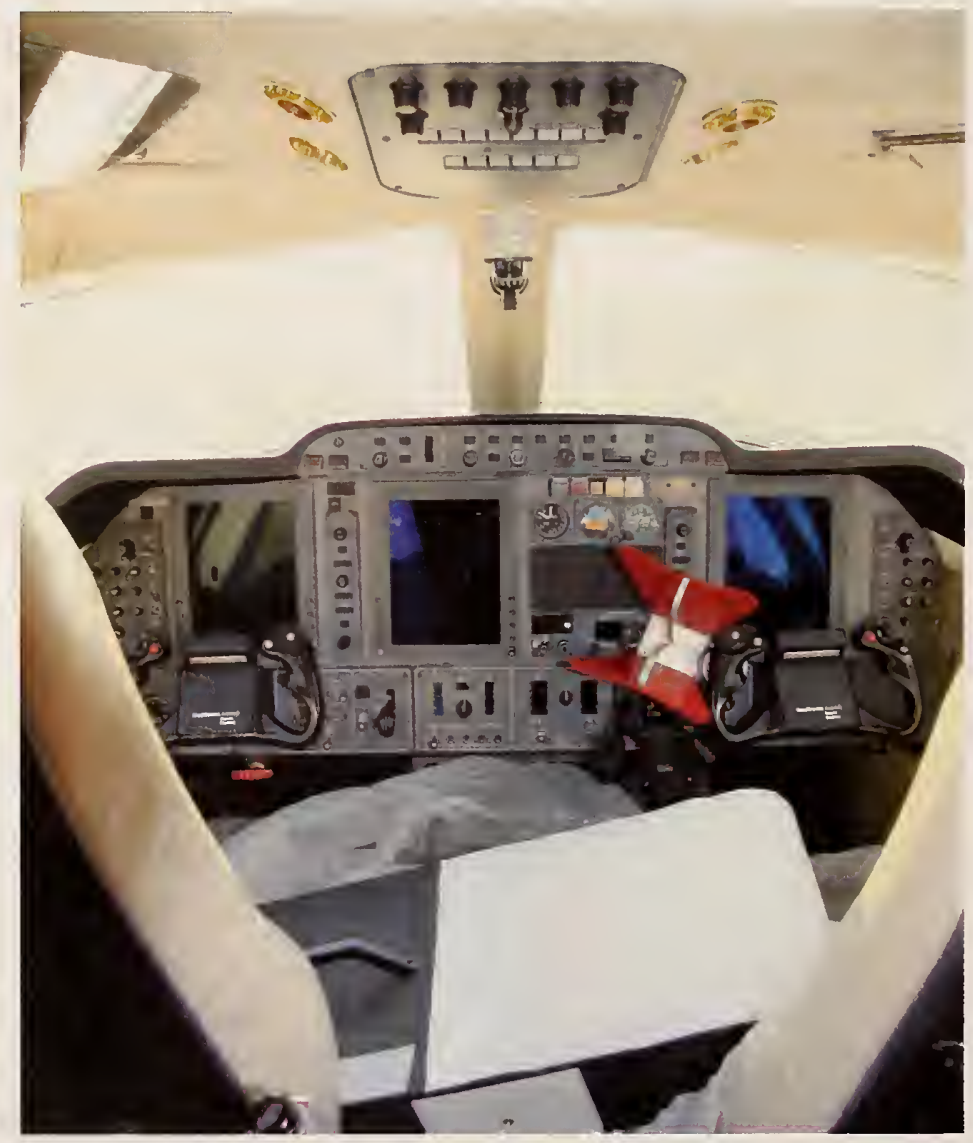

Figure 51. The receiving DRG and TEM antennas placed between the pilot's and co-pilot's seat. 


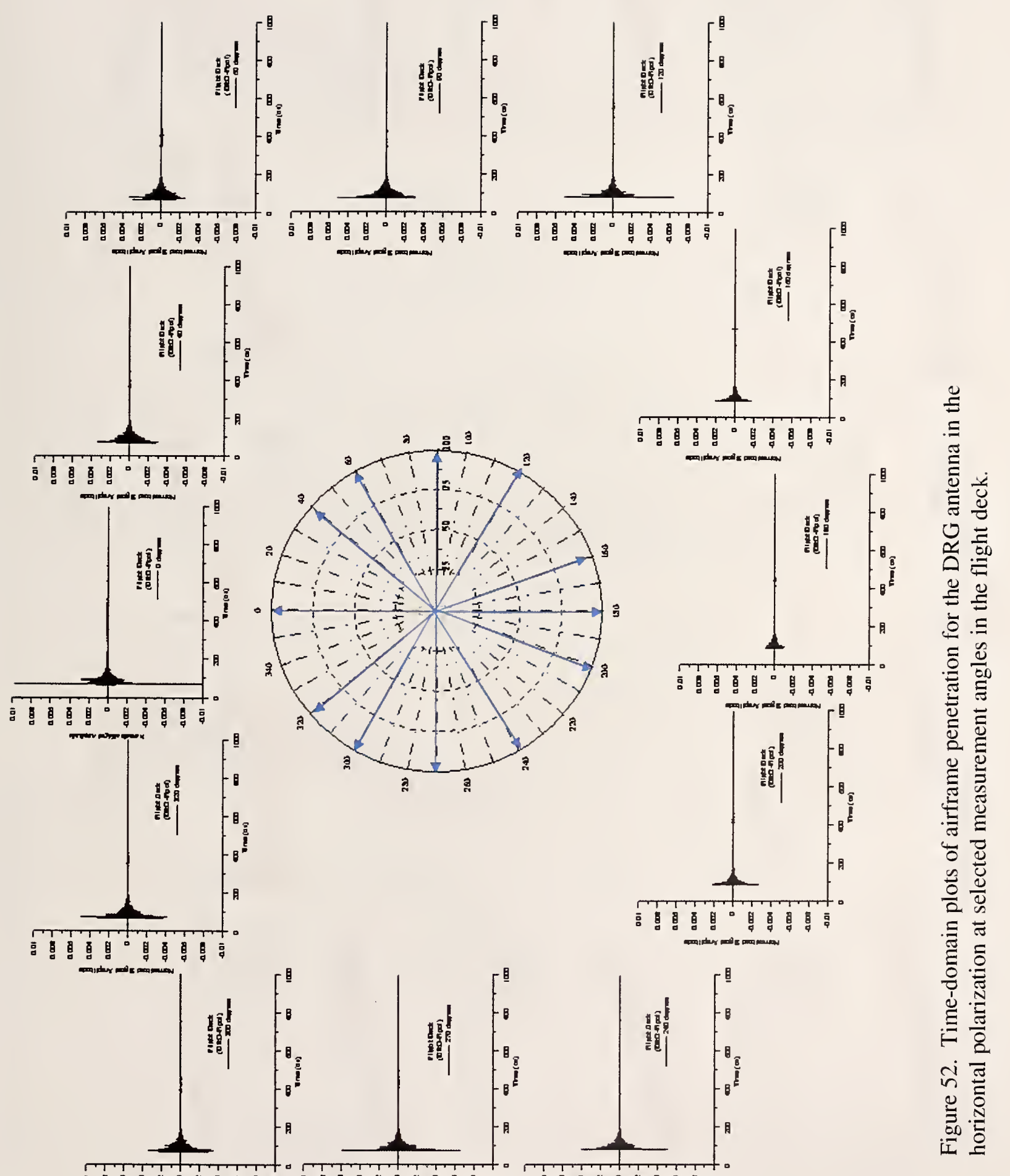




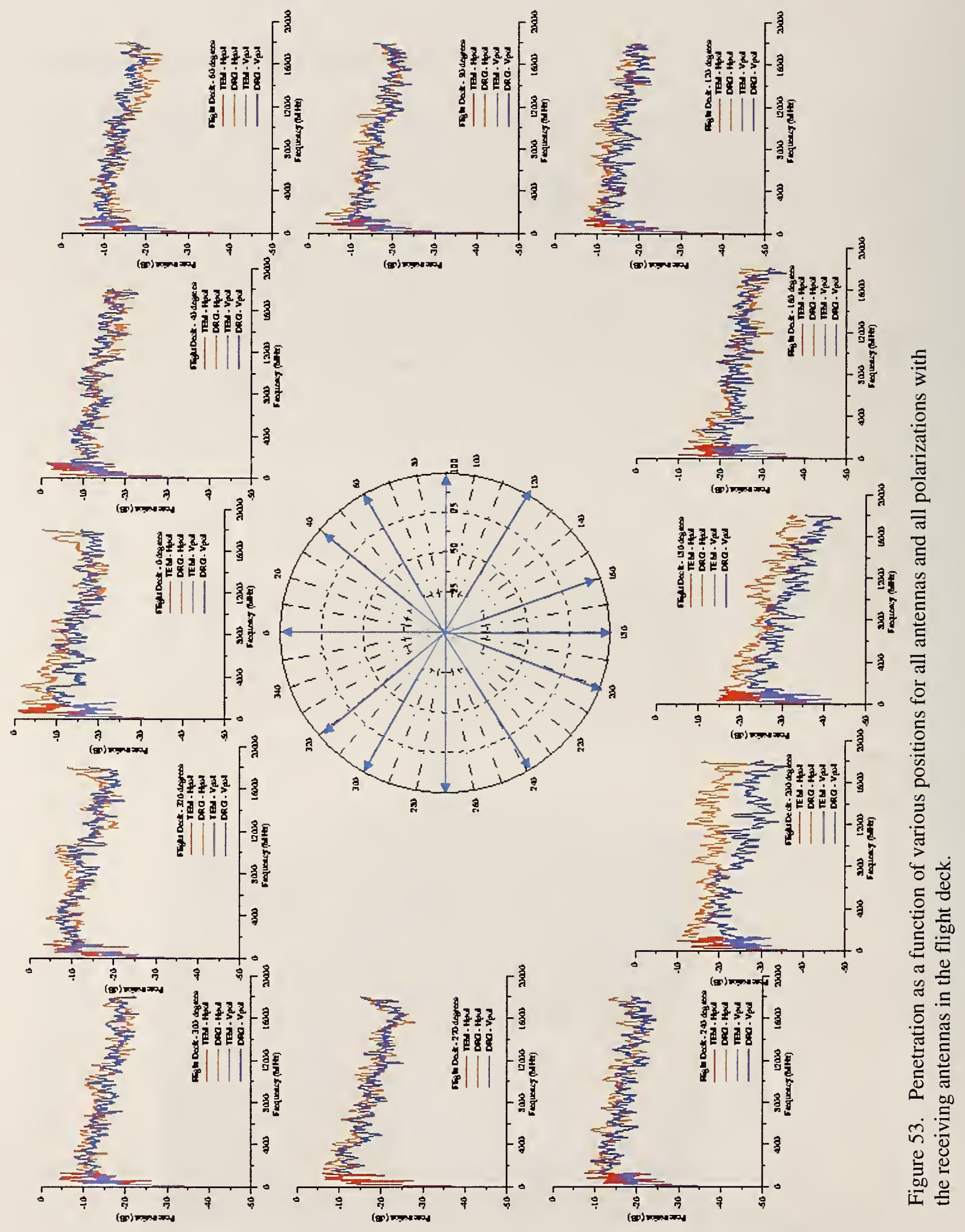



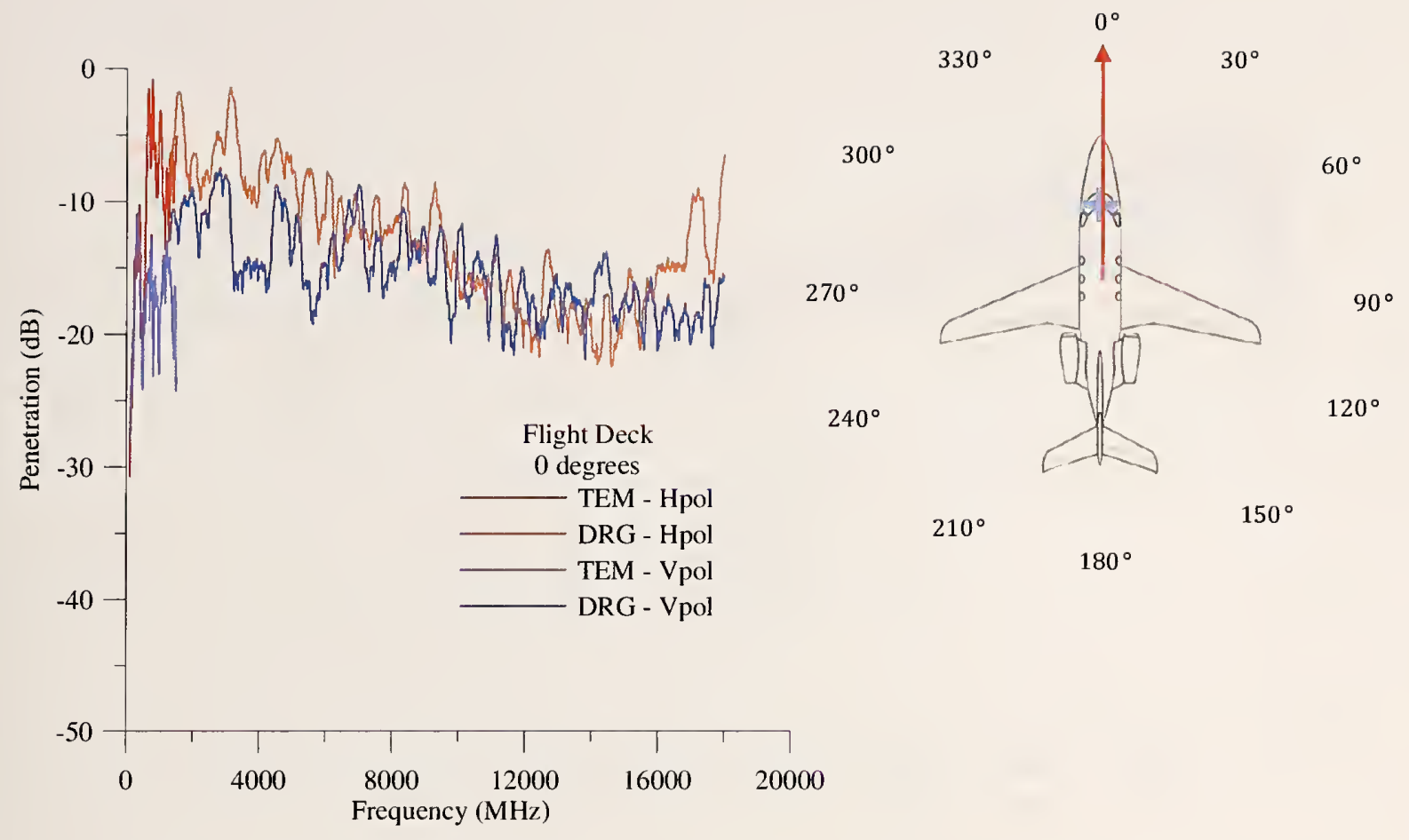

Figure 54. Penetration for all antennas and polarizations for the transmitting tower placed at the $0^{\circ}$ position and the receiving antennas placed in the flight deck.

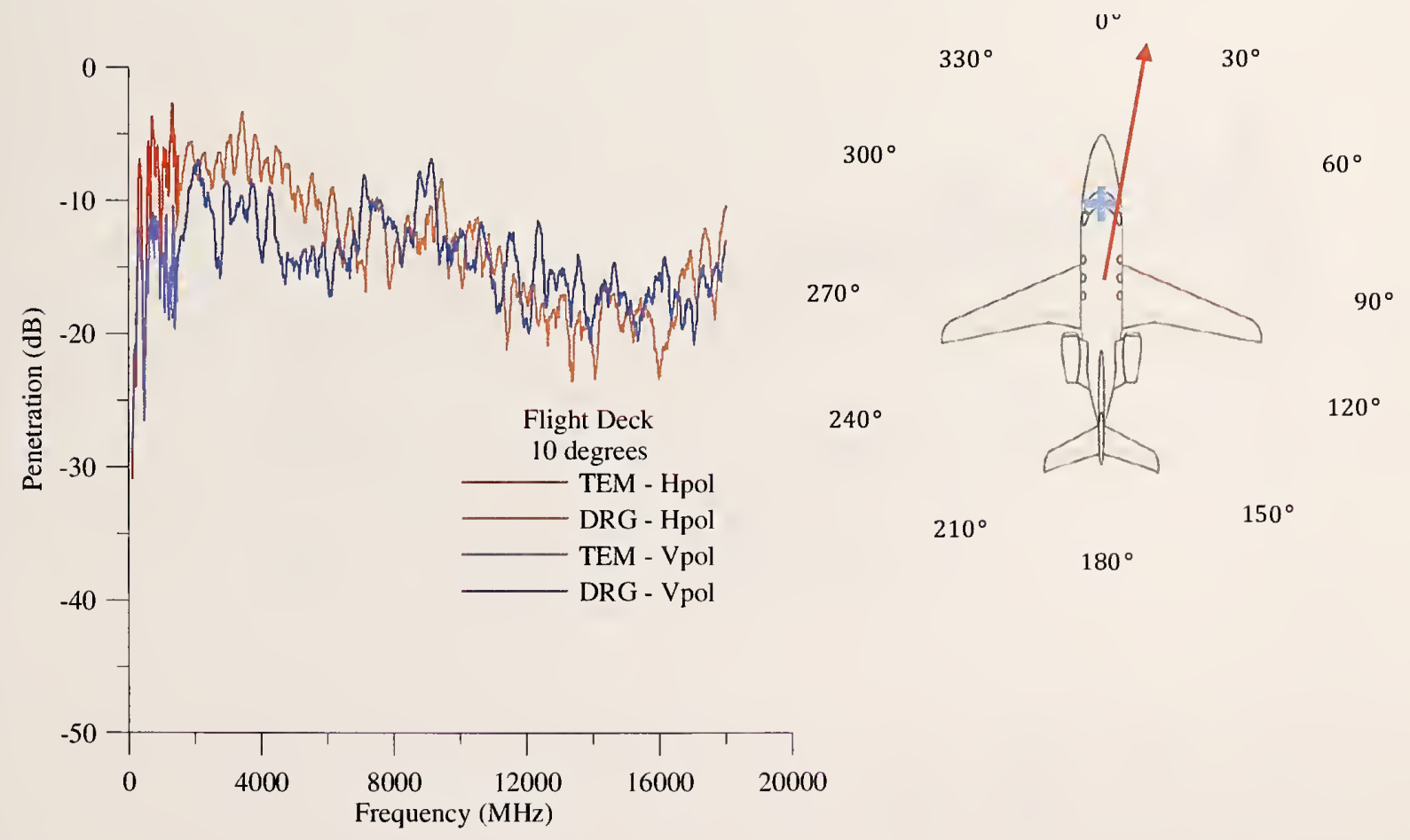

Figure 55. Penetration for all antennas and polarizations for the transmitting tower placed at the $10^{\circ}$ position and the receiving antennas placed in the flight deck. 


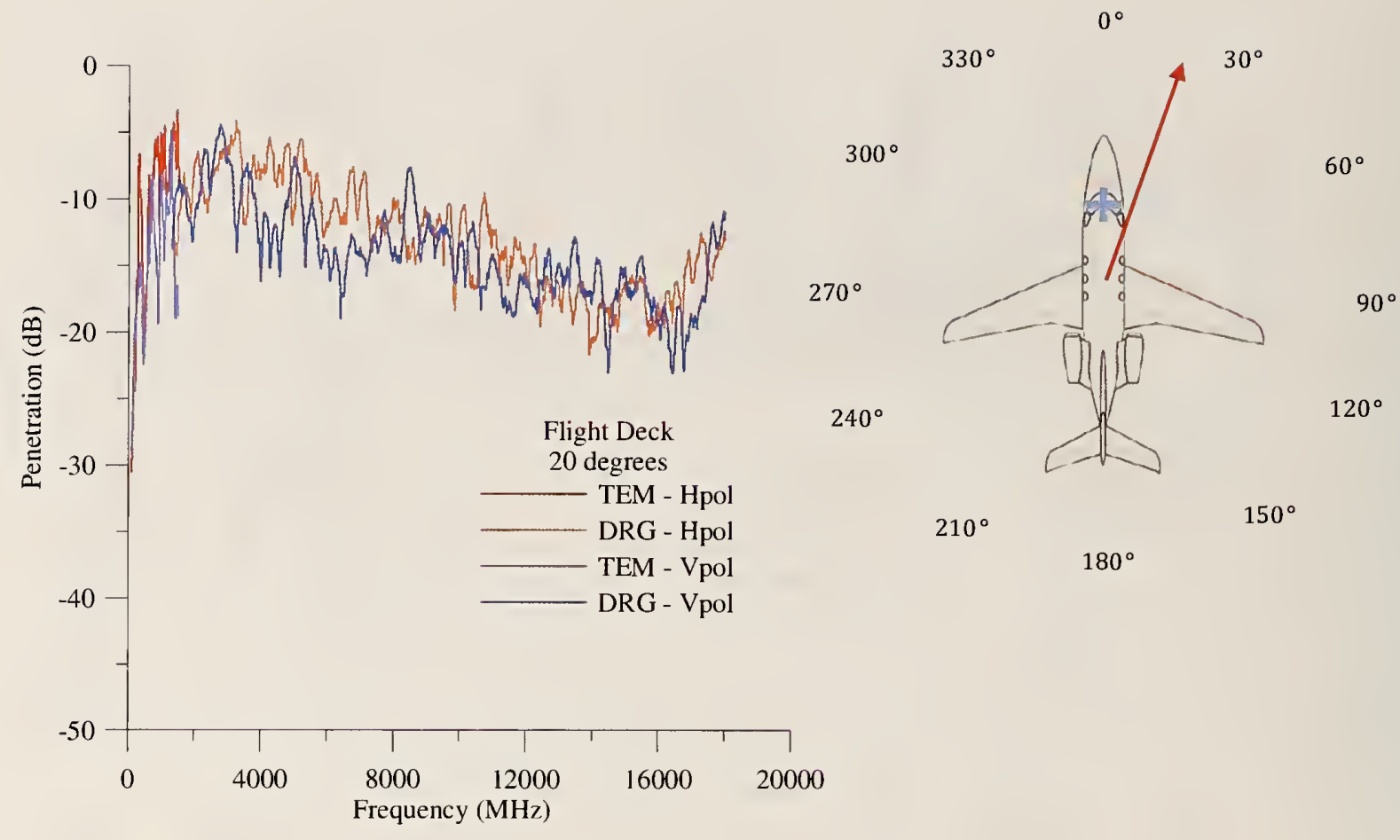

Figure 56. Penetration for all antennas and polarizations for the transmitting tower placed at the $20^{\circ}$ position and the receiving antennas placed in the flight deck.

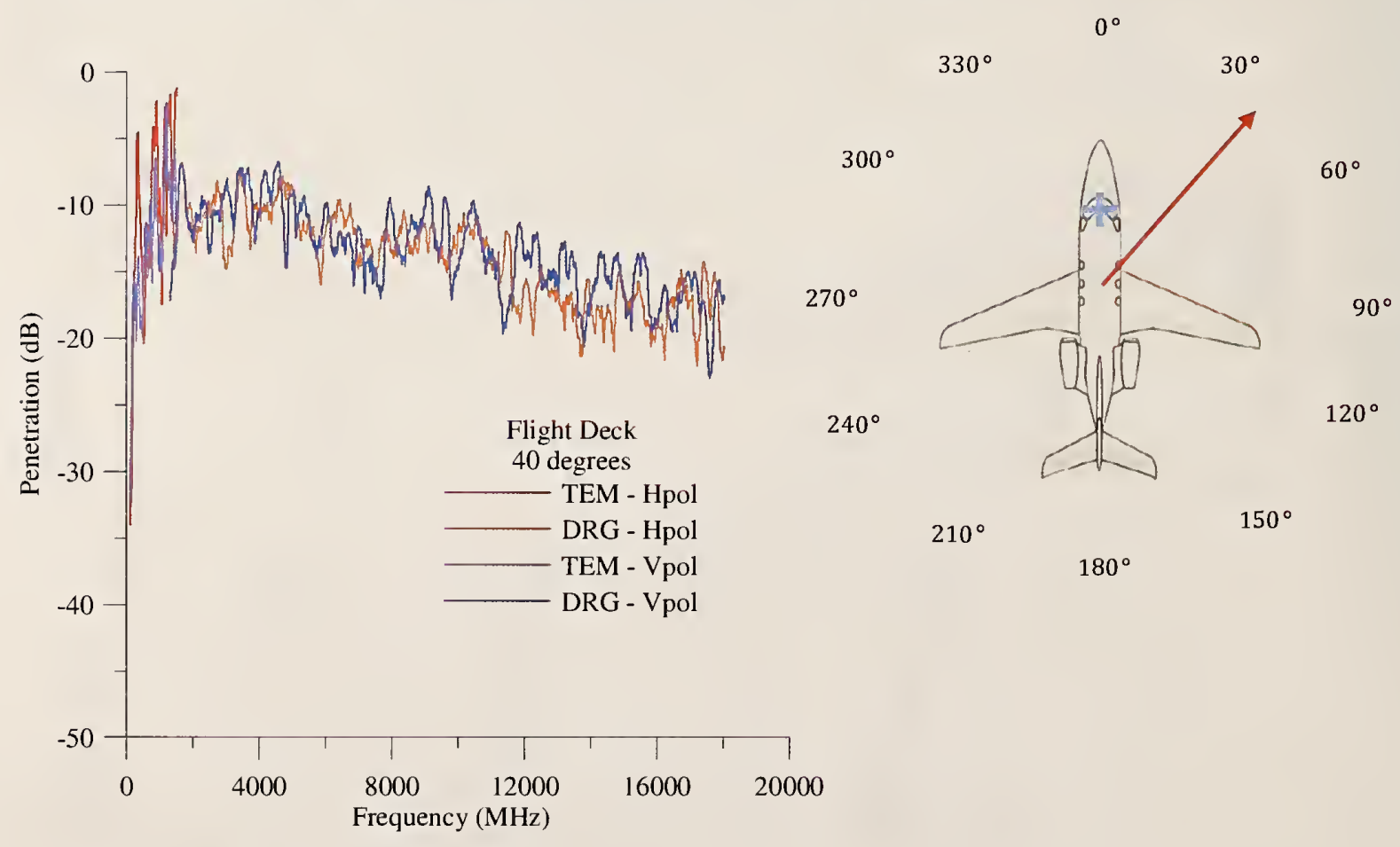

Figure 57. Penetration for all antennas and polarizations for the transmitting tower placed at the $40^{\circ}$ position and the receiving antennas placed in the flight deck. 


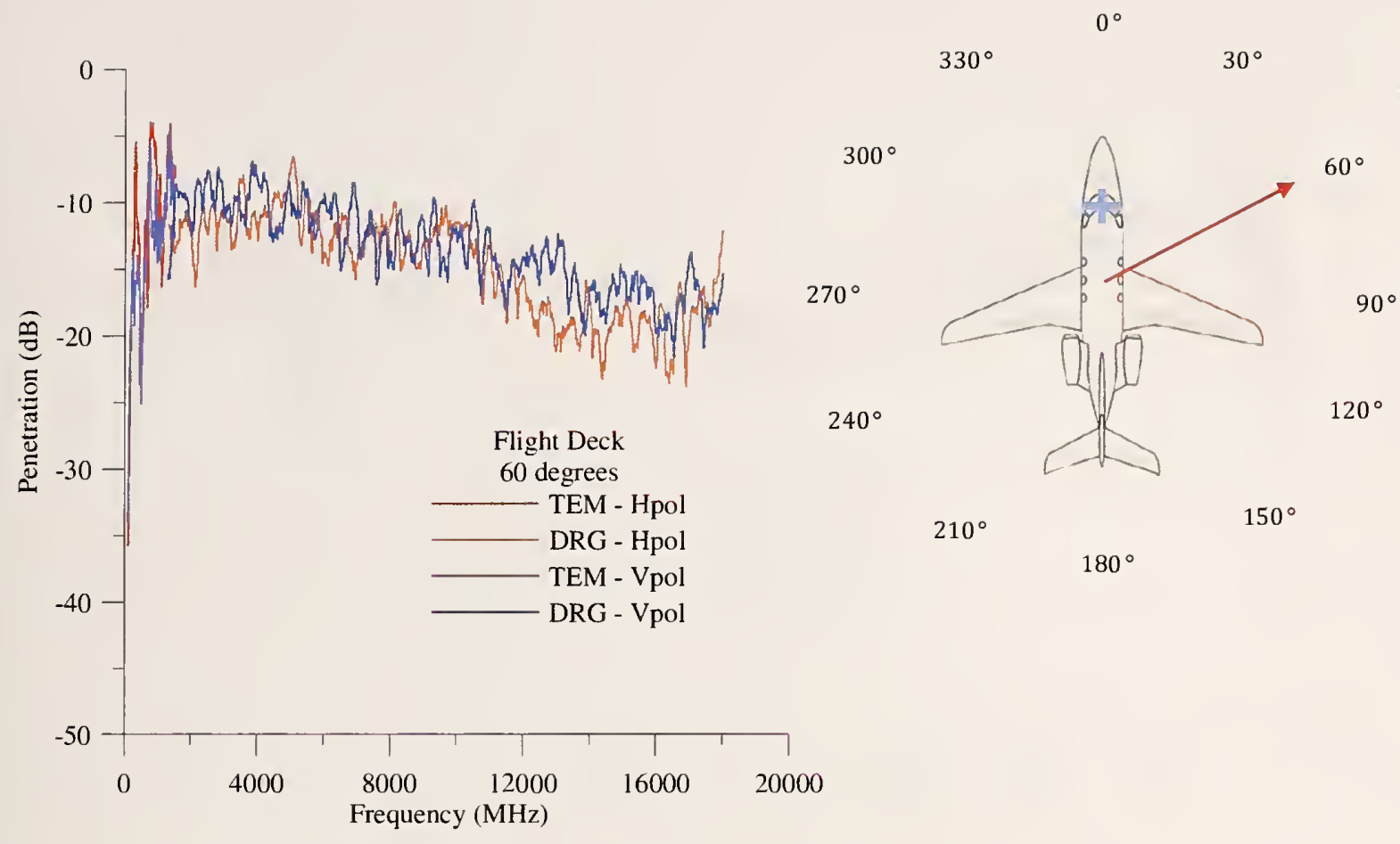

Figure 58. Penetration for all antennas and polarizations for the transmitting tower placed at the $60^{\circ}$ position and the receiving antennas placed in the flight deck.

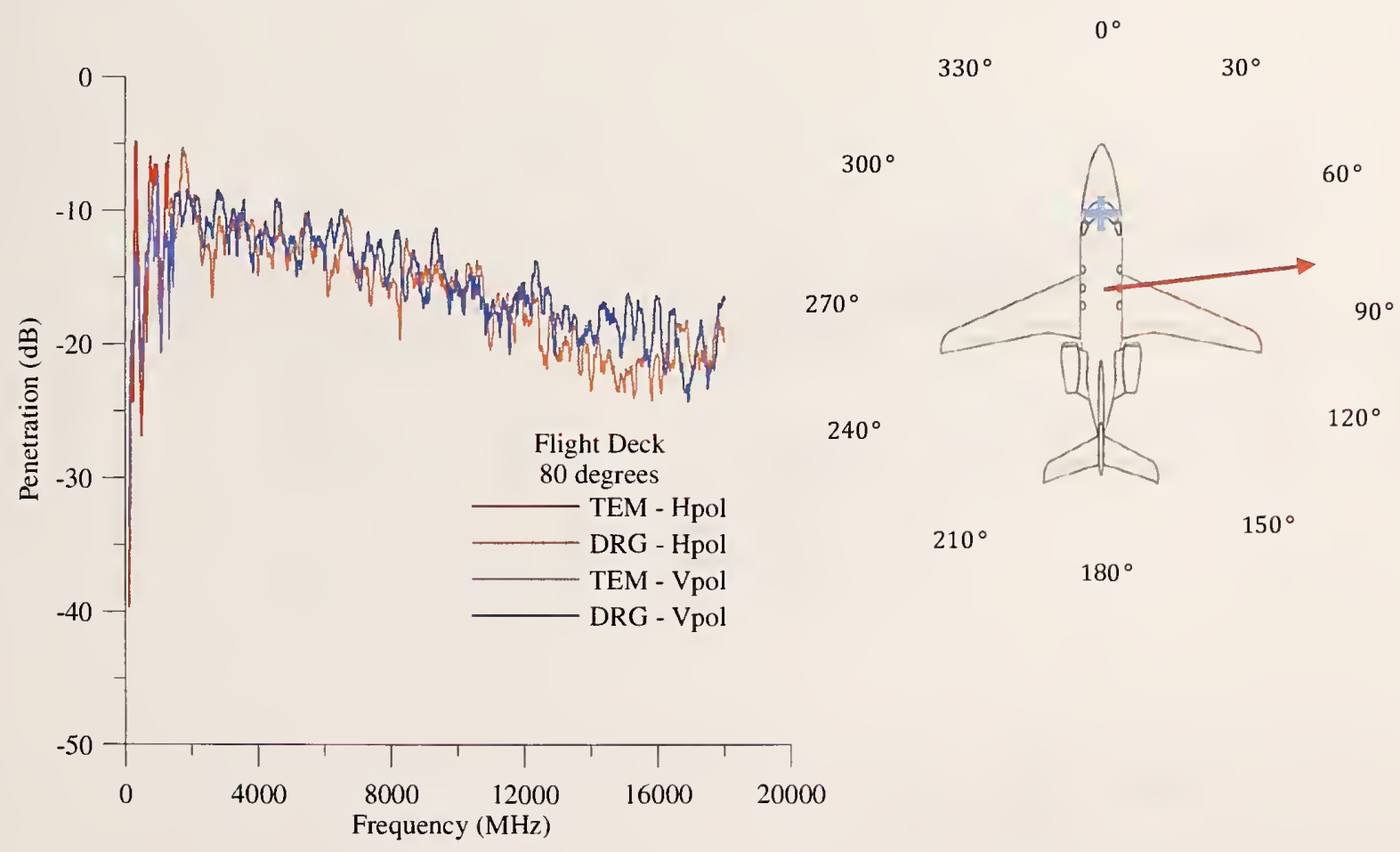

Figure 59. Penetration for all antennas and polarizations for the transmitting tower placed at the $80^{\circ}$ position and the receiving antennas placed in the flight deck. 


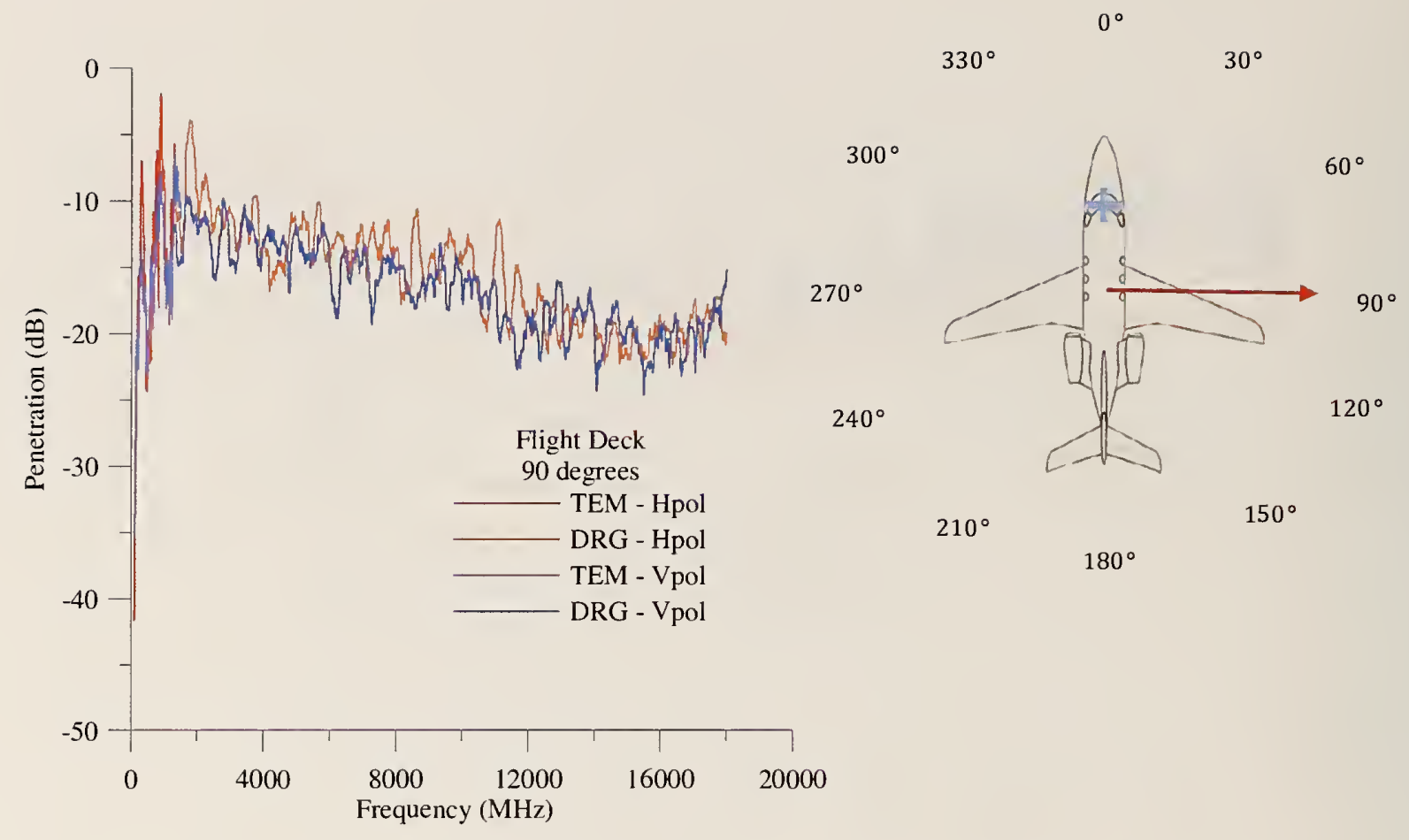

Figure 60. Penetration for all antennas and polarizations for the transmitting tower placed at the $90^{\circ}$ position and the receiving antennas placed in the flight deck.

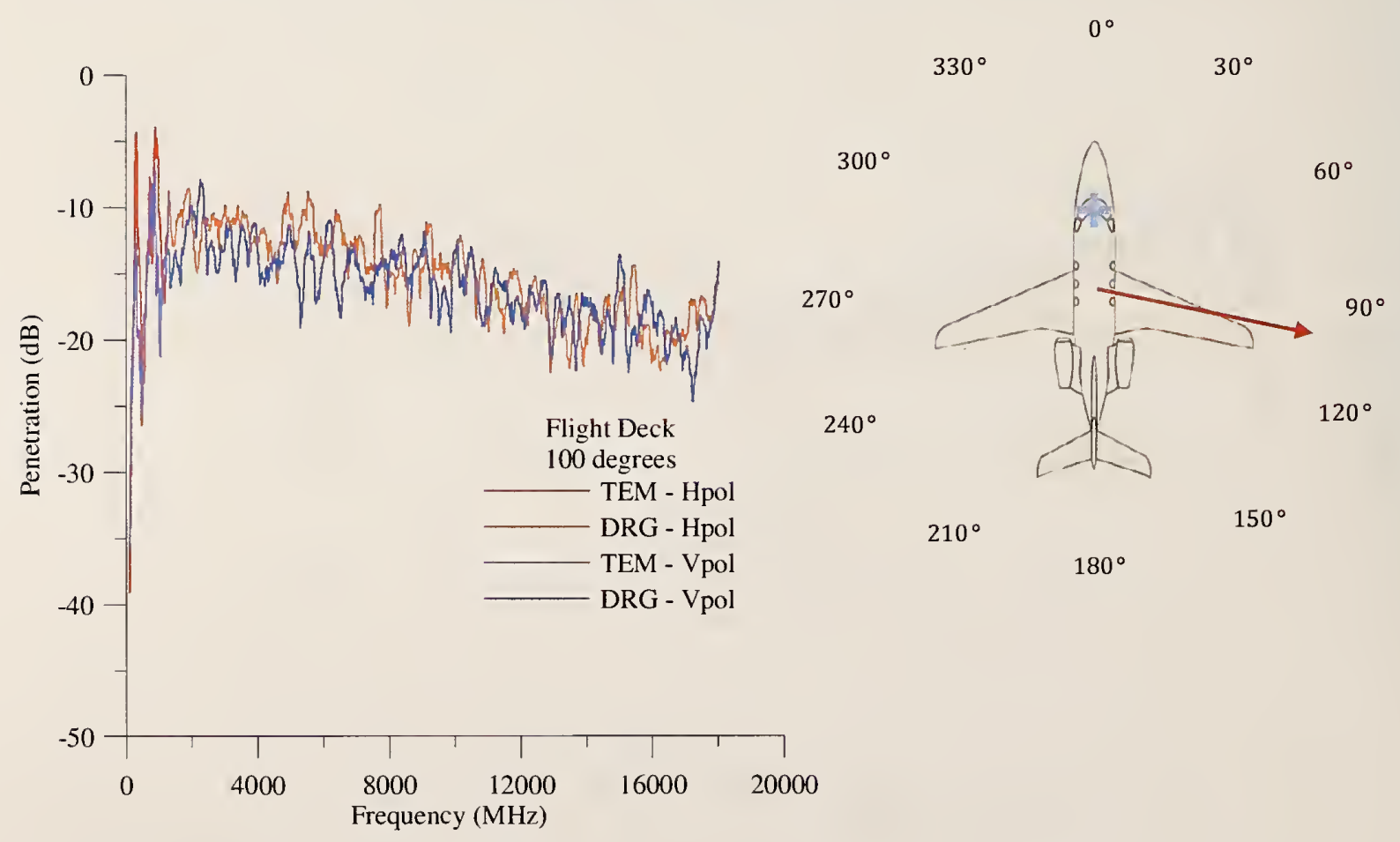

Figure 61. Penetration for all antennas and polarizations for the transmitting tower placed at the $100^{\circ}$ position and the receiving antennas placed in the flight deck. 


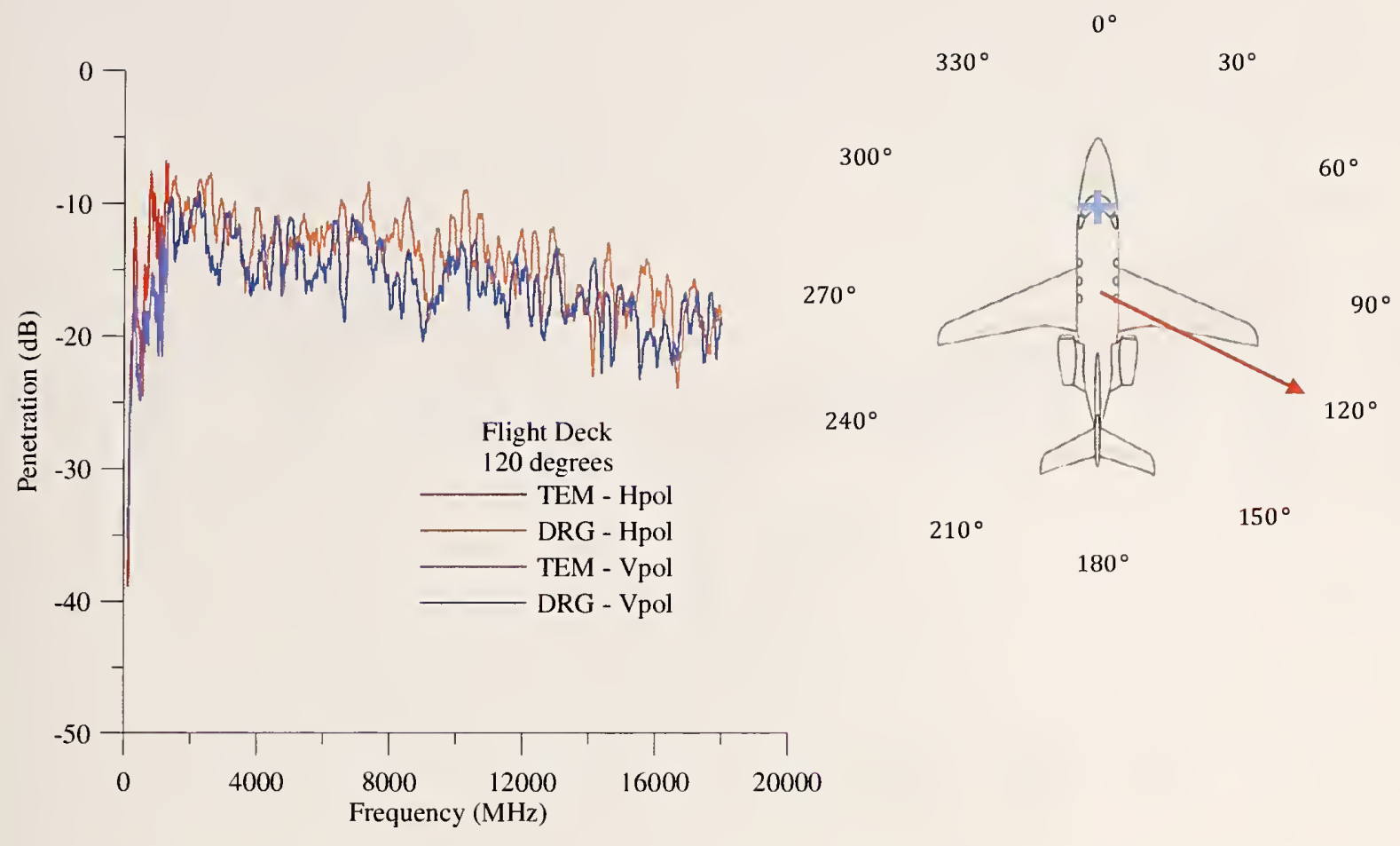

Figure 62. Penetration for all antennas and polarizations for the transmitting tower placed at the $120^{\circ}$ position and the receiving antennas placed in the flight deck.

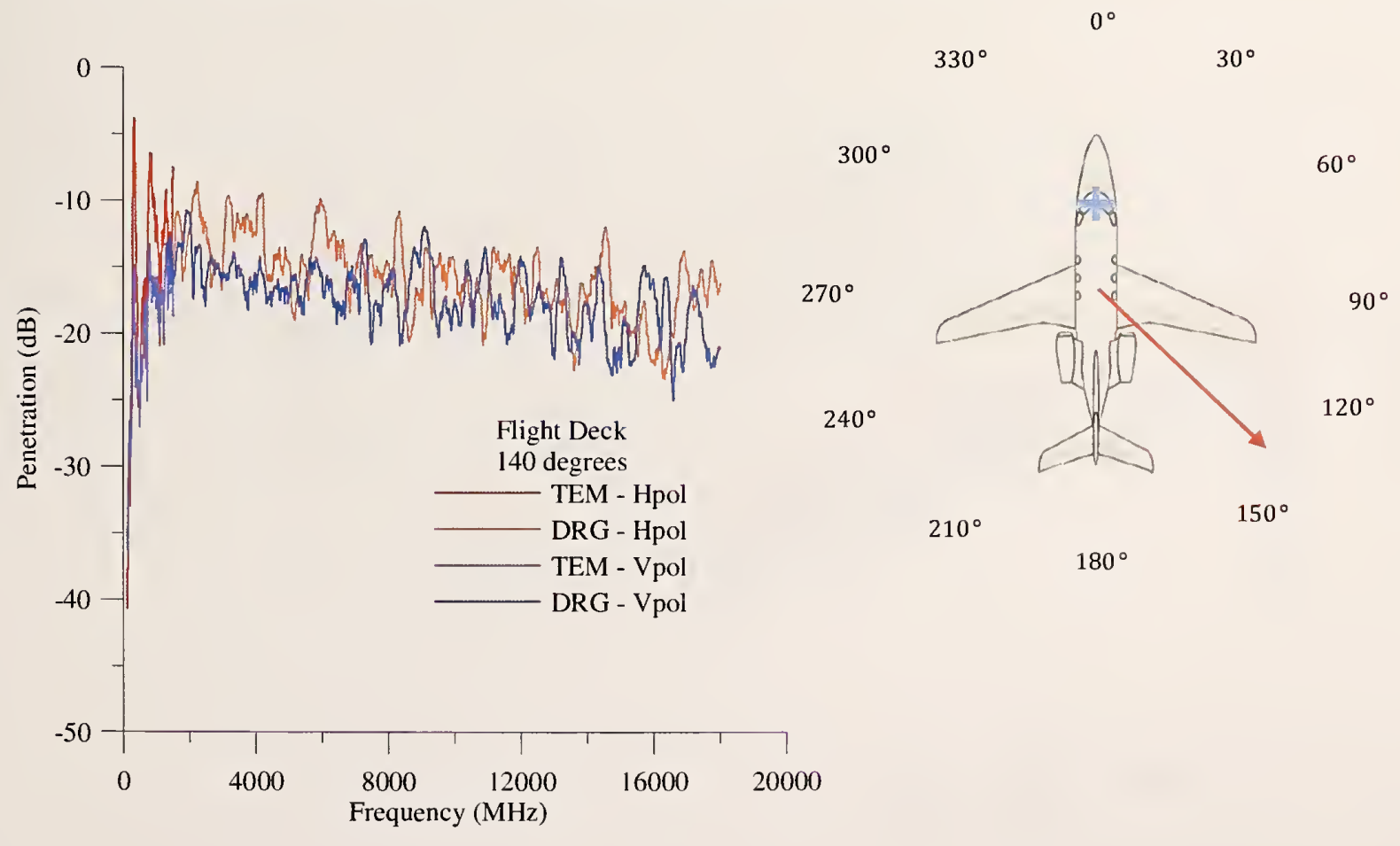

Figure 63. Penetration for all antennas and polarizations for the transmitting tower placed at the $140^{\circ}$ position and the receiving antennas placed in the flight deck. 


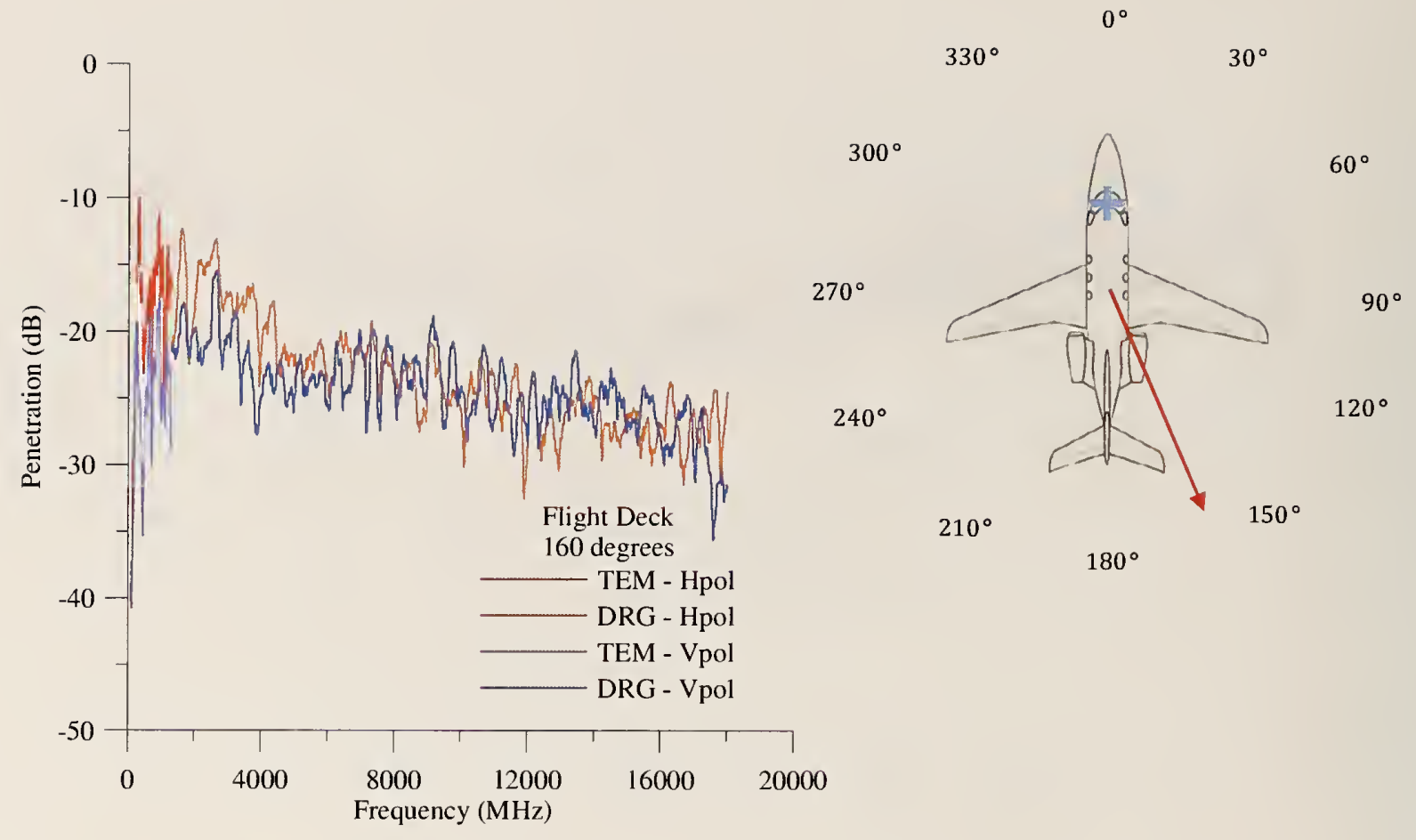

Figure 64. Penetration for all antennas and polarizations for the transmitting tower placed at the $160^{\circ}$ position and the receiving antennas placed in the flight deck.

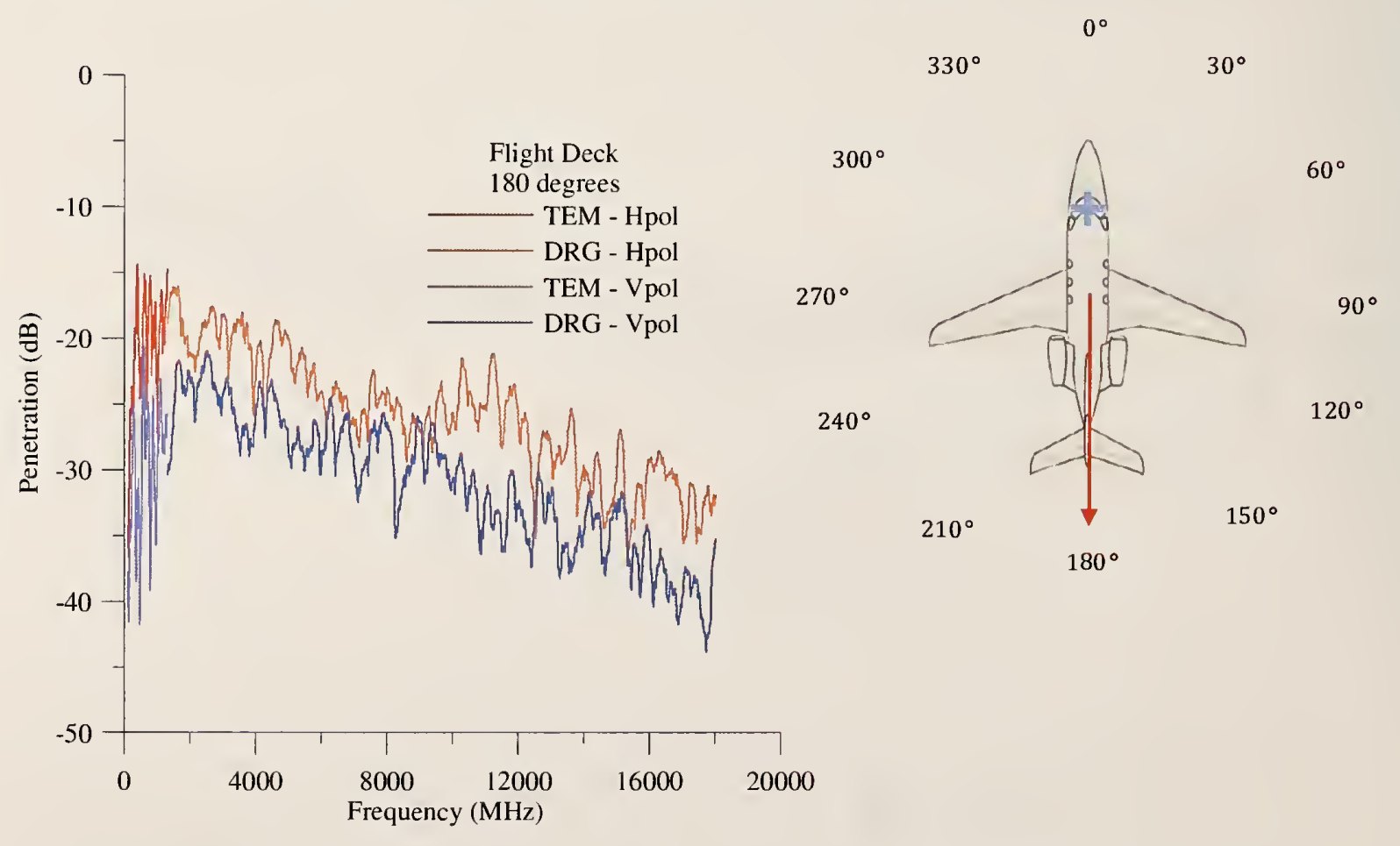

Figure 65. Penetration for all antennas and polarizations for the transmitting tower placed at the $180^{\circ}$ position and the receiving antennas placed in the flight deck. 


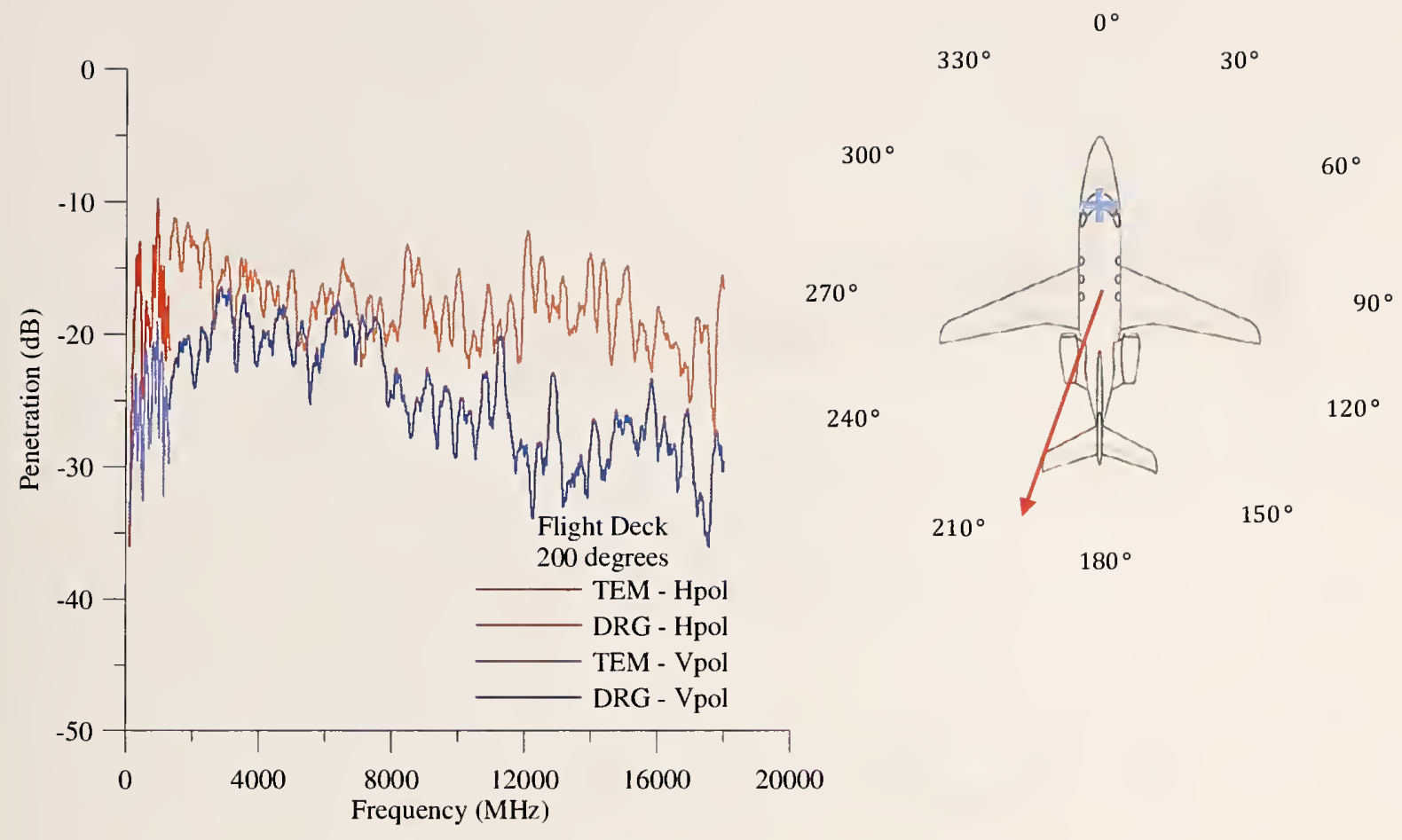

Figure 66. Penetration for all antennas and polarizations for the transmitting tower placed at the $200^{\circ}$ position and the receiving antennas placed in the flight deck.

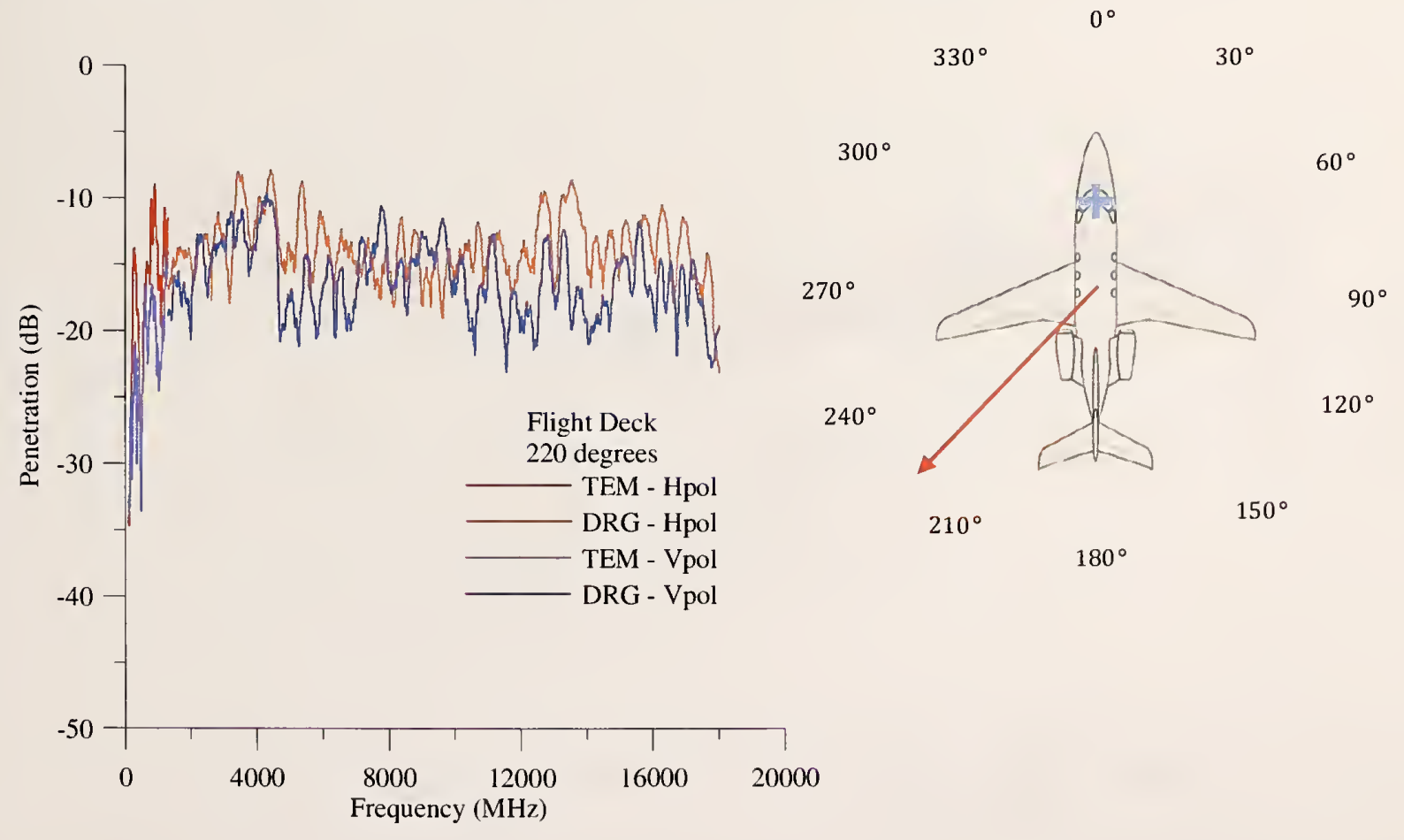

Figure 67. Penetration for all antennas and polarizations for the transmitting tower placed at the $220^{\circ}$ position and the receiving antennas placed in the flight deck. 


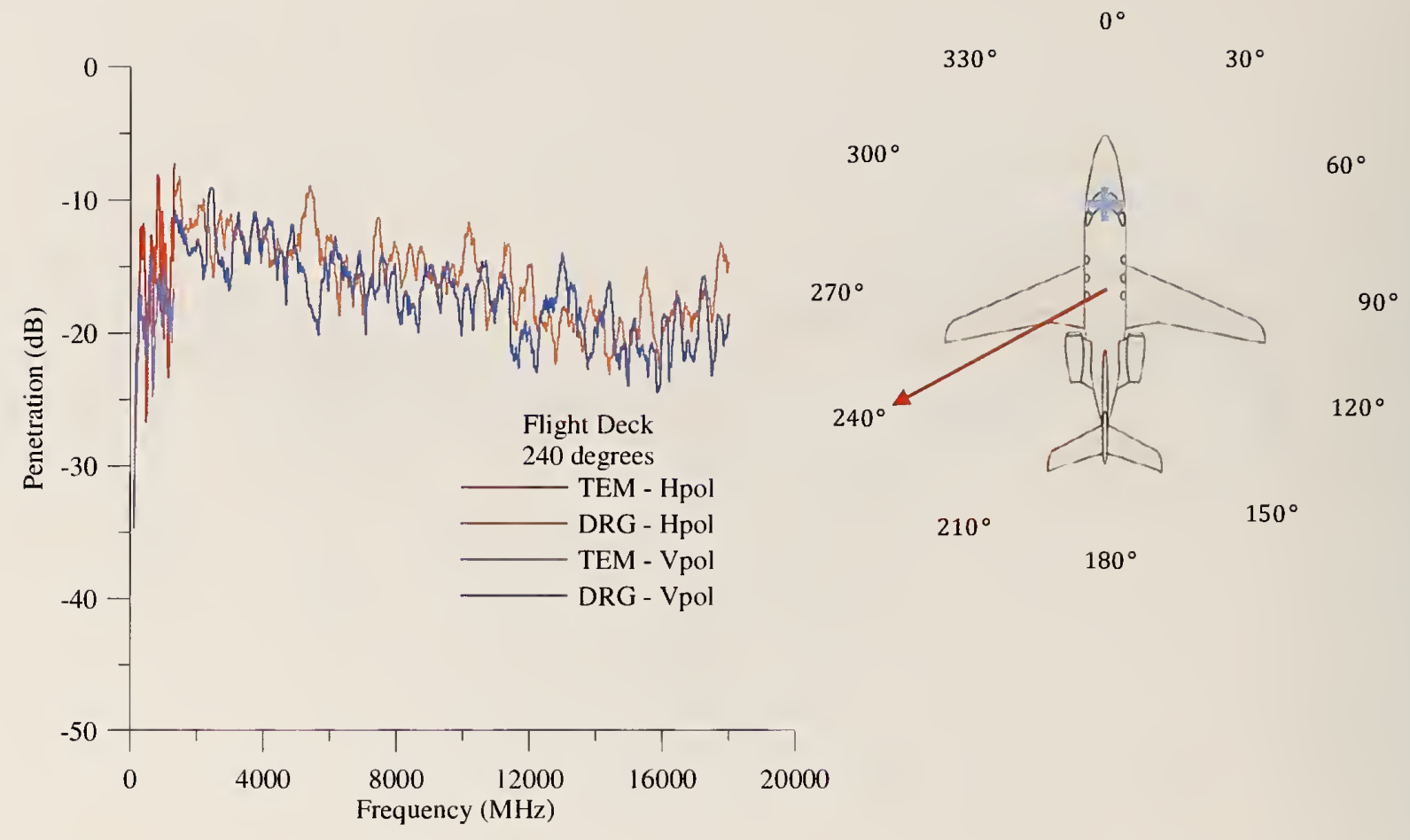

Figure 68. Penetration for all antennas and polarizations for the transmitting tower placed at the $240^{\circ}$ position and the receiving antennas placed in the flight deck.

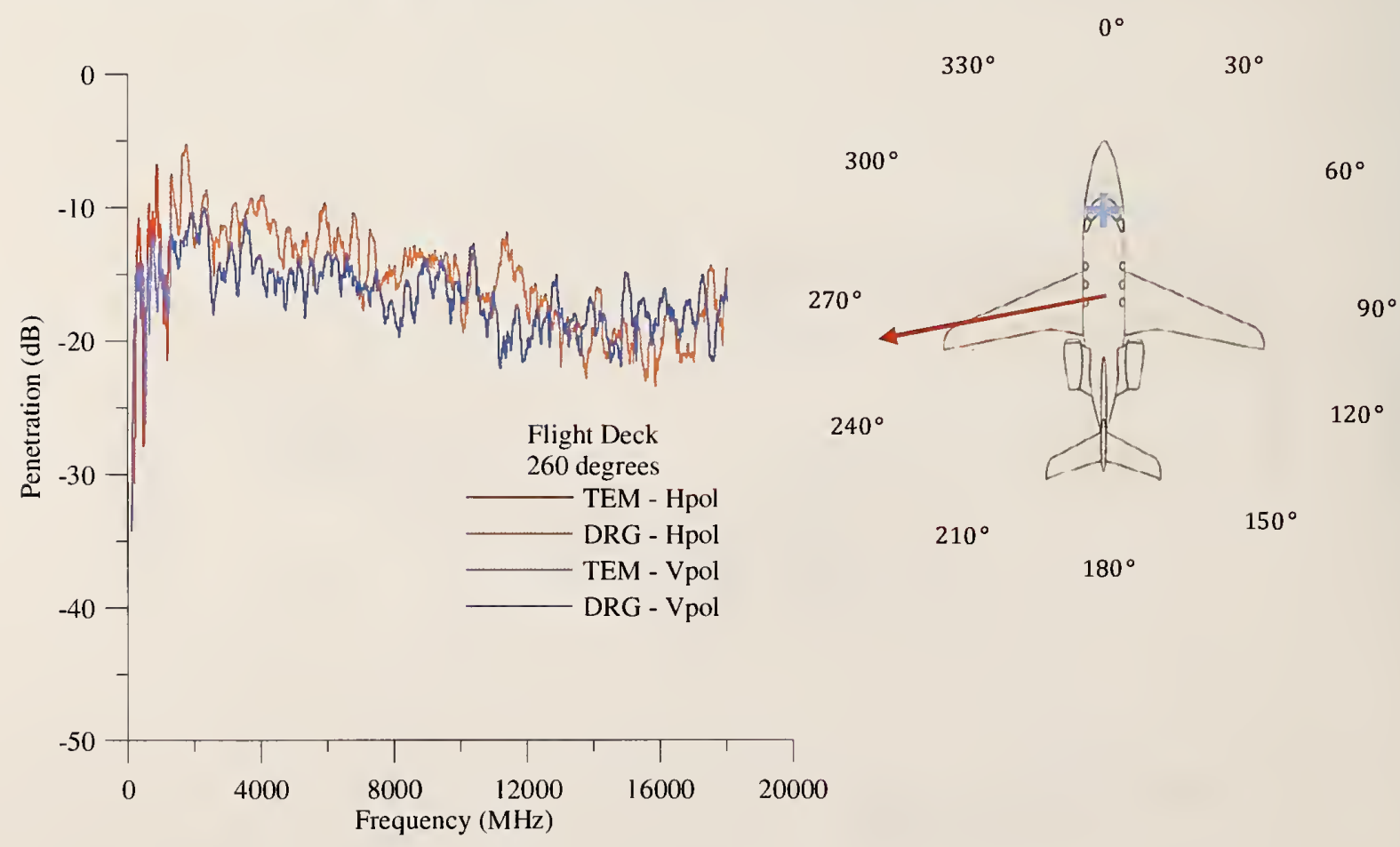

Figure 69. Penetration for all antennas and polarizations for the transmitting tower placed at the $260^{\circ}$ position and the receiving antennas placed in the flight deck. 


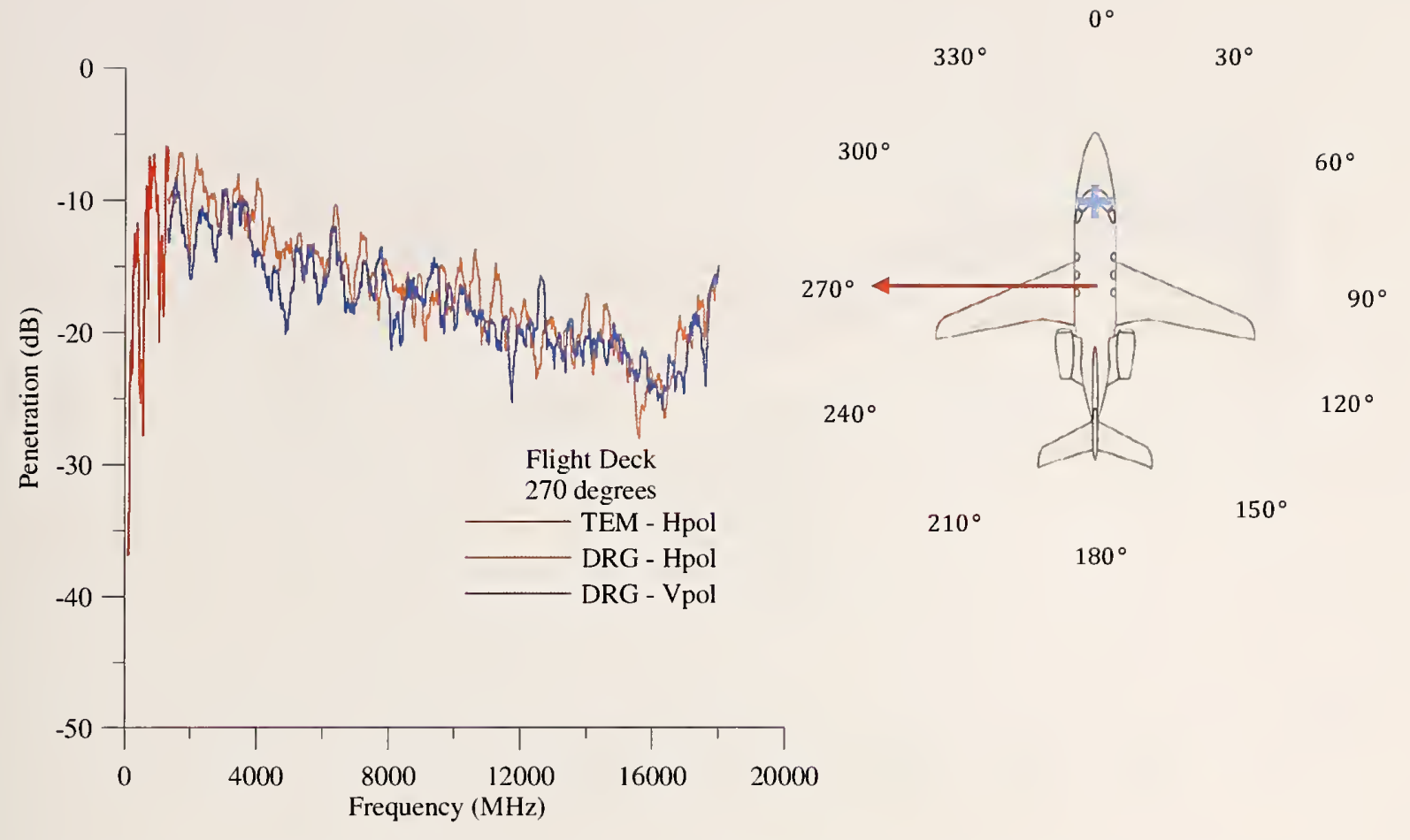

Figure 70. Penetration for all antennas and polarizations for the transmitting tower placed at the $270^{\circ}$ position and the receiving antennas placed in the flight deck.

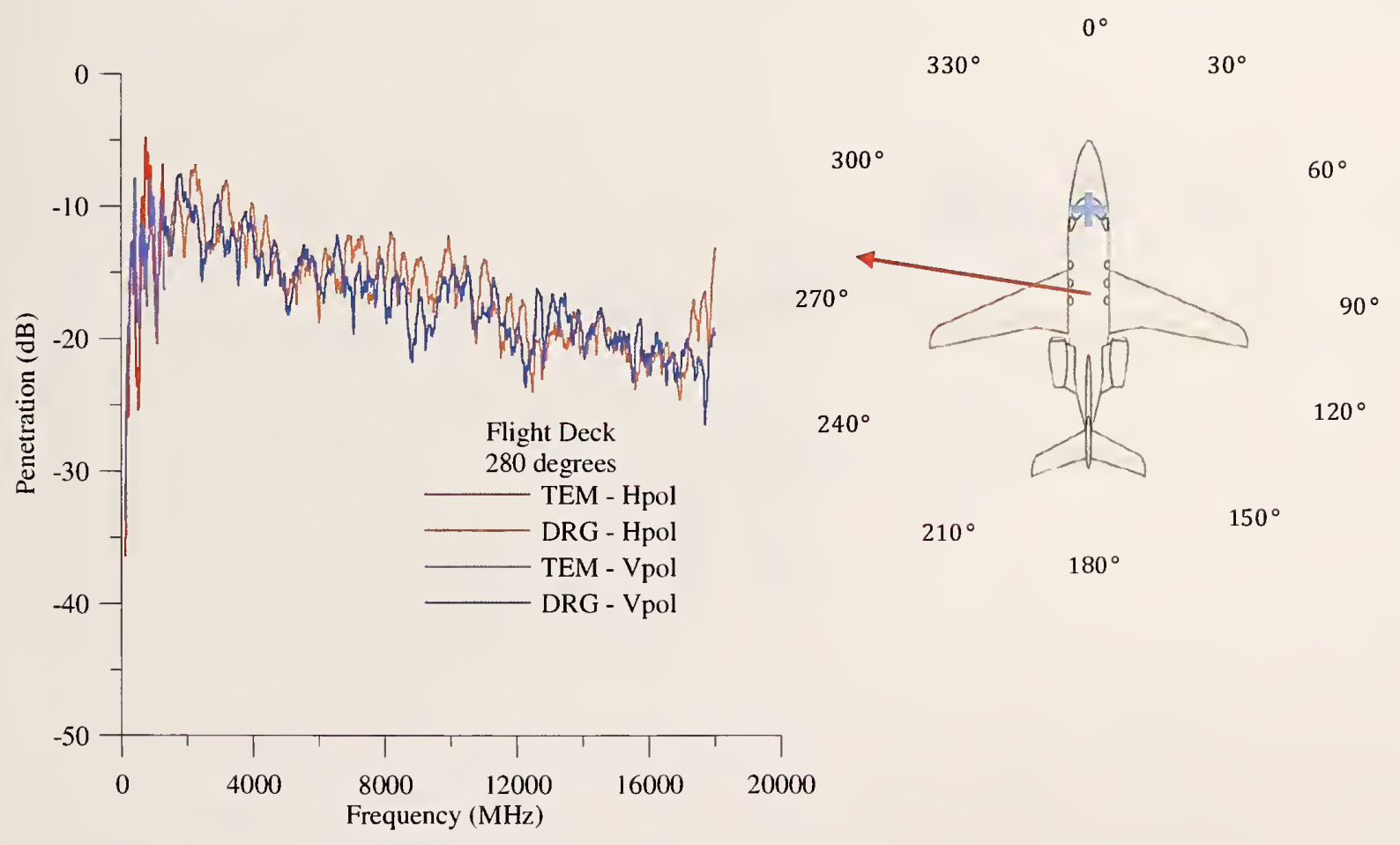

Figure 71. Penetration for all antennas and polarizations for the transmitting tower placed at the $280^{\circ}$ position and the receiving antennas placed in the flight deck. 


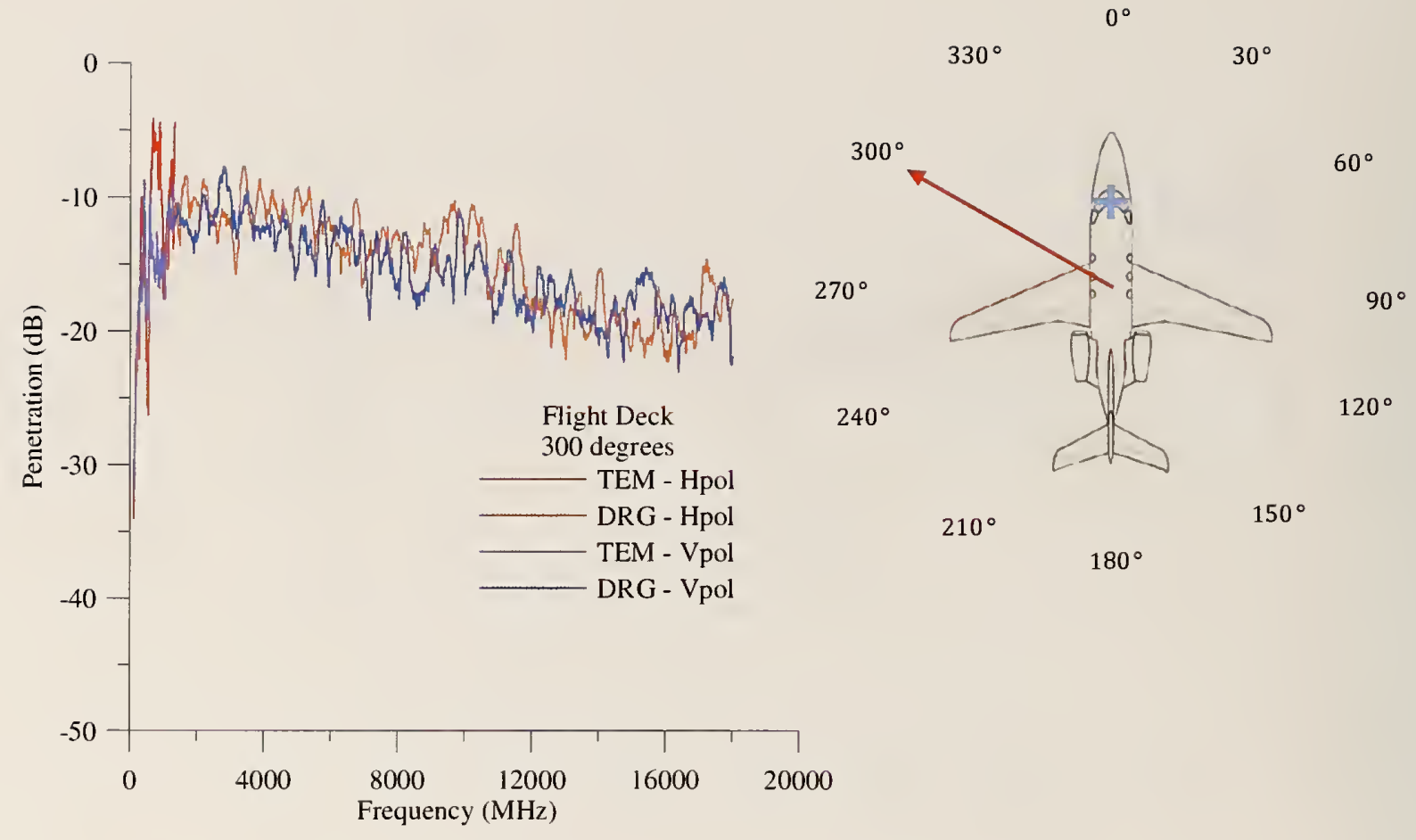

Figure 72. Penetration for all antennas and polarizations for the transmitting tower placed at the $300^{\circ}$ position and the receiving antennas placed in the flight deck.

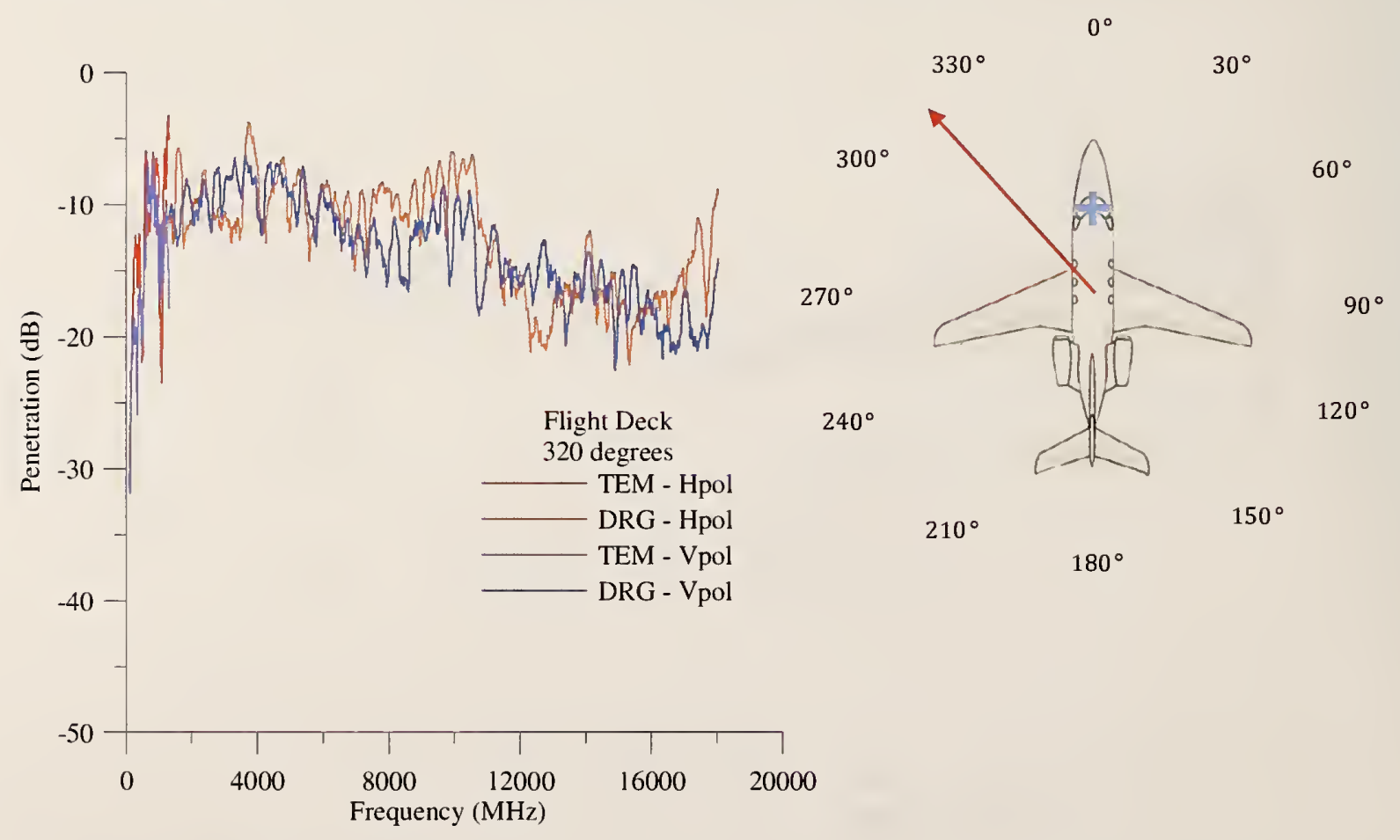

Figure 73. Penetration for all antennas and polarizations for the transmitting tower placed at the $320^{\circ}$ position and the receiving antennas placed in the flight deck. 


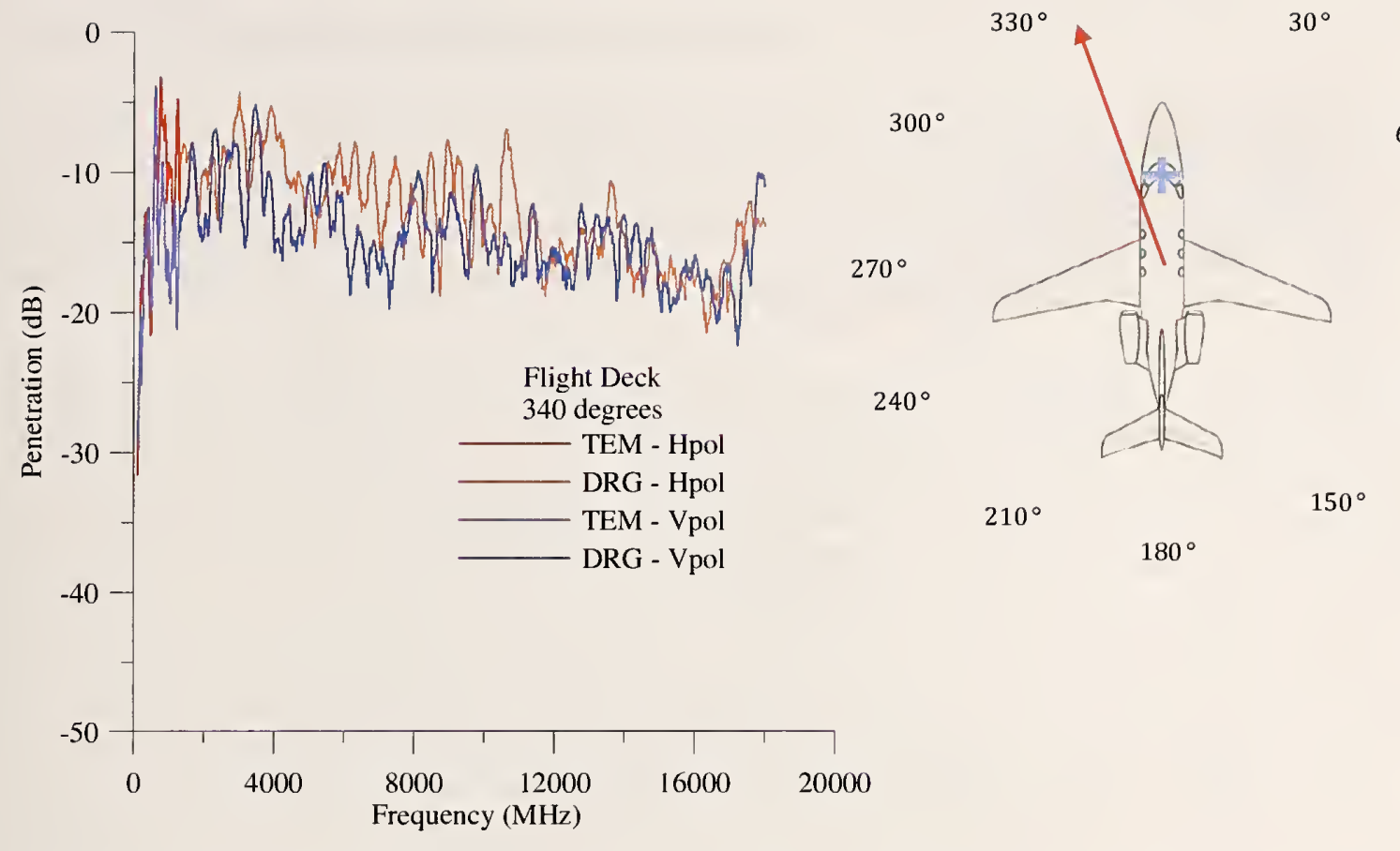

$60^{\circ}$

$90^{\circ}$

$120^{\circ}$

Figure 74. Penetration for all antennas and polarizations for the transmitting tower placed at the $340^{\circ}$ position and the receiving antennas placed in the flight deck.

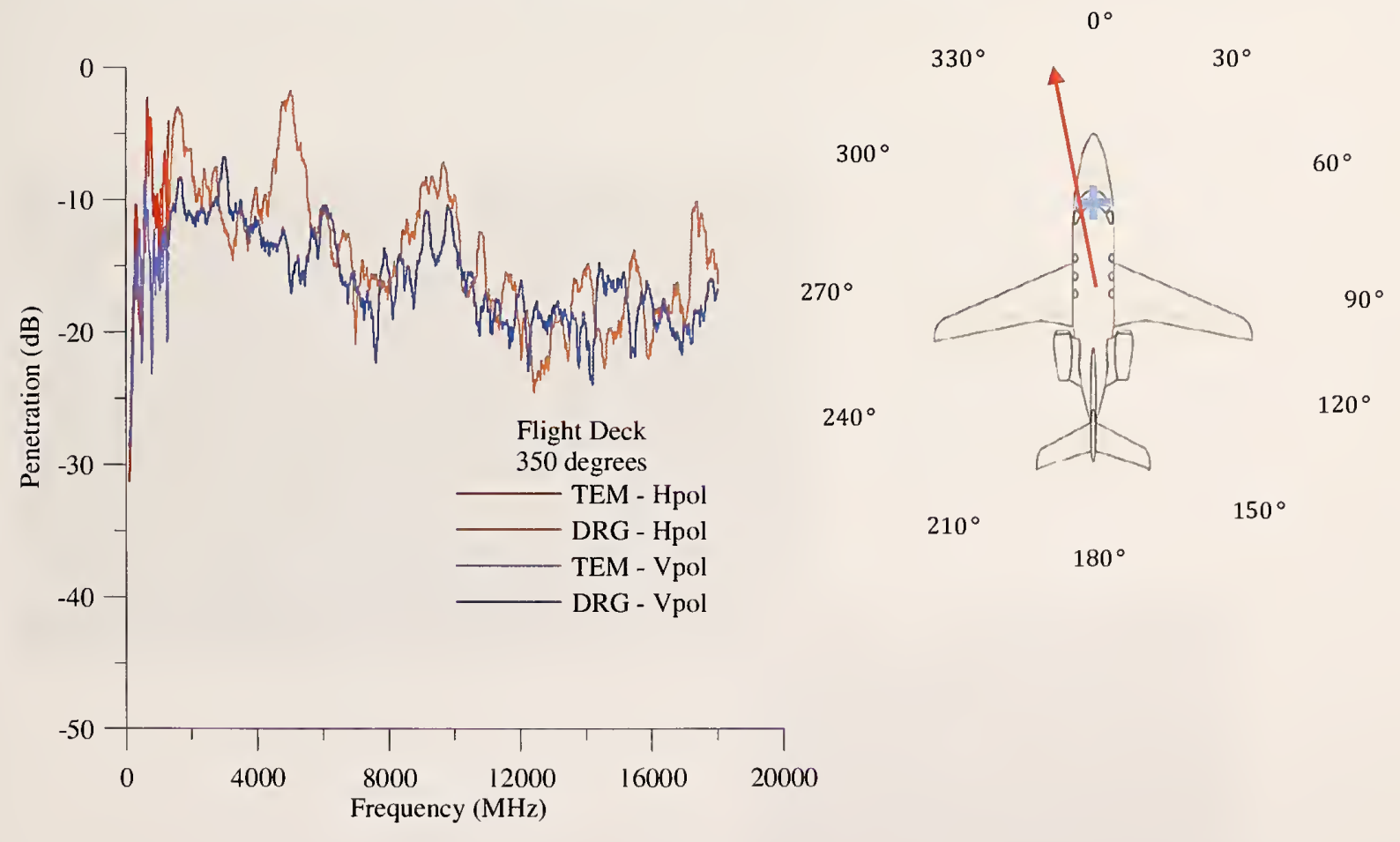

Figure 75. Penetration for all antennas and polarizations for the transmitting tower placed at the $350^{\circ}$ position and the receiving antennas placed in the flight deck. 


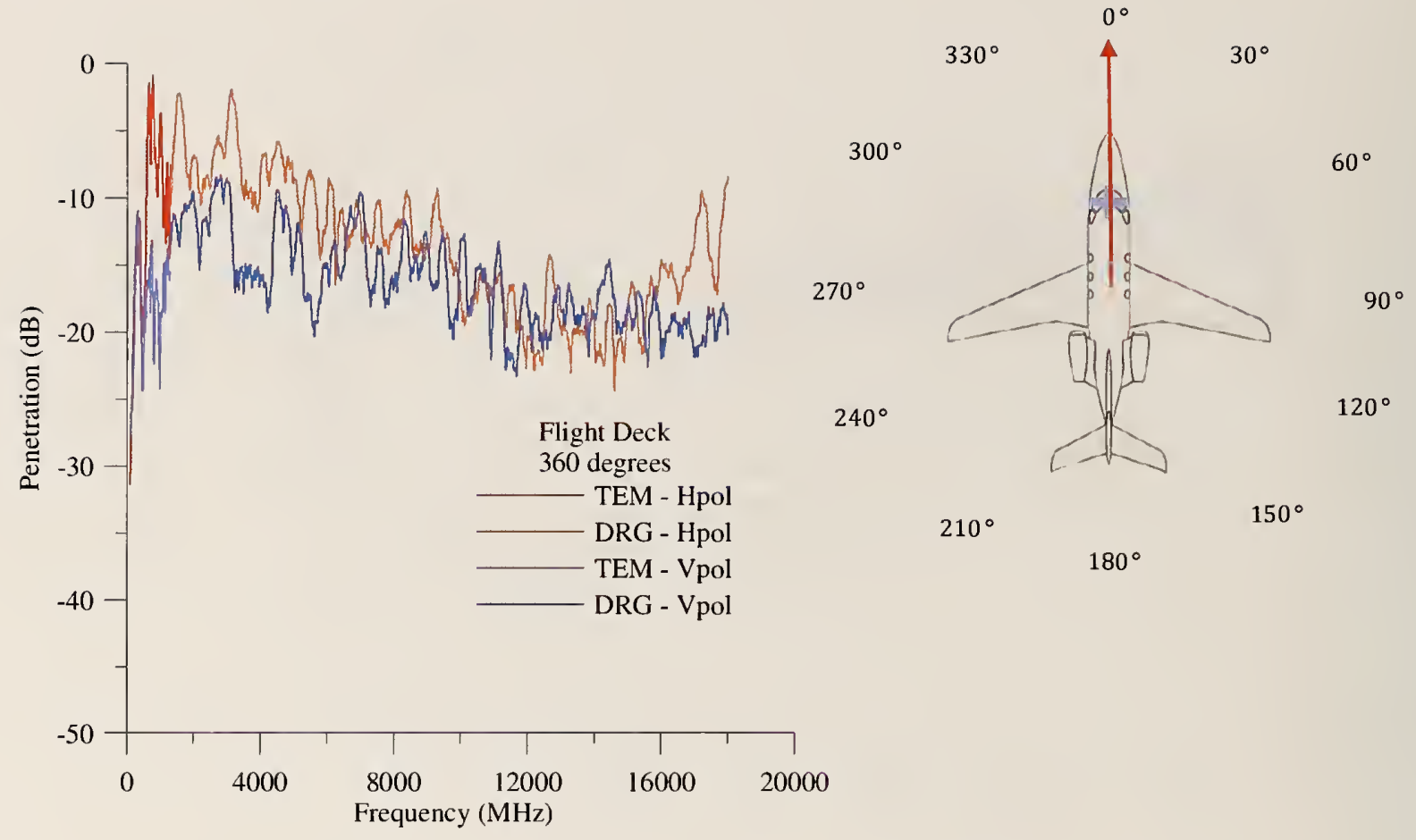

Figure 76. Penetration for all antennas and polarizations for the transmitting tower placed at the $360^{\circ}$ position and the receiving antennas placed in the flight deck. 


\subsection{Rear Cargo Hatch Penetration Results}

A series of penetration measurements was taken with the receiving antennas placed in the rear cargo hatch located at the left rear of the aircraft. The receiving antenna positions for the TEM horn antenna and DRG antenna are shown in Figure 77. The optical fiber was fed through the latch hole in the rear cargo compartment.

Figure 78 shows the time-domain plots for the DRG antenna in the horizontal polarization at various positions around the aircraft. Due to the lack of any direct coupling paths from the outside of the aircraft into the rear cargo hatch, we see no direct antenna-to-antenna coupling in these plots. However the signal levels are very asymmetric around the aircraft. Large signal levels are seen from positions $180^{\circ}$ to $270^{\circ}$, with minimum signal levels seen at the $40^{\circ}, 60^{\circ}$, and $90^{\circ}$ positions. There appears to be a large shielding object on the inner part of the rear cargo hatch. Figure 79 shows the penetration plots around the aircraft. High penetration is seen consistently around the aircraft at a frequency around $860 \mathrm{MHz}$. The lowest penetration is seen at $40^{\circ}$ and $60^{\circ}$, with average values of $-30 \mathrm{~dB}$ (horizontal polarization) and $-35 \mathrm{~dB}$ (vertical polarization) for frequencies below $4000 \mathrm{MHz}$, and average values of $-35 \mathrm{~dB}$ (horizontal polarization) and $-40 \mathrm{~dB}$ (vertical polarization) for frequencies above this. Highest penetration values are seen at an angle of $270^{\circ}$ for both horizontal and vertical polarizations of approximately $-15 \mathrm{~dB}$ to $-17 \mathrm{~dB}$ across the frequency band. We have not seen this type of pattern in other aircraft. There is a distinct broadband resonance at around $8000 \mathrm{MHz}$ for the $100^{\circ}$ to $140^{\circ}$ positions. Penetration results at every external angle are shown in Figures 80 to 99 .

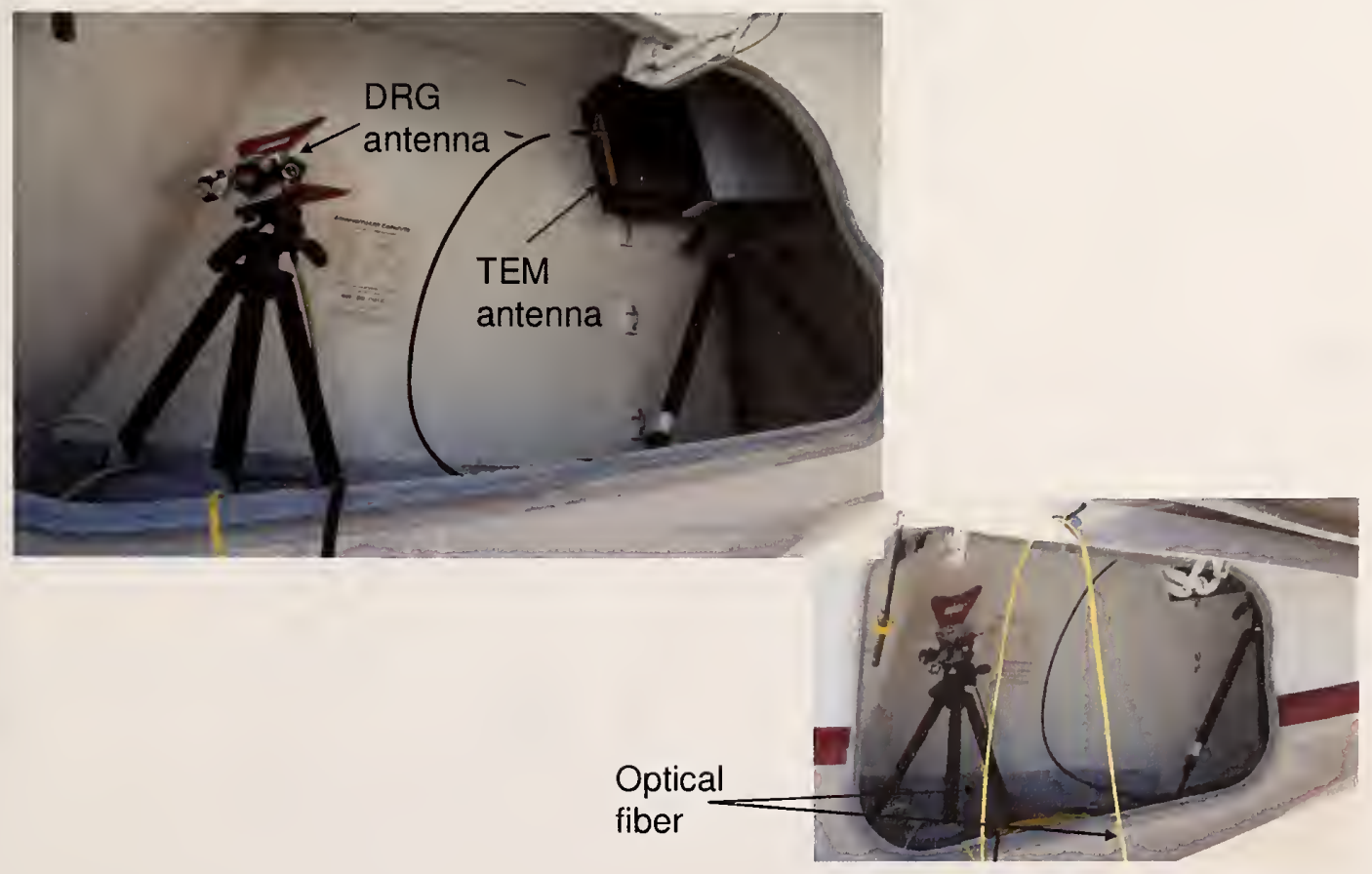

Figure 77. DRG and TEM antenna placement in the rear cargo hatch. 


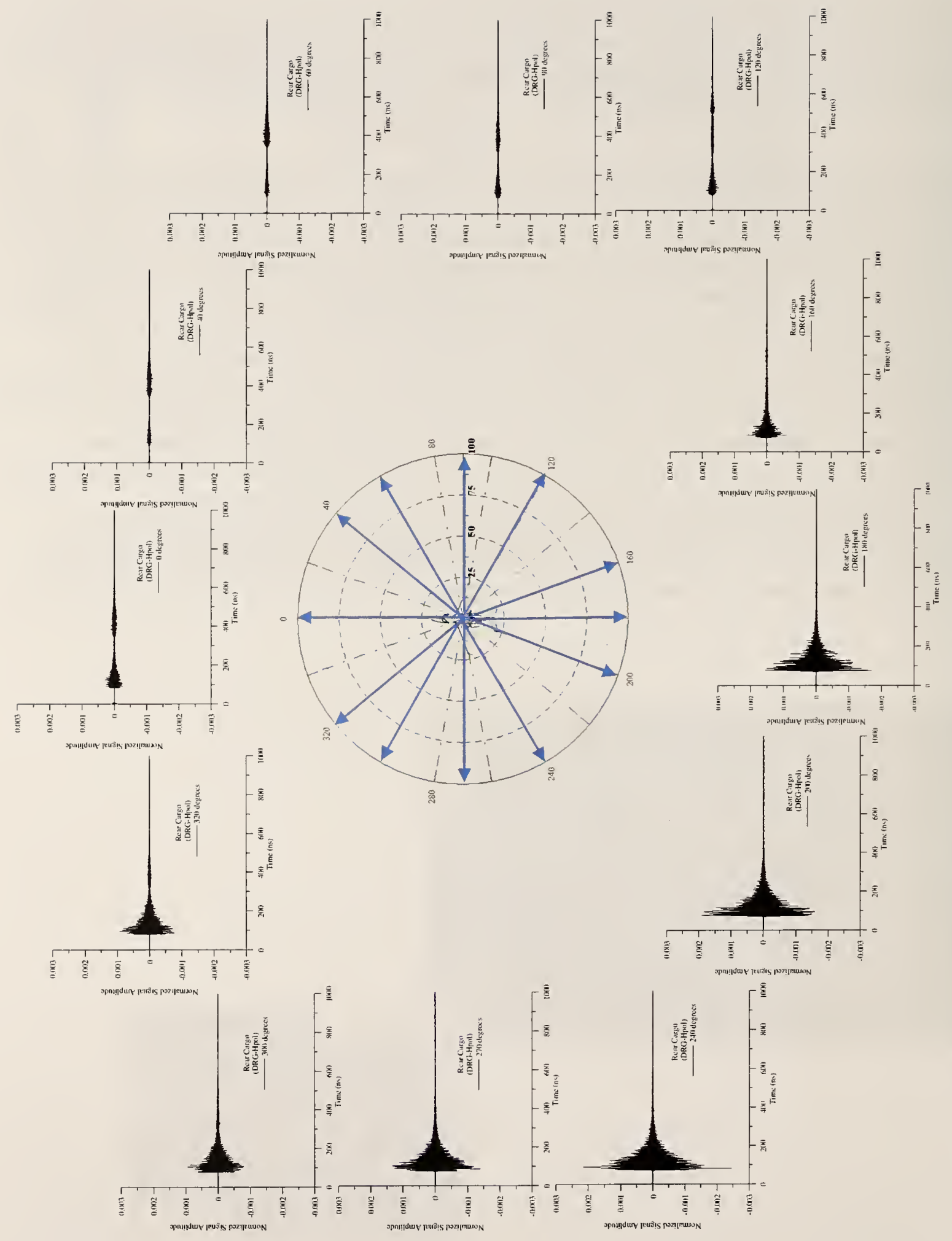

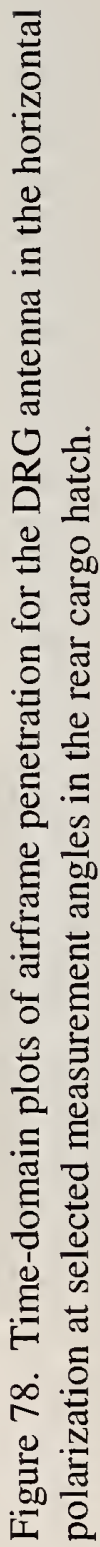




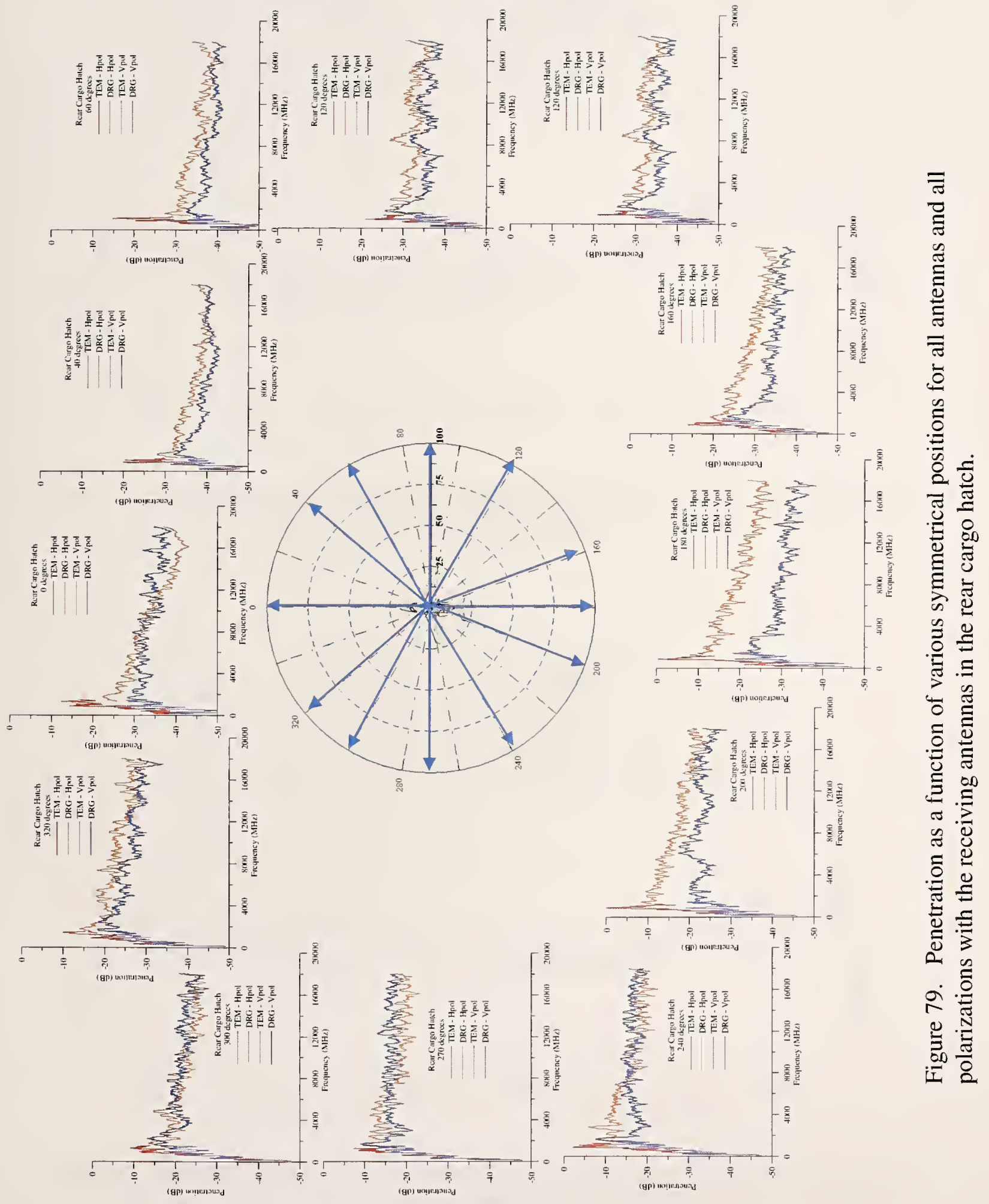




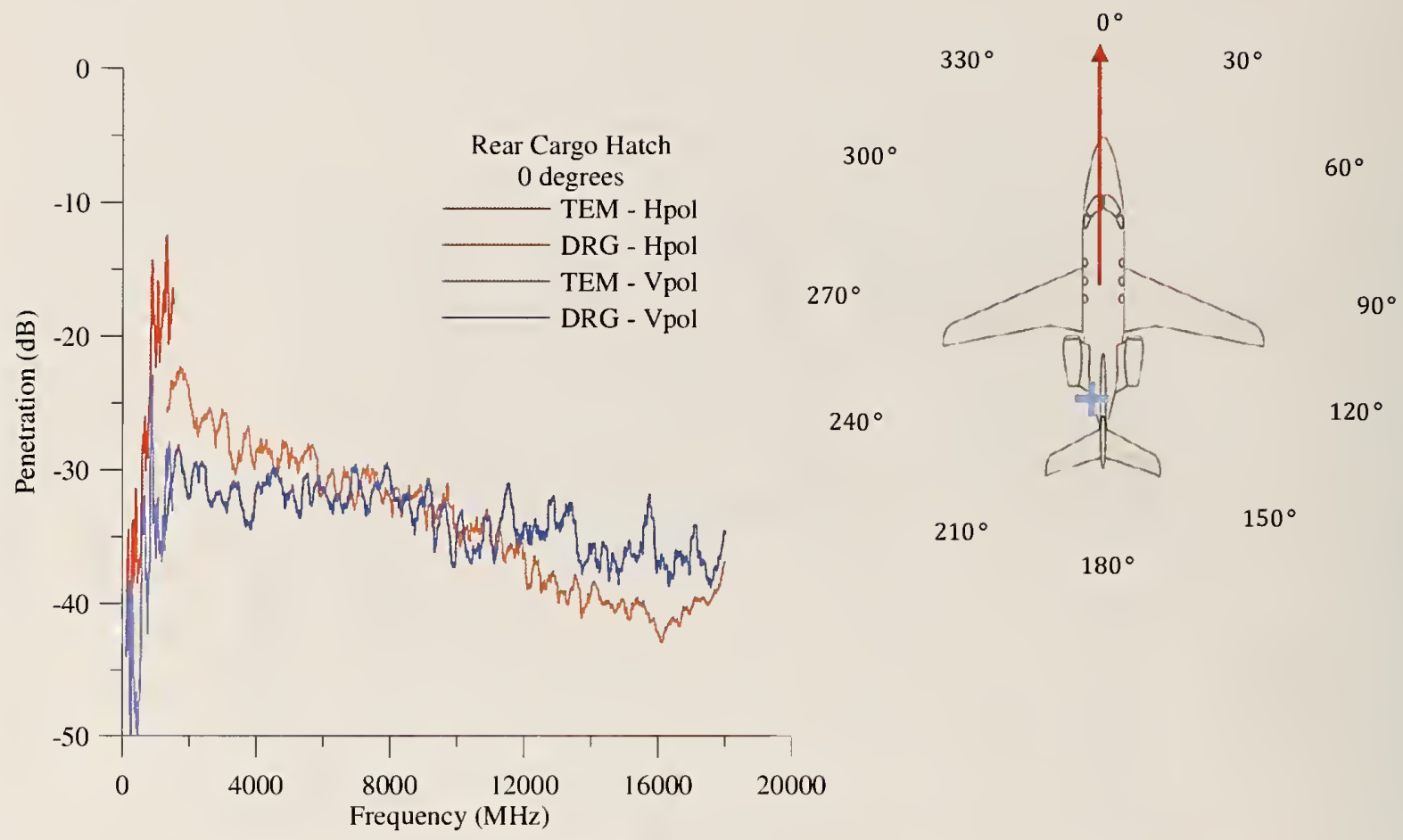

Figure 80. Penetration for all antennas and polarizations for the transmitting tower placed at the $0^{\circ}$ position and the receiving antennas placed in the rear cargo hatch.

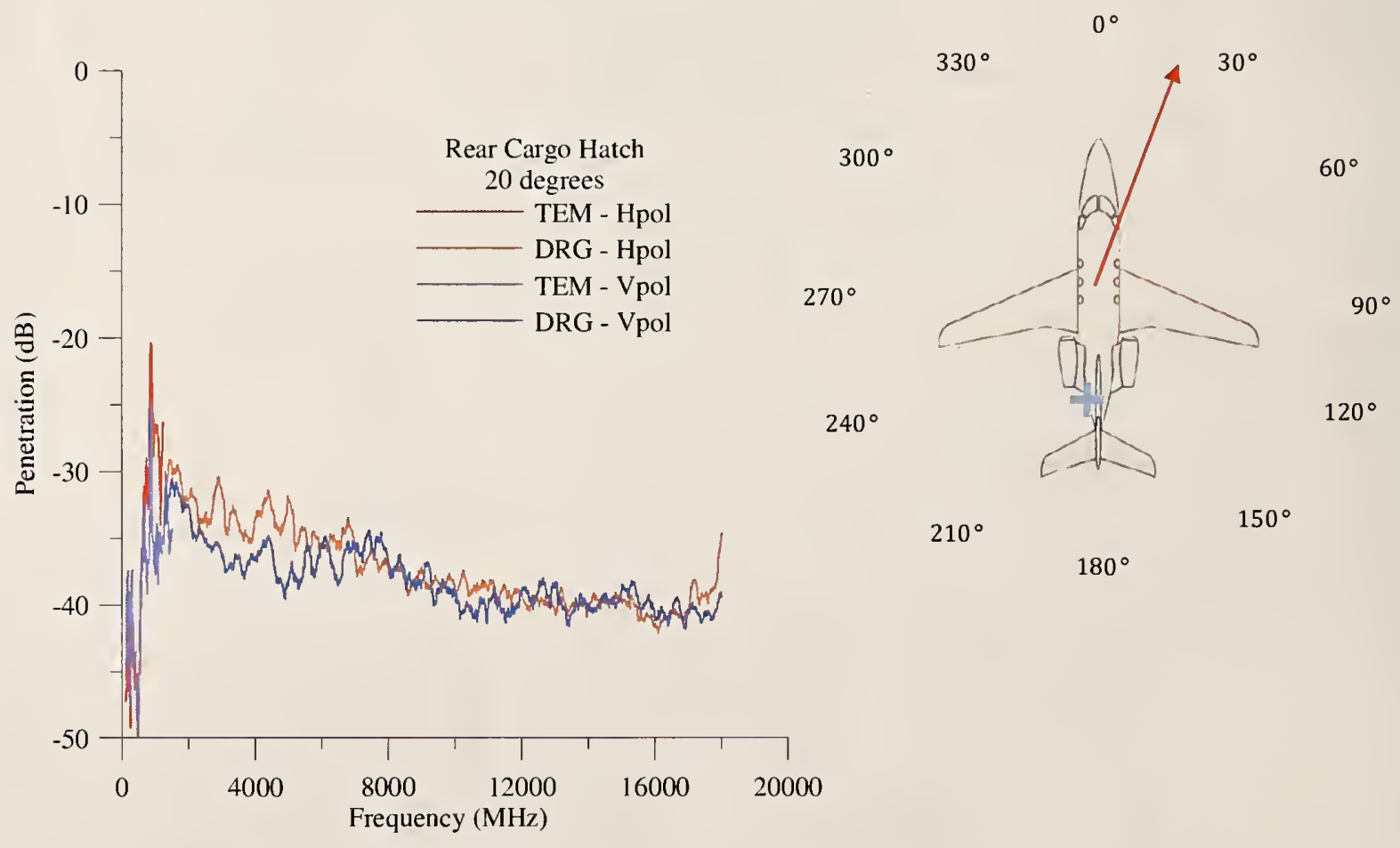

Figure 81. Penetration for all antennas and polarizations for the transmitting tower placed at the $20^{\circ}$ position and the receiving antennas placed in the rear cargo hatch. 


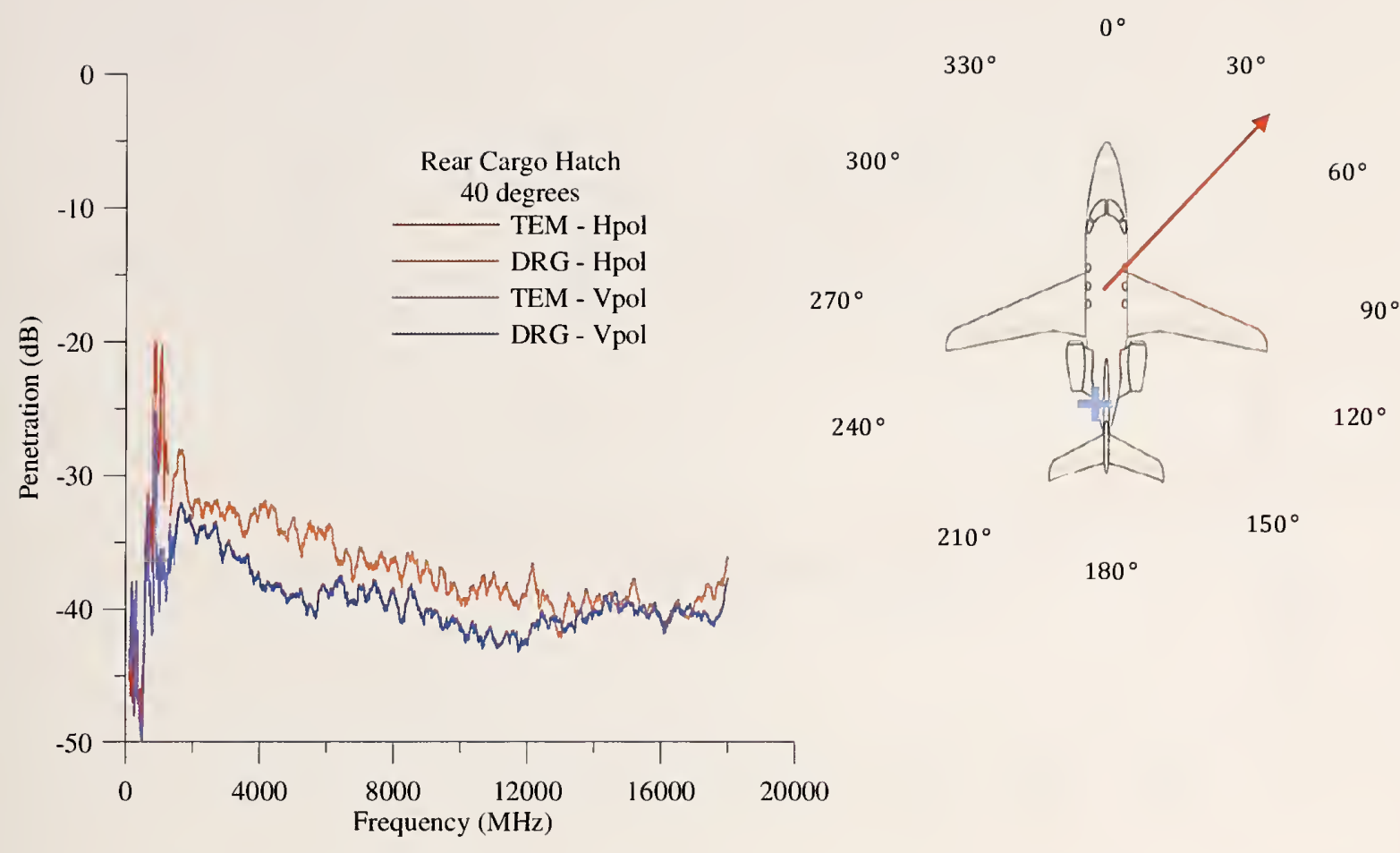

Figure 82. Penetration for all antennas and polarizations for the transmitting tower placed at the $40^{\circ}$ position and the receiving antennas placed in the rear cargo hatch.

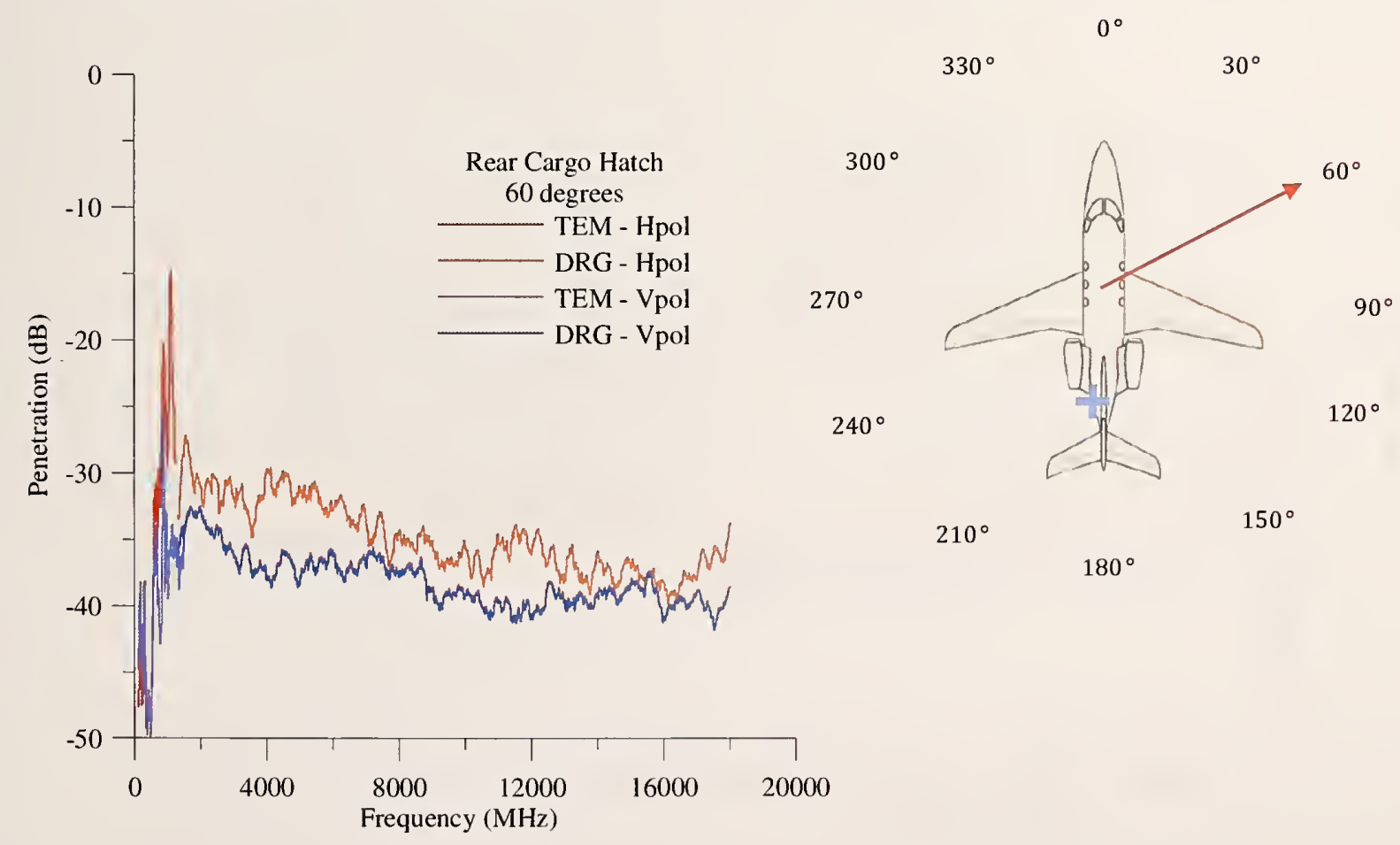

Figure 83. Penetration for all antennas and polarizations for the transmitting tower placed at the $60^{\circ}$ position and the receiving antennas placed in the rear cargo hatch. 


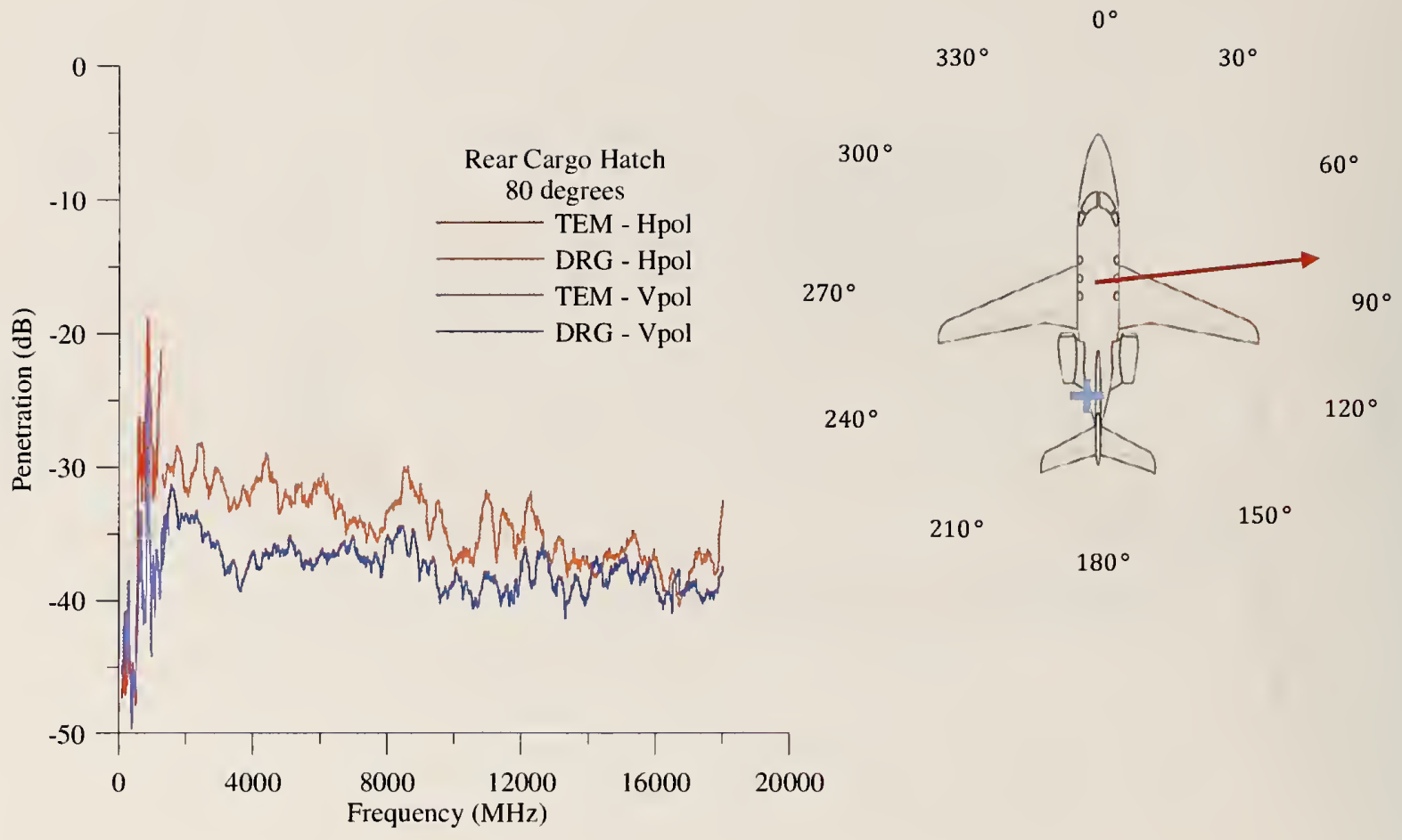

Figure 84. Penetration for all antennas and polarizations for the transmitting tower placed at the $80^{\circ}$ position and the receiving antennas placed in the rear cargo hatch.

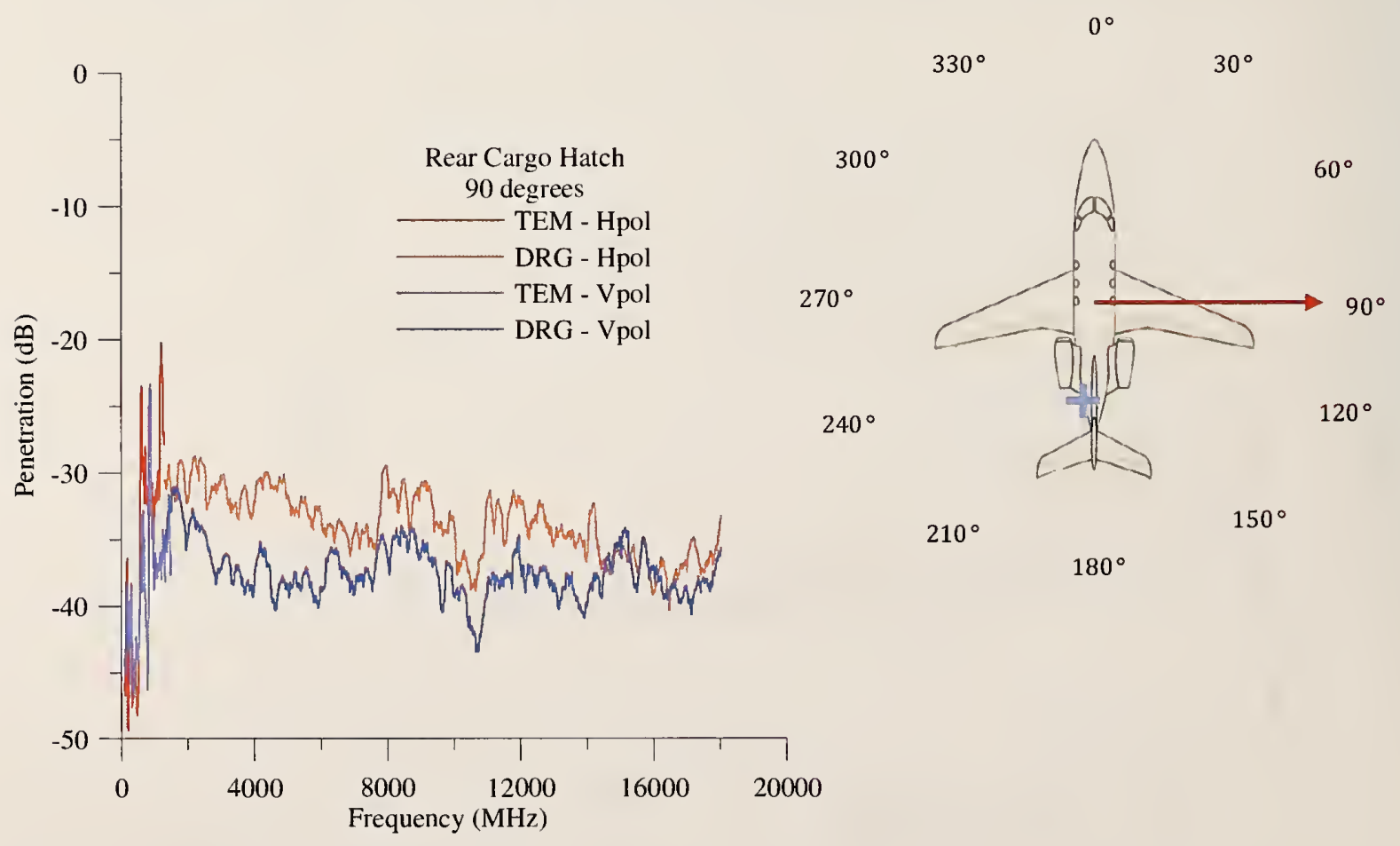

Figure 85. Penetration for all antennas and polarizations for the transmitting tower placed at the $90^{\circ}$ position and the receiving antennas placed in the rear cargo hatch. 


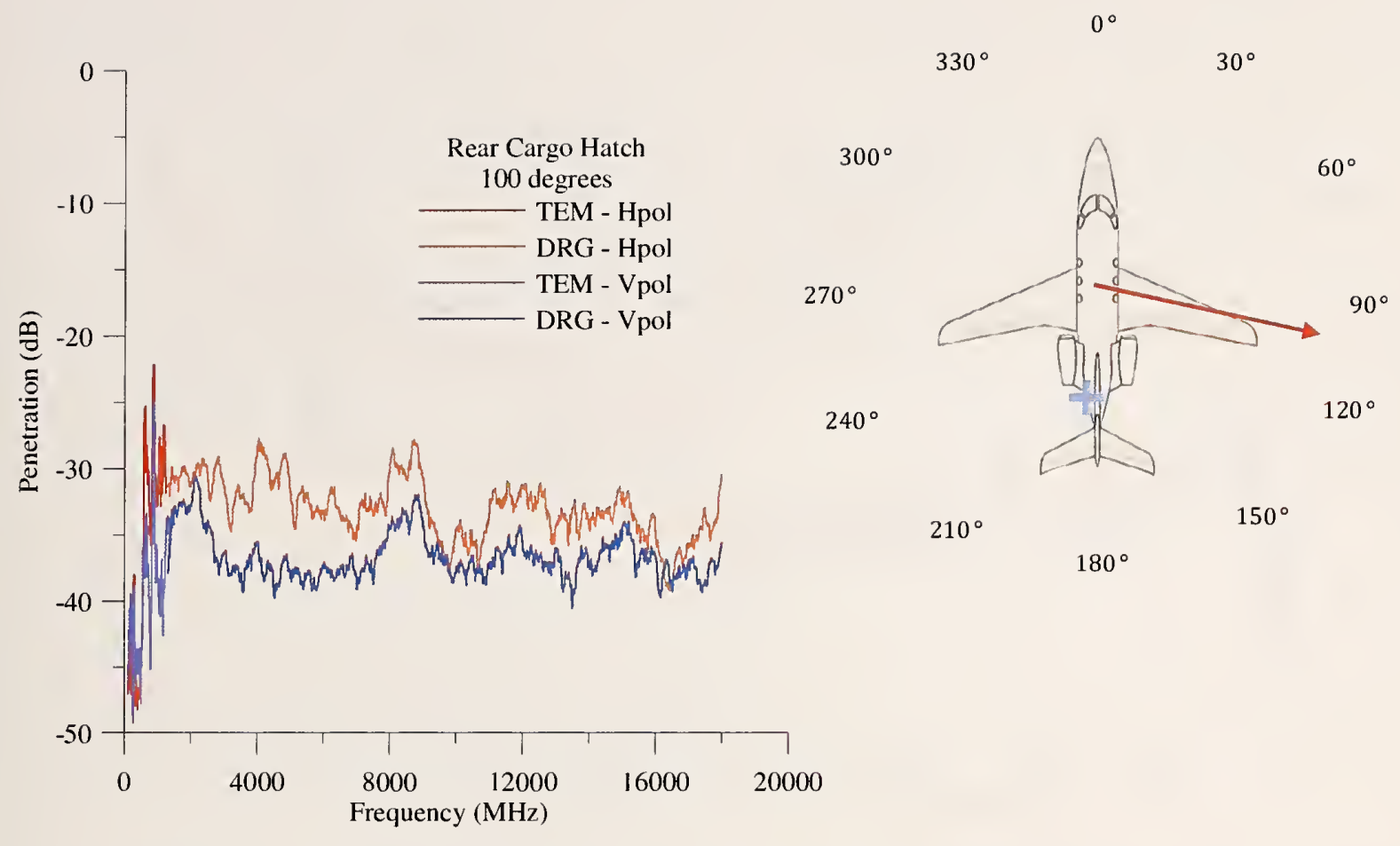

Figure 86. Penetration for all antennas and polarizations for the transmitting tower placed at the $100^{\circ}$ position and the receiving antennas placed in the rear cargo hatch.

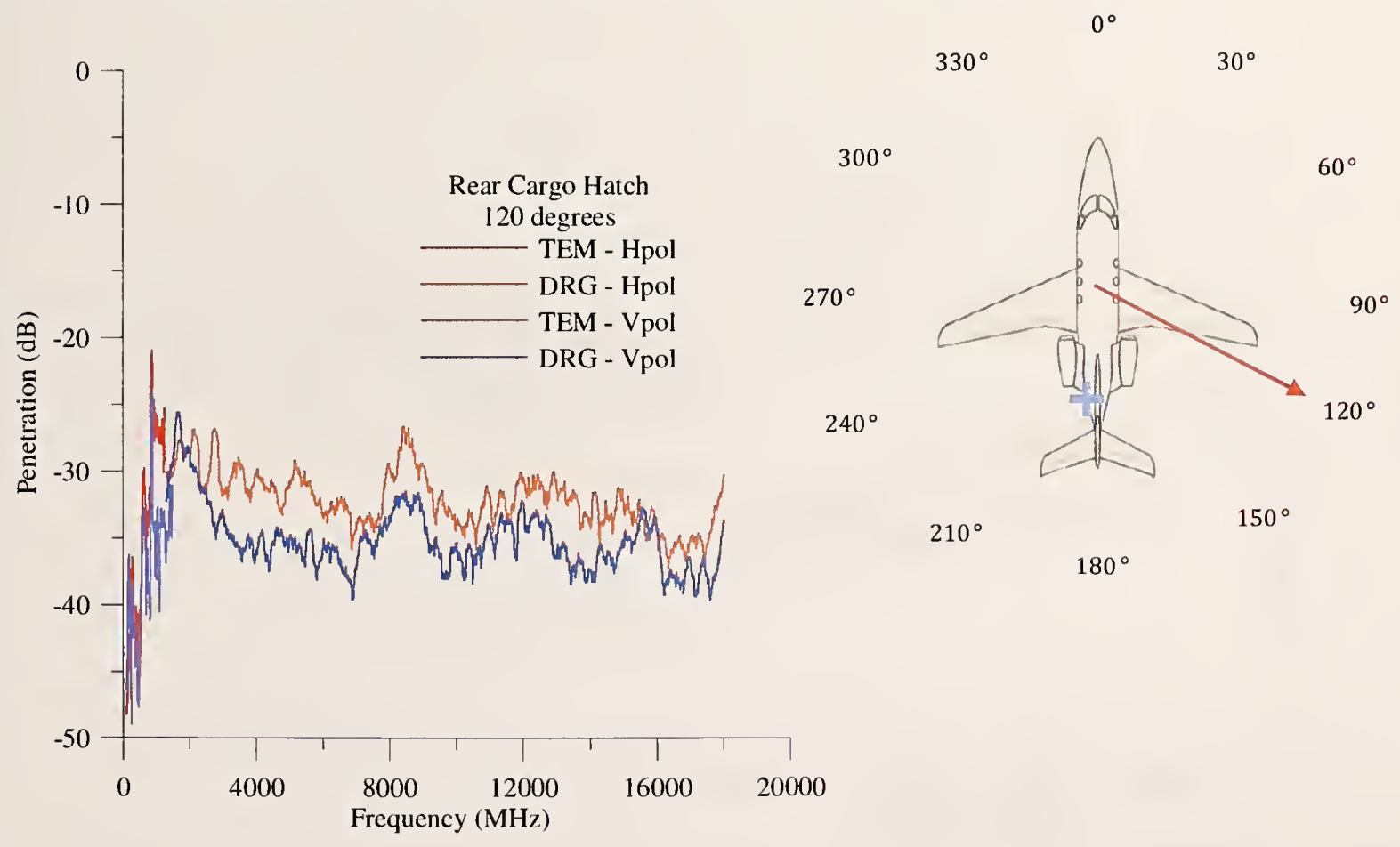

Figure 87. Penetration for all antennas and polarizations for the transmitting tower placed at the $120^{\circ}$ position and the receiving antennas placed in the rear cargo hatch. 


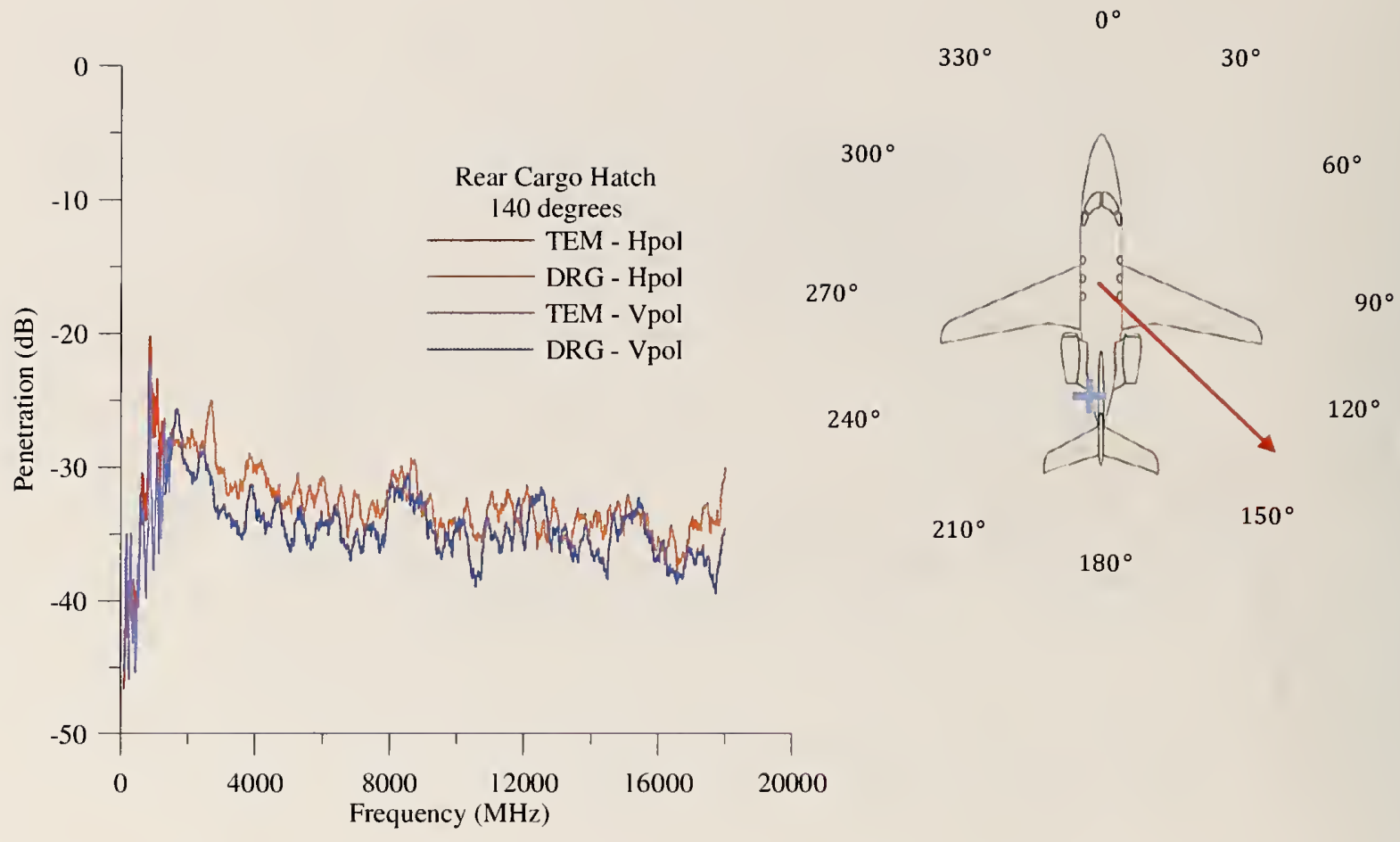

Figure 88. Penetration for all antennas and polarizations for the transmitting tower placed at the $140^{\circ}$ position and the receiving antennas placed in the rear cargo hatch.

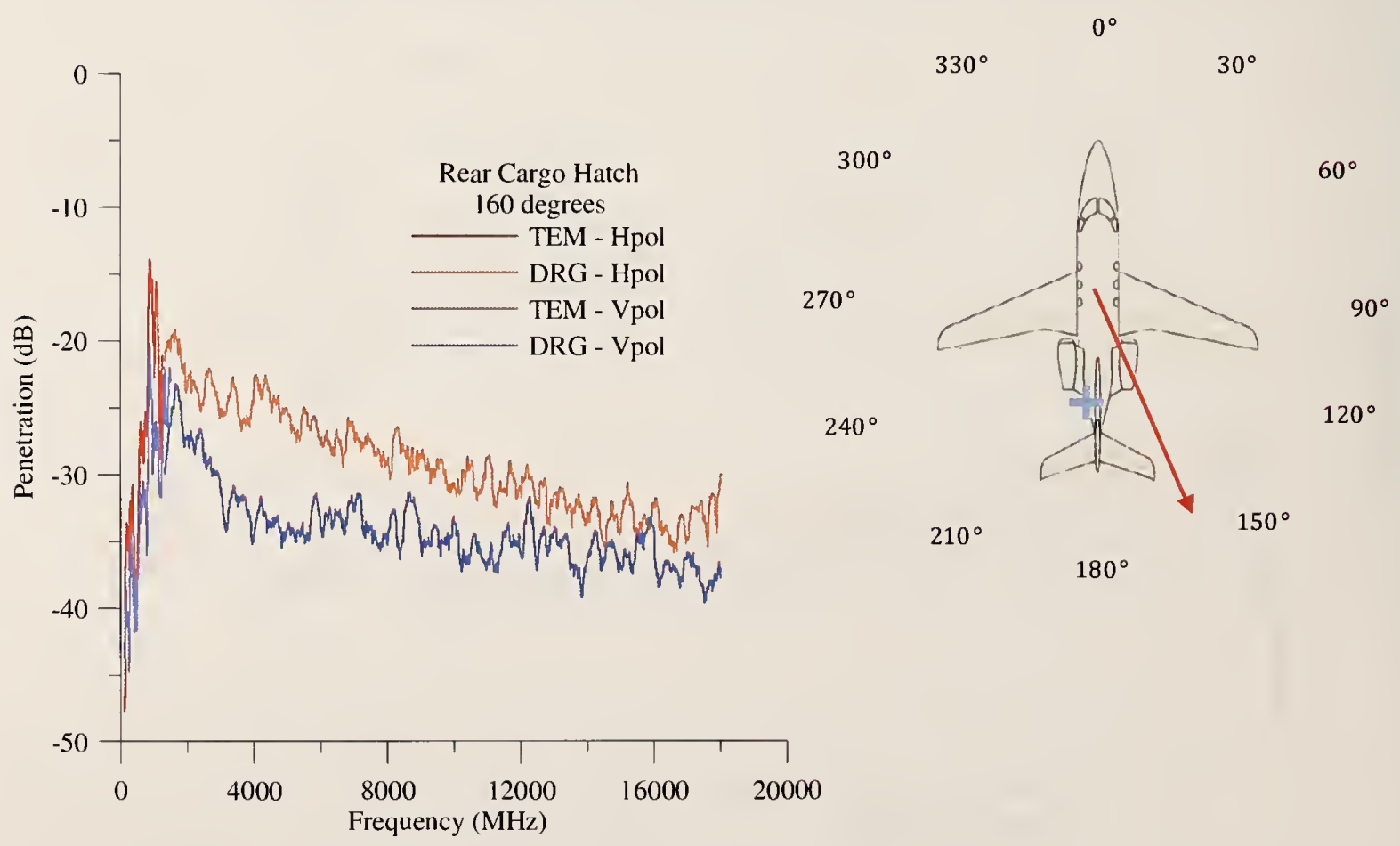

Figure 89. Penetration for all antennas and polarizations for the transmitting tower placed at the $160^{\circ}$ position and the receiving antennas placed in the rear cargo hatch. 


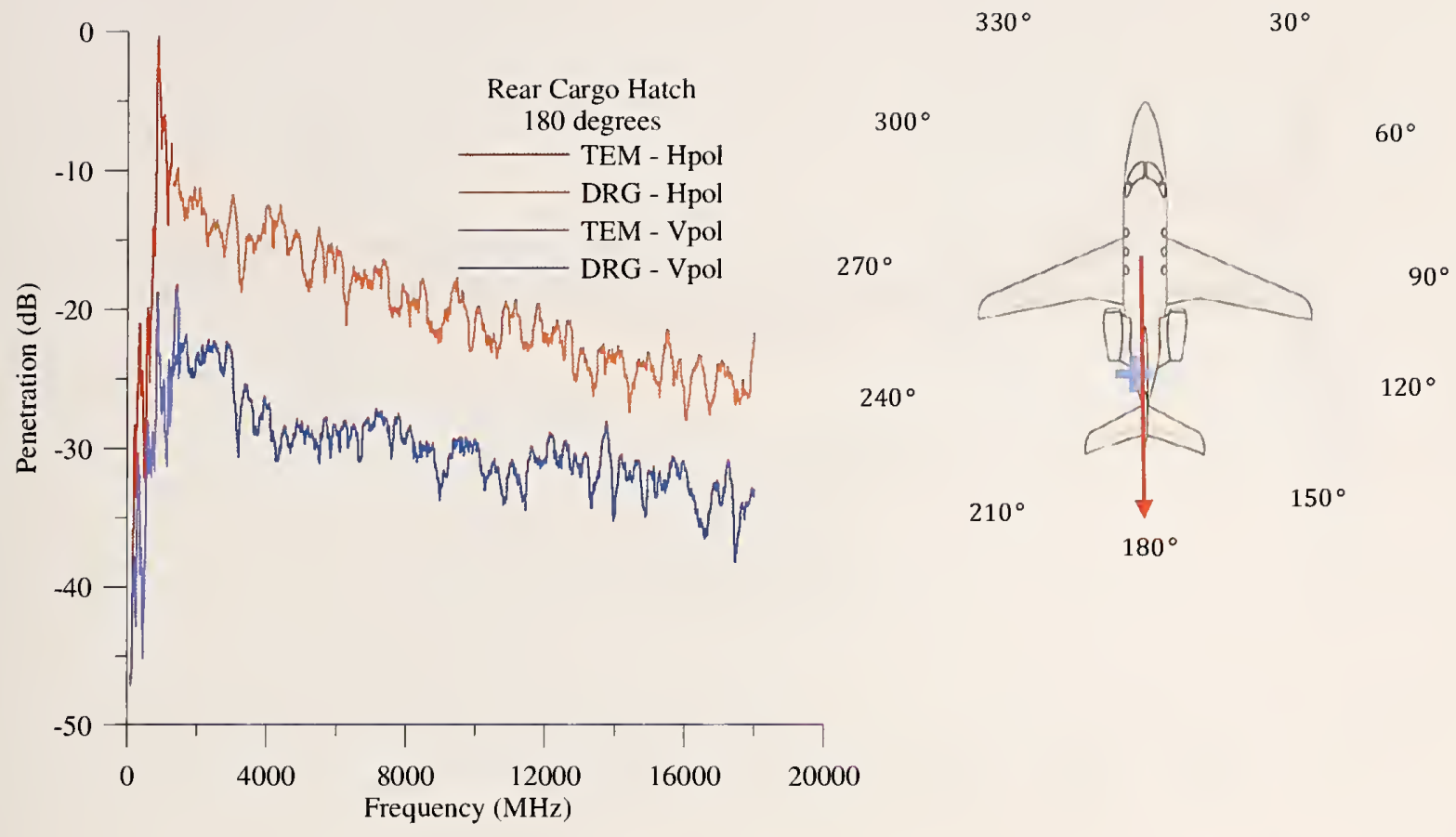

Figure 90. Penetration for all antennas and polarizations for the transmitting tower placed at the $180^{\circ}$ position and the receiving antennas placed in the rear cargo hatch.

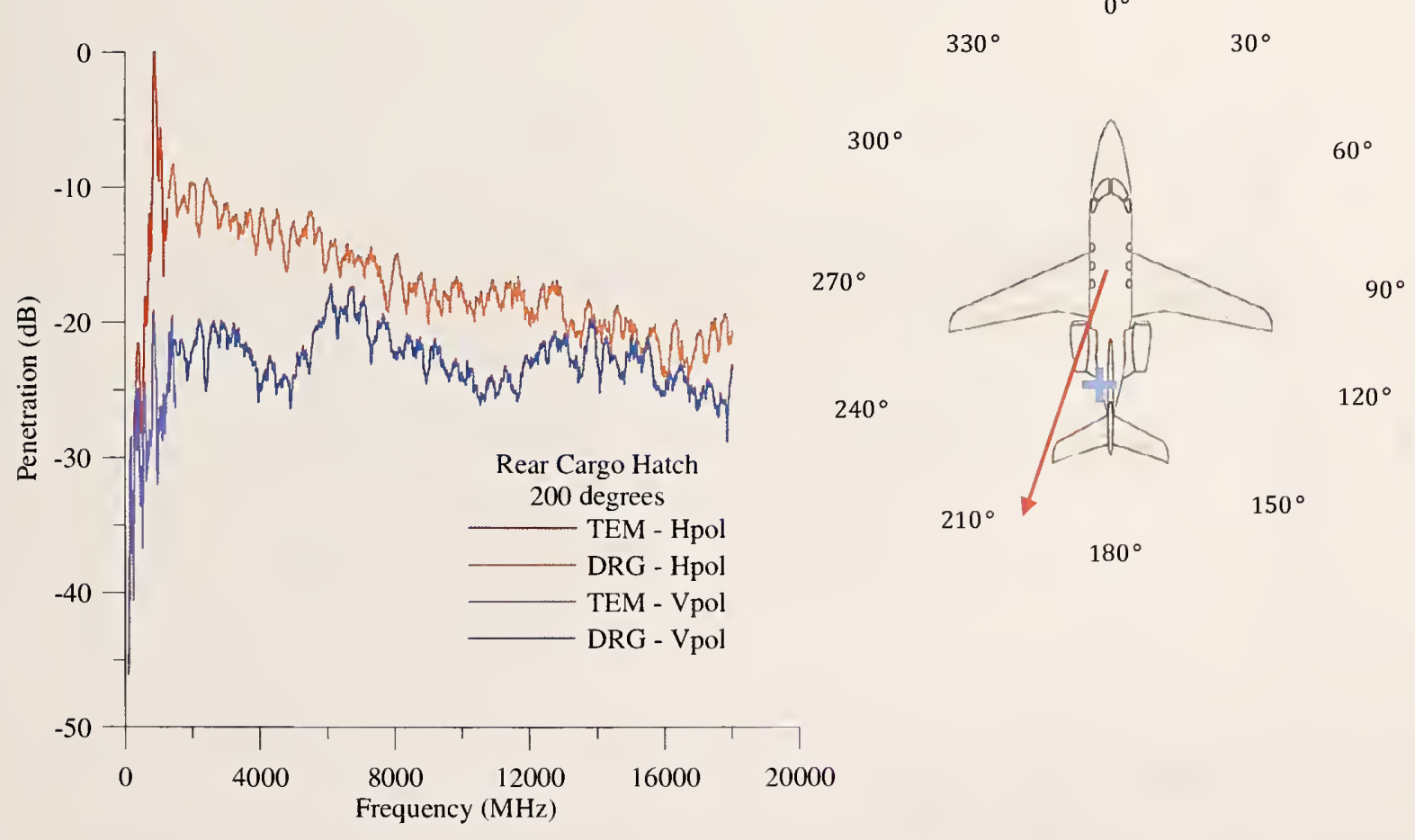

Figure 91. Penetration for all antennas and polarizations for the transmitting tower placed at the $200^{\circ}$ position and the receiving antennas placed in the rear cargo hatch. 


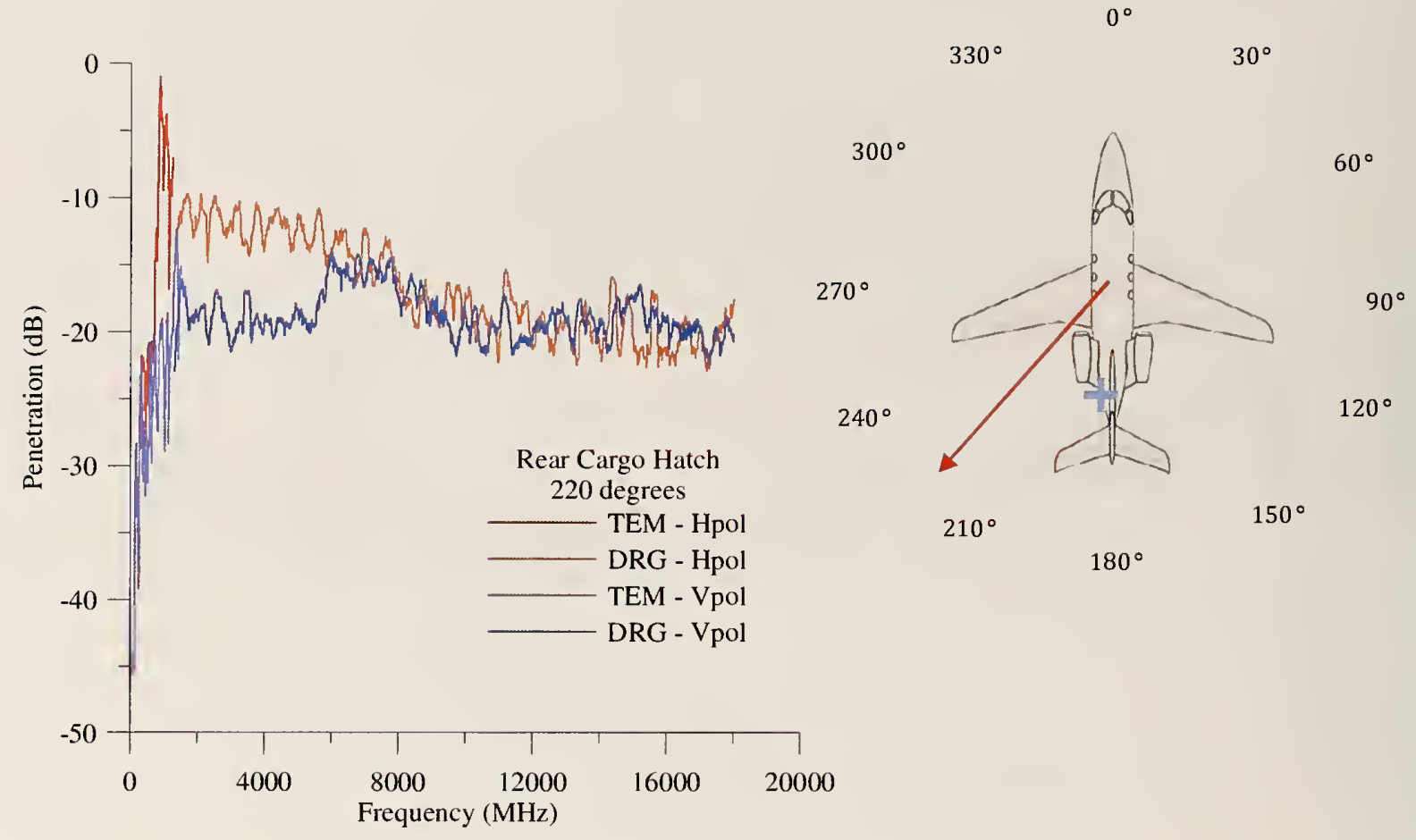

Figure 92. Penetration for all antennas and polarizations for the transmitting tower placed at the $220^{\circ}$ position and the receiving antennas placed in the rear cargo hatch.

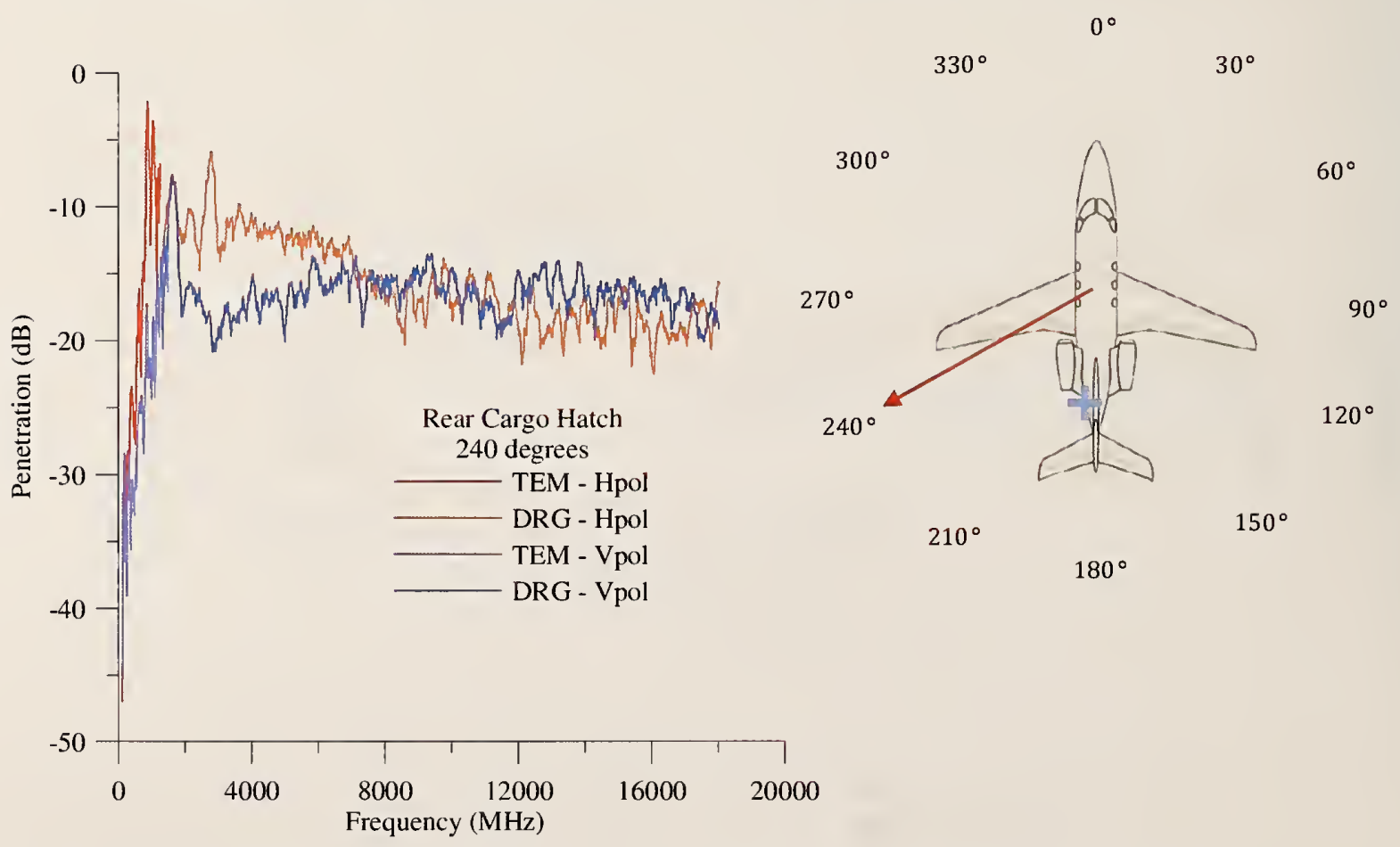

Figure 93. Penetration for all antennas and polarizations for the transmitting tower placed at the $240^{\circ}$ position and the receiving antennas placed in the rear cargo hatch. 


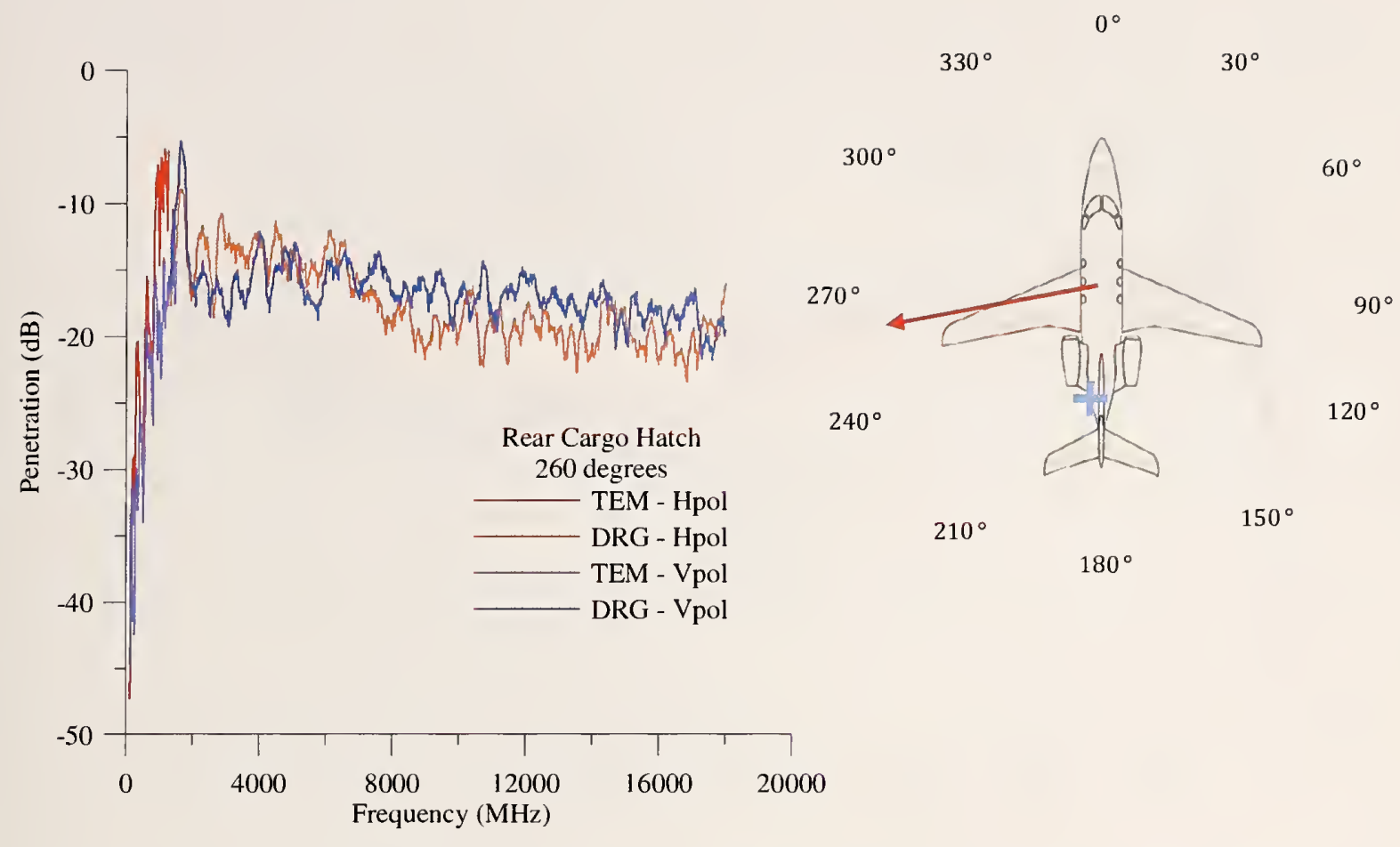

Figure 94. Penetration for all antennas and polarizations for the transmitting tower placed at the $260^{\circ}$ position and the receiving antennas placed in the rear cargo hatch.

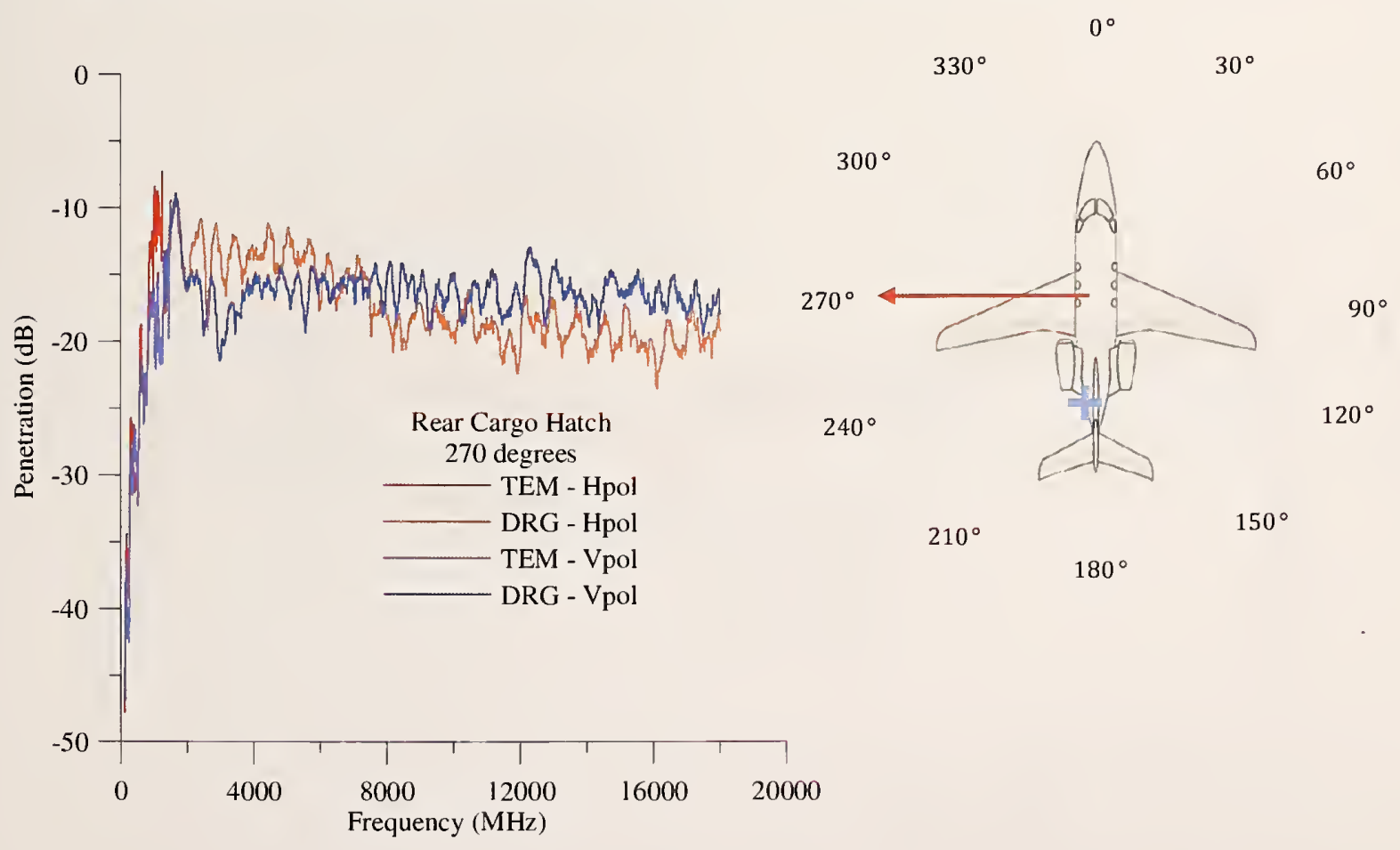

Figure 95. Penetration for all antennas and polarizations for the transmitting tower placed at the $270^{\circ}$ position and the receiving antennas placed in the rear cargo hatch. 


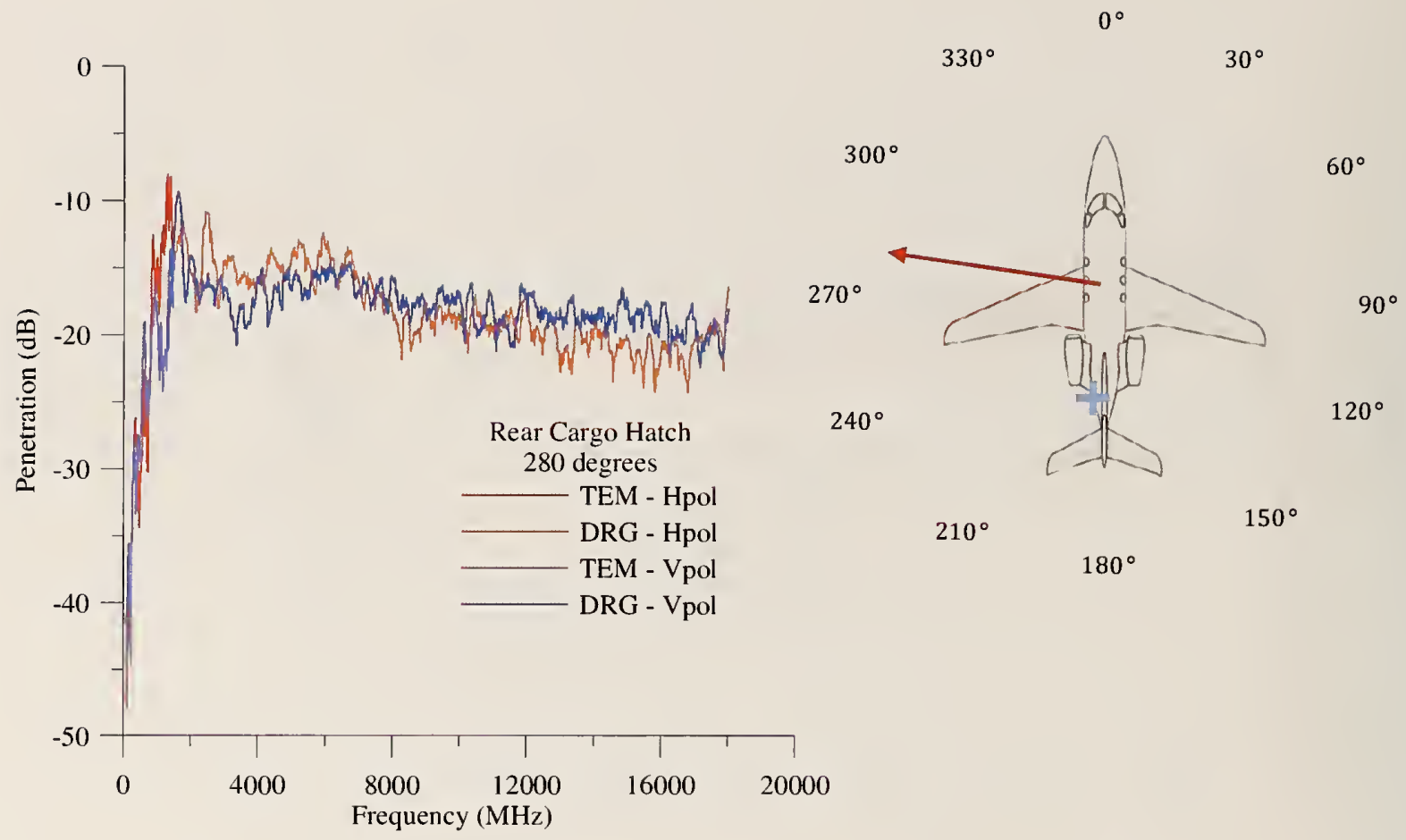

Figure 96. Penetration for all antennas and polarizations for the transmitting tower placed at the $280^{\circ}$ position and the receiving antennas placed in the rear cargo hatch.

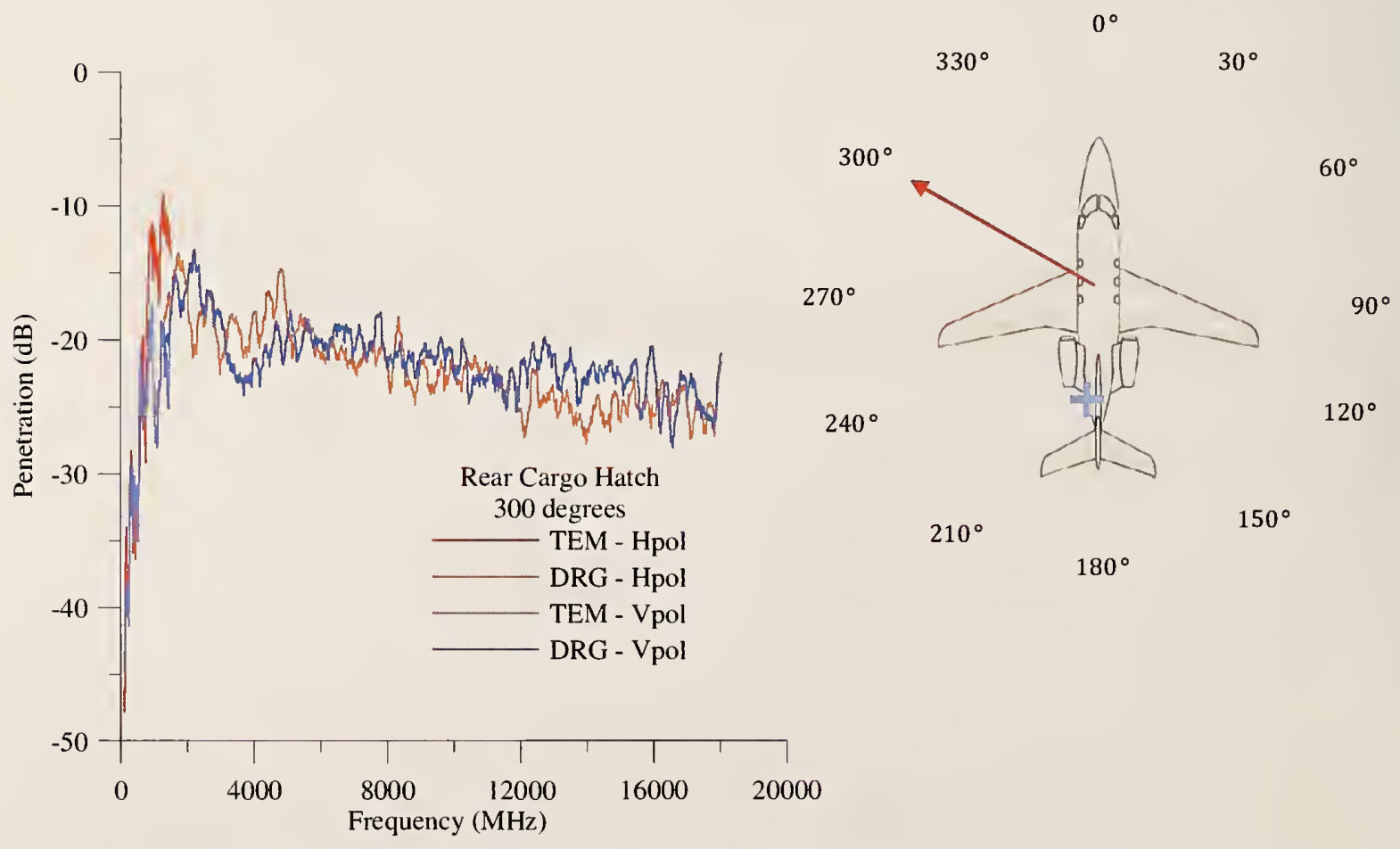

Figure 97. Penetration for all antennas and polarizations for the transmitting tower placed at the $300^{\circ}$ position and the receiving antennas placed in the rear cargo hatch. 


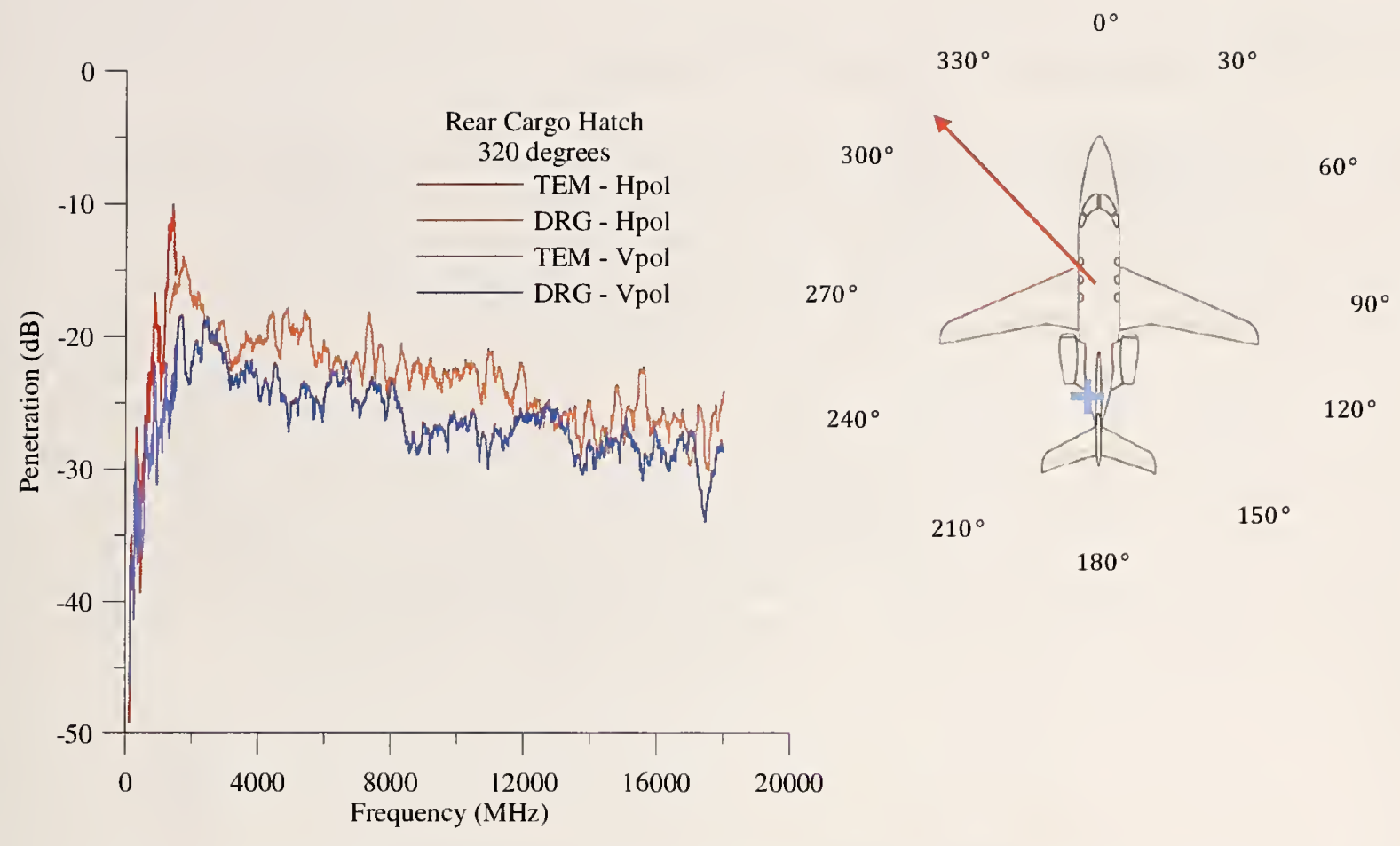

Figure 98. Penetration for all antennas and polarizations for the transmitting tower placed at the $320^{\circ}$ position and the receiving antennas placed in the rear cargo hatch.

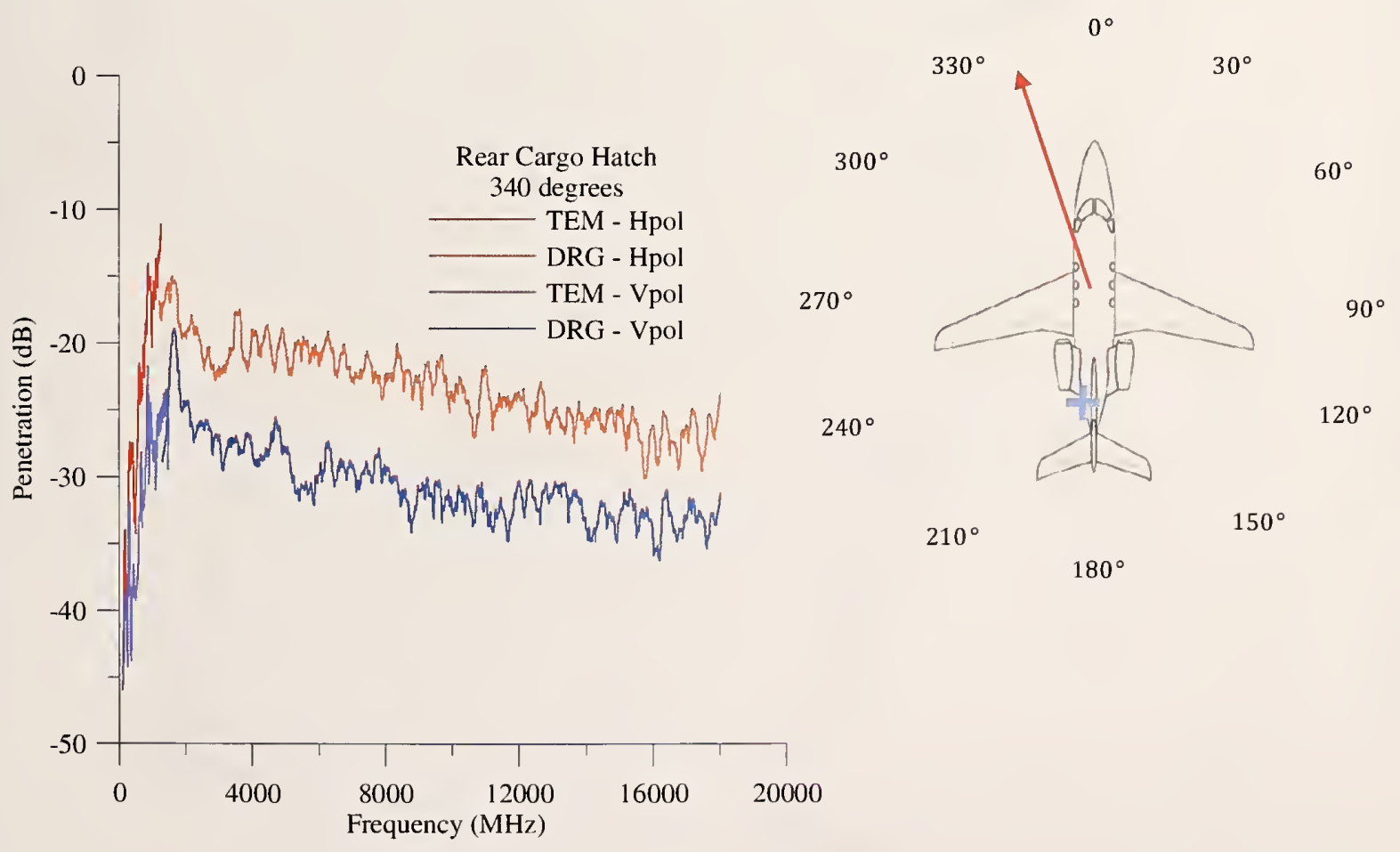

Figure 99. Penetration for all antennas and polarizations for the transmitting tower placed at the $340^{\circ}$ position and the receiving antennas placed in the rear cargo hatch. 


\subsection{HIRF Positions for Flight Deck Penetration}

These tests are standard measurements taken for HIRF evaluation of airframe shielding and vulnerability as discussed in reference [2]. Five measurements were taken around the flight deck of the aircraft to investigate shielding vulnerabilities. In our measurements, we offset the transmitting antenna approximately $15 \mathrm{~m}$ from the front wheel of the aircraft. There is no required offset from the flight deck windows for testing, but there are requirements for angular positions. These are at $270^{\circ}, 315^{\circ}, 0^{\circ}, 45^{\circ}$, and $90^{\circ}$ around the flight deck windows. Figure 100 shows a diagram of these measurement positions and their associated graphs. We see that the penetration has a maximum value of approximately $-5 \mathrm{~dB}$ at frequencies below $4000 \mathrm{MHz}$ for the horizontal and vertical polarizations. The minimum penetration values that occur at the higher frequencies are between $-15 \mathrm{~dB}$ and $-20 \mathrm{~dB}$. At an angle of $0^{\circ}$, we see three sharp resonances at approximately $1000 \mathrm{MHz}, 2000 \mathrm{MHz}$, and $4000 \mathrm{MHz}$ for the TEM and DRG antennas in the horizontal polarization. The penetration levels remain similar for each HIRF position. The separate plots are given in Figures 101 to 105. 

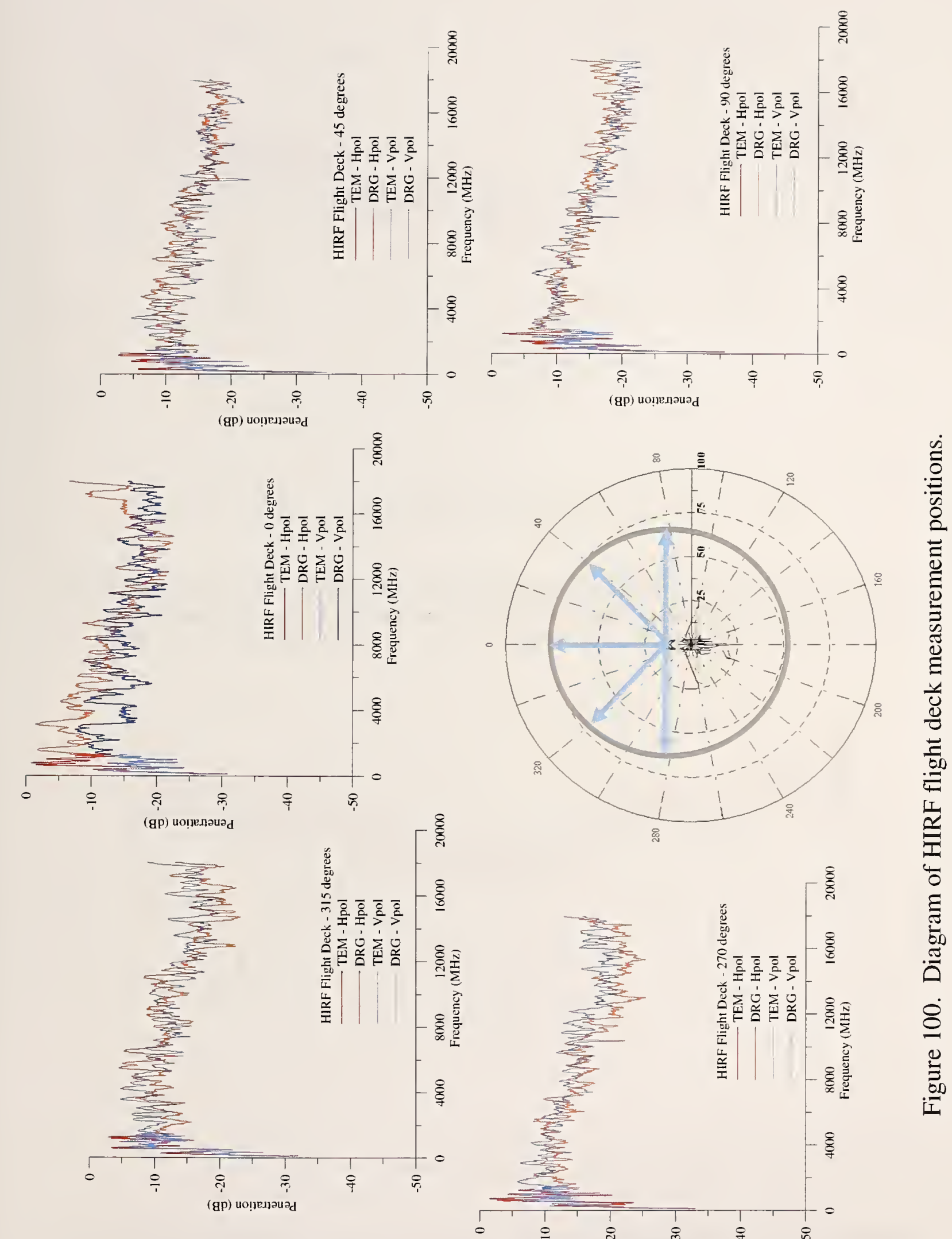


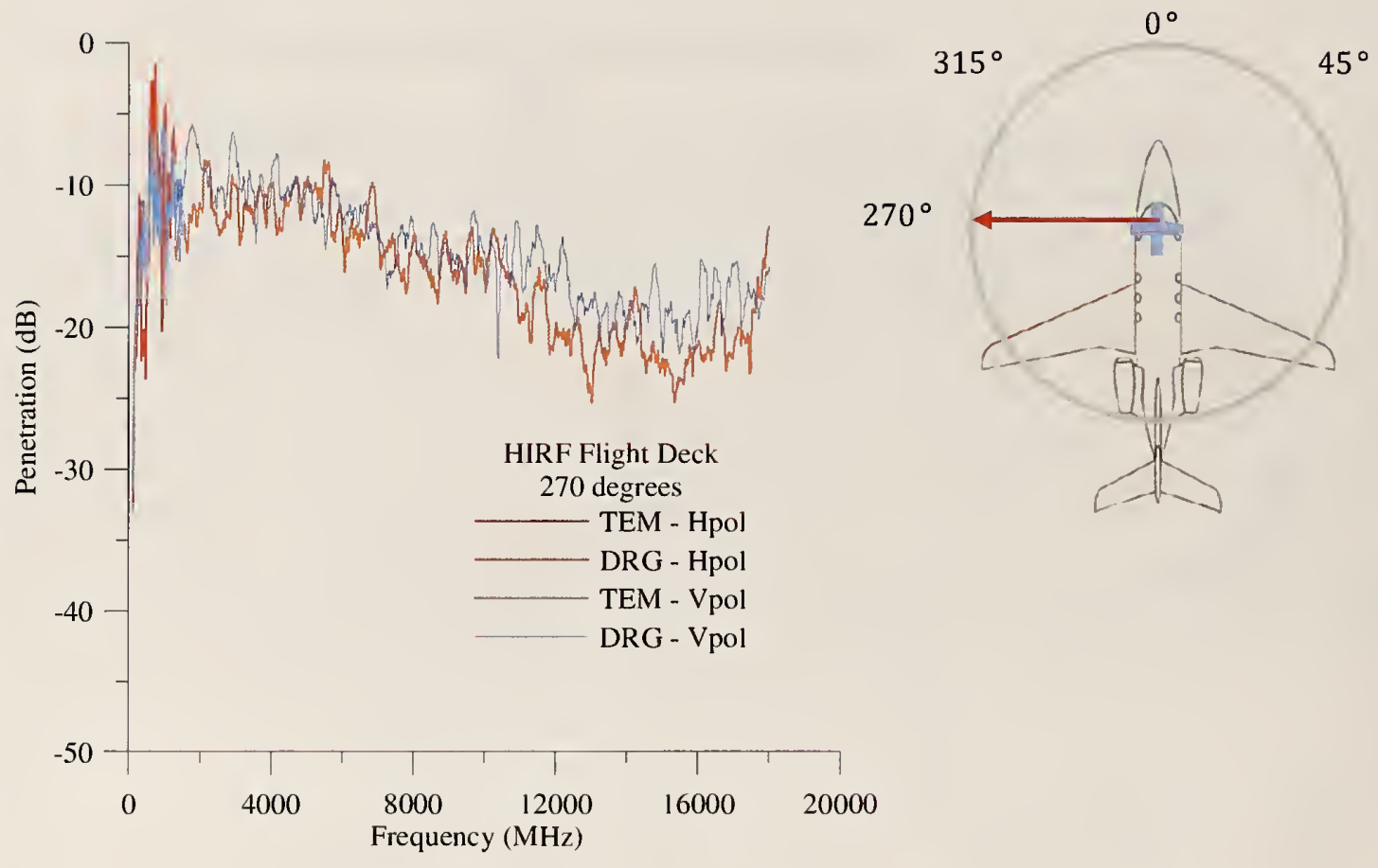

$90^{\circ}$

Figure 101. HIRF Penetration data for all antennas and polarizations for the transmitting tower placed at the $270^{\circ}$ position, at a distance of $15 \mathrm{~m}$ centered on the front landing gear and the receiving antennas placed in the flight deck.

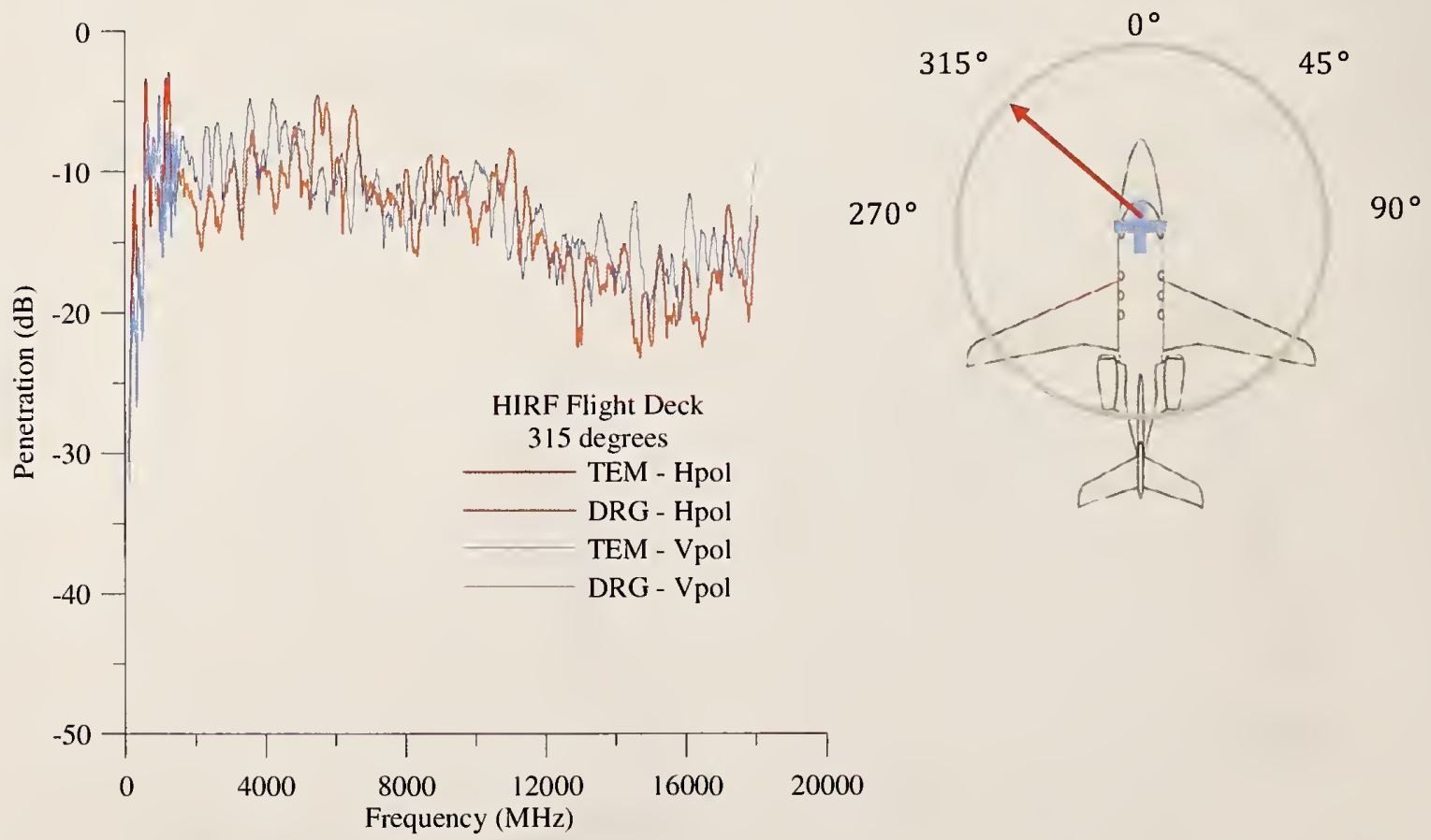

Figure 102. HIRF Penetration data for all antennas and polarizations for the transmitting tower placed at the $315^{\circ}$ position, at a distance of $15 \mathrm{~m}$ centered on the front landing gear and the receiving antennas placed in the flight deck. 

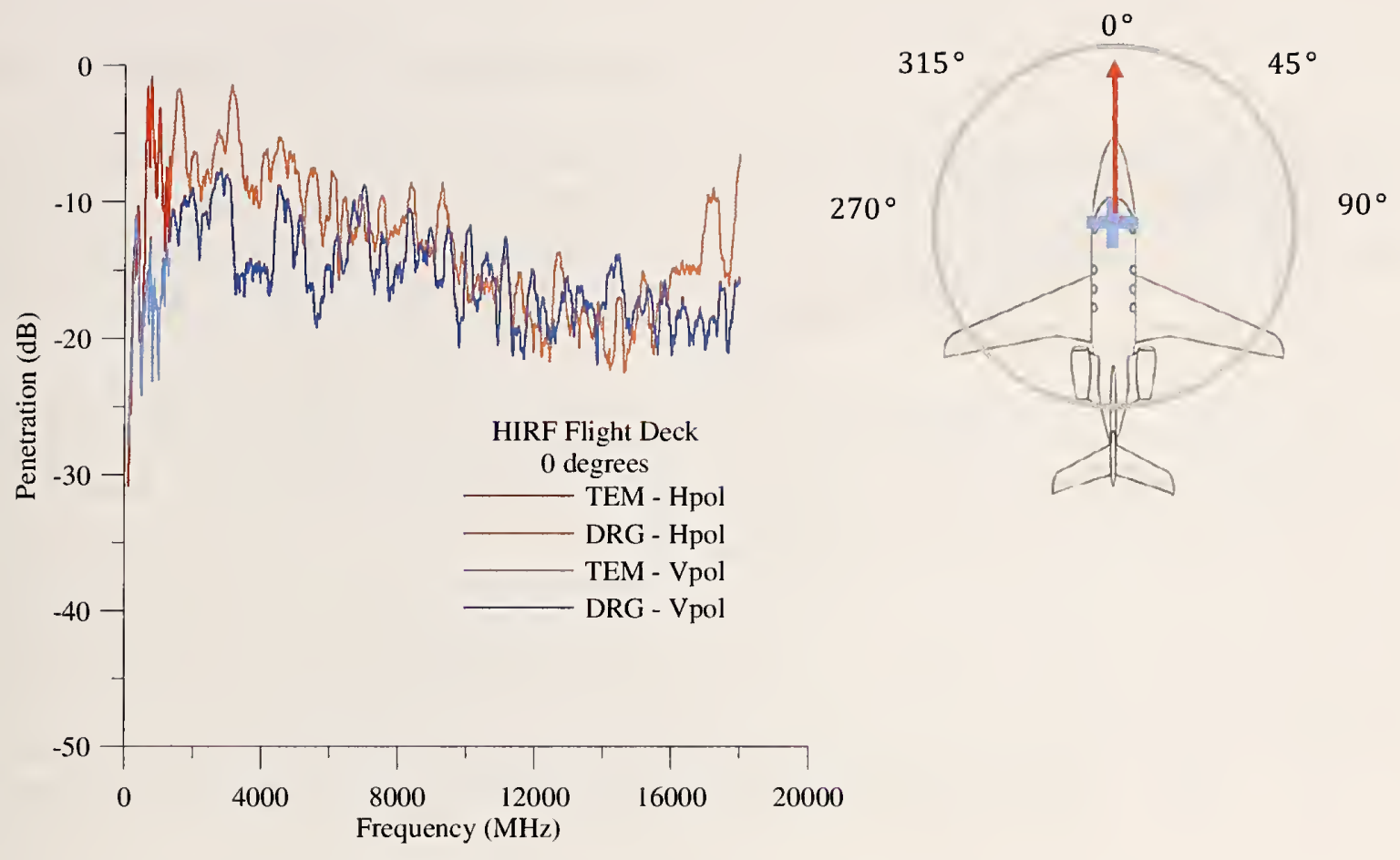

Figure 103. HIRF Penetration data for all antennas and polarizations for the transmitting tower placed at the $0^{\circ}$ position, at a distance of $15 \mathrm{~m}$ centered on the front landing gear and the receiving antennas placed in the flight deck.

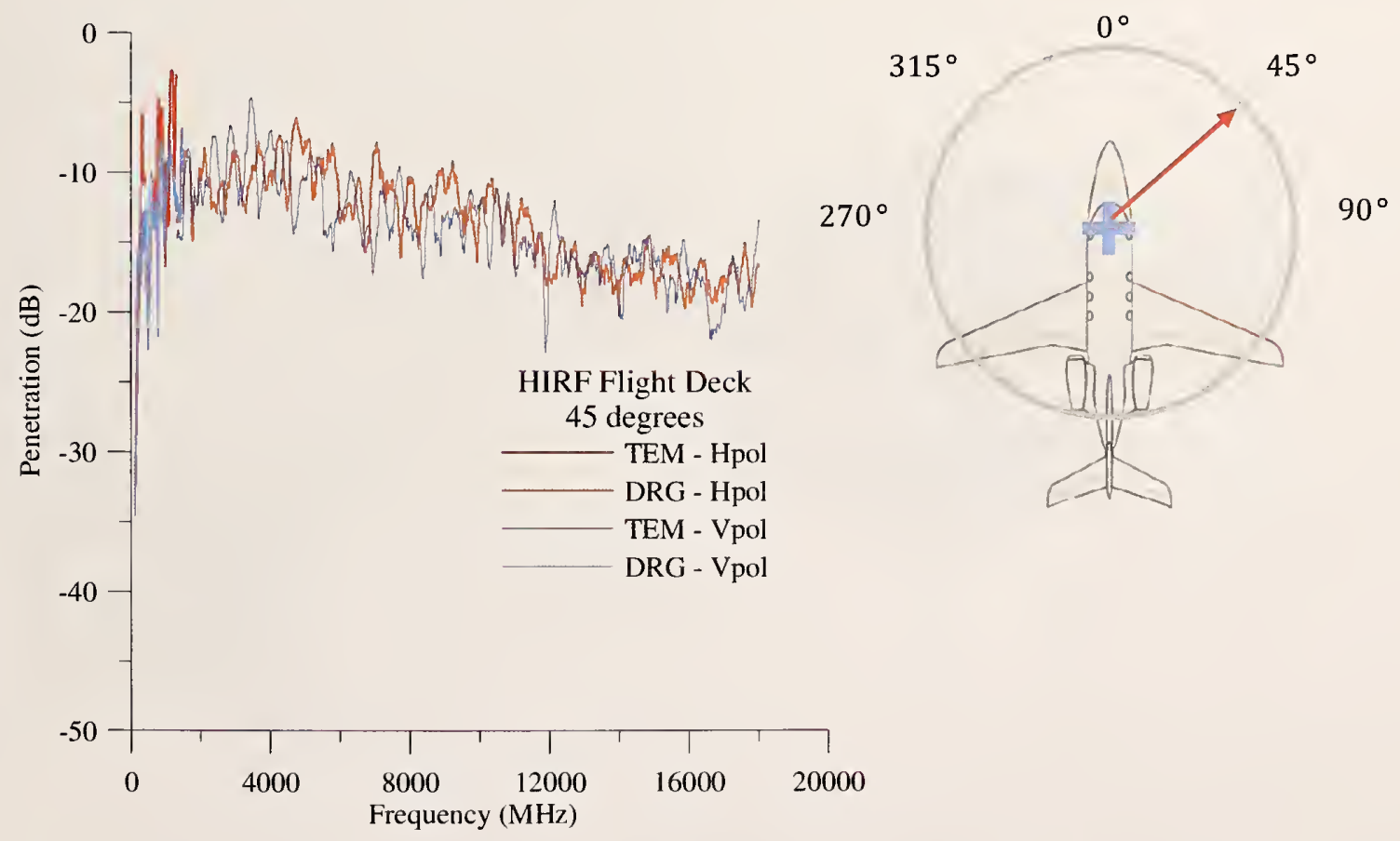

Figure 104. HIRF Penetration data for all antennas and polarizations for the transmitting tower placed at the $45^{\circ}$ position, at a distance of $15 \mathrm{~m}$ centered on the front landing gear and the receiving antennas placed in the flight deck. 


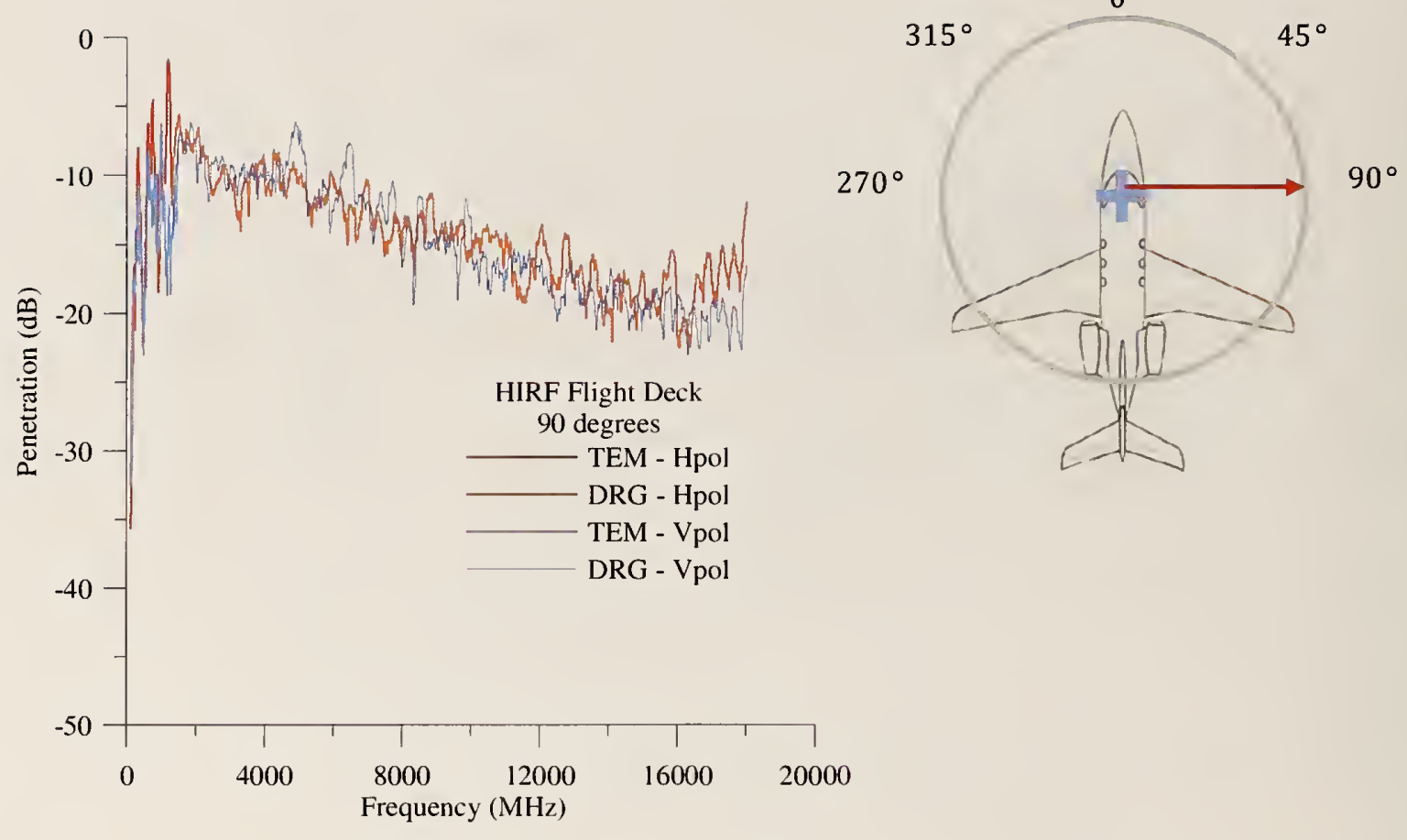

Figure 105. HIRF Penetration data for all antennas and polarizations for the transmitting tower placed at the $90^{\circ}$ position, at a distance of $15 \mathrm{~m}$ centered on the front landing gear and the receiving antennas placed in the flight deck. 


\subsection{Internal Coupling Measurements}

Internal coupling measurements can tell us a lot about the propagation environment within the airframe. They can tell us whether fields are uniform and whether they decay rapidly or more slowly over time. They can also tell us how well the fields propagate within the airframe and whether they can easily reach critical components inside the airframe.

NIST conducted several internal coupling measurements with each antenna to determine the decay time and the quality factor of the internal airframe volume. Because the cabin was particularly small, the combinations of DRG and TEM antennas were measured separately. We also had to configure the system differently than for the Bombardier and Boeing 737-200 so that the network analyzer could be left on the outside of the aircraft, because there was no room for it internally. This new configuration is shown in Figure 106. The individual configurations for the DRG antennas are shown in Figures 107 to 111 and for the TEM antennas in Figures 112 to 115. The chosen configurations were meant to physically stir the fields inside the aircraft as much as possible. The red polygons represent the transmitting and receiving DRG antennas, and the blue rectangles represent the transmitting and receiving TEM horn antennas.

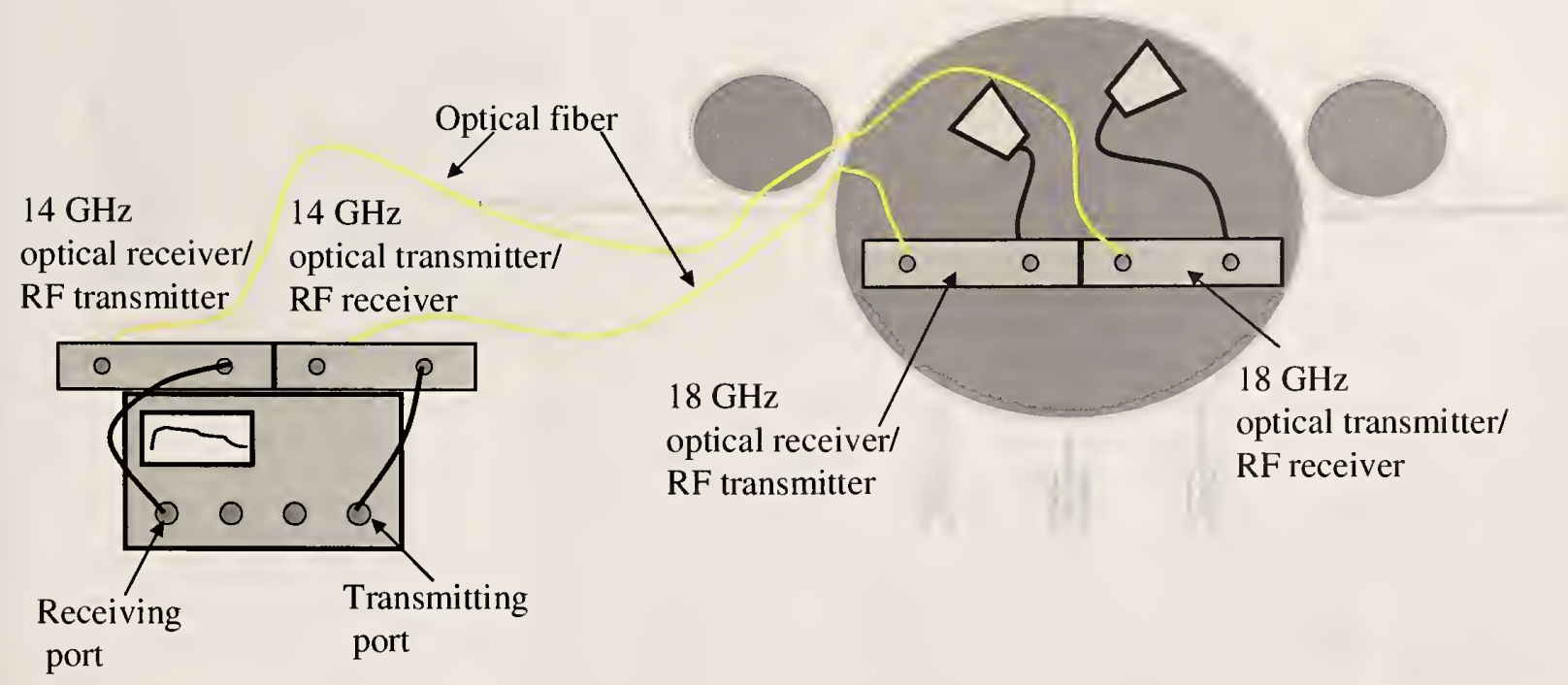

Figure 106. Measurement setup for internal configurations. 


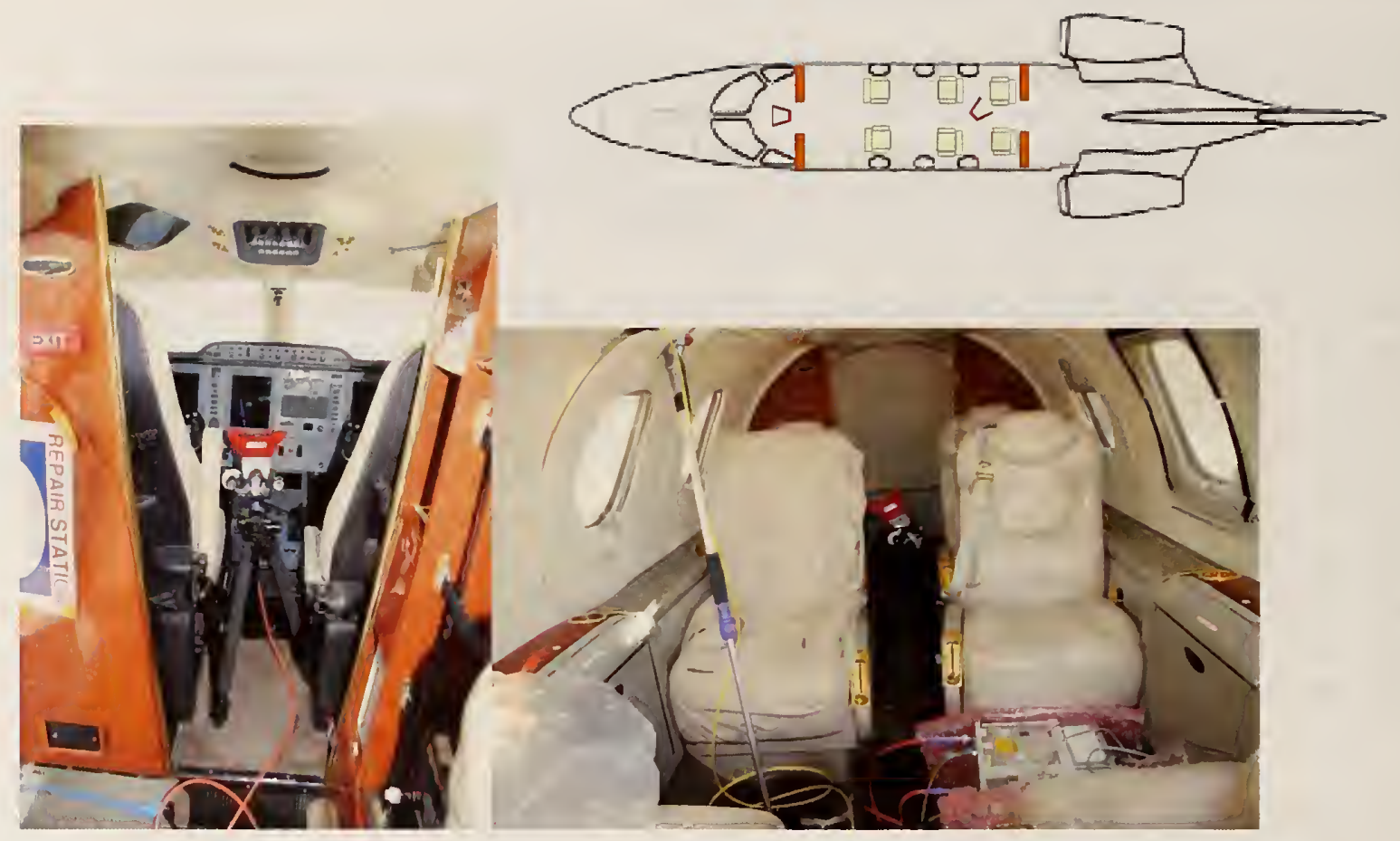

Figure 107. Layout for DRG configuration 1 measurements. One antenna points toward the front of the flight deck. The second antenna points toward the rear of the aircraft.

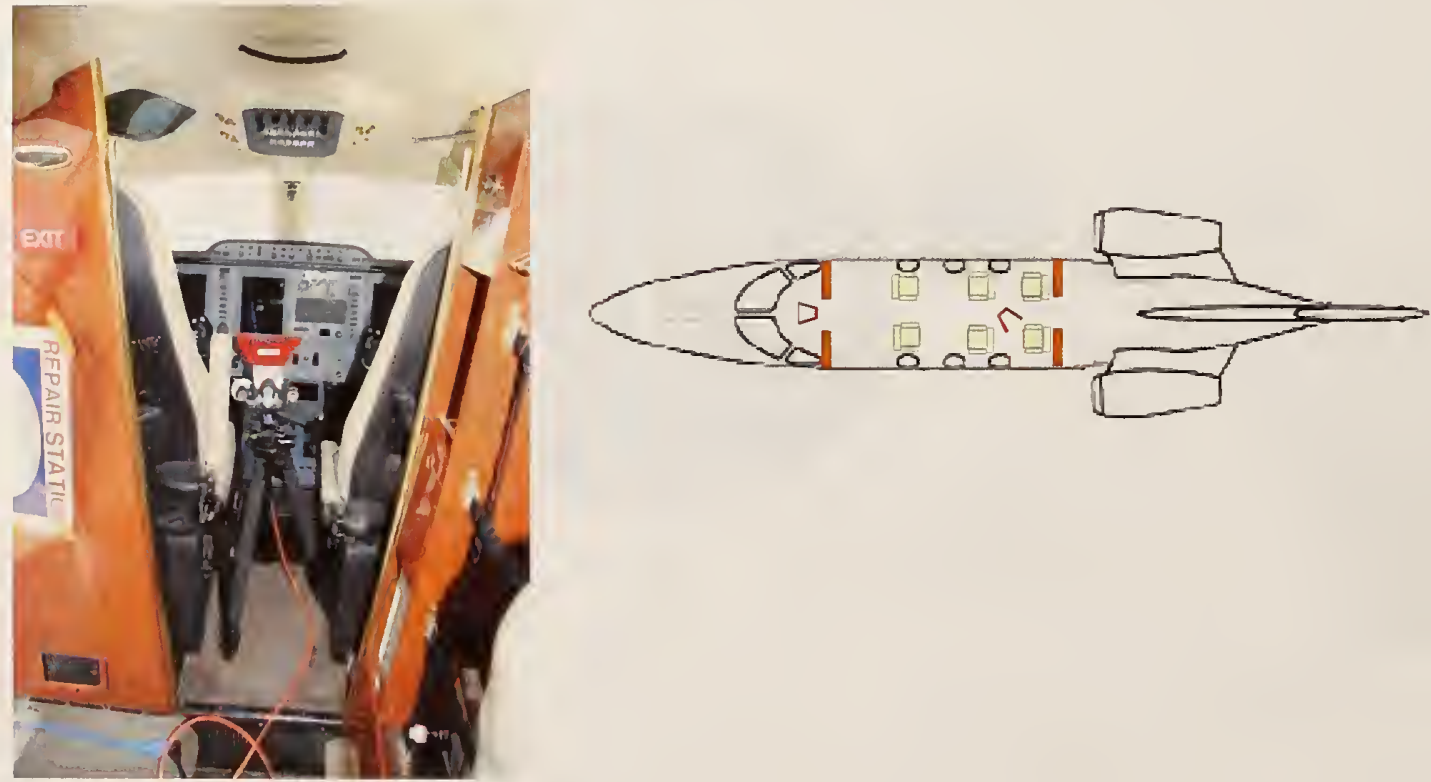

Figure 108. Layout for DRG configuration 2 measurements. One antenna points toward the front of the flight deck. The second antenna points into the last righthand seat. 

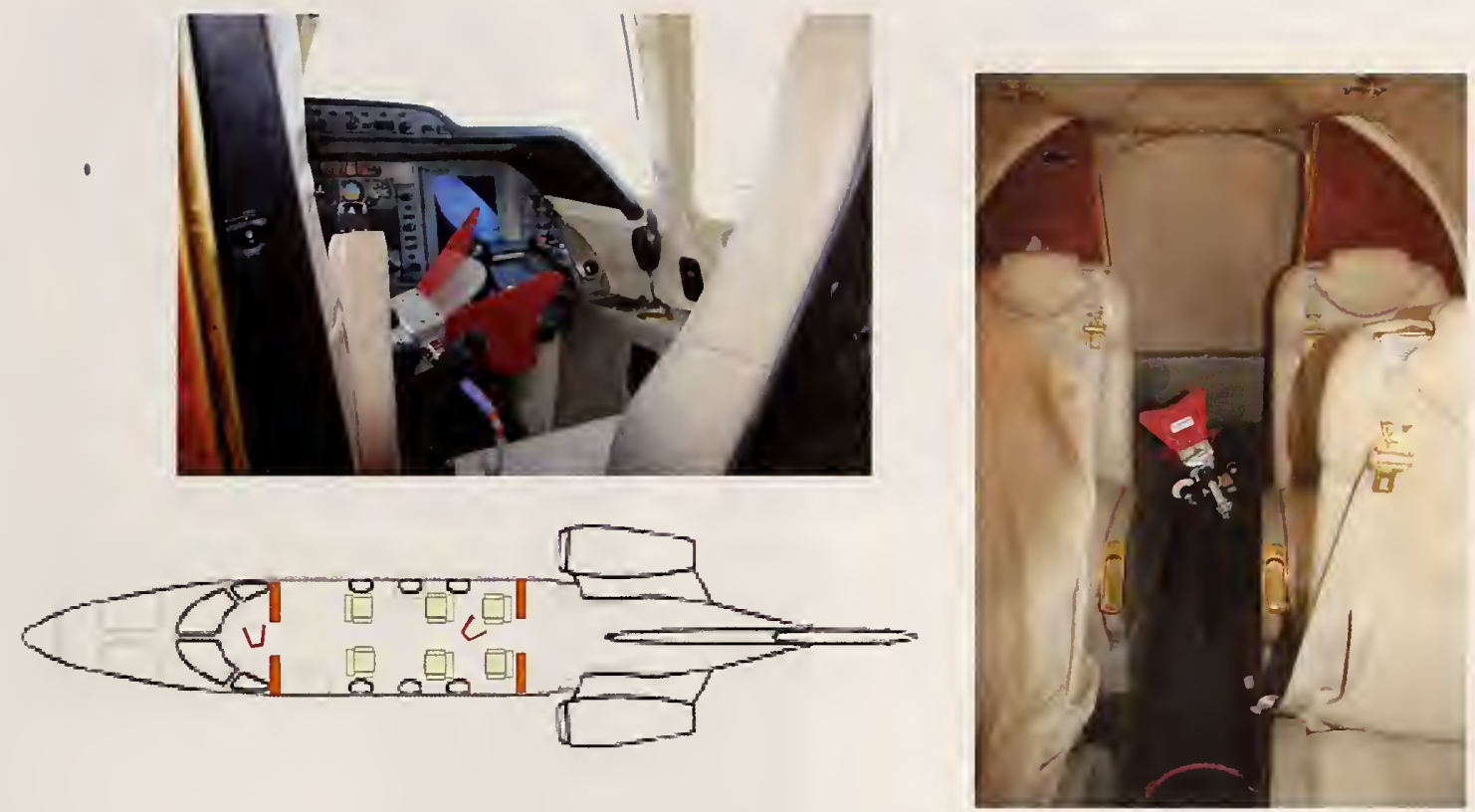

Figure 109. Layout for DRG configuration 3 measurements. One antenna points out of the left window in the flight deck. The second antenna points into the last lefthand seat.

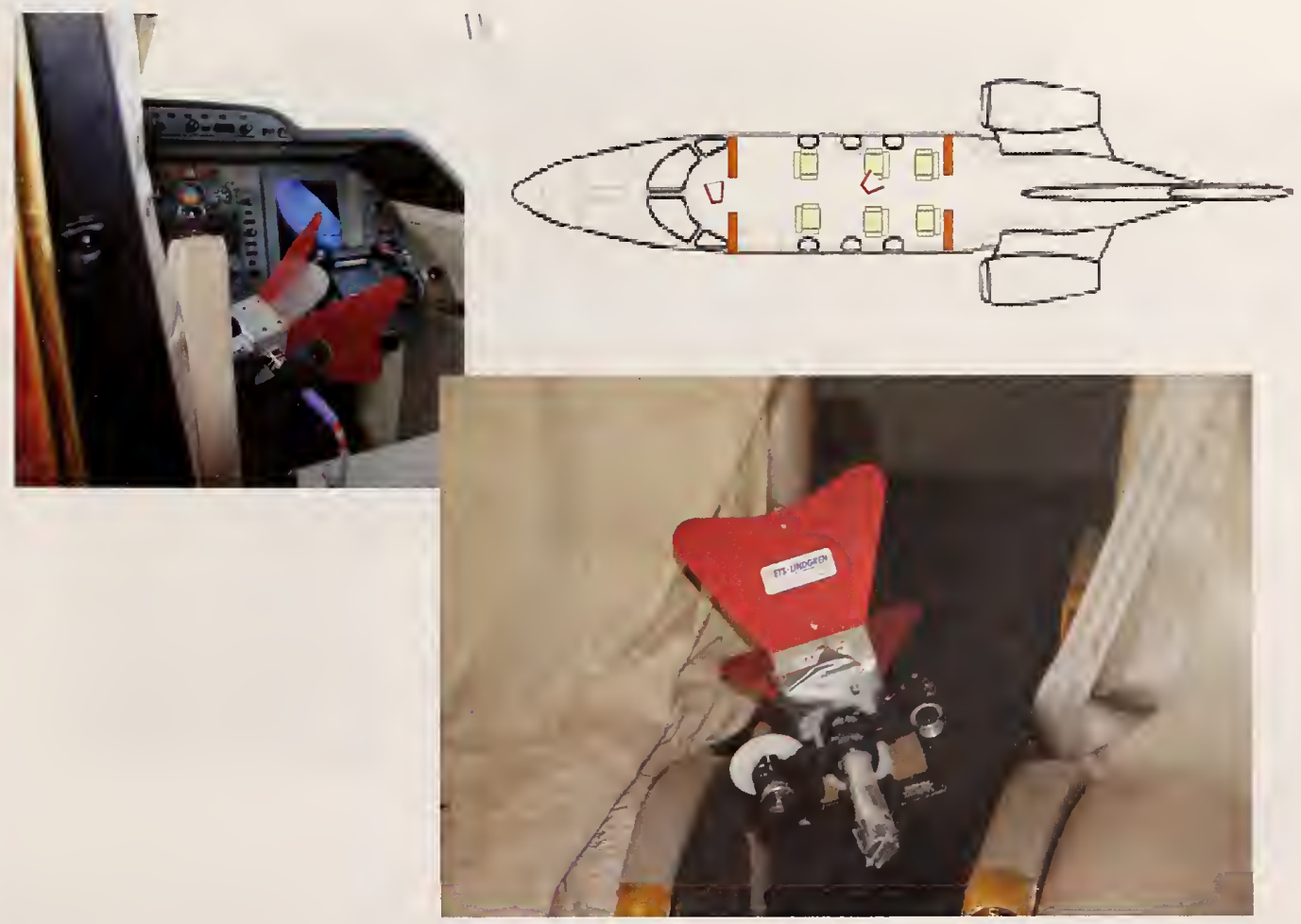

Figure 110. Layout for DRG configuration 4 measurements. One antenna points out of the left window in the flight deck. The second antenna points into the middle lefthand seat. 


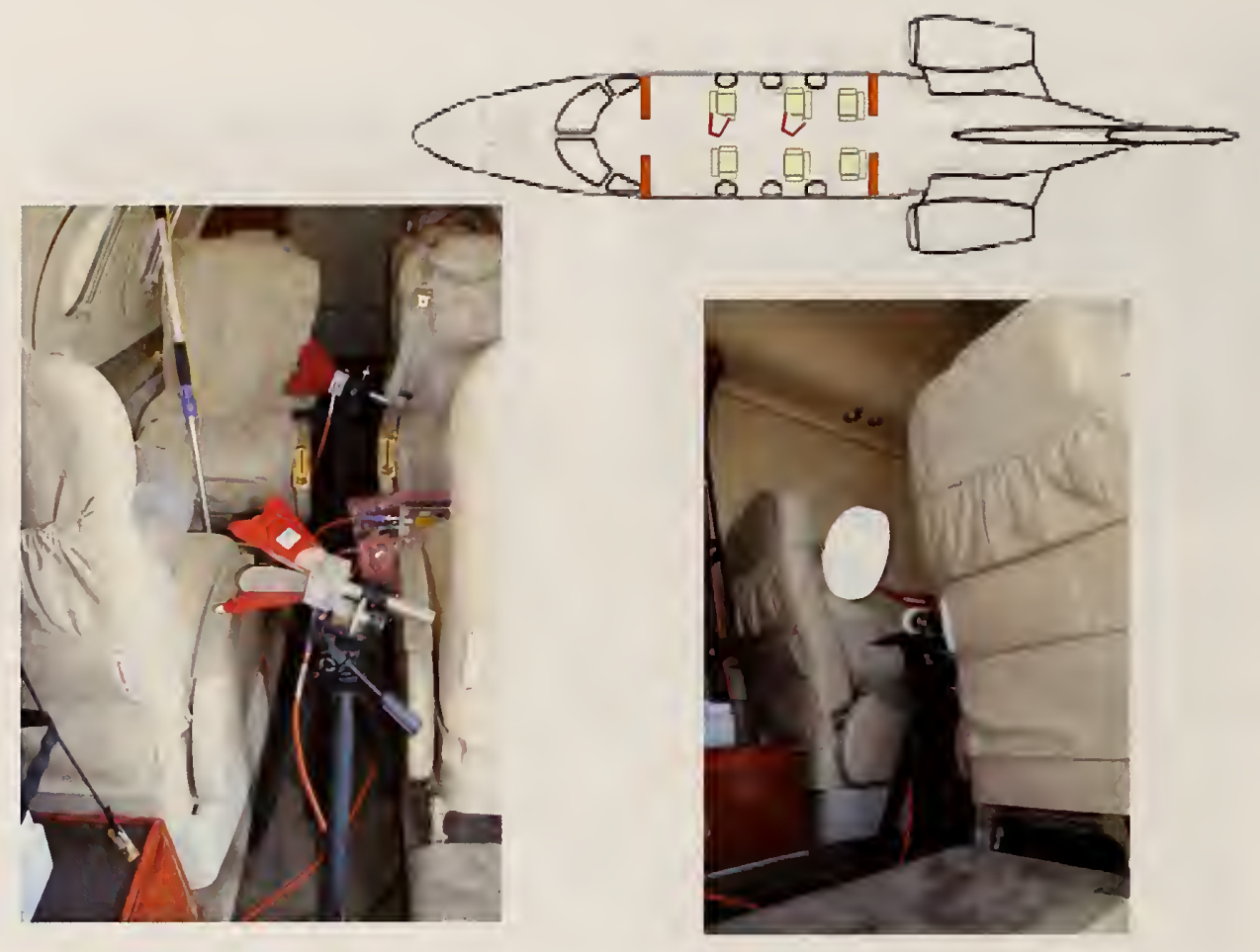

Figure 111. Layout for DRG configuration 5 measurements. One antenna points toward the first, lefthand window in the passenger cabin. The second antenna points into the middle lefthand seat.

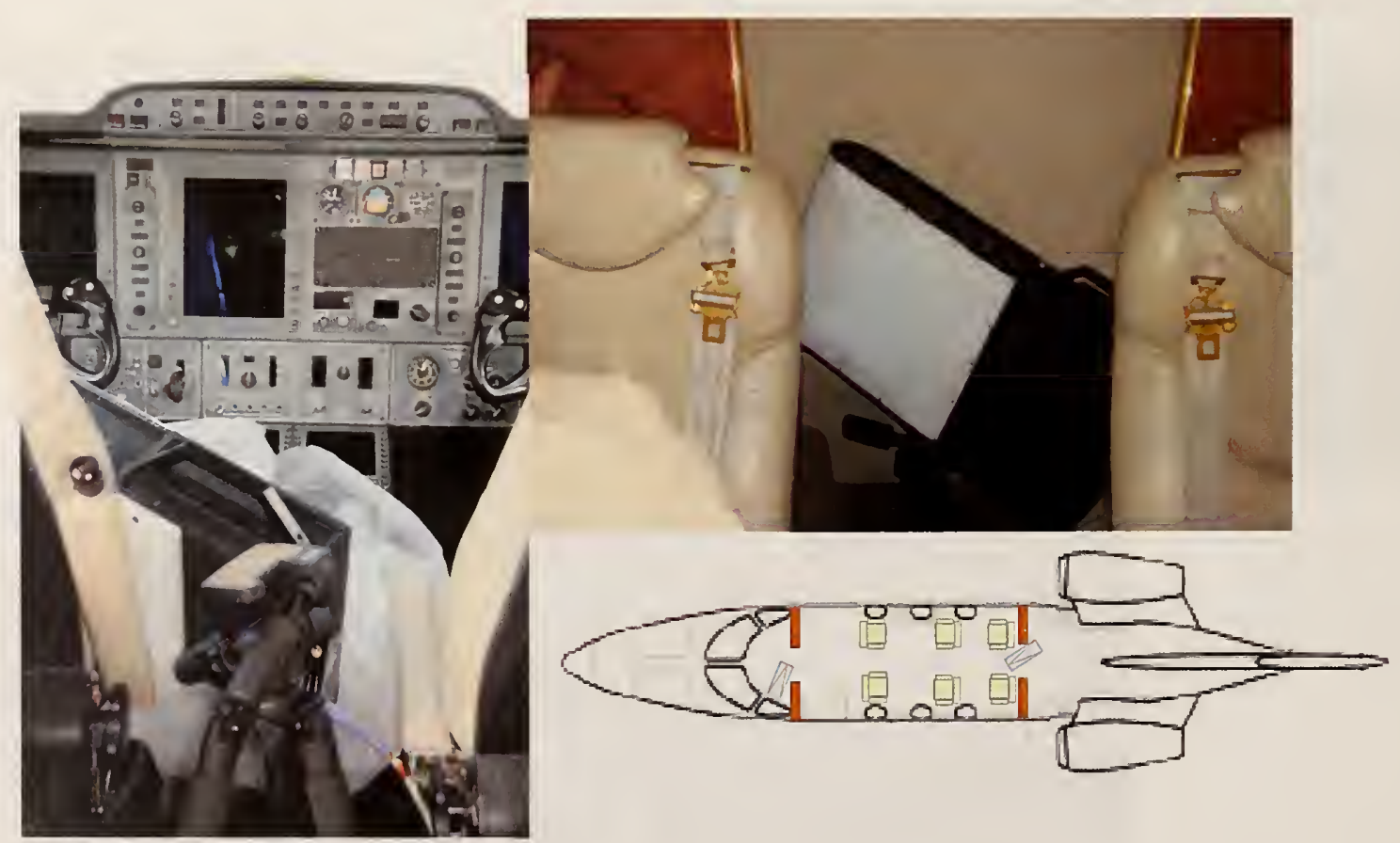

Figure 112. Layout for TEM configuration 1 measurements. One antenna points out the righthand window of the flight deck. The second antenna points toward the left rear of the aircraft. 


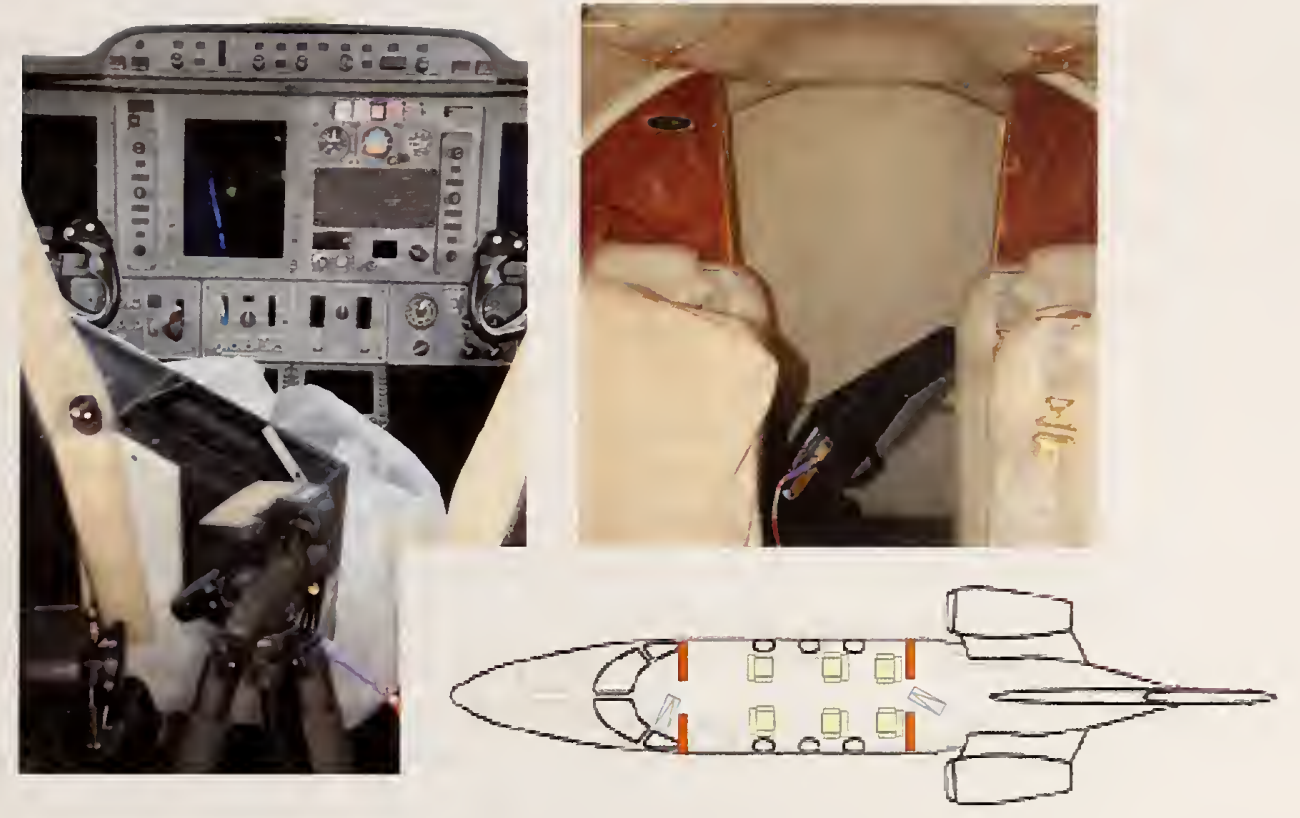

Figure 113. Layout for TEM configuration 2 measurements. One antenna points out the righthand window of the flight deck. The second antenna points toward the right rear of the aircraft.

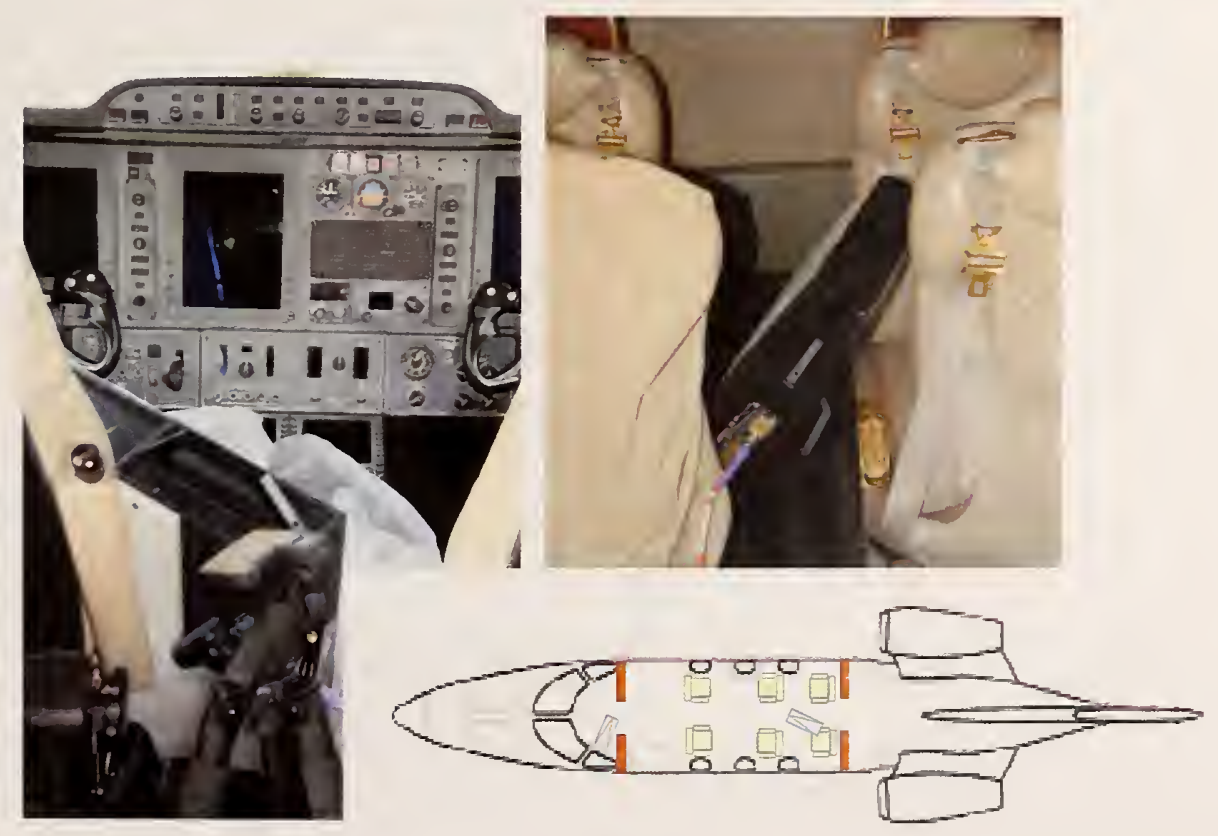

Figure 114. Layout for TEM configuration 3 measurements. One antenna points out the righthand window of the flight deck. The second antenna points toward the right rear seat. 
We begin by looking at the time decay and quality factor of the airframe for each set of antennas in each configuration. These are shown in Figures 116 to 119 . Time decay is defined as the period needed for the signal power to decay to its $\mathrm{e}^{-1}$ value. The decay over time for the DRG antennas in Figure 116 shows loose clustering behavior from $1 \mathrm{GHz}$ to $16 \mathrm{GHz}$. The variation in decay over time below $8 \mathrm{GHz}$ is anywhere from two nanoseconds to approximately seven nanoseconds. The variation above $8 \mathrm{GHz}$ is from seven nanoseconds to ten nanoseconds. There is no discernable pattern behavior as a function of position. The variation in decay over time for the TEM horn antennas is on the order of a couple of nanoseconds to approximately seven nanoseconds. The trend shows tighter clustering for the DRG antennas at the lower frequencies and for the TEM antennas in the higher frequency range. The quality factor is defined as the radian frequency multiplied by the decay period. The quality factor is much lower for the TEM horn antennas because of the lower frequency range. Figures 120 and 121 are plots showing the combined quality factor for both antennas. In Figure 120, we show the combined quality factor for the DRG configuration 5 and the complementary configuration 4 for the TEM horn antennas. Figure 121 shows both antennas for configuration 1. The two antenna types show good agreement across their common frequency band from $1 \mathrm{GHz}$ to $2 \mathrm{GHz}$. The testing community would like the airframe to approximate a reverberant environment so that certain statistical models can be applied. In a reverberant cavity, we expect the quality factor and time decay to vary little throughout the cavity. Because the quality factor is so low we do not expect a large number of modes that would support a good reverberant environment. However, further statistical analysis would have to be completed in order to determine that the cavity itself is not reverberant.

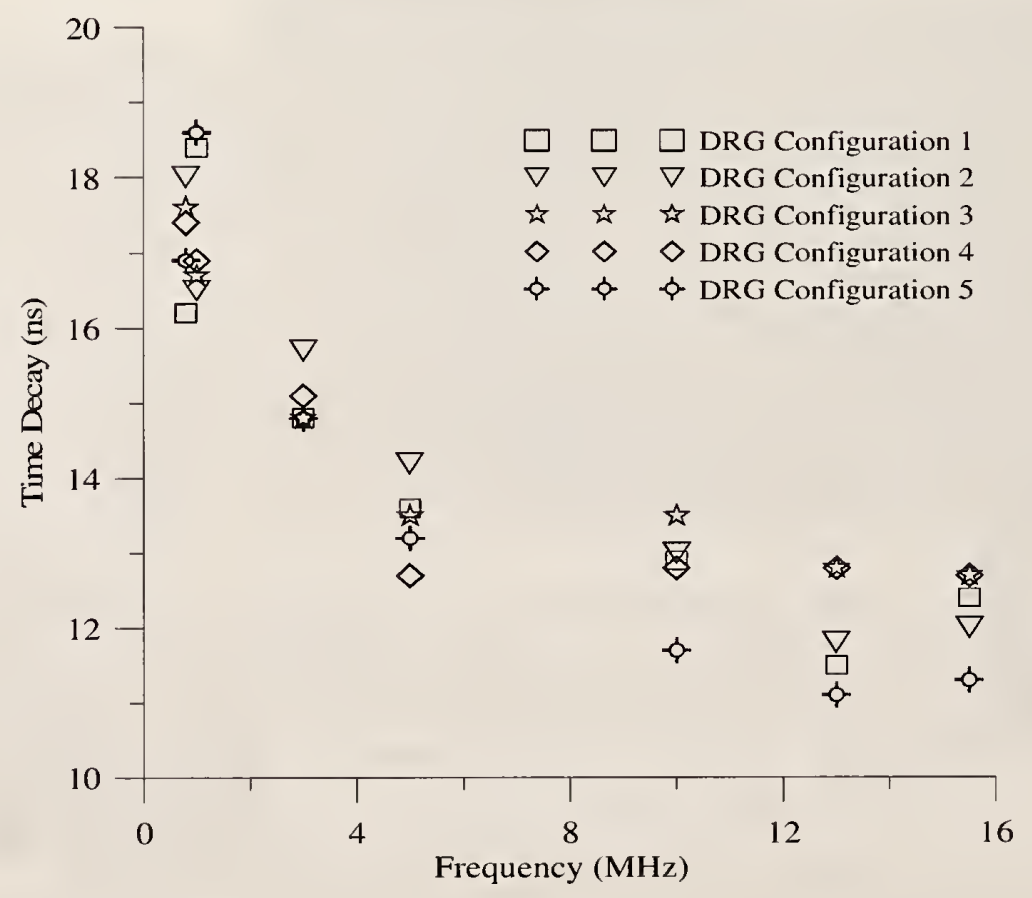

Figure 116. Time decay for DRG antennas in all five internal configurations. 


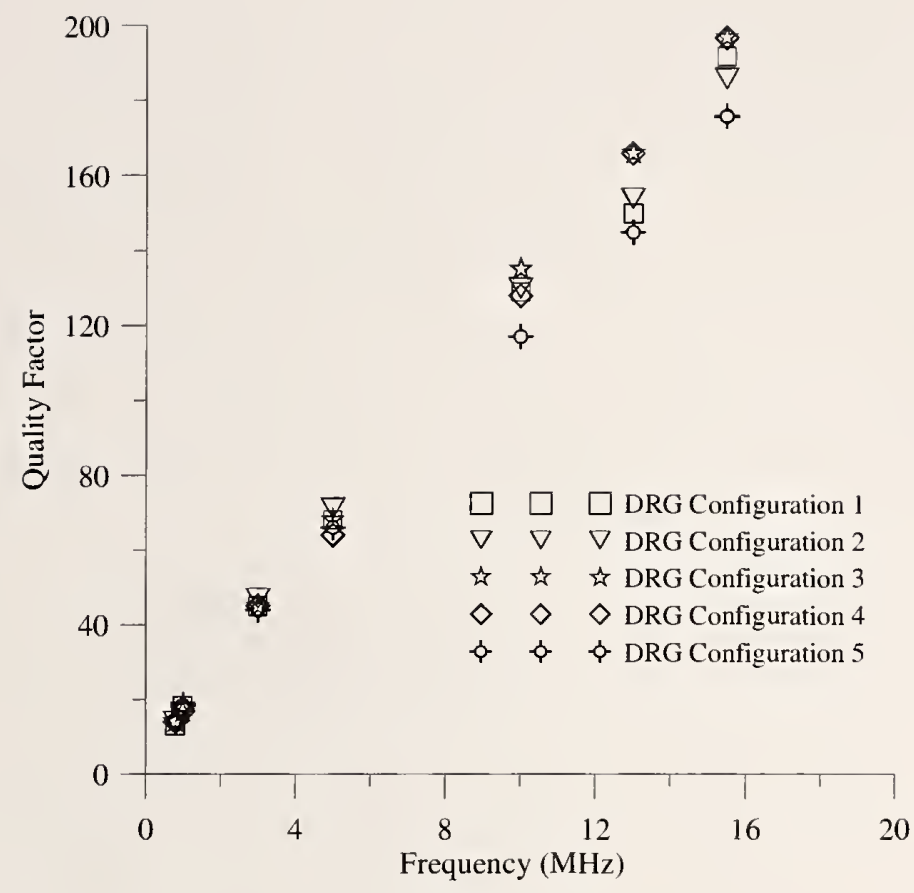

Figure 117. Airframe quality factor (Q) for DRG antennas in all five internal configurations.

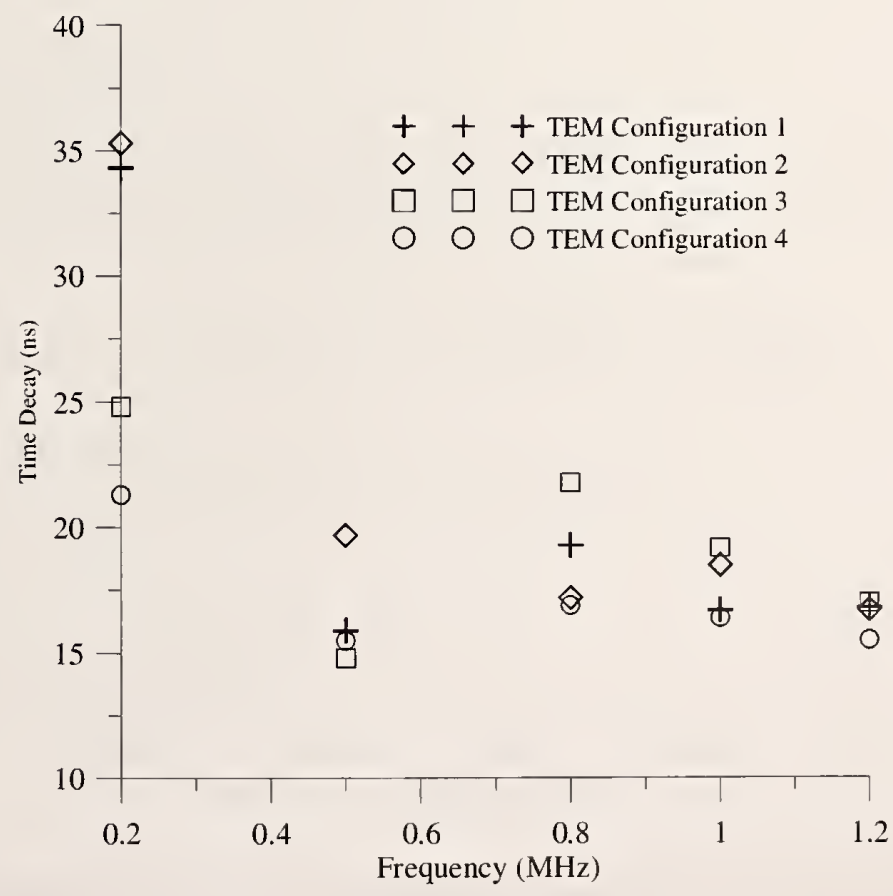

Figure 118. Time decay for TEM horn antennas in all four internal configurations. 


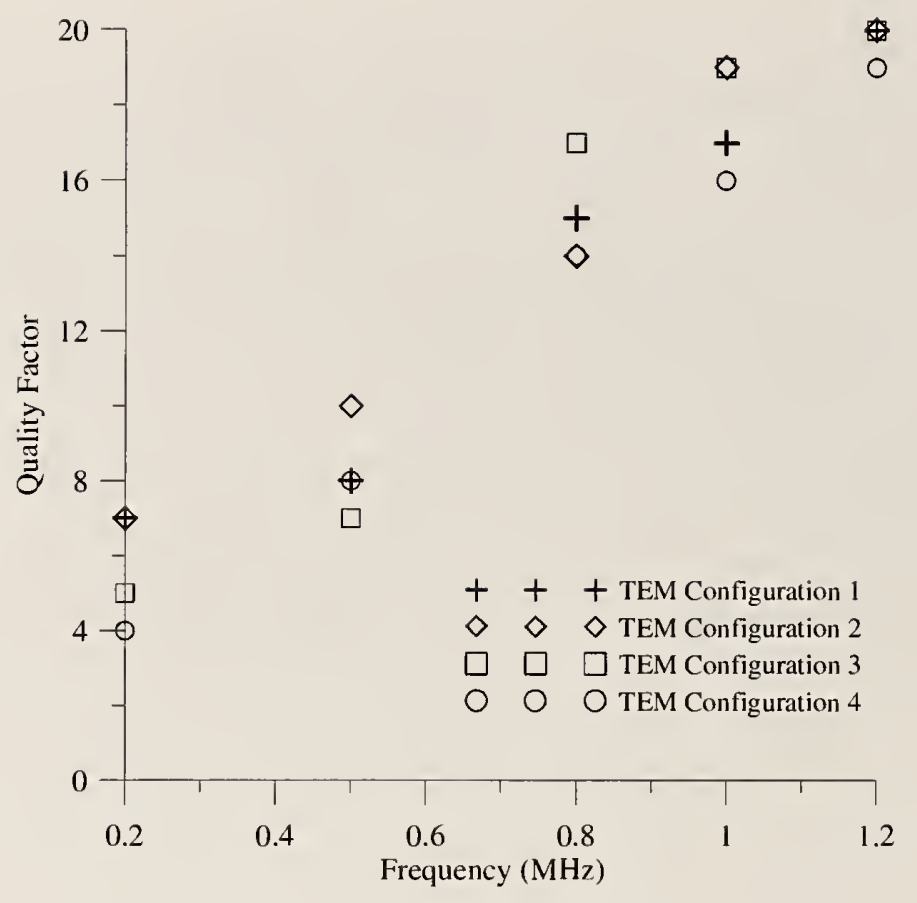

Figure 119. Airframe quality factor (Q) for TEM antennas in all four internal configurations.

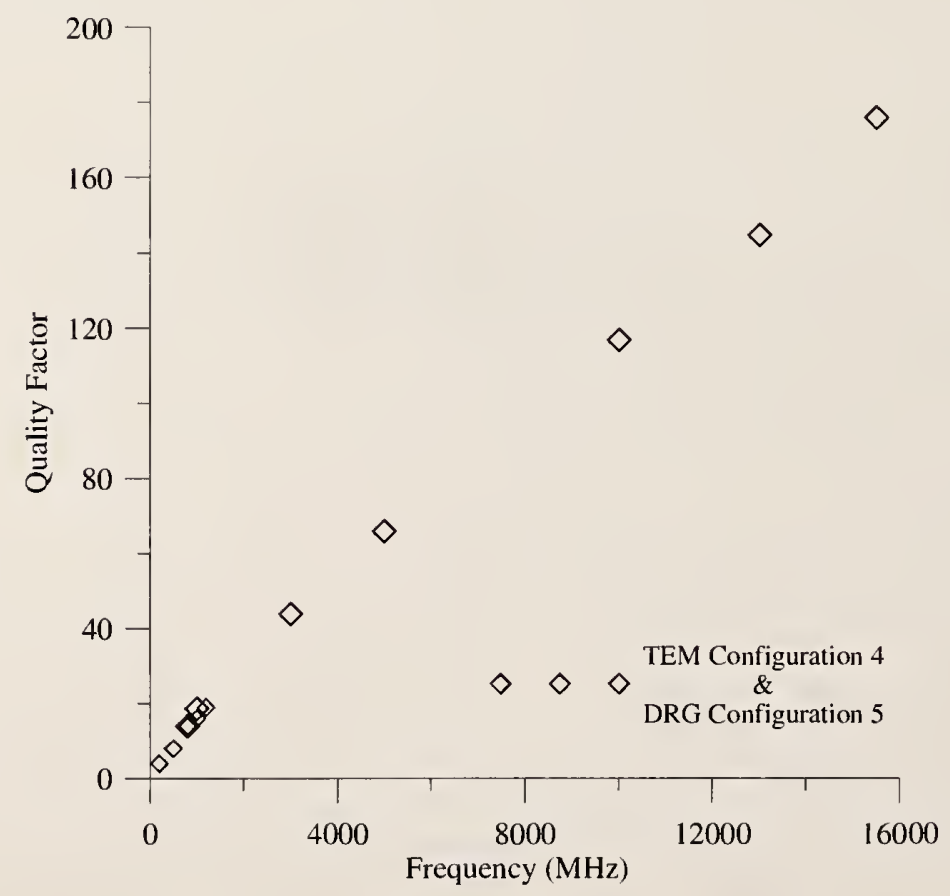

Figure 120. Quality factor for both antennas for DRG Configuration 5 and TEM Configuration 4. 


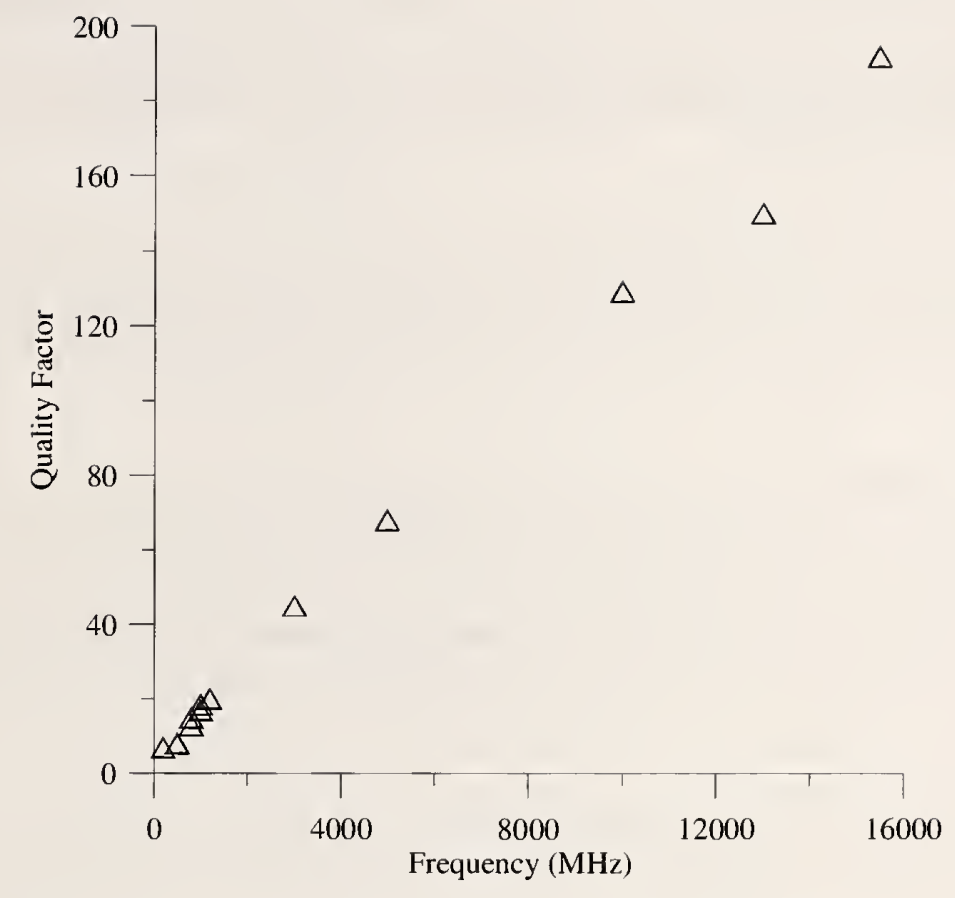

Figure 121. Quality factor for both antennas for Configuration 1.

\section{Uncertainty Analysis}

The sources of uncertainty are based on NISTIR 5019 [7], which provides a natural framework for the identification of source uncertainties encountered in aircraft shielding along with an efficient method of accounting for tracking and combining them.

Six sources of measurement uncertainty are considered in our analysis:

- Measurement Repeatability

- Range Uncertainties/Distance Correction

- $\quad$ Time Gating

- Drift

- Polarization Mismatch

- $\quad$ Signal-to-Noise Ratio

These sources of uncertainties (influence factors) were evaluated as follows:

1. Measurement Repeatability: for a given internal orientation, we took multiple data sets, in which we moved the external antennas to and from the same location. 
2. Range Uncertainties: We used estimates of variations when positioning the antennas for both the reference and airframe measurements

3. Time Gating: We determined time-gating uncertainties by varying the gate width and performing a statistical analysis on the resulting amplitude spectra. See Appendix A for further discussion.

4. Drift: The uncertainty due to instrumentation drift is small. It was computed by using the data obtained from system calibration checks done before and after circumnavigating the aircraft. The uncertainty was determined by looking at the difference and dividing that by the average of the two signals.

5. Polarization Mismatch: Polarization mismatch uncertainties occur when the antennas could be misaligned for a given polarization. We calculated one uncertainty based on a possible misalignment of $5^{\circ}$. The other term comes from the possible leakage from one polarization to the other. The cross-polarization of our TEM horn antennas is approximately $-20 \mathrm{~dB}$, and for the DRG antennas it is approximately $-15 \mathrm{~dB}$. These two factors both contribute to the polarization mismatch uncertainty.

6. Signal-to-Noise Ratio: Signal-t- noise uncertainties were calculated for the Global 5000 aircraft. Appendix B of this report summarizes typical signal and noise characteristics.

The impact of these six influence factors on the measured penetration is summarized in Table 1. This table lists the fractional uncertainties $[7,8]$ resulting from each influence factor. The most significant error is due to uncertainties in the signal-to-noise ratio. Measurement repeatability has an influence that is somewhat less than that of the signal-to-noise ratio, but is nevertheless significant. Time gating is the next largest uncertainty, followed by the range/distance correction uncertainties, extrapolation uncertainties, polarization mismatch, and drift, respectively. Polarization mismatch and drift contribute almost nothing to the overall uncertainty.

The combined standard uncertainty was computed using the procedure given in NIST Technical Note 1297 [9]. The six sources of uncertainties were combined using the root sum-of-squares of the linear fractional uncertainties averaged over a $200 \mathrm{MHz}$ frequency window. The resulting uncertainties (in decibels) for penetration are plotted in Figure 122 for the frequency range from $800 \mathrm{MHz}$ to $1500 \mathrm{MHz}$. The uncertainties were calculated as a function of frequency, and therefore we have listed an approximate average value in the Table 1. We have broken the uncertainties into those below $9 \mathrm{GHz}$ and those above $9 \mathrm{GHz}$, because the noise floor resets itself there due to the internal configuration of both attenuators and mixers in the VNA. We believe that a reasonable uncertainty bound would be at the $95 \%$ confidence level for the data shown in Figure 122, remembering that this current uncertainty analysis is based on a limited set of data. 
Table 1. Typical fractional uncertainties resulting from the six influence factors.

\begin{tabular}{cc|c}
\hline \multirow{2}{*}{ Influence factor } & \multicolumn{2}{c}{ Typical fractional uncertainties } \\
\cline { 2 - 3 } & $<9 \mathrm{GHz}$ & $>9 \mathrm{GHz}$ \\
\hline Signal-to-noise & 1.2 & 1.6 \\
\hline Repeatability & 0.4 & 0.8 \\
\hline Time gating & 0.3 & 0.6 \\
\hline Range/distancecorrection & 0.1 & 0.4 \\
\hline Extrapolation & 0.1 & 0.4 \\
\hline Polarization mismatch & 0.1 & 0.2 \\
\hline Drift & Neg. & Neg. \\
\hline RSS & 1.3 & 2.0 \\
\hline
\end{tabular}

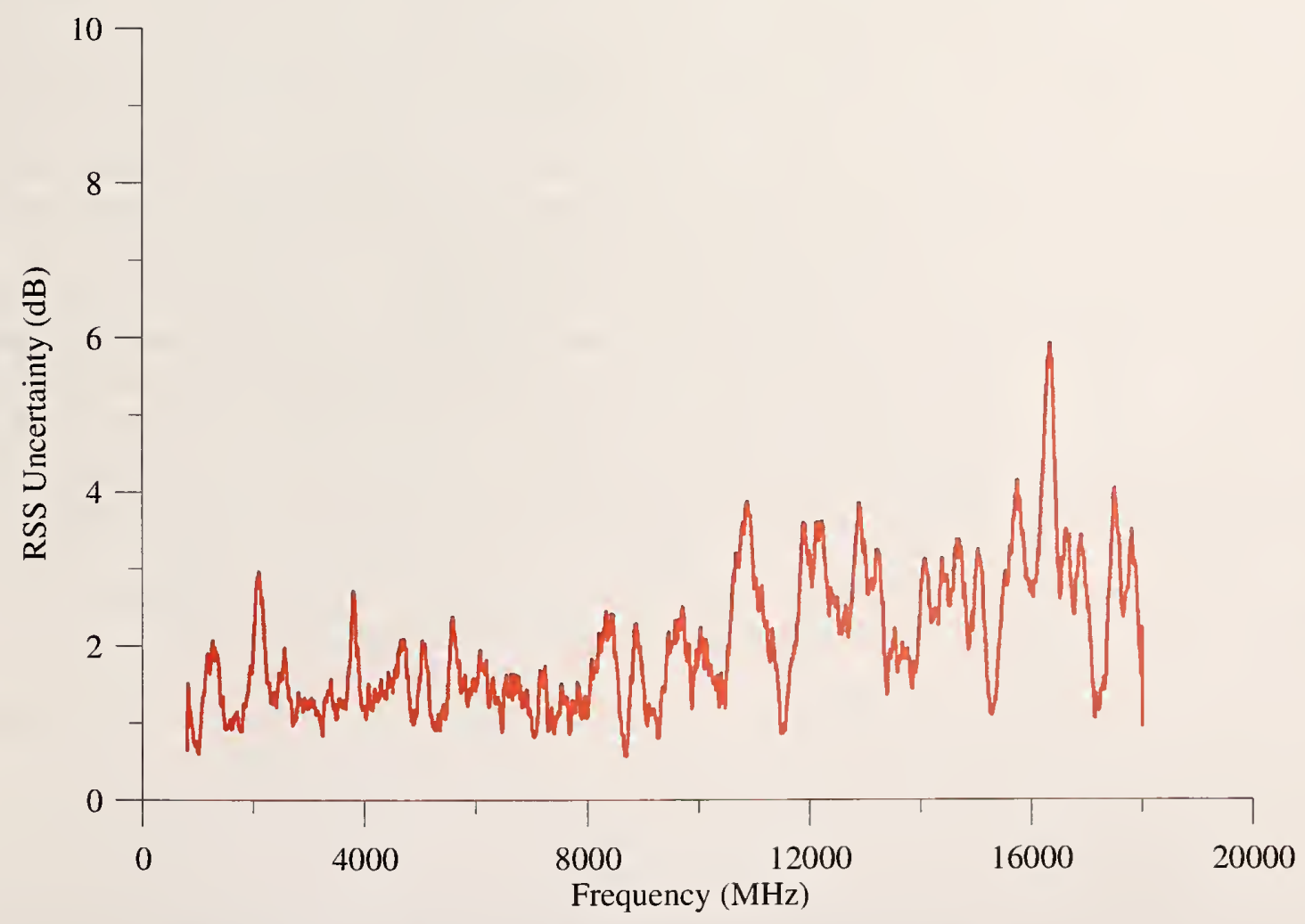

Figure 122. Combined standard uncertainty in penetration. 


\section{Summary and Conclusions}

The data in this report were collected to provide guidelines for HIRF testing on various aircraft. We have reported on the penetration measurements taken of the Beechcraft Premier IA at the Hawker Beechcraft Corporation in Wichita, Kansas. We have discussed extrapolation measurements taken to ensure that the measurements are in the far-field of the transmitting antenna. We have shown results for measurements taken at various azimuth angles around the outside of the aircraft with the receiving antenna in the main passenger cabin, the flight deck, and the rear cargo hatch. We have also performed a series of internal measurements to look at the reverberant field characteristics within the cavity to determine the viable statistics associated with the fields.

The main passenger cabin shows the greatest penetration for all three compartments. We attribute this to the numerous windows. Typical maximum penetration values are $-5 \mathrm{~dB}$ at $900 \mathrm{MHz}$; this same frequency shows maximum penetration around the aircraft. Minimum values of penetration occur at the tail of the aircraft $\left(180^{\circ}\right)$ and are on the order of $-30 \mathrm{~dB}$. Maximum penetration for the flight deck is around $-5 \mathrm{~dB}$ at an angle of $0^{\circ}$ for frequencies around $650 \mathrm{MHz}, 770 \mathrm{MHz}, 1000 \mathrm{MHz}, 1525 \mathrm{MHz}$, and $3100 \mathrm{MHz}$. Minimum penetration occurs at $180^{\circ}$ at a value around $-40 \mathrm{~dB}$. The rear cargo hatch shows maximum penetration values of $-10 \mathrm{~dB}$ from $200^{\circ}$ to $270^{\circ}$, while minimum penetration occurs at $0^{\circ}$ with a value of $-40 \mathrm{~dB}$. With an antenna in the rear cargo hatch, we see low penetration values on the right side of the aircraft from a top-down view. Typically horizontal and vertical polarizations penetrate equally well into the aircraft; however, at the lower frequencies the horizontal polarization has higher penetration values and occurs toward the tail of the aircraft. We found that symmetry is maintained around the aircraft. HIRF penetration into the flight deck shows approximate values of $-5 \mathrm{~dB}$ to $-10 \mathrm{~dB}$ at lower frequencies and $-15 \mathrm{~dB}$ at higher frequencies for a radial distance of $15 \mathrm{~m}$ from the front landing gear at angles of $270^{\circ}, 315^{\circ}, 0^{\circ}, 45^{\circ}$, and $90^{\circ}$. The internal coupling measurements showed that traditional reverberation statistics would not be valid in this cavity, but good agreement was shown between the two antenna types for quality factor values between $1 \mathrm{GHz}$ and $2 \mathrm{GHz}$. Finally, we found that major coupling apertures would not be missed if measurements were taken every $10^{\circ}$ as opposed to every $5^{\circ}$. Figure 123 shows results for the DRG horn antenna in both vertical and horizontal polarizations for various frequencies in each compartment. Figure 124 shows results for the TEM horn antenna in both vertical and horizontal polarizations for various frequencies in each compartment. Red circles are for the main cabin, blue triangles are for the flight deck, and green squares are for the rear cargo hatch. This shows us that rear cargo hatch penetration is typically lower than that for either the main passenger cabin or the flight deck at each of these frequencies. We also see that the penetration values are fairly constant around the aircraft, which we believe is due the use of carbon-fiber composite material. And we see that penetration typically decreases as frequency increases. 


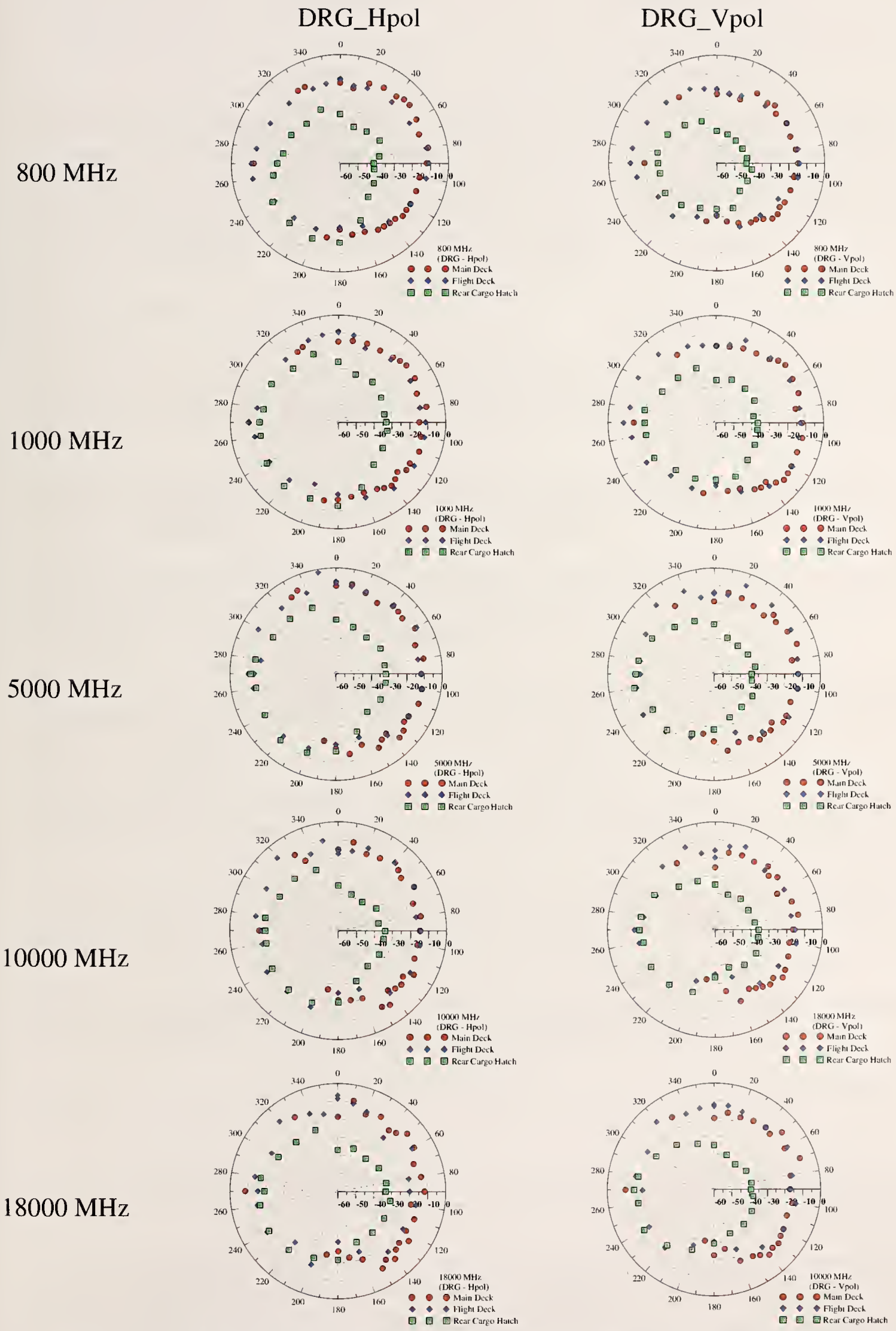

Figure 123. Graphs for DRG horn antenna in both vertical and horizontal polarizations for various frequencies in each compartment. Red circles $(O)$ are for the main cabin, blue diamonds $(\diamond)$ are for the flight deck, and green squares $(\square)$ are for the rear cargo hatch. 

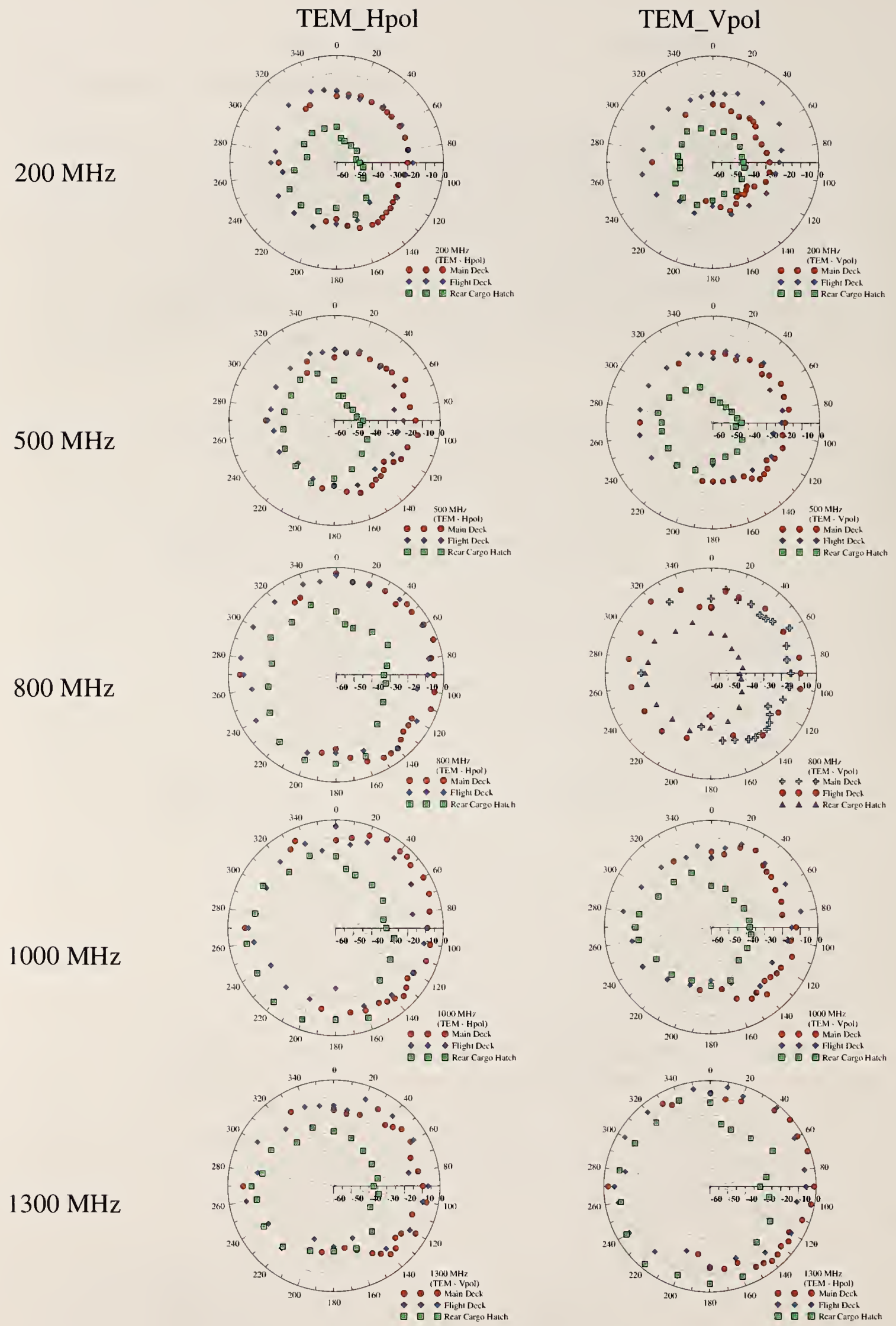

Figure 124. Graphs for TEM horn antenna in both vertical and horizontal polarizations for various frequencies in each compartment. Red circles $(\Theta)$ are for the main cabin, blue diamonds $(\diamond)$ are for the flight deck, and green squares $(\square)$ are for the rear cargo hatch. 
We thank Howard Jordan from the Hawker Beechcraft Corporation in Wichita, Kansas for allowing us to measure one of their carbon composite aircraft and we thank David Walen from the FAA office in Renton, Washington for his sponsorship and financial support for this work. This was an important measurement effort both to aid in understanding and to help define standard measurement procedures for HIRF testing.

\section{References}

[1] C.A. Grosvenor, D. Novotny, D. Camell, G. Koepke, R.T. Johnk, Electromagnetic airframe penetration measurements for the FAA Bombardier Global 5000, Natl. Inst. Stand. Technol. Tech. Note 1547 (May 2008).

[2] SAE Aerospace Group, Guide to certification of aircraft in a high intensity radiated field (HIRF) environment, SAE ARP5583 (January 2003).

[3] C.A. Grosvenor, R.T. Johnk, D.R. Novotny, N. Canales, C.M. Weil, J. Veneman, A twophase airframe shielding performance study using ultrawideband measurement systems, Natl. Inst. Stand. Technol. NISTIR 6622 (January 2003).

[4] P.F. Wilson, D.A. Hill, C.L. Holloway, On determining the Maximum Emissions From Electrically Large Sources, 2002 IEEE Trans. Electromagnetic Compatibility, 44(1): 79-86 (February 2002).

[5] R.T. Johnk, B. Archambeault, D. Novotny, Using joint time-frequency analysis to enhance time-domain numerical emc simulations, 2003 Annual Report Interference Technology (2003).

[6] J.M. Ladbury, T. H. Lehman, G.H. Koepke, Coupling to devices in electrically large cavities or why classical emc evaluation techniques are becoming obsolete, 2002 Intl. Symp. on Electromagnetic Compatibility, 2: 648-655 (August 2002).

[7] R.C. Wittmann, M.H. Francis, L.A. Muth, R.L. Lewis, Proposed uncertainty analysis for RCS Measurements, Natl. Inst. Stand. Technol. NISTIR 5019 (January 1994).

[8] J.R. Taylor, An introduction to error analysis, University Science Books, Sausolito, CA. (1997).

[9] B.N. Taylor, C.E. Kuyatt, Guidelines for evaluating and expressing the uncertainty of NIST measurement results, Natl. Inst. Stand. Technol. Tech. Note 1297 (September 1994).

[10] E.B. Larsen, R.L. Ehret, D.G. Camell, and G.H. Koepke, Calibration of antenna factor at a ground screen field site using an automatic network analyzer, 1989 Intl. Symp. on Electromagnetic Compatibility, 1: 19-24 (August 1989). 
[11] http://www.chm.bris.ac.uk/webprojects2002/mjames/chemistry.html. Accessed March 10, 2008.

[12] http://www.geocities.com/vpkelly.geo/blog.html?t=p\&pg=1\&cnt=5. Accessed March 10, 2008.

[13] http://en.wikipedia.org/wiki/Graphite-reinforced plastic. Accessed March 10, 2008.

[14] F. Sagnard, A. Berthault, B. Véron, The microwave measurement of the conductivity of a small fiber with an open cavity, IEEE Trans. Instrum. Meas., 49(5): 942-948 (October 2000).

[15] C.A. Johnk, Engineering electromagnetic fields and waves, $2^{\text {nd }}$ ed., New York : J. Wiley and Sons, pg. 168 (1988).

[16] M.J. Akhtar, L.E. Feher, M. Thumm, A waveguide-based two-step approach for measuring complex permittivity yensor of uniaxial composite materials, IEEE Trans. Microwave Theory Tech., 54(5): 2011-2022 (May 2006).

[17] S.P. Rea, D. Wylie, D. Linton, E. Orr, J. McConnell, EMI shielding of woven carbon Fibre composites, 2004 High Frequency Post-Graduate Student Colloquium, pp. 205-210 (September 2004).

[18] J. Ding, S. Rea, D. Linton, E. Orr, J. McConnell, Mixture properties of carbon fibre composite materials for electronics shielding in systems packaging, 2006 Electronics System Integration Technology Conf., 1: 19-25 (September 2006).

[19] C.L. Holloway, M.S. Sarto, M. Johansson, Analyzing carbon-fiber composite materials with equivalent-layer models, IEEE Trans. Electromag. Compat., 47(4): 833-844 (November 2005).

[20] M. Lin, C.H. Chen, Plane-wave shielding characteristics of anisotropic laminated composites, IEEE Trans. Electromag. Compat., 35(1): 21-27 (February 1993).

[21] H. Chu, C.H. Chen, Shielding and reflection properties of periodic fiber-matrix composite structures, IEEE Trans. Electromag. Compat., 38(1):1-6 (February 1996). 


\section{Appendix A. Time-Domain Waveform Analysis}

The time-domain waveforms can tell us a lot about the penetration into the airframe. Figure 125 shows the time-domain waveform for a DRG antenna transmitting in the horizontal polarization at an angle of $30^{\circ}$ for a receiving antenna located in the main passenger cabin. The figure shows the linear time-domain response (red trace) and the log-magnitude response (black trace) of the signal versus time. Using both representations it is easy to define the direct component (green box), the first-time gate position (blue box), the second time-gate position (magenta box), and why we either include or exclude certain portions of the signal in our analysis. The first piece of information we can get is the time-of-flight between the transmitting and receiving antenna. This is shown by the onset of the direct component. This can give us insight about the distance between the two antennas. For this measurement we tried to position our transmitting antennas on a $23.5 \mathrm{~m}$ circle, with reference to the geometric center, which translates to $75 \mathrm{ft}$. Taking a close look at the onset of our direct component, as shown in Figure 126, shows us that the time of flight corresponds to $73.3 \mathrm{~ns}$, and using the conversion that there are $0.983 \mathrm{ft}$ per ns of time of flight, we conclude that our actual distance was probably $22.0 \mathrm{~m}(72.1 \mathrm{ft})$. The direct component is the antenna-to-antenna response as the signal enters the aircraft. The response is short in duration, and in a log-magnitude format has a rapid decay time. The portion of the signal after the initial response is due to the reverberant fields that develop from the energy that bounces around in the cavity due to this initial electromagnetic energy. These have a different decay characteristic. At $250 \mathrm{~ns}$ we apply a stop time gate where we zero the rest of the waveform; this is shown by the blue box. Notice that we try to apply a stop time gate at a point close to the noise floor of the signal response, which helps decrease the oscillation in the frequency domain due to the application of a windowing function. We then want to look at the tiny bit of energy shown building up and decaying between $250 \mathrm{~ns}$ and $550 \mathrm{~ns}$ to see whether this energy affects

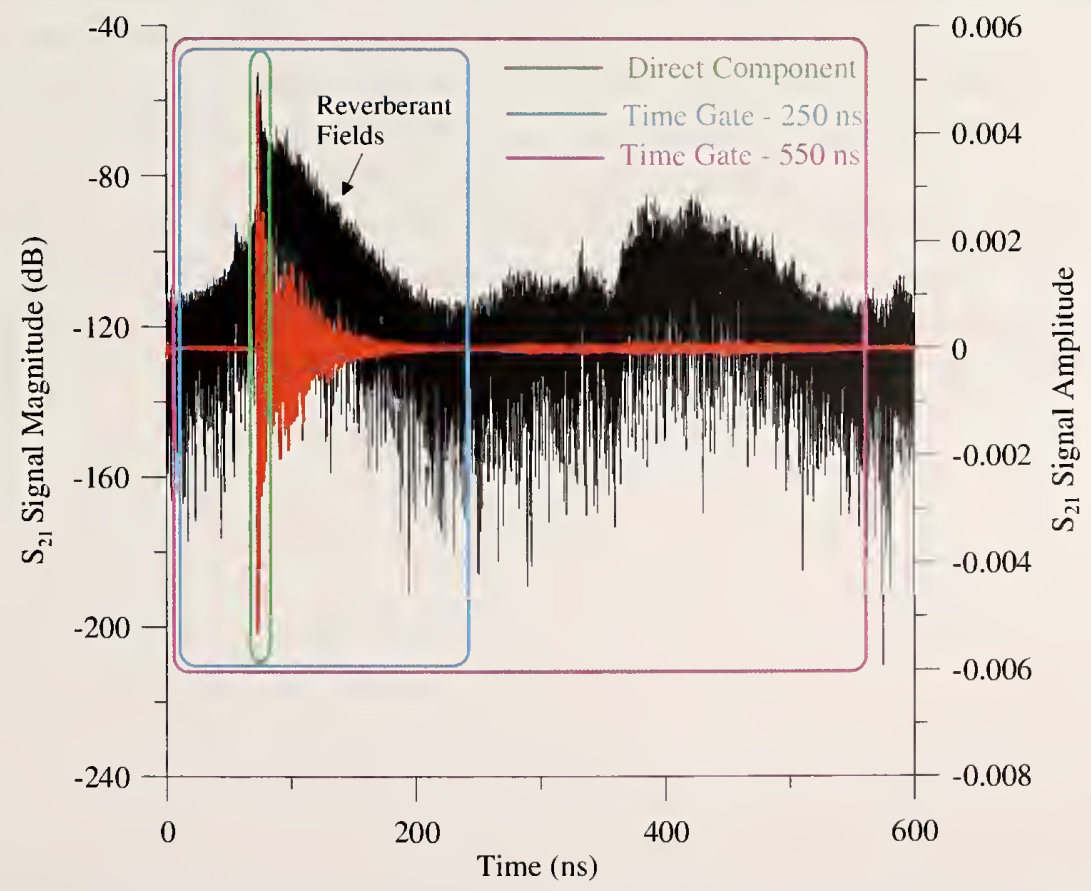

Figure 125. Time-domain waveforms for a DRG antenna transmitting at angular position $30^{\mathrm{c}}$. Receiving antenna is in the main passenger cabin. 


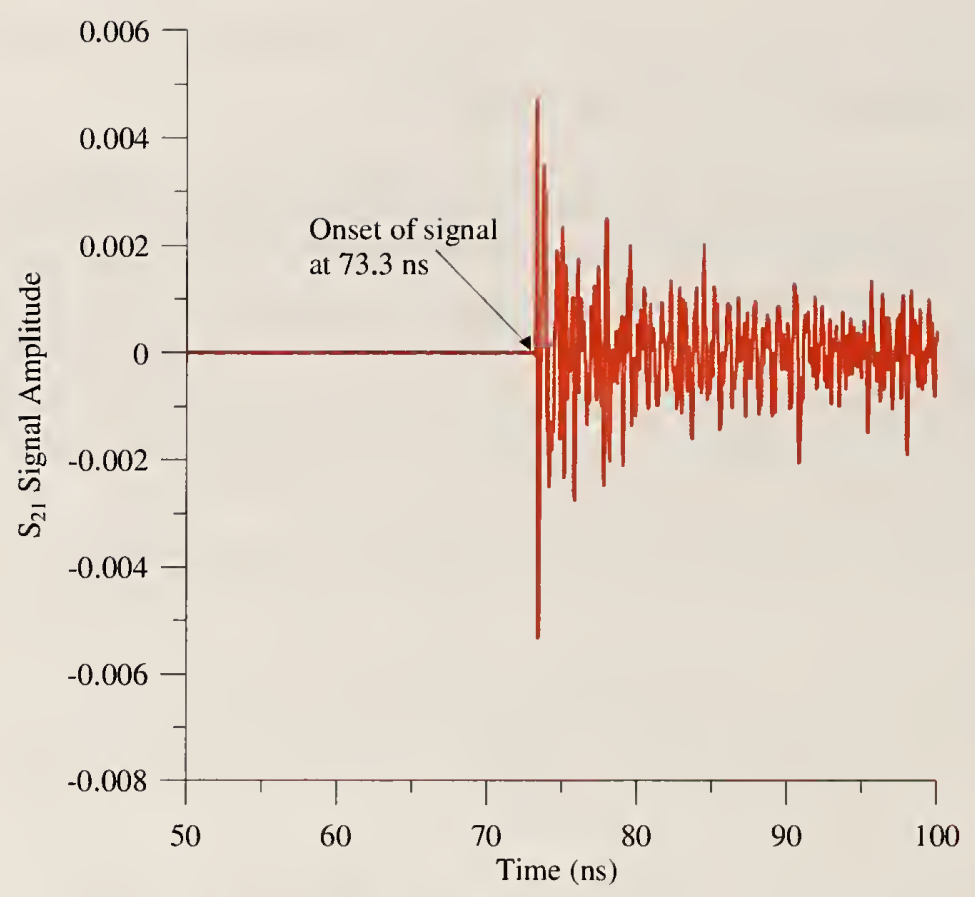

Figure 126. Timewaveform for a DRG antenna transmitting at angular position $30^{\mathrm{c}}$ and for the receiving antenna in the main passenger cabin. Onset of signal occurs at $73.3 \mathrm{~ns}$.

our application of the time gate. Since this energy translates to an event occurring at approximately $250 \mathrm{ft}$, we believe this event occurs outside the aircraft; but if it is a small buildup of energy somewhere inside the aircraft, then we want to make sure we include it if it is important in the measurement of this aircraft. Therefore, in the application of our second gate we include this amount of energy and look at its impact in the frequency domain. This is shown in Figure 127. The black trace is the penetration without time gating the waveform; in other words, it is the full time-domain record. The red trace is the penetration for time gating from 10 ns to $250 \mathrm{~ns}$, so that anything that happens outside this period is removed from the record. The blue trace shows the waveform that includes time-domain information from $10 \mathrm{~ns}$ to $550 \mathrm{~ns}$. Since the blue trace obscures the black and red traces, we can say that the extra 300 ns added to the blue trace contains little more information than what was already obtained by the red trace, so we would be safe to stop the time gate at $250 \mathrm{~ns}$ and be sure that we are not missing important information from the time-domain signal. 


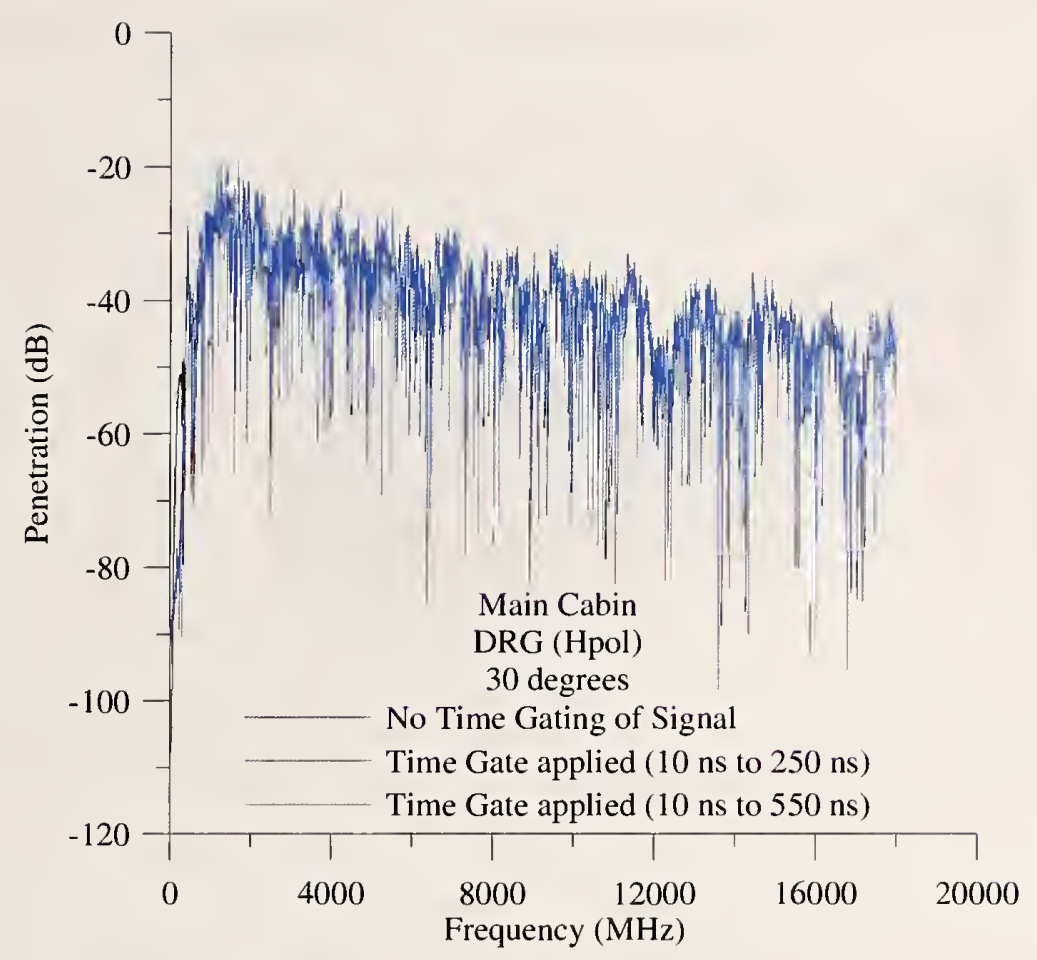

Figure 127. Frequency-domain waveforms for a DRG antenna transmitting at angular position of $30^{\circ}$. Receiving antenna is located in the main passenger cabin.

Let us examine the signal at another position. This signal was obtained with the transmitting antenna located at an angle of $220^{\circ}$ and the receiving antenna located in the rear cargo hatch. The time-domain signal is shown in Figure 128. Notice that for this compartment there is no direct signal path, and the only signal that enters this compartment is of low amplitude and immediately decays into reverberant fields. Also no pronounced signal amplitude is observed after $600 \mathrm{~ns}$, where we reach our noise floor, and so the only thing we add to our signal at this point is noise. This is why we choose to time gate the signal, so that most of the signal is analyzed versus signal plus noise. The frequency-domain signal is shown in Figure 129. The black curve shows the entire waveform without time-domain gating, and the red curve shows the signal once a time-gate is applied from $10 \mathrm{~ns}$ to $600 \mathrm{~ns}$. This shows that because the signal has decayed to the noise floor by $600 \mathrm{~ns}$, the noise increases the amplitude of the signal only a little. 


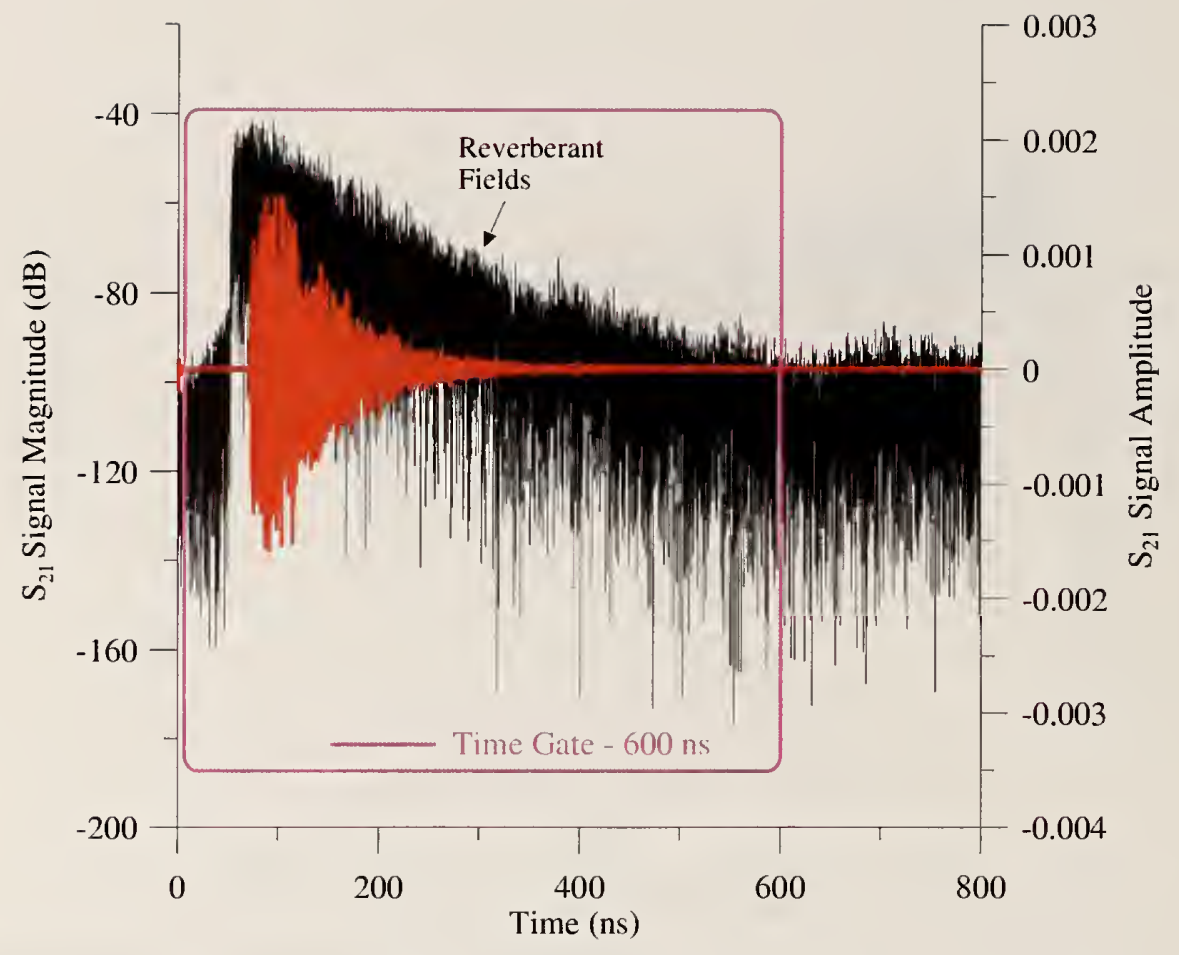

Figure 128. Time-domain waveforms for a DRG antenna transmitting at an angular position of $220^{c}$. Receiving antenna located in the rear cargo hatch.

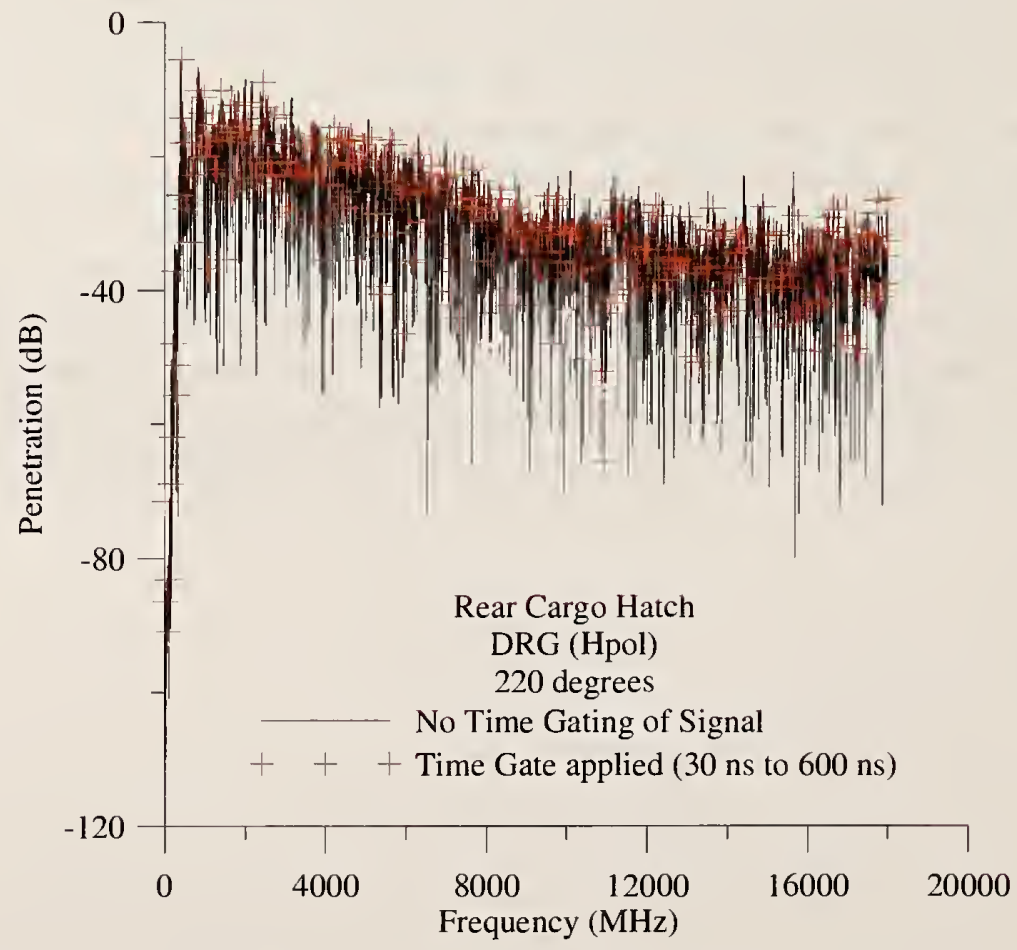

Figure 129. Frequency-domain waveforms for a DRG antenna transmitting at an angular position of $220^{\circ}$. Receiving antenna located in the rear cargo hatch. 


\section{Appendix B. Signal and Noise Characteristics}

Typical signal and noise characteristics are shown in Figures 130 to 132 for the three compartments: (1) main passenger cabin, (2) flight deck, and (3) rear cargo hatch, at three different angular positions: (1) $90^{\circ}$, (2) $20^{\circ}$, and (3) $180^{\circ}$, respectively. We have plotted only the DRG horn antenna in the horizontal polarization for this section. Noise is a function of position due to the location of ambient RF sources that the aircraft may shield from the internal antennas as we move around the aircraft. Noise floors tend to be around $-60 \mathrm{~dB}$ to $-80 \mathrm{~dB}$ at the lower frequencies and climb to around $-50 \mathrm{~dB}$ at the higher frequencies. The rise in noise floor at around $9 \mathrm{GHz}$ is due to the attenuator configuration of the VNA for upper frequencies. The signal-to-noise ratios for the main passenger cabin vary from $30 \mathrm{~dB}$ to $60 \mathrm{~dB}$. In the flight deck, the signal-to-noise ratio varies from $30 \mathrm{~dB}$ to $55 \mathrm{~dB}$, and in the rear cargo hatch, the signal-tonoise ratio varies from $15 \mathrm{~dB}$ to $55 \mathrm{~dB}$.

The signal-to-noise characteristics for the TEM horn antennas are a little different, due to the FM frequency bands. For the frequency band from $100 \mathrm{MHz}$ to $1500 \mathrm{MHz}$, typical signal-tonoise ratios vary from $20 \mathrm{~dB}$ at the lower frequencies to $60 \mathrm{~dB}$ at the higher frequencies.

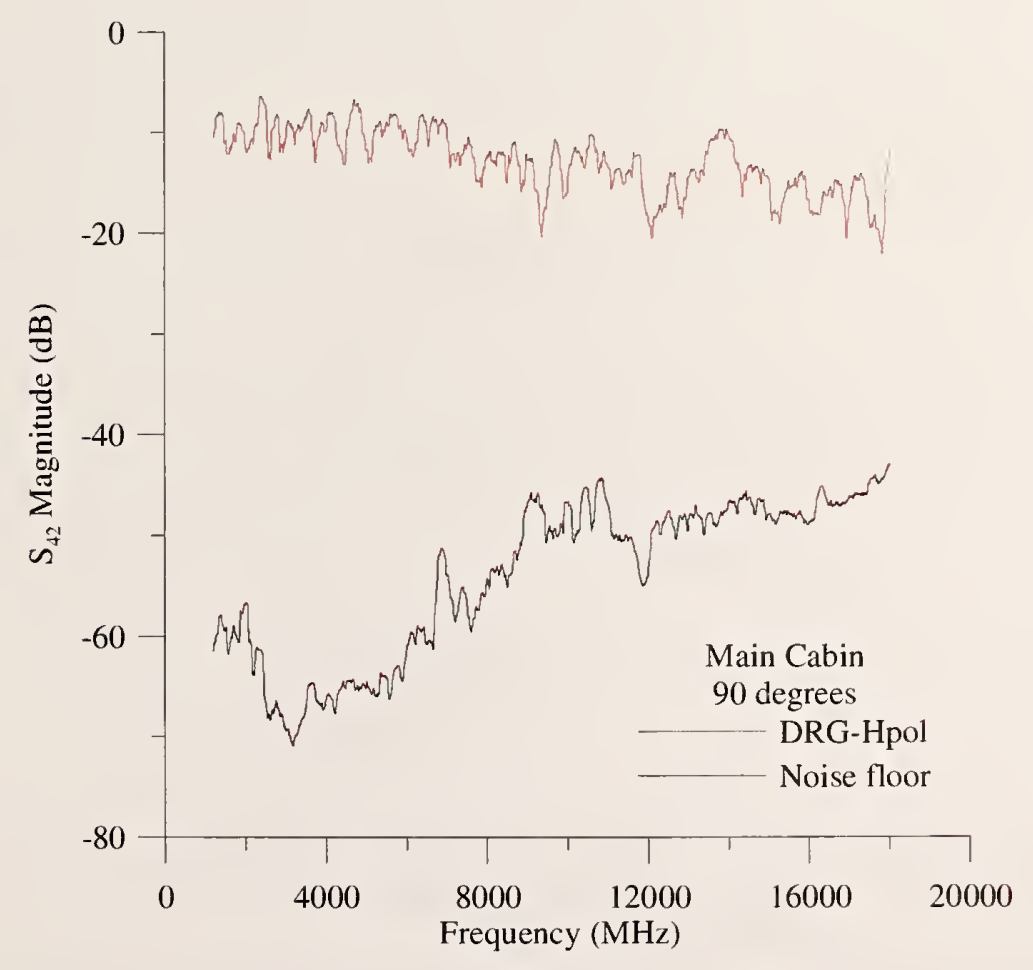

Figure 130. Typical signal and noise characteristics for the main passenger cabin. This particular noise measurement was taken at an angle of $90^{\circ}$. 


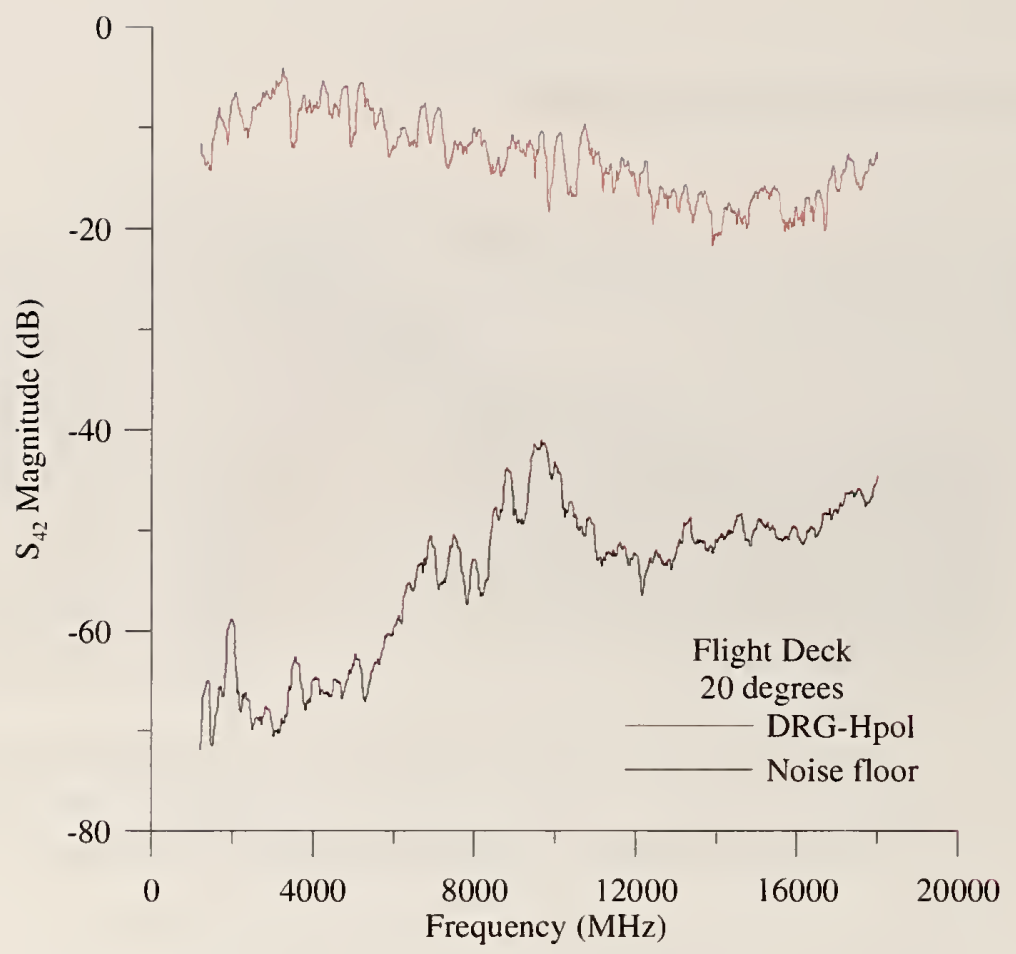

Figure 131. Typical signal and noise characteristics for the flight deck. This particular noise measurement was taken at an angle of $20^{\circ}$.

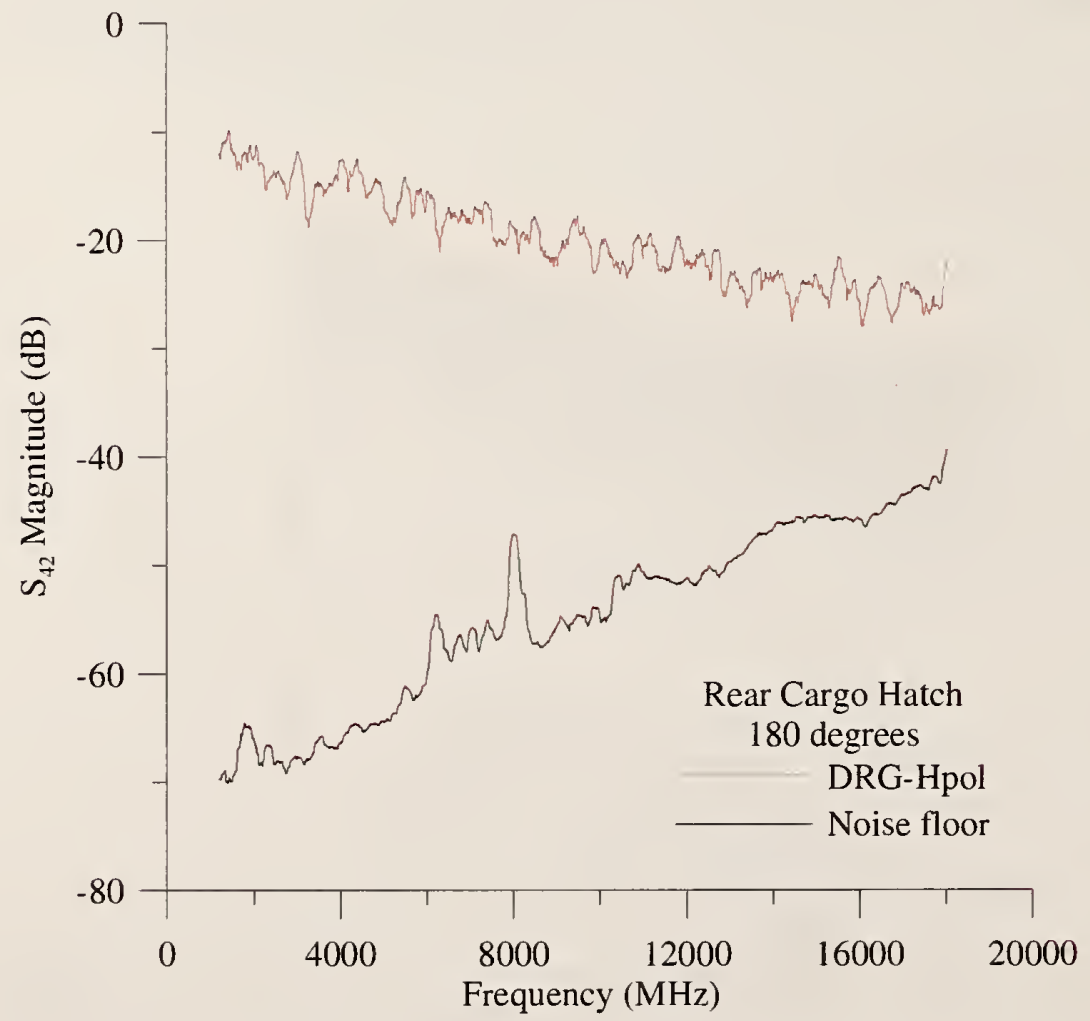

Figure 132. Typical signal and noise characteristics for the cargo closet. This particular noise measurement was taken at an angle of $180^{\circ}$. 


\section{Appendix C: TEM and DRG Horn Boresight Gain Characteristics}

The DRG and TEM horn antennas used in this effort were calibrated before we deployed the system. This technique can be used to derive antenna gain with specified resistive loads (typically $50 \Omega$ ) at the antenna terminals. An accurate method for doing this is the three-antenna method. As the name implies, three transmission measurements are made with three combinations of the three antennas. This procedure is described in detail in reference [10]. This process yields three equations that can be readily solved for either gain or antenna factor. The gain characteristics of our DRG horn antennas are shown in Figure 133, the gain characteristics of our $36 \mathrm{~cm}$ TEM horns are shown in Figure 134, and the gain characteristics of our $1.2 \mathrm{~m}$ TEM horns are shown in Figure 135. The gains displayed in these figures include the antenna input mismatch, which accounts for the reduction of gain at the lower frequencies. The DRG horn antenna gain, plotted in Figure 133, shows that the low-frequency cutoff of these antennas occurs around $750 \mathrm{MHz}$. As the frequency is reduced, the input mismatch increases, which accounts for a rapid decrease in antenna gain. Mismatch and gain are not cause and effect but are instead due to a combination of aperture beam forming, and a better input match accounts for the increasing gain at the higher frequencies. A maximum gain of approximately $15 \mathrm{~dB}$ occurs at $16000 \mathrm{MHz}$. The $36 \mathrm{~cm}$ antenna results, plotted in Figure 134, shows a maximum gain of approximately $7 \mathrm{~dB}$ realized at $4000 \mathrm{MHz}$. Results for a NIST $1.2 \mathrm{~m}$ horn antenna are plotted in Figure 135. This horn is longer and has a resistive taper, which improves the low-frequency performance. The maximum frequency of this antenna is $1500 \mathrm{MHz}$, due to balun performance limitations, and a maximum gain of $7 \mathrm{~dB}$ occurs at this frequency as well.

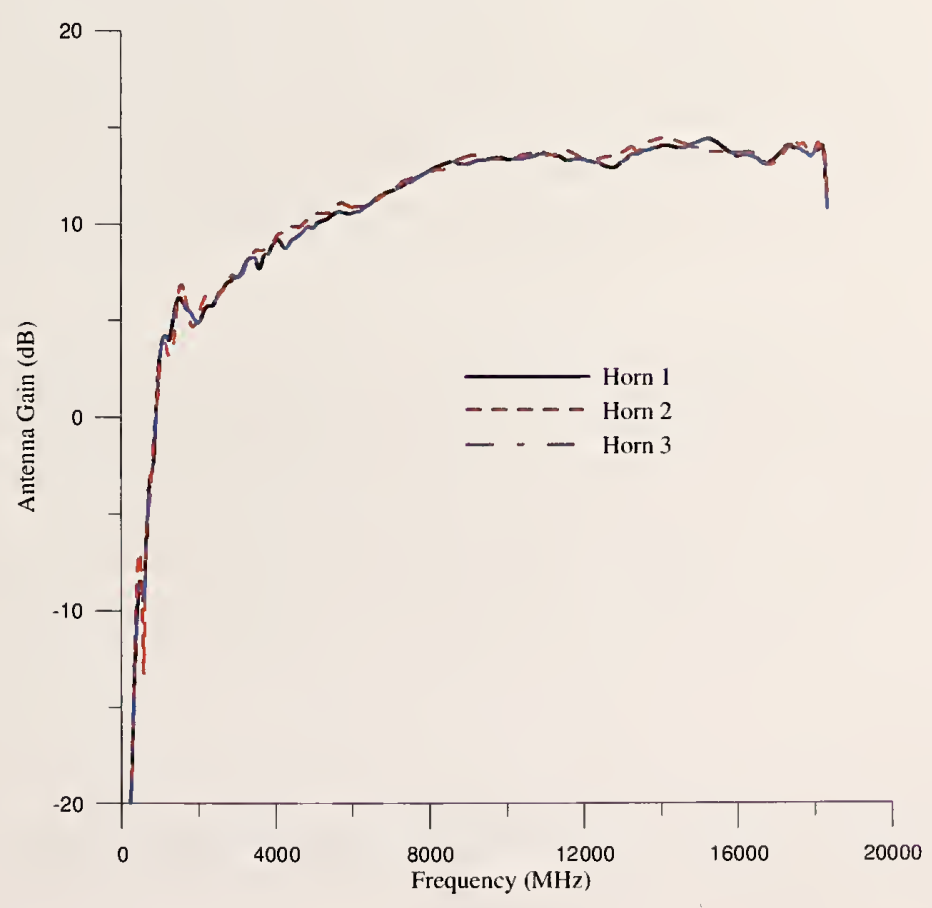

Figure 133. Measured boresight antenna gain for a 3117 DRG horn antenna. The gains plotted here include the input mismatch effects, which account for the rapid decrease below $750 \mathrm{MHz}$. 


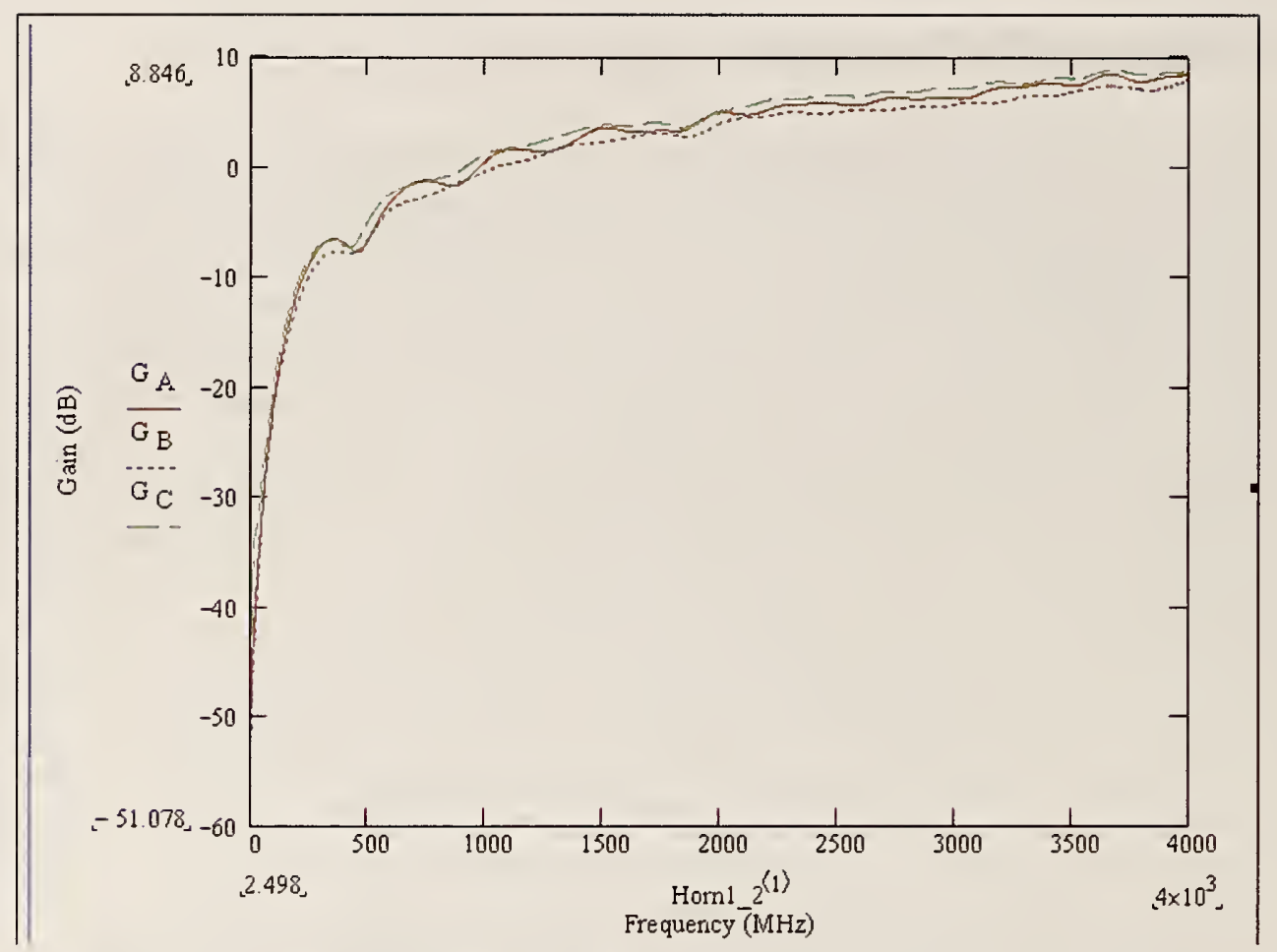

Figure 134. Measured boresight antenna gain for three identical NIST $36 \mathrm{~cm}$ TEM horn antennas. The gains plotted here include the input mismatch effects, which account for the rapid decrease below $100 \mathrm{MHz}$.

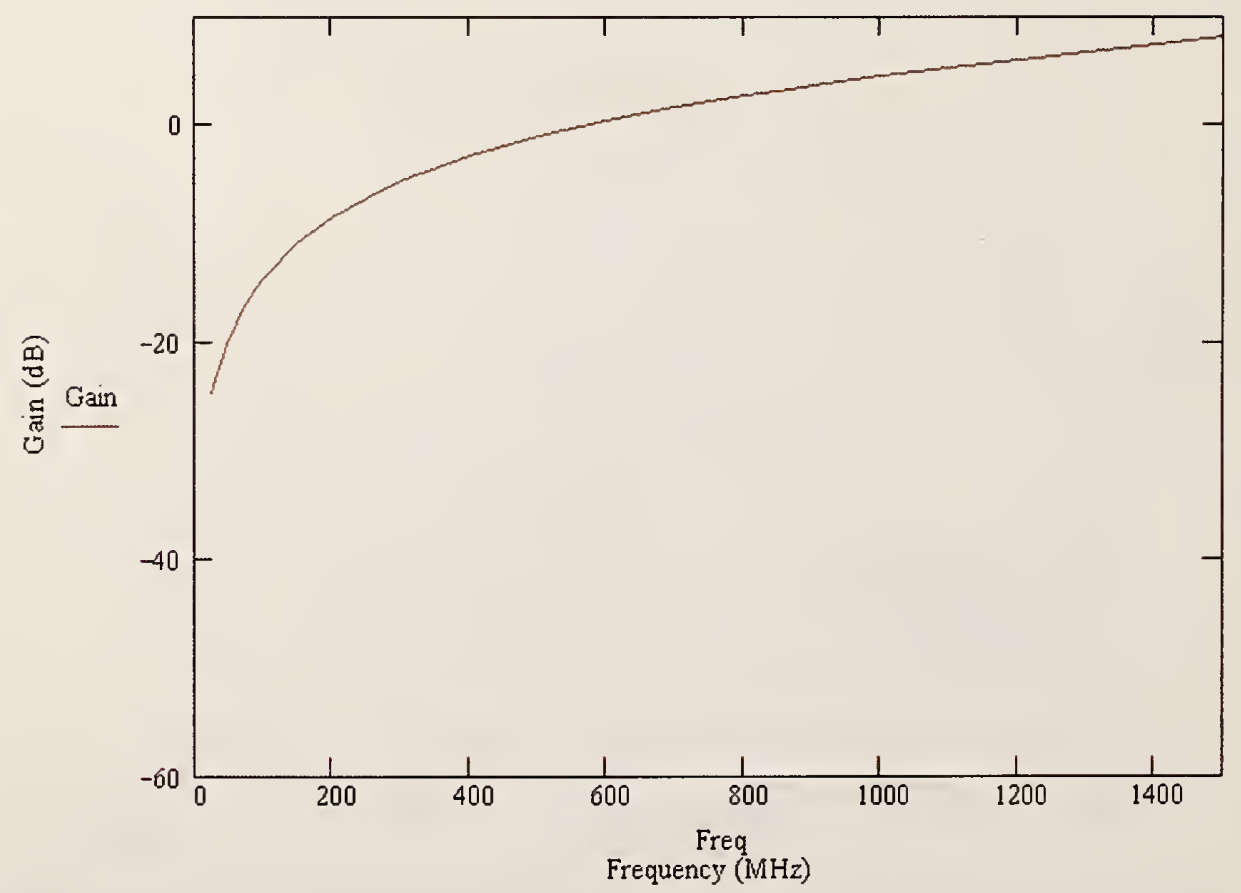

Figure 135. Measured boresight antenna gain for a NIST $1.2 \mathrm{~m}$ TEM horn antenna. The gains plotted here include the input mismatch effects, which account for the rapid decrease below 50 $\mathrm{MHz}$. 


\section{Appendix D. Carbon Fiber Composites}

The use of carbon fiber composites is increasing, due to their high tensile strength, resistance to corrosion, and light weight. Carbon fiber parts are used in automobile racing and limitedproduction automobiles, as well as in fairings, motorcycle mudguards, boats, and aircraft. Carbon fiber is being investigated for use in structural engineering in applications such as bridges, concrete structures, and beams, because of its strength and resistance to corrosion. Carbon fiber is also making its way into many other areas of manufacturing. There is a wide variation in carbon-fiber composite materials, so this appendix is provided only to add understanding of how carbon composite materials behave in electromagnetic fields and to understand how they are fabricated. This information is not specific to the tested aircraft because different manufacturers use different materials and processes.

Carbon fiber has many desirable properties. It is resistant to corrosion and fire; it has high levels of stress tolerance; and it is chemically inert and unreactive. Carbon fiber has a higher tensile strength than steel [11]. Carbon fiber has a tensile strength of 3.50, higher that that of steel, whose tensile strength is only 1.30. It is lighter than steel by a factor of 4.5 .

At very high temperatures, around $2500{ }^{\circ} \mathrm{C}$, carbon fiber can be converted to graphite directly, and therefore has the same chemical structure. The carbon atoms that constitute both carbon fiber and graphite arrange themselves into honeycomb-like sheets lying on top of each other. These sheets are, however, less ordered than graphite sheets, and have weak bonds between layers, and the sheets fold within an individual fiber. Both structures are brittle; however, carbon fiber is less stable than graphite. Each fiber strand is typically made from thousands of carbon filaments and these strands are woven together to form the basis of the part. Since only three carbon atoms are used in interatomic bonding, the fourth atom contributes a free electron to the matrix, allowing it to act as a conductor even though it is nonmetallic. A spool of carbon strands is shown in Figure 136 [12].

Carbon fiber composites are materials made from many strands of carbon embedded in some type of resin. To produce graphite epoxy parts [13], sheets of woven carbon fiber are layered over a mold and oriented to provide optimal strength for the part. Once air is evacuated from the mold, an epoxy resin is applied and, depending on the part, may be either heated or air-cured. More information on the process can be found in the references [10-13]. Epoxy resin is used to hold the shape of the part and to give it rigidity in the final product. 


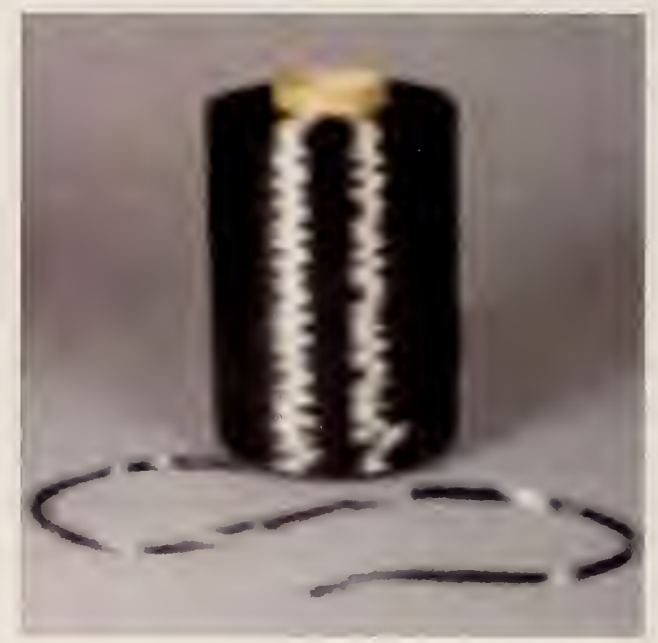

Figure 136. Spool of carbon fiber strand [12].

Now that we understand the structure of carbon fiber composites, we can begin to explore their electrical properties and how electromagnetic fields will interact with them. This section presents measurements of the dielectric constant, the loss tangent, and the conductivity, then the last section describes their shielding characteristics. By having a good understanding of the electrical properties, we can begin to understand how the shielding effectiveness (SE) might be influenced by adopting a metallic, rather than a nonmetallic, structure.

\section{D.1 Dielectric Properties}

We begin with a microwave measurement of the conductivity of some carbon-fiber inclusions in various dielectric matrices [14]. These measurements were performed in a Fabry-Perot resonator at a frequency of $10 \mathrm{GHz}$. The inclusions were made from Torayca T300 with a stated conductivity of $5.5 \cdot 10^{4} \mathrm{~S} / \mathrm{m}$. The average length of the inclusions was $3.25 \mathrm{~mm}$ with a radius of $4 \mu \mathrm{m}$. The measured conductivities have an average value of $6.1 \cdot 10^{4} \mathrm{~S} / \mathrm{m} \pm 15 \%$. The conductivity of aluminum is around $3.5 \cdot 10^{7} \mathrm{~S} / \mathrm{m}[15]$. So the conductivity measured in this experiment is about 1000 times less than that of aluminum.

A material's conductivity is directly associated with the imaginary part of the dielectric constant [16]. In this study, a waveguide transmission method was used to determine the dielectric constant and effective conductivity (loss tangent) for the electric field both parallel and perpendicular to the fiber orientation. Several well known materials were measured, along with four different types of carbon fiber-reinforced polymers. The dielectric properties of the well known materials are used as a check against the measured properties of the carbon-fiber composite materials. For the carbon-fiber composites, for the field in the parallel orientation, the dielectric constant showed values between 1500 and 3500, and in the perpendicular direction, the dielectric constant showed values between 34 and 55. There is thus a high degree of anisotropy in these types of materials, and so the orientation of the fibers with respect to the electric field 
orientation, is critical. The conductivity is also strongly affected by the orientation of the material with respect to the electric field. The conductivity measured here is an effective conductivity, because the conductivity of the resin matrix cannot be separated from that of the carbon fiber. The data show that, for an electric field component parallel to the fiber orientation, the effective conductivity has values from $30 \mathrm{~S} / \mathrm{m}$ to $110 \mathrm{~S} / \mathrm{m}$; and in the perpendicular orientation we see values from $5 \mathrm{~S} / \mathrm{m}$ to $13 \mathrm{~S} / \mathrm{m}$.

\section{D.2 Shielding Effectiveness}

Based on the measurements of conductivity, we can hypothesize that the SE of carbon fiber composite materials may not have the same values as those for metals. We will discuss the results from several papers [17-21] and attempt to understand how the SE of carbon-fiber composite aircraft should compare to that of metallic aircraft. In this technical note, we have presented the results in terms of penetration, which is just the reciprocal of SE. However, we will still use $\mathrm{SE}$ in this section so that when you refer back to the papers, a mental transition will not have to be made.

All measurements discussed in the following papers have to do with either single-layer or multilayer carbon-fiber composites in various orientational arrangements. In the layered composites, a combination of varying angles was used to simulate actual manufactured parts. Numerical modeling was used to verify results and to adjust parameters for evaluation. Most of the modeling is based on an effective-medium mixture theory which assumes that the resin and carbon fibers are present in fixed percentages of the total matrix. In the first paper, two woven carbon-fiber composites were measured and a theory developed to explain the SE. The main contribution to the dielectric constant is assumed to be from the conductivity. The dielectric constant is used to calculate the reflection and transmission coefficient, which in turn is used to calculate the SE of the layers,

$$
S E=-20 \cdot \log (|T|)
$$

The theoretical data for a plane wave incident on a sheet of carbon-fiber composite $[17,18]$ show that the SE increases exponentially at frequencies above $1 \mathrm{GHz}$. Measurements for X-band frequencies have shown that the $\mathrm{SE}$ of these carbon fiber composites is not much different from that of aluminum [17]. Theoretical data for a single layer show SE values of around $66 \mathrm{~dB}$ for frequencies from 1 to $18 \mathrm{GHz}$, and for a multilayer composite the lowest SE value was $86 \mathrm{~dB}$ [18]. In another paper [19], equivalent-layer models were developed for a single-ply carbon-fiber composite to explain the behavior of the reflection coefficient, transmission coefficient, and SE for a finite-element model. In this paper the SE was plotted for two different values of conductivity and for composites consisting of two panels or four panels. The data are summarized in Table D. 1 for frequencies less than $1 \mathrm{GHz}$. The data show that four panels provide better shielding than two panels and that carbon-fiber composites with higher conductivity also provide better shielding. 
Table D.1 Modeled conductivity for two-panel and four-panel composites.

(Frequencies less than 1GHz.)

\begin{tabular}{ccc}
\hline Conductivity $\boldsymbol{\sigma}(\mathbf{S} / \mathbf{m})$ & Two panels & Four panels \\
\hline $\mathbf{1 . 0}$ & 0.032 & 0.065 \\
$\mathbf{1} \cdot \mathbf{1 0}^{\mathbf{4}}$ & 32 & 39 \\
\hline
\end{tabular}

The final two papers $[20,21]$ show the dependency of SE on various parameters such as fiber orientation, conductivity, number of layers, and angle of incidence. For anisotropic laminated composites [20], it was shown that the SE is highly dependent on the polarization of the incoming electric field. For a laminate with a thickness of $4 \cdot 10^{-4} \mathrm{~m}$, the SE for an electric field polarized parallel to the anisotropy was $90 \mathrm{~dB}$, whereas, for the electric field polarized perpendicular to the anisotropy, the SE was $70 \mathrm{~dB}$ at a frequency of $1 \mathrm{MHz}$. The SE for a laminate containing four plies was about $70 \mathrm{~dB}$ at $1 \mathrm{GHz}$, as opposed to a laminate containing eight plies whose $\mathrm{SE}$ was about $100 \mathrm{~dB}$ at $1 \mathrm{GHz}$. For an eight-layer laminate whose panel orientation was $\left[0 \% 45^{\circ} / 90^{\circ}-45^{\circ}\right.$ ], the SE was approximately $70 \mathrm{~dB}$ at $1 \mathrm{MHz}$, compared to laminate whose panel orientation was $\left[0 \% 0^{\circ}\right]$ and had an SE of $80 \mathrm{~dB}$ at $1 \mathrm{MHz}$, and where the electric field was polarized perpendicular to the fiber orientation of the first panel. Finally, the last paper shows how various parameters change for a single-ply periodic composite material [21]. This paper also shows that as the conductivity increases from $10^{4} \mathrm{~S} / \mathrm{m}$ to $10^{7} \mathrm{~S} / \mathrm{m}$, the SE increases from $60 \mathrm{~dB}$ to $120 \mathrm{~dB}$ at $1 \mathrm{MHz}$. Above $10 \mathrm{GHz}$, the $\mathrm{SE}$ does shows no variability as a function of conductivity. For different fiber diameters, the SE can be changed by a factor of 1.5. As the angle of incidence increases, the SE increases. This model also shows that as the dielectric constant of the resin changes, the SE remains constant. And finally, for periodic structures, the reflection and transmission maxima and minima change directly as the ratio of thickness to wavelength.

These papers have shown us that the shielding effectiveness varies based on a whole array of parameters, and therefore it would be very difficult to determine whether the penetration for this particular aircraft was dependent on the carbon fiber structure. We also know that the seams in an aircraft built with carbon-fiber composites are not assembled in the same manner as for an allmetal aircraft, so additional leakage may come from the resulting apertures. We have observed that the penetration of this carbon-fiber composite aircraft is different from that for the Bombardier Global 5000 [1]. Based on the above discussion, we can conclude that some of this difference is due to the carbon-fiber composite structure, and some is due to different leakage apertures; but overall, the shielding values are comparable to those for a conventional metallic structure. 


\section{Appendix E. Penetration Data for HIRF Frequency Bands}

The peak and average field strengths in a HIRF environment are defined for specific frequency bands [2]. The frequency bands that correspond to our measured data are: (1) $200 \mathrm{MHz}$ to 400 $\mathrm{MHz}$, (2) $400 \mathrm{MHz}$ to $700 \mathrm{MHz}$, (3) $700 \mathrm{MHz}$ to $1000 \mathrm{MHz}$, (4) $1000 \mathrm{MHz}$ to $2000 \mathrm{MHz}$, (5) $2000 \mathrm{MHz}$ to $4000 \mathrm{MHz}$, (6) $4000 \mathrm{MHz}$ to $6000 \mathrm{MHz}$, (7) $6000 \mathrm{MHz}$ to $8000 \mathrm{MHz}$, (8) 8000 $\mathrm{MHz}$ to $12000 \mathrm{MHz}$, and (9) $12000 \mathrm{MHz}$ to $18000 \mathrm{MHz}$. If we determine the maximum penetration per compartment in these same frequency bands and plot it versus our measured data, we may find this to be more interesting for the HIRF community. Figures 137 to 139 are plots for the TEM and DRG antennas, in the vertical and horizontal polarization, at one angular position for each of the measured compartments. The black dotted line indicates the maximum penetration for that compartment for a DRG horn antenna in the horizontal polarization for all angular positions.

Figure 137 shows the penetration data as a function of the HIRF environment frequency bands for the receiving antenna in the main cabin and the transmitting antenna at an angular position of $0^{\circ}$. This is an angle where there is high electromagnetic penetration into the aircraft. We notice in several frequency bands that the TEM horn antenna in the horizontal polarization is above our maximum penetration line. By using these types of plots it is easy to pick out troublesome measurements. Penetration into the flight deck at an angle of $300^{\circ}$ is shown in Figure 138. Again the black dotted line shows the maximum penetration for all angles as a function of the frequency band. The TEM horn antenna shows more penetration in the lower frequency bands than does the DRG horn antenna in the horizontal polarization. Finally, Figure 139 shows the penetration for the receiving antenna in the rear cargo hatch, and the transmitting antenna at an angle of $240^{\circ}$. By looking through the individual frequency bands, we can isolate high penetration values for any antenna. If we use the maximum penetration for each compartment, we can then determine the peak and average field levels inside that compartment from the peak and average field levels outside of the aircraft. 

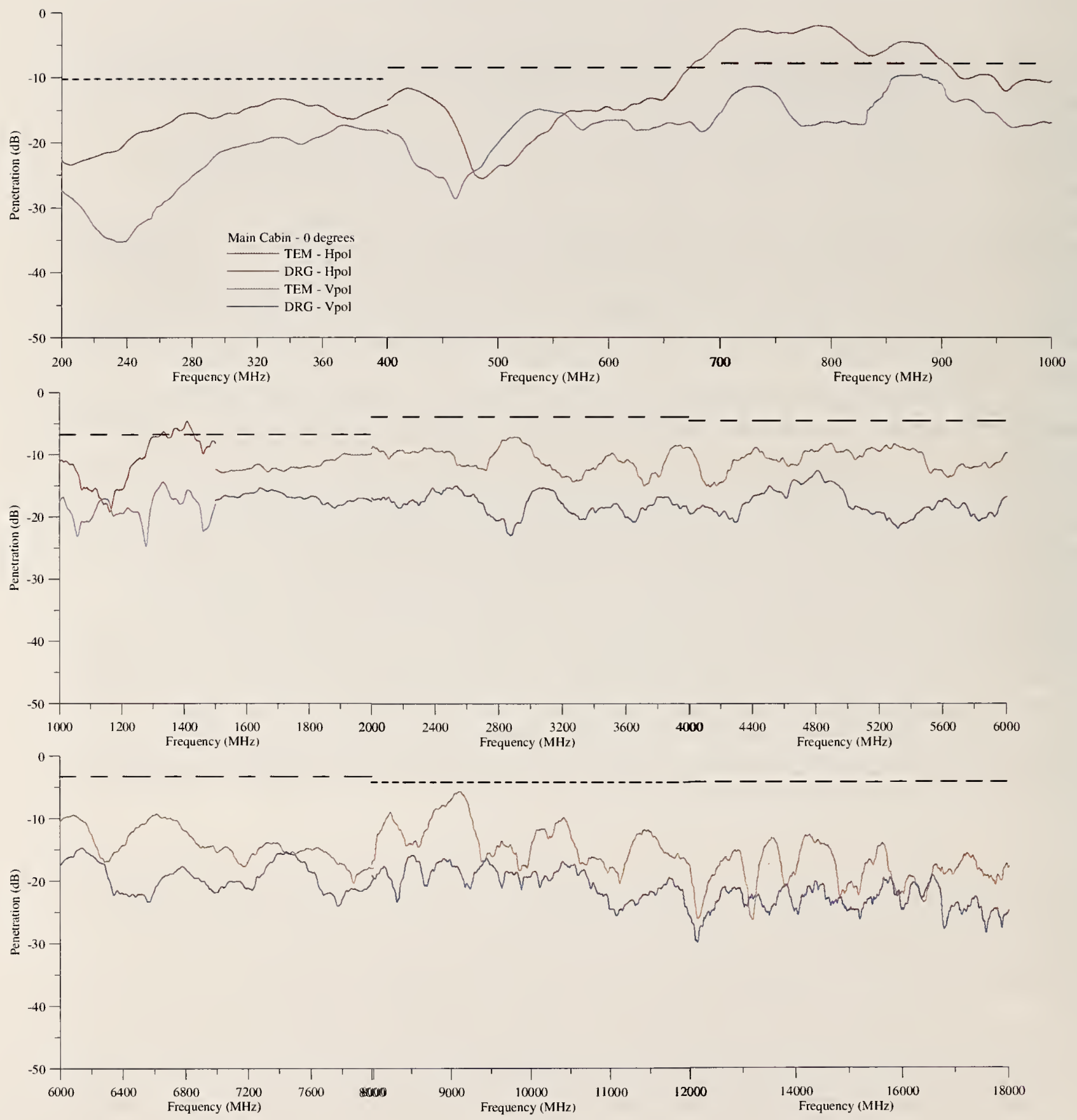

Figure 137. Frequency-band exploded view of the penetration for all antennas and polarizations for the transmitting tower placed at the $0^{\circ}$ position and the receiving antenna in the main cabin. 

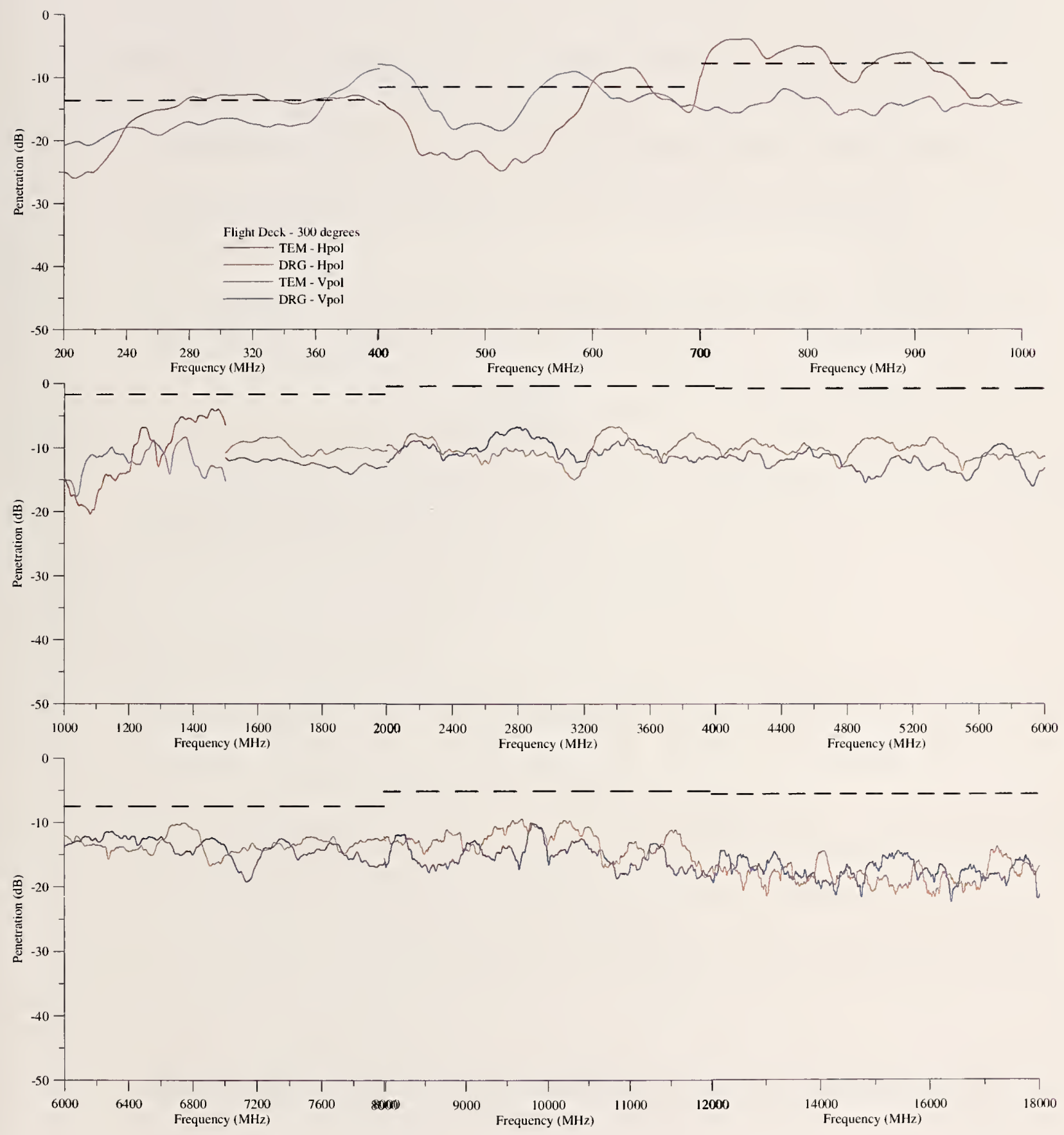

Figure 138. Frequency-band exploded view of the penetration for all antennas and polarizations for the transmitting tower placed at the $300^{\circ}$ position and the receiving antenna in the flight deck. 

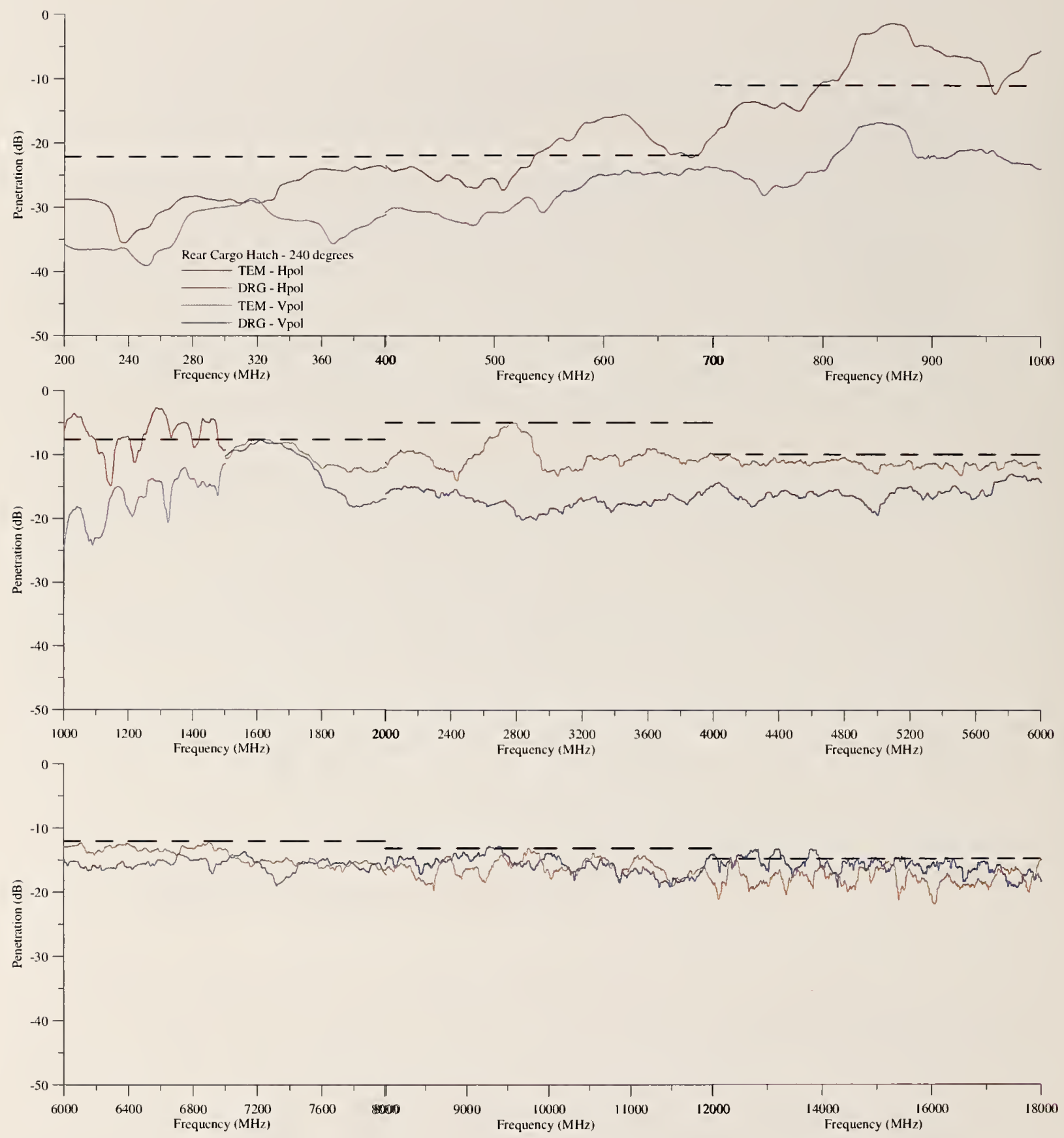

Figure 139. Frequency-band exploded view of the penetration for all antennas and polarizations for the transmitting tower placed at the $240^{\circ}$ position and the receiving antenna in the rear cargo hatch. 


\section{Appendix F. Beechcraft Premier IA Measurement Equipment List"}

The main equipment used in the evaluation of the Beechcraft Premier IA is listed below:

- Agilent N5230A PNA-L (300 kHz to $18 \mathrm{GHz}$ ) with a maximum of 16001 frequencies/trace.

- Megaphase Cables (4 $\mathrm{m}$ to $5 \mathrm{~m}$ )—we used phase stabilized cables to avoid phase drift with temperature variations.

- Miteq Optical Fiber Link MDDR/MDDT (11 GHz version) - this unit has the ruggedness and temperature characteristics for field use.

- Miteq Optical Fiber Link SCMR/SCMT (18 GHz version)-this unit has the ruggedness and temperature characteristics for field use.

- HP 11713A Switch Controller and HP 3781 Switches

- HP 59306A Relay Actuator

- HP E3615A DC Power Supply for relay control

- AML 0120L2403 Amplifier for DRG antennas

- Harrison 6205 DC Power Supply

- Two 1.2 m TEM Horn Antennas designed and built at the NIST Boulder Laboratories

- One $36 \mathrm{~cm}$ TEM Horn Antenna designed and built at the NIST Boulder Laboratories

- Three 3117 DRG antennas

- NIST-developed "RADAR" Labview program used to perform time/frequency transformations, time gating, and signal processing

- NIST-developed "multical" Labview program used to perform multiband VNA calibrations and to circumvent the 16001 frequency point limitation. This program permits an arbitrarily large number of frequency points. In practice we typically use as many as 48003 points.

\footnotetext{
* Certain commercial equipment, instruments, or materials are identified in this paper in order to adequately specify the experimental procedure. Such identification does not imply recommendation or endorsement by the National Institute of Standards and Technology, nor does it imply that the materials or equipment identified are necessarily the best available for the purpose.
} 



\section{NIST Technical Publications}

\section{Periodical}

Journal of Research of the National Institute of Standards and Technology-Reports NIST research and development in metrology and related fields of physical science, engineering, applied mathematics, statistics, biotechnology, and information technology. Papers cover a broad range of subjects, with major emphasis on measurement methodology and the basic technology underlying standardization. Also included from time to time are survey articles on topics closely related to the Institute's technical and scientific programs. Issued six times a year.

\section{Nonperiodicals}

Monographs-Major contributions to the technical literature on various subjects related to the Institute's scientific and technical activities.

Handbooks-Recommended codes of engineering and industrial practice (including safety codes) devel-oped in cooperation with interested industries, professional organizations, and regulatory bodies.

Special Publications-Include proceedings of conferences sponsored by NIST, NIST annual reports, and other special publications appropriate to this grouping such as wall charts, pocket cards, and bibliographies.

National Standard Reference Data Series-Provides quantitative data on the physical and chemical properties of materials, compiled from the world's literature and critically evaluated. Developed under a worldwide program coordinated by NIST under the authority of the National Standard Data Act (Public Law 90-396). NOTE: The Journal of Physical and Chemical Reference Data (JPCRD) is published bimonthly for NIST by the American Institute of Physics (AIP). Subscription orders and renewals are available from AIP, P.O. Box 503284, St. Louis, MO 63150-3284.

Building Science Series-Disseminates technical information developed at the Institute on building materials, components, systems, and whole structures. The series presents research results, test methods, and performance criteria related to the structural and environmental functions and the durability and safety characteristics of building elements and systems.

Technical Notes-Studies or reports which are complete in themselves but restrictive in their treatment of a subject. Analogous to monographs but not so comprehensive in scope or definitive in treatment of the subject area. Often serve as a vehicle for final reports of work performed at NIST under the sponsorship of other government agencies. Voluntary Product Standards-Developed under procedures published by the Department of Commerce in Part 10, Title 15, of the Code of Federal Regulations. The standards establish nationally recognized requirements for products, and provide all concerned interests with a basis for common understanding of the characteristics of the products. NIST administers this program in support of the efforts of private-sector standardizing organizations.

Order the following NIST publications-FIPS and NISTIRs-from the National Technical Information Service, Springfield, VA 22161.

Federal Information Processing Standards Publications (FIPS PUB)-Publications in this series collectively constitute the Federal Information Processing Standards Register. The Register serves as the official source of information in the Federal Government regarding standards issued by NIST pursuant to the Federal Property and Administrative Services Act of 1949 as amended, Public Law 89-306 (79 Stat. 1127), and as implemented by Executive Order 11717 (38 FR 12315, dated May 11,1973) and Part 6 of Title 15 CFR (Code of Federal Regulations).

NIST Interagency or Internal Reports (NISTIR)-The series includes interim or final reports on work performed by NIST for outside sponsors (both government and nongovernment). In general, initial distribution is handled by the sponsor; public distribution is handled by sales through the National Technical Information Service, Springfield, VA 22161, in hard copy, electronic media, or microfiche form. NISTIR's may also report results of NIST projects of transitory or limited interest, including those that will be published subsequently in more comprehensive form. 
U.S. Department of Commerce

National Bureau of Standards and Technology

325 Broadway

Boulder, CO 80305-3328

\section{Official Business}

Penalty for Private Use $\$ 300$ 